\title{
THE PHYSICS PROGRAM
}

\author{
OF A \\ HIGH-LUMINOSITY \\ ASYMMETRIC B FACTORY \\ AT SLAC
}

\begin{abstract}
A high-luminosity asymmetric energy $B$ Factory, proposed as an upgrade to the PEP storage ring at SLAC, provides the best opportunity to study CP violation as a means of testing the consistency of the Standard Model. If the phenomenon of CP violation is explained by the Standard Model simply through the non-zero angles and phase of the Kobayashi-Maskawa matrix, then there are precise relations between the $\mathrm{K}-\mathrm{M}$ parameters and the various measurable $\mathrm{CP}$-violating asymmetries in $B$ meson decay. Should these consistency relations fail, the origin of CP violation must lie outside the Standard Model framework. Our measurements would then lead to the first experiment-driven extensions of the Standard Model.

The $B$ Factory will also carry out a varied, high-quality program of studies of other aspects of the physics of $b$ quarks, as well as high-precision measurements in $\tau$ and charm physics. We describe a detailed scrics of mcasurements to be carricd out in the first few years at a peak luminosity of $3 \times 10^{33} \mathrm{~cm}^{-2} \mathrm{sec}^{-1}$, the initial luminosity goal of the $B$ Factory, as well as the program accessible to a larger data sample.
\end{abstract}

Work supported in part by the Department of Energy under contracts DE-AC03-76SF00515, DE-AC03-76SF00098 and DE-AC03-81-ER40050

Printed in the United States of America. Available from the National Technical Information Service, U.S. Department of Commerce, 5285 Port Royal Road, Springfield, Virginia 22161. Price: Printed Copy A12, Microfiche A01. 


\section{FOREWORD}

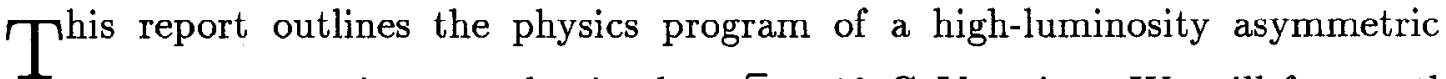
energy storage ring complex in the $\sqrt{s}=10 \mathrm{GeV}$ region. We will frequently refer to these storage rings as a " $B$ Factory", as their primary goal is the production of a sufficient number of $B \bar{B}$ meson pairs in the appropriate configuration to allow the first measurement of $\mathrm{CP}$ violation in the $B$ meson system. The appropriate configuration, as we will demonstrate, is that the $B \bar{B}$ pairs must be produced in the decay of the $\Upsilon(4 S)$ resonance, with the $\Upsilon(4 S)$ system moving in the laboratory. Our studies have shown that the optimal energy asymmetry for this purpose lies in the region of $9 \mathrm{GeV}$ for the electron beam and $3 \mathrm{GeV}$ for the positron beam. We will use these values in most instances, although for certain studies, such as those of $B_{s}$ mixing, a somewhat higher asymmetry is desirable. These storage rings have been designed to be contained in the existing tunnel of the PEP storage ring, which permits considerable savings in cost and time. The details of the accelerator design are contained in the companion report A Feasibility Study for an Asymmetric B Factory Based on PEP.

It must be strongly emphasized, however, that the capabilities of the program extend far beyond the primary motivation. While the goal of stringent tests of the consistency of the Standard Model is quite sufficient motivation on its own for this project, a rich program of experiments in a large number of other areas is a natural adjunct to the search for $\mathrm{CP}$ violation. Studies of rare $B$ decays to unprecedented sensitivity are possible, as are measurements of particular Kobayashi-Maskawa matrix elements, measurements of $B$ meson hadronic branching ratios and measurements of transitions within the $\Upsilon$ system. Along with the production of large numbers of $B$ mesons in the $\sqrt{s}=10 \mathrm{GeV}$ region we would produce a similar number of $\tau \bar{\tau}$ pairs in a particularly favorable region for high-precision $\tau$ studies. Charmed meson and baryon production is also substantial. As the charmed particles are produced with distinguishable decay vertices, this leads to exciting prospects for very high sensistivity studies of $D^{\circ} \bar{D}^{\circ}$ mixing, rare $D$ decays and 
excited charmed mesons and baryons.

Our report contains a variety of examples drawn from each of these areas of interest. We consider scenarios involving the accumulation of conservative amount $\left(30 \mathrm{fb}^{-1}\right)$ of integrated luminosity at the outset, as well as the capability of a program which accumulates a total luminosity of $300 \mathrm{fb}^{-1}$. This document is the outcome of studies carried out over the summer of 1989 in a series of meetings at SLAC and Caltech, which have seen the enthusiastic participation of a substantial number of physicists from a variety of institutions:

- U.C. Intercampus Institute for Research at Particle Accelerators
$\diamond$ Alan Eisner

- U.C. Irvine

$\diamond$ Mark Mandelkern

- U.C. Santa Barbara

$\diamond$ Rollin Morrison, Michael Witherell

- U.C. Santa Cruz

$\diamond$ Patricia Burchat, Joel Kent

- U.C. San Diego

$\diamond$ Robin Erbacher, Wayne Vernon

- California Institute of Technology

$\diamond$ Gerald Eigen, David Hitlin, Frank Porter, Alan Weinstein, William Wisniewski

- University of Colorado

$\diamond$ Steven Wagner

- Columbia University

$\diamond$ Paolo Franzini, Michael Tuts

- University of Indiana

$\diamond$ Doris Averill, Arthur Snyder

- Lawrence Berkeley Laboratory

$\diamond$ Gerson Goldhaber, Piermaria Oddone, Natalie Roe, Michael Ronan, Martin Spahn 
- McGill University

$\diamond$ David MacFarlane

- Stanford Linear Accelerator Center

$\diamond$ John Bartelt, Elliott Bloom, Fatin Bulos, Dieter Cords, Claudio Dib, Jonathan Dorfan, Isard Dunietz, Frederick Gilman, Gary Godfrey, Thomas IIyer, Garth Jensen, David Leith, Helmut Marsiske, Yosef Nir

- SUNY Stony Brook

$\diamond$ Juliet Lee-Franzini

The Study was divided into Working Groups. The Working Group leaders were:

- CP Violation - John Bartelt

- Other $B$ Physics - Natalie Roe and Arthur Snyder

- $\Upsilon$ Physics - Michael Tuts

- $\tau$ Studies - Helmut Marsiske

- Charm Studies - Michael Witherell

- Detector Simulation - Alan Weinstein

The Study was coordinated by David Hitlin, who served as editor of this report. 


\section{TABLE OF CONTENTS}

FOREWORD ................... . i

1. INTRODUCTION . . . . . . . . . . . . . . . . . . . . 1

1.1 The Role of a $B$ Factory in the SlaC Program . . . 5

2. STANDARD MODEL PREDICTIONS FOR CP VIOLATION

IN $B^{\circ}$ MESON DECAY . . . . . . . . . . . . . . . 8

2.1 The Unttarity Triangle . . . . . . . . . . . . . . . 9

2.2 CP Violation with Neutral $B$ Mesons . . . . . . 10

2.3 Constraining the Unitarity Triangle . . . . . . . 13

2.4 Ranges of CP Asymmetries for $B^{o}$ Mesons . . . . 21

2.5 Luminosity Considerations . . . . . . . . . . . . 24

2.6 Asymmetric Machine Operating at the $\Upsilon(4 S)$. . . 27

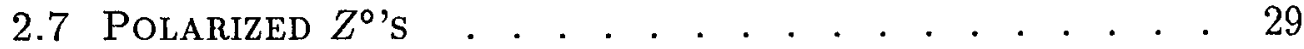

3. MONTE CARLO AND DETECTOR SIMULATION . . . . 36

3.1 Monte Carlo Event Generation . . . . . . . . 37

3.2 Detector Simulation . . . . . . . . . . . . 38

3.3 Tracking System . . . . . . . . . . . . . . . . 40

3.4 Electromagnetic Calorimetry . . . . . . . . . . 44

3.5 PARTICLE IDENTIFICATION . . . . . . . . . . . . . 46

3.6 Vertexing . . . . . . . . . . . . . . . . . . . 49

3.7 Analysis ToOls . . . . . . . . . . . . . . . . . 49

4. MASKING, BEAMPIPE AND VERTEXING_ISSUES . . . . 50

4.1 MASKING . . . . . . . . . . . . . . . . . . . 50

4.2 Beampipe Cooling . . . . . . . . . . . . . . . . 51

4.3 Ventex Issues . . . . . . . . . . . . . . . . . . 51 
5. MEASURING CP VIOLATION AT AN ASYMMETRIC

COLLIDER . . . . . . . . . . . . . . . . 57

5.1 Introduction . . . . . . . . . . . . . . . . . 57

5.2 Using CP Eigenstates at the $\Upsilon(4 \mathrm{~S})$ to Measure

CP Violation . . . . . . . . . . . . . . . . 60

5.3 Physics and Detector Simulation . . . . . . . . 65

5.4 Measuring $\sin 2 \beta$ with $B \rightarrow J / \psi K_{s}^{\circ}$. . . . . . . . . 69

5.5 Measuring $\sin 2 \alpha$ with $B \rightarrow \pi^{+} \pi^{-}$. . . . . . . . . 83

5.6 Measuring $\sin 2 \gamma$ With $B_{s} \rightarrow \rho K_{s}^{o}$. . . . . . . . . . 84

5.7 Comparing Different Boosts and Beampipes . . . . 91

5.8 Comparing Symmetric and Asymmetric $B$ Factories 94

6. $B$ DECAY PHYSICS OTHER THAN CP VIOLATION · . 100

6.1 Introduction . . . . . . . . . . . . . . . 100

$6.2 B_{s} \bar{B}_{s}$ MiXING . . . . . . . . . . . . . . . . . . 101

$6.3 b \rightarrow c$ Hadronic Decays-Understanding the Weak

DeCAY MEChanisM . . . . . . . . . . . . . . 117

$6.4 b \rightarrow c$ Semi-Leptonic DeCays-Tagging $B$ Mesons by

Partial Reconstruction . . . . . . . . . . . . . 123

$6.5 b \rightarrow u$ DECAYS-Determination of $V_{u b}$. . . . . . . . 124

$6.6 b \rightarrow s \gamma$ : Penguin Diagrams . . . . . . . . . . . . 150

$6.7 B^{+} \rightarrow \tau \nu_{\tau}$ : Determination of $f_{B}$. . . . . . . . . 156

7. $\Upsilon$ PHYSICS AT AN ASYMMETRIC $B$ FACTORY . . . . . 162

7.1 Introduction . . . . . . . . . . . . . . . . . . 162

7.2 A Review of the Present Status . . . . . . . . . 164

$7.3 \Upsilon$ Physics Capabilities at High Luminosities . . . 169

7.4 Detector Constraints . . . . . . . . . . . . 186

7.5 Conclusions . . . . . . . . . . . . . . . . . 193 
8. TAU PHYSICS AT A $B$ FACTORY . . . . . . . . . . . . 197

8.1 Introduction . . . . . . . . . . . . . . . . . . 197

8.2 Limits on the Tau Neutrino mass . . . . . . . . 198

8.3 Measurement of the Cabibbo Angle . . . . . . . 202

8.4 Second-Class Currents . . . . . . . . . . . . . 203

8.5 RARE Dechys . . . . . . . . . . . . . . . . . . 206

8.6 Structure of the $\tau-W-\nu_{\tau}$ Vertex . . . . . . . 208

8.7 Measurement of $B_{e} / B_{\mu}$. . . . . . . . . . . . . . . 209

8.8 Measurement of the Tau Lifetime . . . . . . . . 211

8.9 Tau Branching Fractions . . . . . . . . . . . . 214

9. CHARM PHYSICS . . . . . . . . . . . . . . . 218

9.1 Introduction . . . . . . . . . . . . . . . . . . 218

$9.2 D^{o}-\overline{D^{o}}$ MiXING . . . . . . . . . . . . . . . . . 220

9.3 CP Violation . . . . . . . . . . . . . . . . . . 228

9.4 Semi-leptonic Decays of Charm . . . . . . . . . 229

9.5 Meson Spectroscopy . . . . . . . . . . . . . . 231

9.6 Baryon Spectroscopy and Decays . . . . . . . 237

9.7 Summary of Charm Physics Capabilities . . . . . . 239

10. SUMMARY AND CONCLUSIONS . . . . . . . . . . . 242 APPENDIX ................... . 244 


\section{INTRODUCTION}

$\mathrm{T}$ ests of the consistency of the Standard Model through high-precision measurements of the Kobayashi-Maskawa matrix elements provide some of the most exciting experimental prospects for the 1990 's. The centerpiece of this type of experiment is the study of CP violation in the $b$ quark system. The measurement of $\mathrm{CP}$-violating parameters requires a large sample of $B^{o} \bar{B}^{o}$ meson pairs, produced in a novel configuration: in $\Upsilon(4 S)$ decay, where the $\Upsilon(4 S)$ is moving in the laboratory. The construction of an asymmetric energy $e^{+} e^{-}$ storage ring in the $\sqrt{s}=10-11 \mathrm{GeV}$ region is thus the key to an extraordinarily interesting program of detailed consistency tests of the Standard Model, making possible experiments which are complementary, as well as supplementary, to tests envisioned at the SSC, and which can be performed in the coming decade, before the SSC program commences. This report will discuss the physics motivation for the construction of a high-luminosity asymmetric $B$ Factory at SLAC, and will provide illustrative examples of experimental capabilities in the areas of $B$ physics, $\Upsilon$ physics, $\tau$ physics and charm physics. The physics program we envision deals with fundamental issues within the Standard Model. Important results will be accessible at the outset, with integrated luminosity well below the eventual goal. It represents a long-term program with unique capability, which will result in the first observation of $\mathrm{CP}$ violation in $B$ meson decay, allowing, for the first time, detailed consistency tests of the Standard Model. With sufficient integrated luminosity, it will also permit an exploration of the mechanism of CP violation.

The accelerator required to carry out this program, described in the accompanying document, A Feasibility Study for an Asymmetric $B$ Factory Based on PEP, is a novel one, in that the $e^{+}$and $e^{-}$beams are of unequal energy. The asymmetric energies, which are absolutely required in order to carry out the core program of $\mathrm{CP}$ violation measurements, also result in a substantial reduction of combinatoric background in more conventional experiments. This is valuable, in particular, in producing large, very clean tag samples, for the investigation of rare processes and semileptonic decays. The

Measurements of $C P$-violating asymmetries in $B$ meson decay, crucial tests of the Standard Model, are best done at an asymmetric storage ring operating at the $\Upsilon(4 \mathrm{~S})$ resonance 
We discuss the physics capabilities of the program at two levels of integrated luminosity

The initial data sample should suffice to discover $C P$ violation in $B$ meson decay. The largcr sample will allow a detailed exploration of the phenomenon peak luminosity target of the upgrade is $3 \times 10^{33} \mathrm{~cm}^{-2} \mathrm{sec}^{-1}$. We would expect the physics program to commence as the storage ring luminosity comes up at a luminosity somewhere above $10^{33} \mathrm{~cm}^{-2} \mathrm{sec}^{-1}$ and rapidly evolves to the regime around $3 \times 10^{33} \mathrm{~cm}^{-2} \mathrm{sec}^{-1}$. Two years after the physics run begins, we expect to have accumulated an integrated luminosity of $30 \mathrm{fb}^{-1}$. We have used this value to develop an initial physics program, which in addition to making the first measurement of $C P$ violation in the $B$ meson system, will produce a host of exciting results in several areas. We have also extended our studies to a data sample of $300 \mathrm{fb}^{-1}$, which we regard as the ultimate goal of the program. We would expect, to devote sufficient time to accelerator $R \& D$ to make it plausible to expect an increase in peak luminosity to the region of $10^{34}$ over time. It should be noted that the acquisition of a $300 \mathrm{fb}^{-1}$ sample is possible without a luminosity this high; a $10^{34}$ machine can do it in three years. Our unit of time is a "Snowmass Year" (=10 $10^{7}$ seconds), which is calculated to take into account the difference between peak and average luminosity as well as accelerator and detector down-time.

The plan of this Report is as follows: we will first investigate the present and projected future status of the determination of the "unitarity triangle" which summarizes the tests of consistency of the Standard Model. Next, the Monte Carlo tools used in the studies will be described, as will the parameters for the model detector employed. Subsequent chapters will then present the results of studies of experimental capabilities in $B$ meson decay (CP violation; $B_{s}$ mixing; and "conventional" $B$ physics, such as searches for rare decays and measurements of $\left.\left|V_{u b}\right|\right)$, studies of the $\Upsilon$ system in unprecedented detail, charmed hadrons and $\tau$ decay.

The 30 and $300 \mathrm{fb}^{-1}$ samples correspond to $2.5 \times 10^{7}$ and $2.5 \times 10^{8}$ produced $\bar{B} B$ pairs, respectively. At an asymmetric machine the initial sample should suffice to observe the phenomenon of CP violation in the decay to the CP eigenstate $B^{o}, \bar{B}^{o} \rightarrow J / \psi K_{s}^{o}$, while the full sample will allow a detailed exploration of the phenomenon in a variety of decay modes. These measurements are discussed in Chapter 5. The large number of $B$ mesons which will be reconstructed in conventional decay modes are interesting in their own right, but also 
provide a very large sample of $B$ "tags" which makes possible the measurement of, for example, $\left|V_{u b}\right|$ via the exclusive decay mode $B \rightarrow \pi l \nu$ and $B \rightarrow \rho l \nu$ and allows a sensitive search for the decay $B \rightarrow \tau \nu_{\tau}$ and thus a measurement of $f_{B}$, the $B$ meson pseudoscalar coupling constant. These topics are covered in Chapter 6.

$B$ meson studies are not the only interesting physics accessible to a high luminosity asymmetric storage ring. A properly-designed detector will allow measurements in the $\Upsilon$ system of unprecedented detail, as discussed in Chapter 7. A sample of $\tau$ pairs of the same size as that of the $B$ sample will also be obtained, as the $\tau \tilde{\tau}$ cross section is equal to the $\Upsilon(4 S)$ cross section. Detailed studies (see Chapter 8 ) have confirmed that $\tau$ experiments of great interest can be carried out with high precision. Charm studies can also be brought to a new level. Chapter 9 considers in detail a number of important questions in the charm sector, including the search for $D^{\circ} \bar{D}^{o}$ mixing, which can be addressed in a particularly clear way. It is worth noting that the study of excited charmed meson and baryons, still in its infancy, has been advanced to its present state largely through experiments at the $\Upsilon(4 \mathrm{~S})$. Such experiments are, of course, not accessible in $\psi(3770)$ data, and are far from optimally carried out in the $4-5 \mathrm{GeV}$ region.

While the most recent lower limits on the $t$ quark mass would appear to place $t$ quark scarches in the domain of the Tevatron and the SSC, those measurements requiring large $B$ meson and $\tau$ samples would appear to be most practical at high-luminosity $e^{+} e^{-}$storage rings. In the case of the $\tau$, this is perhaps obvious, as the $\tau$ has to date been observed only in $e^{+} e^{-}$annihilation, and large, background-free samples of $\tau$ 's are likely, for the foreseeable future, to be obtainable only by this technique. While $B$ mesons are copiously produced in high-energy hadronic collisions, only a handful have been observed to date, and the prospects for obtaining a very large tagged sample of $B$ mesons at a hadron machine appear daunting. As of this writing, a total of $\sim 10^{6} B$ mesons have been produced in decays of the $\Upsilon(4 S)$, while only a few hundred hadronic decays have been reconstructed. An increase in the produced sample of two orders of magnitude, with a Lorentz-boosted $\Upsilon(4 S)$ and a high-quality detector, promises
$A$ variety of topics, drawn from the areas of $\Upsilon$, charm and $\tau$ physics, are discussed in addition to the $B$ decay physics program
In the $\sqrt{s}=10 \mathrm{GeV}$ region, the numbers of $B$ mesons, charmed particles and $\tau$ 's produced are roughly comparable 
Even with modest initial luminosity goals, we will overtake the CESR data sample in a short time

Running on the $\Upsilon(4 S)$ in an asymmetric configuration produces signal-to-background ratios which are superior to both $\Upsilon(4 S)$ running in the symmetric case, due to the ability to reconstruct individual $B$-decay vertices, and to running above the $\Upsilon(1 S)$, due to the larger fraction of the cross section which represents $B \bar{B}$ production to yield the first measurements of $\mathrm{CP}$ violation in $B$ meson decay.

Given sufficient priority, the construction of new, highluminosity storage rings in the PEP tunnel and a new high quality detector can be completed in about five years. Building a high-luminosity asymmetric $B$ factory at SLAC results in a considerable savings of money and time. The use of the PEP tunnel and many PEP accelerator components could save as much as $11 / 2$ years, as it will not be necessary to retain an Architectural and Engineering firm, to file Environmental Impact statements or, indeed, to do any substantial amount of civil construction.

It is important, nonetheless, to ask what could be accomplished in the intervening time by facilities which are currently in operation. The machine with the highest luminosity in the $\Upsilon$ region is CESR, which has achieved a peak luminosity of $1 \times 10^{32} \mathrm{~cm}^{-2} \mathrm{sec}^{-1}$, and has detailed plans to improve the luminosity over the next several years. It is, of course, very difficult to predict the course of such an improvement program. For illustrative purposes, we have made the assumption that the development of CESR luminosity with time will show a linear rise to $5 \times 10^{32}$ over five years. As about $1 / 3$ of CESR data is taken below the $\Upsilon(4 \mathrm{~S})$ to allow study of continuum background, this leads to an integrated luminosity at the $\Upsilon(4 S)$ of $9.2 \mathrm{fb}^{-1}$ over five years, representing an order of magnitude increase over existing $B$ meson samples. It is worth noting that the lower backgrounds to $B$ meson reconstruction at an asymmetric machine require less off-resonance running; fifteen percent should suffice.

The first few years of running of the proposed asymmetric machine therefore nearly triple the total world sample, even with the initial luminosity assumptions. The ultimate sample represents a factor of thirty increase over the sample projected to exist at turn-on. This increase is important in itself, but the fact that the $B$ mesons will be produced in motion at the $\Upsilon(4 S)$ represents a new and most important feature. As the separate decay vertices of $B$ and $D$ mesons are distinguishable, the combinatoric background under exclusive-mode peaks will be substantially reduced, pro- 
viding clean, high-statistics tag samples. This is investigated in some detail in Chapter 6. More importantly, the ability to measure the decay time between vertices in the decay of $B^{o}$ and $\bar{B}^{\circ}$ to CP eigenstates makes the search for CP violation viable. The energy asymmetry provides unique capability to study $\mathrm{CP}$ violation. The measurement of $\mathrm{CP}$-violating asymmetries in decays to $\mathrm{CP}$ eigenstates is not possible at a symmetric machine, as the $\mathrm{CP}$ asymmetry integrates to zero. While other CP-violating effects can conceivably be measured at a symmetric machine, only decays to $\mathrm{CP}$ eigenstates can be rigorously, that is without uncertainties due to hadronic physics, related to the fundamental parameters of the Kobayashi-Maskawa matrix. Such a measurement is not possible with a large sample of $B$ mesons produced by stationary $\Upsilon(4 \mathrm{~S})$ decays. The method proposed herein, discussed in Chapter 5, has been stated to enjoy a factor of 4-5 advantage in required luminosity over a technique based on producing $B$ mesons above the $\Upsilon(4 S)$. This assessment did not take into account the debilitating effect of background. The asymmetric machine has additional substantial advantages in this regard. Reduction of combinatoric background by reconstruction of distinct vertices is one advantage. A second is the ability to take CP-relevant data on the $\Upsilon(4 S)$, where the signal-to-background ratio for $B$ reconstruction is far superior to that obtainable either in the resonance region above it or in the continuum. The effect of background on the measurement of CP asymmetries is discussed in Chapter 5 .

\subsection{The Role of a B Factory in the SLAC Physics} PROGRAM

In recent months, SLAC has been pondering new directions for its current program, seeking directions which are consistent both with its long-term goals and with the needs of a national program. It has been decided that SLAC will not serve as a major participant in the construction of an SSC detector but rather will maintain its role as the mainstay of the electron-positron arm of the national program. This provides crucial diversity to the national program, as a
The oft-quoted factor of 4 to 5 advantage of the asymmetric $\Upsilon(4)$ configuration over the symmetric case above the $\Upsilon(4)$ is an underestimate, as it does not account for the additional advantage which accrues to a superior rejection of background, with its concomitant improvement in signal-to-noise ratio

The $B$ factory will allow SLAC to maintain its leadership in the $e^{+} e^{-}$arm of the national program through the coming decade 
The Hilton Head HEPAP Subpanel identified a $B$ factory as an important facility for the '1990's

Energies of 9 and $3 \mathrm{GeV}$ are optimal for measuring $C P$ violating asymmetries strong, forward-looking electron-positron program is an important adjunct to the proton-based program. This proposed role for SLAC has been endorsed by HEPAP and the DOE; there is general agreement that this is a fruitful role for SLAC in the context of the future U.S. elementary particle physics program.

SLAC is at the forefront of the worldwide $R \& D$ effort on a high energy $(\geq 400 \mathrm{GeV})$ linear collider; this direction must be vigorously pursued. The amount of $R \& D$ required and the cost of a large new linear collider preclude the start of construction of such a machine before SSC construction costs begin to taper off (1997 at the earliest). This provides a natural window for a high-quality project at SLAC which meets the proper criteria of physics interest, cost and time-scale, and which complements ongoing SLC-based efforts. Only with such a project will SLAC retain its high-quality faculty and staff and preserve a vital university uscr community. Many people in the experimental community are seeking accelerator-based opportunities which are complementary to the SSC program. This was clearly evident in the report of the 'Hilton Head' HEPAP subpanel, which prominently identified a high luminosity $B$ factory as an important facility for the 1990's. The high luminosity asymmetric storage ring facility in the $\Upsilon$ region provides an exceptionally rich physics program encompassing bottom, charm and $\tau$ physics which will serve as a) the bridge between the current SLAC program and the linear collider era and b) a very rich and diverse physics program which is of great interest to the national and international community.

The physics potential of a machine of this type is widely recognized: KEK, DESY, Novosibirsk, PSI, SLAC and Cornell have been exploring the feasibility of such a machine. SLAC has many advantages which make it a natural place to build a $B$ factory. The facility we are proposing makes use of the existing PEP tunnel, interaction region halls and utilities, as well as many machine components. Both rings, one at $9 \mathrm{GeV}$ and the othèr at $3 \mathrm{GeV}$, optimal energies for the measurement of CP-violating asymmetries, would be constructed in the existing PEP tunnel. The vacuum and cooling systems are designed to support currents which would allow 
a peak luminosity of $10^{34} \mathrm{~cm}^{-2} \mathrm{sec}^{-1}$, although the initial luminosity goal is $3 \times 10^{33}$. Basing the project on the PEP complex results in a considerable savings of money and time and takes advantage of the existing PEP infra-structure. The project as a whole benefits from the SLAC's managerial and technical expertise, its long experience in electron-positron storage rings and its existing relationship with the DOE. The enthusiastic participation of Lawrence Berkeley Laboratory and major university groups in the design and construction of the accelerator promises to bring a considerable amount of additional accelerator expertise, as well as engineering and technical manpower, to bear on the project.

The physics program described in this Report addresses important questions, provides interesting technical challenges both in detector and accelerator design and motivates a project of the quality and scope which SLAC must have to maintain its forefront position. The main goals, the first measurement of CP violation in $B$ decay, and the detailed exploration of the phenomenon, are of prime importance. A highluminosity asymmetric $B$ Factory should be SLAC's next major endeavor.
The $B$ factory project would be actively supported by $L B L$ and a variety of major university user groups 


\section{Standard Model Predictions for CP Violation in $B^{0}$ Meson Decay}

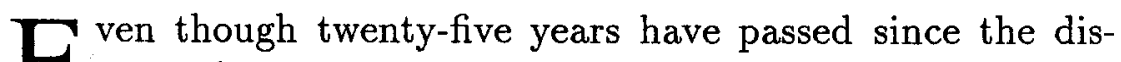
$E_{\text {covery }}{ }^{1}$ of $\mathrm{CP}$ violation, its observation only within the $K$ meson system has left us with different hypotheses as to its origin. However, during that time the Standard Model of the electroweak interactions has been developed, in which $\mathrm{CP}$ violation has a natural place, the Cabibbo-KobayashiMaskawa (CKM) mixing matrix:

$$
V=\left(\begin{array}{lll}
V_{u d} & V_{u s} & V_{u b} \\
V_{c d} & V_{c s} & V_{c b} \\
V_{t d} & V_{t s} & V_{t b}
\end{array}\right) .
$$

A unique CP-violating phase could occur with three generations of quarks. Independent of any phase convention in defining the matrix, the phase could be taken to be

$$
\arg \left(V_{u s} V_{u b}^{*} V_{c s}^{*} V_{c b}\right)
$$

In the Standard Model explanation for $C P$ violation, large $C P$-violating asymmetries are predicted in neutral $B$ decays.
The question before us is whether this is indeed the origin of $\mathrm{CP}$ violation as it is observed in nature. If this phase were to explain what is observed in $K$ decays, then large CP-violating asymmetries would be predicted in neutral $B$ meson decays.

This chapter presents the current status of what can be said about such asymmetries in the context of our knowledge of the experimental constraints on the parameters of the Standard Model, updating and extending previous work. ${ }^{2-5}$ We use the unitarity triangle of the CKM matrix to show these constraints as a function of top quark mass. The CPviolating asymmetries for neutral $B$ meson decays in which we are interested are related to the angles of the unitarity triangle. The consequent range of asymmetries allowed for a given type of $B$ decay is evaluated, and the luminosity of 
an electron-positron collider needed in order to guarantee a statistically significant measurement of $C P$ violation in one or more types of $B$ decay is then presented.

\subsection{The Unitarity TRiangle}

Unitarity of the $3 \times 3$ CKM matrix yields

$$
V_{u d} V_{u b}^{*}+V_{c d} V_{c b}^{*}+V_{t d} V_{t b}^{*}=0 .
$$

The unitarity triangle is just a geometrical presentation of this equation in the complex plane. We can always choose to orient the triangle so that $V_{c d} V_{c b}^{*}$ lies along the horizontal axis. This is equivalent to choosing a phase convention. In any case, the parametrization adopted by the Particle Data Group ${ }^{7}$ makes $V_{c b}$ real and $V_{c d}$ real to a very good approximation. Also, $V_{u d} \approx 1, V_{t b} \approx 1$, and $V_{c d} \approx-\sin \theta_{C}=-0.22$, and Eq. (2.3) now becomes

$$
V_{u b}^{*}+V_{t d}=\left|V_{c d} V_{c b}\right|
$$

which is shown as the unitarity triangle in Figure 2.1.

$\mathrm{CP}$-violating asymmetries between $B^{0}$ and $\bar{B}^{0}$ mesons decaying to CP eigenstates are proportional to $\sin (2 \phi)$, where $\phi$ stands for one of the angles (labelled $\alpha, \beta$, and $\gamma$ in Figure $2.1)$ of the triangle. Rescaling the triangle by $\left[1 /\left(\left|V_{c d} V_{c b}\right|\right)\right]$, the coordinates of the three vertices $A, B$, and $C$ become:

$$
A\left(\frac{\operatorname{Re} V_{u b}}{\left|V_{c d} V_{c b}\right|},-\frac{\operatorname{Im} V_{u b}}{\left|V_{c d} V_{c b}\right|}\right) ; \quad B(1,0) ; \quad C(0,0)
$$


Figure 2.1 Representation in the complex plane of the triangle formed (a) by the CKM matrix elements $V_{u b}^{*}, V_{c d}$. $V_{c b}^{*}$, and $V_{t d}$, and the rescaled triangle (b) with vertices at $A(\rho, \eta), B(1,0)$, and $C(0,0)$. $A$ relevant $B^{0}$ decay mode is indicated for the angle involved in the corresponding $C P$-violating asymmetry.

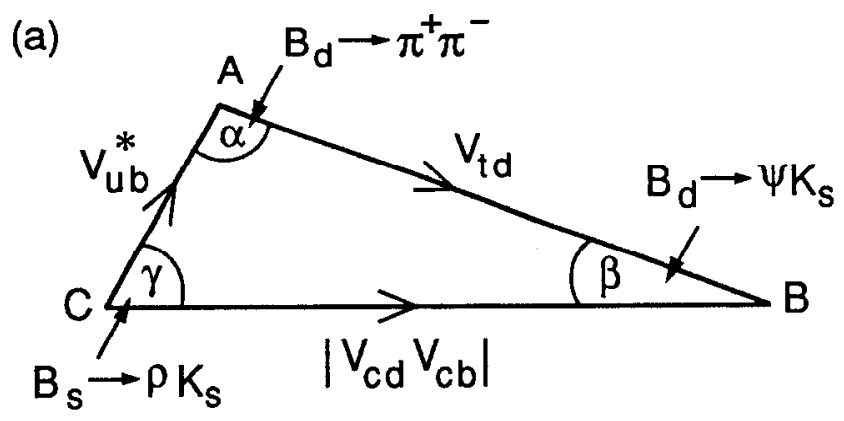

(b)

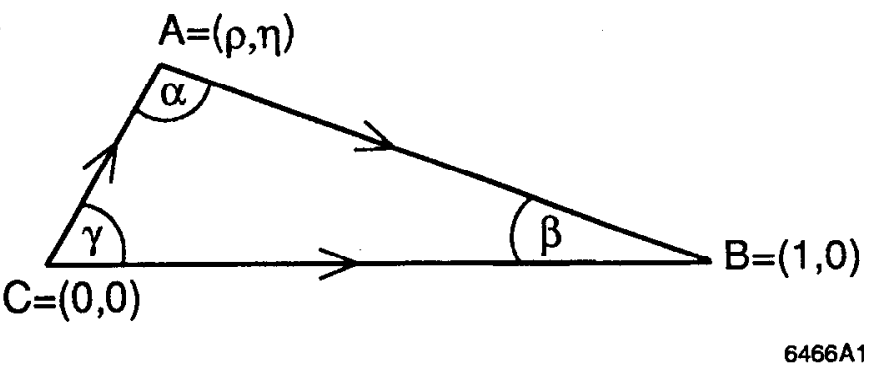

In the Wolfenstein parametrization, ${ }^{9}$ which is just the small mixing-angle approximation given here with the matrix elements expressed in terms of powers of $\sin \theta_{C}$, the coordinates of the vertex $A$ are $(\rho, \eta)$. What remains for Section 4 is to constrain the point $A$ by using the experimental data which are presently available.

\subsection{CP Violation with Neutral $B$ Mesons}

The decay rate of a time-evolved, initially pure $B^{0}\left(\bar{B}^{0}\right)$ into a CP-eigenstate, $f$, is: ${ }^{10}$

$$
\begin{aligned}
& \Gamma\left(B_{\text {phys }}^{0}(t) \rightarrow f\right) \propto e^{-\Gamma t}[1-\operatorname{Im} \lambda \sin (\Delta m t)] \\
& \Gamma\left(\bar{B}_{\text {phys }}^{0}(t) \rightarrow f\right) \propto e^{-\Gamma t}[1+\operatorname{Im} \lambda \sin (\Delta m t)] .
\end{aligned}
$$

CP-violating effects are manifest through the presence of the interference term $\operatorname{Im} \lambda$. For the processes under consideration here, the CP violation arises from the quantum mechanical 
interference of amplitudes corresponding to two paths to the same final state, one of which involves $B^{0}-\bar{B}^{0}$ Inixing. Possible small $\mathrm{CP}$-violating effects in the decay amplitude itself are neglected. Care must be taken whether the final state is CP-even or odd, since that flips the sign of the interference term: ${ }^{11} \operatorname{Im} \lambda_{\text {odd }}=-\operatorname{Im} \lambda_{\text {even }}$. We always quote the interference terms obtained for $\mathrm{CP}$-even eigenstates.

For a given quark subprocess, Table 2.1 lists a few corresponding hadronic final states and the relevant interference term, $\operatorname{Im} \lambda$, responsible for CP-violation (stated in terms of the angles in the unitarity triangle).

Table 2.1

Decay Modes and Interference Terms for Various Classes

\begin{tabular}{|c|c|c|}
\hline $\begin{array}{c}\text { Quark sub-process } \\
\text { (class) }\end{array}$ & Decay mode & $\operatorname{Im} \lambda$ \\
\hline $\begin{array}{c}\bar{b} \rightarrow \bar{c}+c \bar{s}, \bar{c}+c \bar{d}, \bar{s} \\
(i)\end{array}$ & $\begin{array}{c}B_{d} \rightarrow \psi K_{S}, \chi K_{S}, \phi K_{S}, \eta_{c} K_{S}, \\
\omega K_{S}, \rho K_{S}, D^{+} D^{-}, \bar{D}^{0} D^{0}, \\
\psi K_{L}, \phi K_{L}, \rho K_{L}, \ldots\end{array}$ & $-\sin (2 \beta)$ \\
\hline $\begin{array}{c}\bar{b} \rightarrow \bar{u}+u \bar{d} \\
(i i)\end{array}$ & $\begin{array}{c}B_{d} \rightarrow \pi^{+} \pi^{-}, \bar{p} p, \rho \pi^{0}, \\
\omega \pi^{0}, \pi^{0} \pi^{0}\end{array}$ & $-\sin (2 \alpha)$ \\
\hline $\begin{array}{c}\bar{b} \rightarrow \bar{u}+u \bar{d} \\
(i i i)\end{array}$ & $\begin{array}{r}B_{s} \rightarrow \rho K_{S}, \omega K_{S}, \\
\rho K_{L}, \omega K_{L}\end{array}$ & $-\sin (2 \gamma)$ \\
\hline $\bar{b} \rightarrow \bar{c}+c \bar{s}, \bar{c}+c \bar{d}$ & $B_{s} \rightarrow \psi \phi, \eta_{c} \phi, \psi K_{S}$ & $2\left|\frac{V_{u s} V_{u b}}{V_{u d} V_{c b}}\right| \sin \gamma$ \\
\hline
\end{tabular}

We concentrate on three promising classes of measurements:

(i) Measuring $\sin (2 \beta)$ in $B_{d}$ decays:

This class has the advantage that different quark subprocesses: $\bar{b} \rightarrow \bar{c}+c \bar{s}, \bar{b} \rightarrow \bar{c}+c \bar{d}, \bar{b} \rightarrow \bar{s}$, all yield the same interference term, ${ }^{12} \operatorname{Im} \lambda=-\sin (2 \beta)$. The stan- 
dard example at the hadron level is $B_{d} \rightarrow \psi K_{S}$, with an observed $^{13} B R\left(B_{d} \rightarrow \psi K_{S}\right) \approx 3 \times 10^{-4}$. To increase statistics, one can look at many decay modes: $B_{d} \rightarrow$ $\chi K_{S}, \phi K_{S}, \rho K_{S}, \omega K_{S}, D^{+} D^{-}, \bar{D}^{0} D^{0}, \psi K_{L}, \phi K_{L}, \rho K_{L}$, etc.

(ii) Measuring $\sin (2 \alpha)$ in $B_{d}$ decays:

The relevant quark subprocess here is $\bar{b} \rightarrow \bar{u}+u \bar{d}$. Possible two-body hadronic decay modes are ${ }^{14} B_{d} \rightarrow$ $\pi^{+} \pi^{-}, \omega \pi^{0}, \rho \pi^{0}$, and $B_{d} \rightarrow \bar{p} p$. These modes may suffer from additional contributions, either from virtual intermediate states, in the form of penguin diagrams at the quark level, ${ }^{15,16}$ or from real intermediate states, i.e., rescattering effects at the hadron level. For example, ${ }^{17}$ $B_{d} \rightarrow D^{+} D^{-} \rightarrow \pi^{+} \pi^{-}$may upset the identification of $B_{d} \rightarrow \pi^{+} \pi^{-}$as a pure $\bar{b} \rightarrow \bar{u}+u \bar{d}$ transition. Although difficult to calculate quantitatively, a recent estimate ${ }^{15}$ is that these additional contributions are less than a $20 \%$ effect for class (ii) decays, and they will be neglected here. In addition, the mode $p \bar{p}$ has opposite CP-parity in the s- and p-wave final states, producing asymmetries of opposite sign.

(iii) Measuring $\sin (2 \gamma)$ in $B_{s}$ decays:

The relevant quark subprocess is, $\bar{b} \rightarrow \bar{u}+u \bar{d}$, the same as that in class $(i i)$. However, $\operatorname{Im} \lambda$ is related to a different angle of the unitarity triangle, because the interference term depends not only on the quark subprocess but on $B^{0}-\bar{B}^{0}$ mixing, which in turn involves different CKM elements for the $B_{d}$ and $B_{s}$ systems. Possible hadronic modes of this type are $B_{s} \rightarrow \rho K_{S}$ and $B_{s} \rightarrow \omega K_{S}$, although again rescattering effects may be important. $^{17}$

A fourth class utilizes the quark subprocesses in class $(i)$, but for $B_{s}$ rather than $B_{d}$ decays. The predicted interference 
term is very small, at most of order $\sin ^{2} \theta_{C} \sin \gamma$.

In addition to the three promising classes above, decays to $\mathrm{CP}$ non-eigenstates could also show large CP-violating effects, but they are not susceptible to the same clean interpretation in terms of just CKM matrix elements. This report will be restricted to the predicted $\mathrm{CP}$ asymmetries in classes $(i)-$ (iii) only.

\subsection{Constraining the Unitarity Triangle}

Now that the relevance of various $B$-decay asymmetries has been presented, we return to the unitarity triangle and the measurements which we will use to constrain it. Two of these constraints depend on loop processes: the CP-violating parameter $\epsilon$ and the $B_{d}-\bar{B}_{d}$ mixing parameter $x_{d}$. As loop processes are GIM-suppressed, the resulting constraints strongly depend on the yet-unknown mass of the top quark, $m_{t}$. The detailed analytical expressions may be found elsewhere. ${ }^{18} \mathrm{On}$ the other hand, $\left|V_{c b}\right|$ and $\left|V_{u b} / V_{c b}\right|$ are directly measurable in semileptonic $B$ decay, and thus independent of $m_{t}$.

The values of well-known quantities used here are:

$$
\begin{aligned}
f_{K} & =0.16 \mathrm{GeV} ; \\
m_{c} & =1.4 \mathrm{GeV} ; m_{B}=5.28 \mathrm{GeV} ; M_{W}=80 \mathrm{GeV} ; \\
G_{F} & =1.166 \times 10^{-5} \mathrm{GeV}^{-2} ; \\
\left|V_{u s}\right| & =\sin \theta_{C}=0.22 ;|\epsilon|=2.26 \times 10^{-3}
\end{aligned}
$$

The QCD correction factors for $\epsilon$ and $x_{d}$ are the same as those used in Ref. 18. We consider the ranges ${ }^{7}$

$$
0.036 \leq\left|V_{c b}\right| \leq 0.056
$$

and $^{19,20} 78 \mathrm{GeV} \leq m_{t} \leq 200 \mathrm{GeV}$. The constraints on the rescaled unitarity triangle are then imposed as follows: 
a. The top mass is fixed within the range given above. As examples we choose $m_{t}=80,120,160$, and $200 \mathrm{GeV}$ in Figures 2.2, 2.3, 2.4, and 2.5, respectively.

$b$. The value of $\left|V_{c b}\right|$ is fixed within the range given above. As examples we choose $\left|V_{c b}\right|=0.036,0.046$, and 0.056 in subfigures $a, b$, and $c$, respectively.

c. The constraint ${ }^{21}$

$$
0.04 \leq\left|V_{u b} / V_{c b}\right| \leq 0.16
$$

is imposed. This forces the vertex $A$ to lie between two circles centered at the vertex $C(0,0)$. In the Figures, those circles are dotted.

d. The $x_{d}$ constraint is imposed. This requires the vertex $A$ to lie between two circles (dashed in the Figures) centered at $B(1,0)$. The width of this band arises mainly from theoretical uncertainties in $B_{B} f_{B}^{2}$ and, to a lesser extent, from lifetime and mixing ${ }^{21}$ measurements:

$$
\begin{aligned}
(0.1 \mathrm{GeV})^{2} & \leq B_{B} f_{B}^{2} \leq(0.2 \mathrm{GeV})^{2} \\
1.04 \mathrm{ps} & \leq \tau_{b} \leq 1.32 \mathrm{ps} \\
0.50 & \leq x_{d} \leq 0.78
\end{aligned}
$$

$e$. The $\epsilon$-constraint is imposed. This demands that the vertex $A$ lie between the two hyperbolas (solid curves in the Figures). The width of this band arises from the theoretical uncertainty in the $B_{K}$ parameter:

$$
1 / 3 \leq B_{K} \leq 1
$$




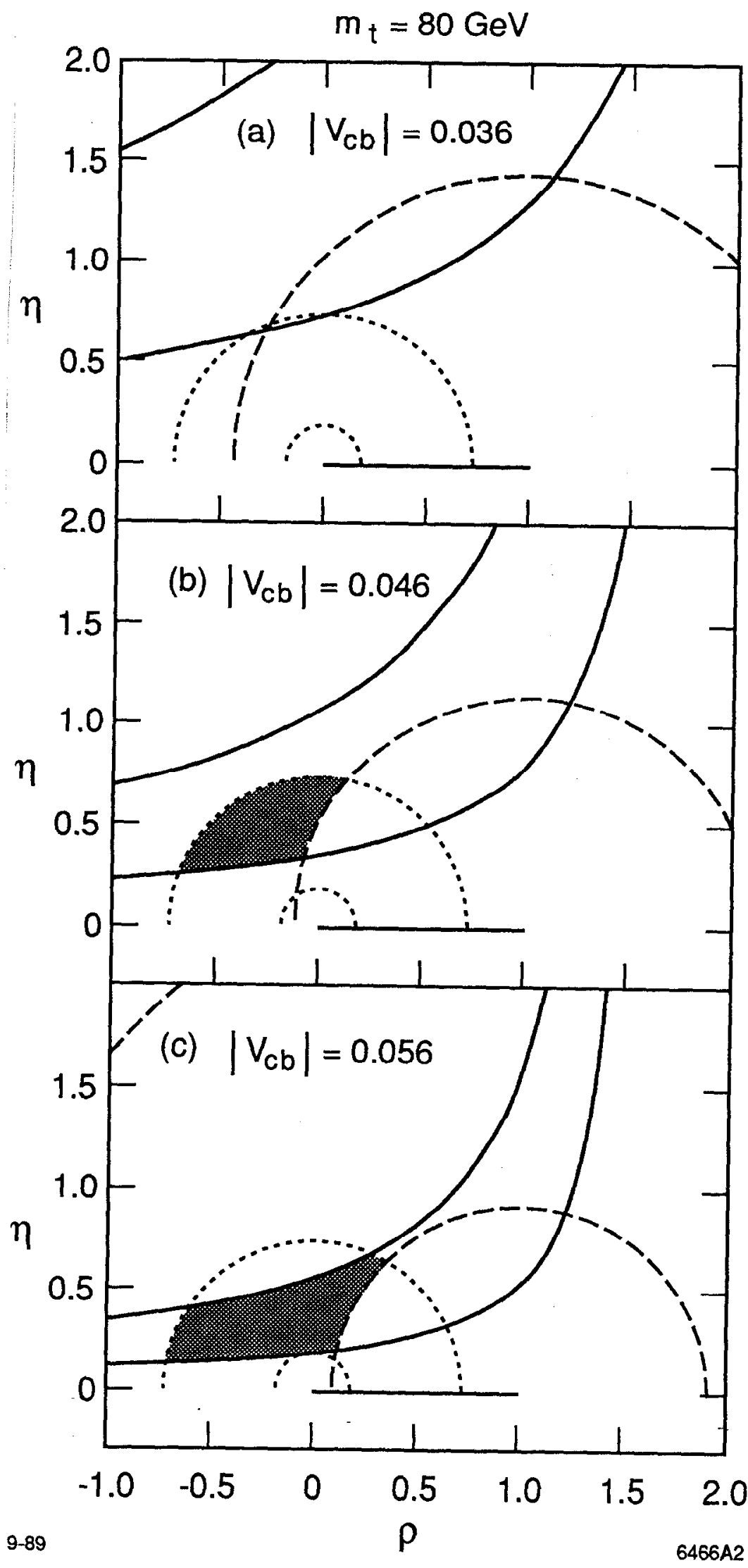

Figure 2.2 Constraints from $\left|V_{u b} / V_{c b}\right|$ (dotted circles), $x_{d}$ (dashed circles), and $\epsilon$ (solid hyperbolas) on the rescaled unitarity triangle for $m_{t}=80 \mathrm{GeV}$. The shaded region is that. allowed for the vertex $A(\rho, \eta)$. (a) $\left|V_{c b}\right|=0.036$, (b) $\left|V_{c b}\right|=$ $0.046,(c)\left|V_{c b}\right|=0.056$. 


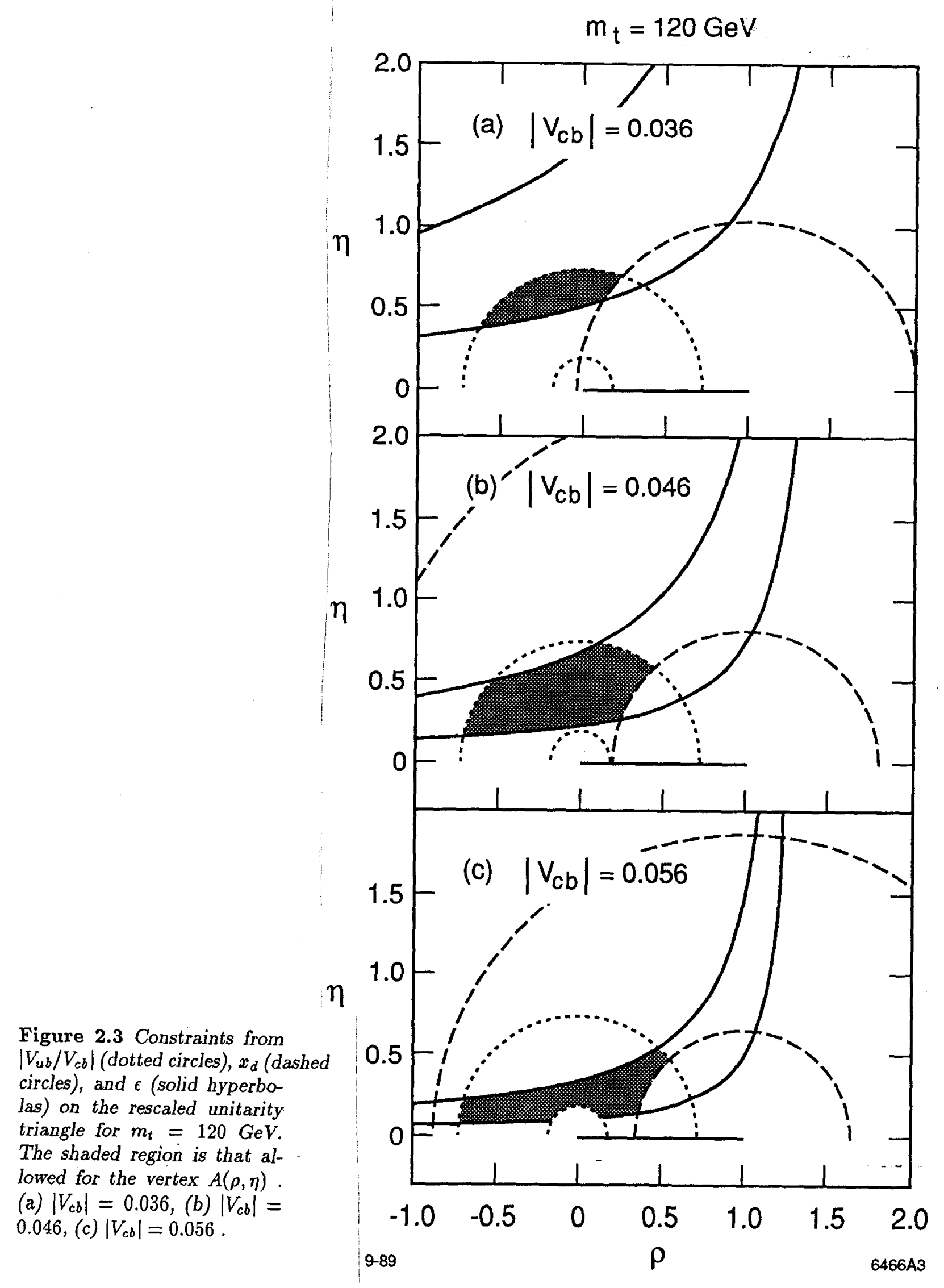




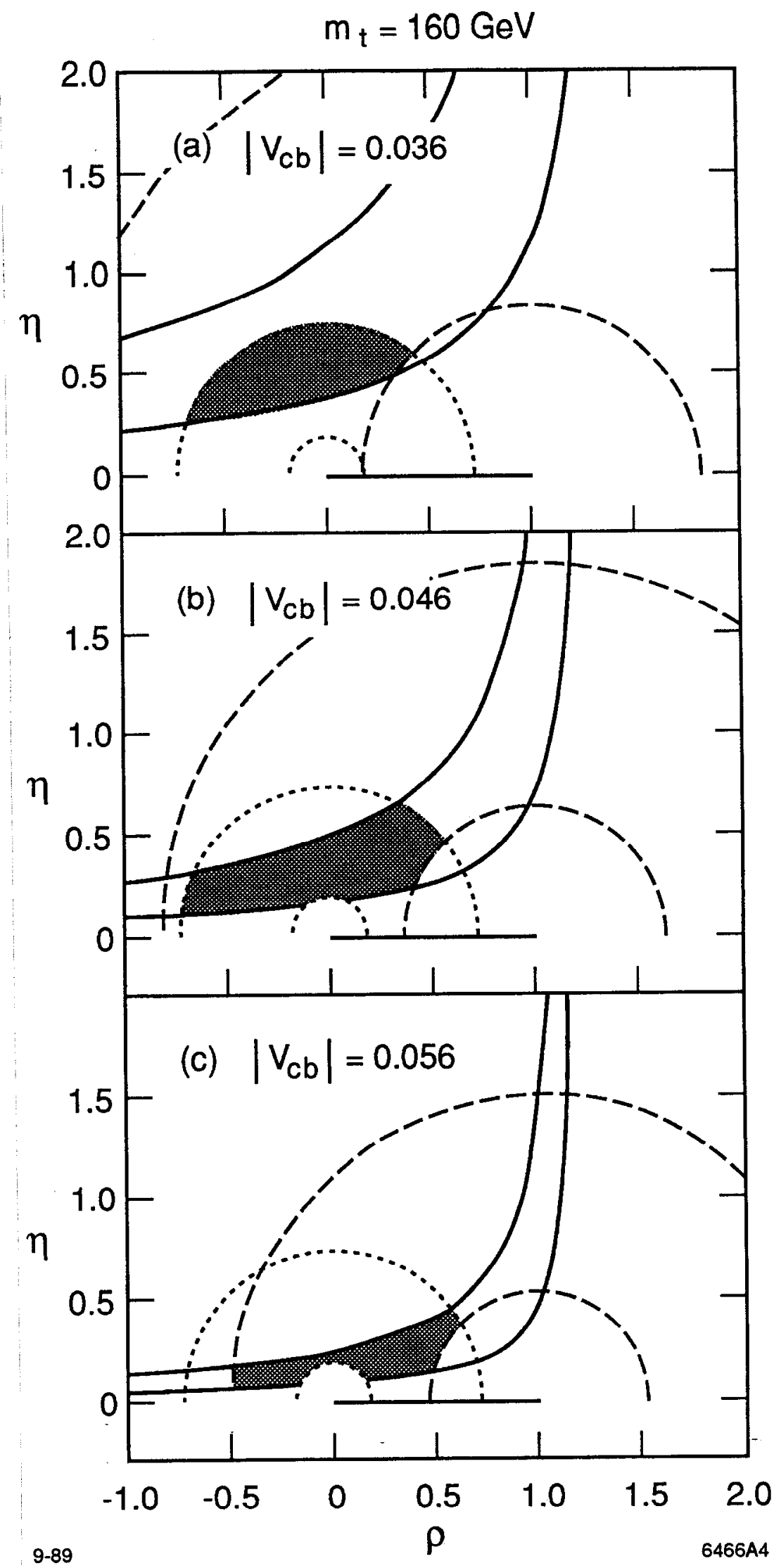

Figure 2.4 Constraints from $\left|V_{u b} / V_{c b}\right|$ (dotted circles), $x_{d}$ (dashed circles), and $\epsilon$ (solid hyperbolas) on the rescaled unitarity triangle for $m_{t}=160 \mathrm{GeV}$. The shaded region is that allowed for the vertex $A(\rho, \eta)$. (a) $\left|V_{c b}\right|=0.036$, (b) $\left|V_{c b}\right|=$ 0.046 , (c) $\left|V_{c b}\right|=0.056$. 
Figure 2.5 Constraints from $\left|V_{u b} / V_{c b}\right|$ (dotted circles), $x_{d}$ (dashed circles), and $\epsilon$ (solid hyperbolas) on the rescaled unitarity triangle for $m_{t}=200 \mathrm{GeV}$. The shaded region is that allowed for the vertex $A(\rho, \eta)$. (a) $\left|V_{c b}\right|=0.036$, (b) $\left|V_{c b}\right|=$ 0.046 , (c) $\left|V_{c b}\right|=0.056$.

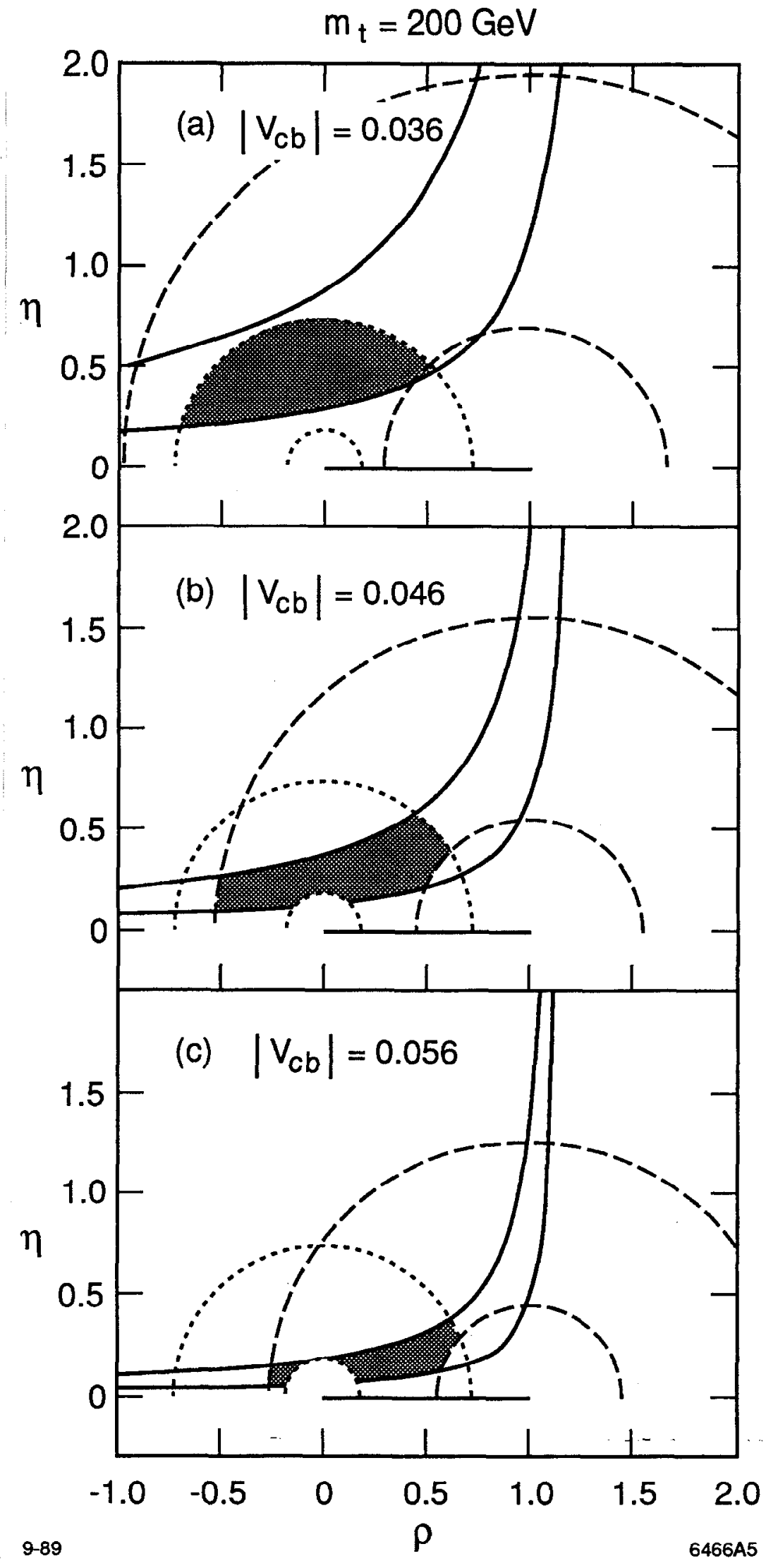


The final allowed domain for the vertex $A$ is given by the shaded region in Figures 2.2-2.5. We stress again that the $\left|V_{u b} / V_{c b}\right|$ constraint does not depend on either $m_{t}$ or $\left|V_{c b}\right|$. In contrast, the $\epsilon$ and $x_{d}$ constraints do change. Larger $m_{t}$ or larger $\left|V_{c b}\right|$ values correspond to smaller radii for the $x_{d}$ circles and, in general, to an $\epsilon$ band which is lower and narrower.

The allowed values for the angles $\alpha, \beta$, and $\gamma$ can be deduced from Figures 2.2-2.5. Figure 2.6 shows the minimum and maximum values for these angles as a function of the top mass, where the parameters range according to Eqs. (2.7)(2.11). Note that a value of $45^{\circ}$ corresponds to a maximal CP asymmetry, while $90^{\circ}$ for an angle implies that there will be no $C P$ asymmetry in the corresponding class of $B$ decays. However, if one angle is $90^{\circ}$, then $C P$ violation will necessarily exhibit itself in the other two classes. Examining Figures 2.22.5 , we see that either $\alpha$ or $\gamma$ may be $90^{\circ}$ when $m_{t} \gtrsim 80 \mathrm{GeV}$. Consequently, zero asymmetries may occur for class (ii), e.g., $B_{d} \rightarrow \pi^{+} \pi^{-}$, or class (iii), e.g., $B_{s} \rightarrow \rho K_{S}$ decays, respectively. In contrast, the angle $\beta$ ranges between

$$
2^{0}<\beta \leq \arcsin \left|V_{u b} /\left(V_{c d} V_{c b}\right)\right| \approx 47^{0}
$$

Thus, the interference term for class $(i)$, e.g., $B_{d} \rightarrow \psi K_{S}$ decays with $\operatorname{Im} \lambda=-\sin (2 \beta)$, is never zero, always negative, and can reach -1 . 
Figure 2.6 The upper and lower bounds on the angles $\alpha, \beta$, and $\gamma$ of the unitarity triangle as a function of $m_{t}$.

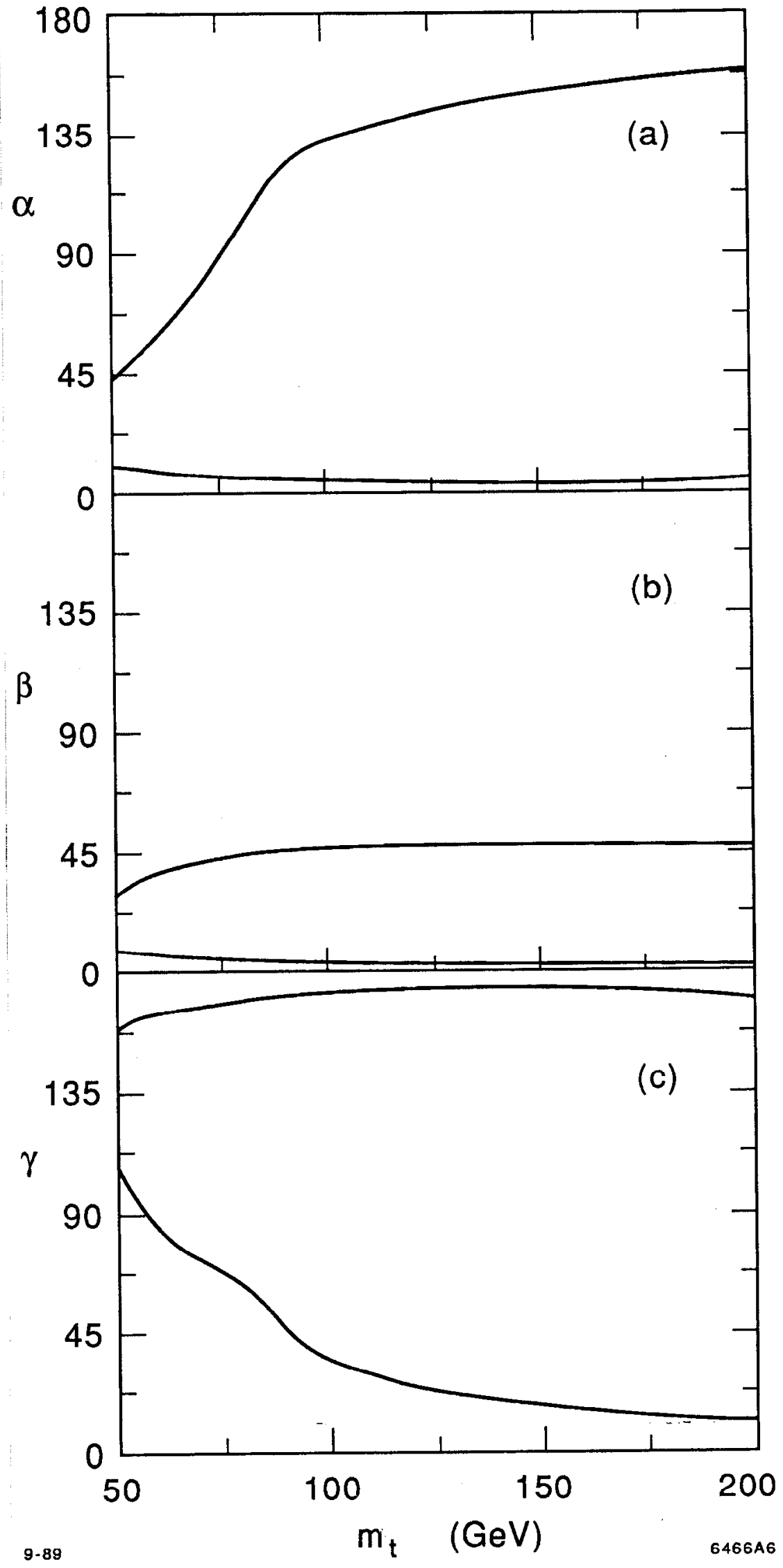




\subsection{Ranges of CP Asymmetries for $B^{0}$ Mesons}

To estimate the number of $b \bar{b}$ events required to measure $\mathrm{CP}$ violation, it is crucial to calculate the allowed range for the interference terms, $\operatorname{Im} \lambda$. The constraints of Eqs. (2.7)-(2.11) are employed. Figure 2.7 shows the minimum and maximum of $-\sin (2 \phi)$ for $\phi=\alpha, \beta, \gamma$, as a function of the top mass. The dotted line displays the lower bound on the absolute value, $|\sin (2 \phi)|$.

With $m_{t} \approx 50 \mathrm{GeV}$, large $\mathrm{CP}$ asymmetries in all three classes would be predicted (see Figure 2.7). A small top mass forces the vertex $A$ to lie in a narrow allowed region with a large imaginary part $\eta$ (due to the $\epsilon$ constraint) and with negative $\rho$ values (due to the $x_{d}$ constraint), as can be seen in Figure 2.2. With large top mass, the situation is very different. The allowed region becomes larger, and all values for the interference term of classes (ii) and (iii) are allowed,

$$
-1 \leq\{-\sin (2 \alpha) \text { or }-\sin (2 \gamma)\} \leq 1
$$

The possibilities range from maximal $(|\operatorname{Im} \lambda|=1)$ to vanishing $(|\operatorname{Im} \lambda|=0) \mathrm{CP}$ asymmetry. ${ }^{22}$

The fact that a particular interference term might vanish is disconcerting; if we were "unlucky" in the shape of the unitarity triangle chosen by nature, the failure to observe $\mathrm{CP}$ violation in just a class $(i i)$ or just a class (iii) process would not be evidence against $\mathrm{CP}$ violation originating in the CKM matrix. It is better to have a measurement for which a nonvanishing asymmetry is guaranteed. This is indeed the case for class $(i)$ processes, since the angle $\beta$ satisfies (see Figure $2.7 b)$ :

$$
-1 \leq-\sin (2 \beta) \lesssim-0.08 \text {. }
$$

Therefore, we are guaranteed that there are processes for which the magnitude of the CP-violating interference term, 
Figure 2.7 The upper and lower bounds (solid curves) for the interference term, $\operatorname{Im} \lambda$, as a function of $m_{t}$. The lower bound on . Im $\lambda \mid$, is shown as the dotted curve. (a) $\operatorname{Im} \lambda=-\sin (2 \alpha)$, (b) $\operatorname{Im} \lambda=-\sin (2 \beta)$, (c) $\operatorname{Im} \lambda=$ $-\sin (2 \gamma)$.

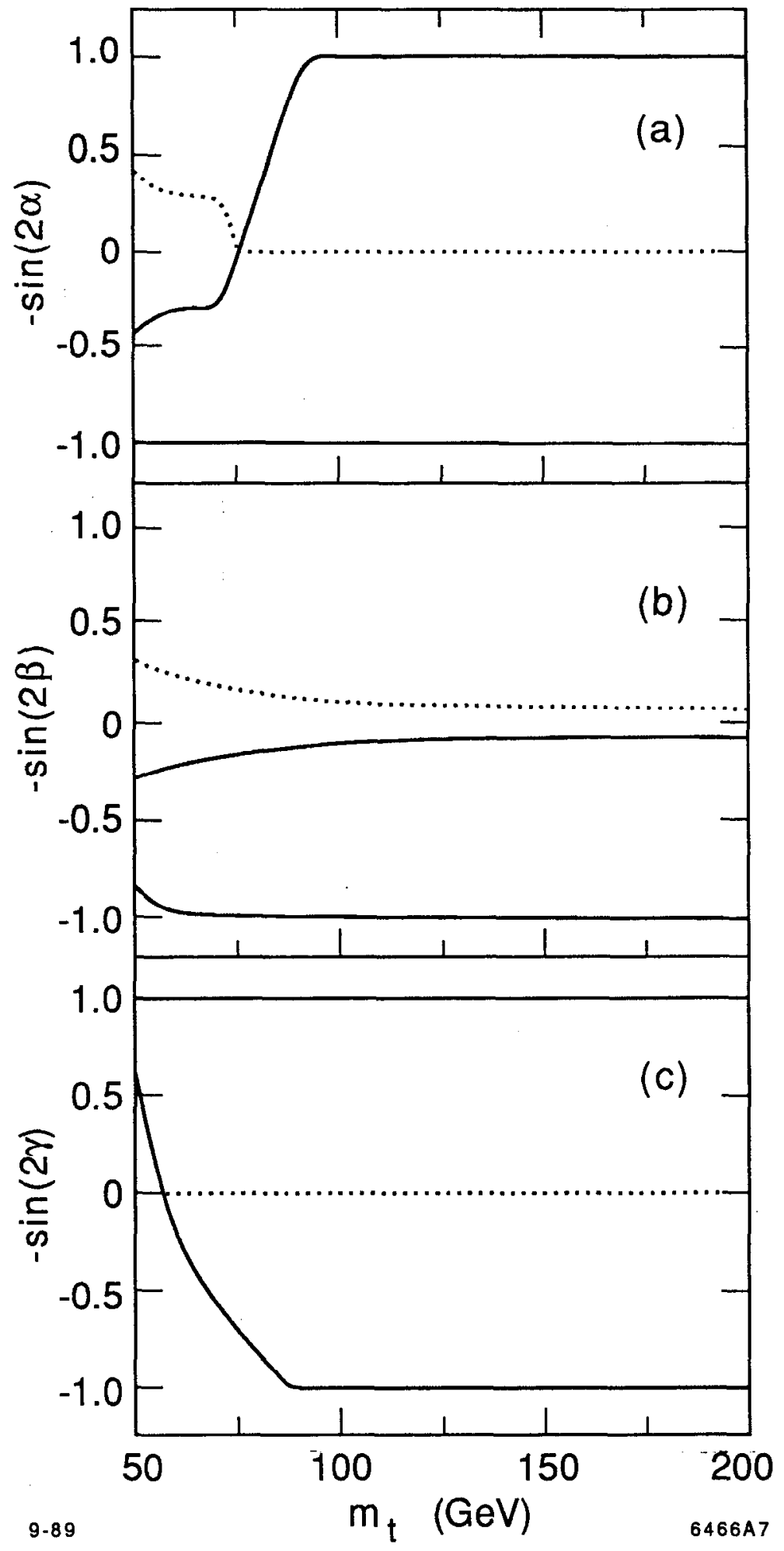


$|\operatorname{Im} \lambda|$, is greater than about 0.08 and can even be maximal. ${ }^{23}$ We define $I_{1}$ as the lower bound on $|\sin (2 \beta)|$, and present it as a function of the top mass in Figure 2.8. Can we do better from the point of view of having at least one asymmetry which is bigger than $I_{1}$ ? The answer is certainly yes if we measure processes that reside in two or three different classes, and consider the biggest value of $|\sin (2 \phi)|$ which corresponds to any of these classes.

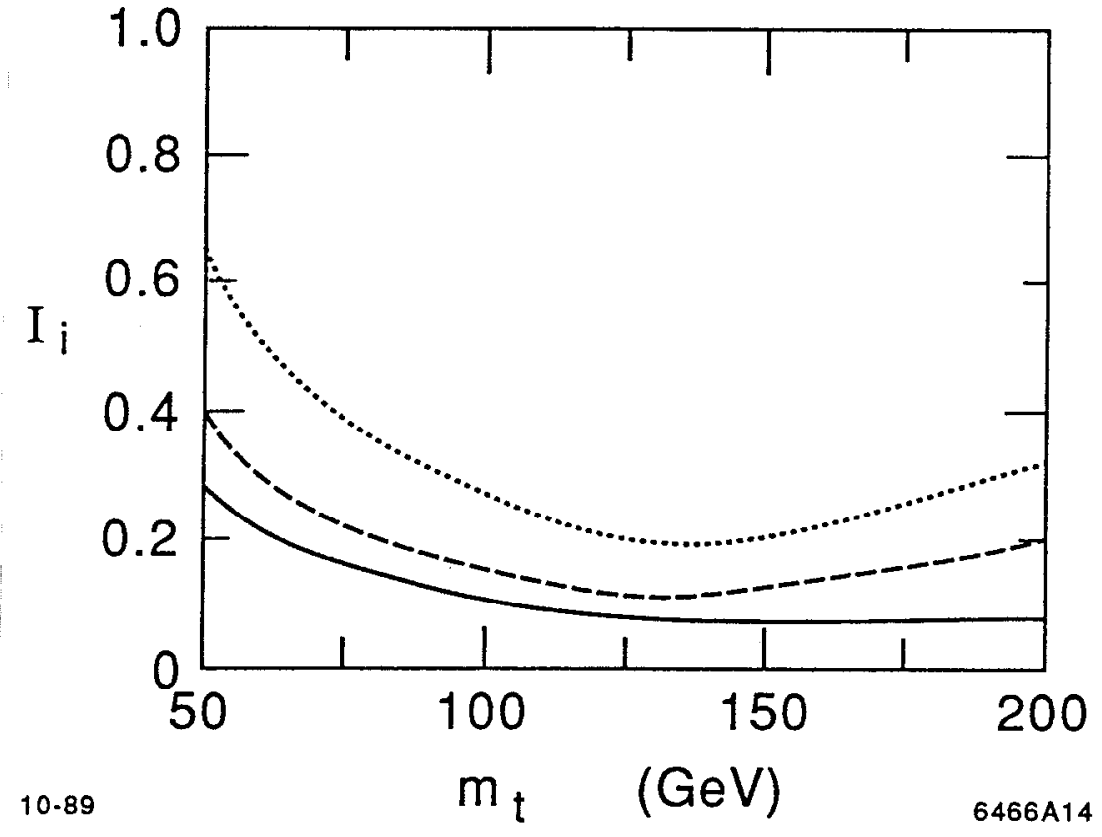

To make this quantitative, we define the following quantities for any allowed unitarity triangle $\Delta$ :

$$
\begin{aligned}
& \max _{2}(\Delta) \equiv \max \{|\sin (2 \alpha)|,|\sin (2 \beta)|\} \\
& \max _{3}(\Delta) \equiv \max \{|\sin (2 \alpha)|,|\sin (2 \beta)|,|\sin (2 \gamma)|\}
\end{aligned}
$$

If we now range over all allowed triangles, we define

$$
\begin{aligned}
& I_{2} \equiv \min _{\text {all } \Delta}\left\{\max _{2}(\Delta)\right\} \\
& I_{3} \equiv \min _{\text {all } \Delta}\left\{\max _{3}(\Delta)\right\}
\end{aligned}
$$

What is the significance of $I_{2}$ ? An experiment which is sensitive to both class (i) and class (ii) processes is assured that
Figure 2.8 The quantities $I_{1}$ (solid curve), $I_{2}$ (dashed curve), and $I_{3}$ (dotted curve) as a function of $m_{t}$ (see text). 
$|\operatorname{Im} \lambda| \geq I_{2}$ for at least one of the two classes. Figure 2.8 shows $I_{2}$ plotted against the top mass. Small top masses $(\approx 80 \mathrm{GeV})$, or large ones $(\approx 200 \mathrm{GeV})$, have $I_{2}>0.2$. This situation would be encouraging for $\mathrm{CP}$ violation studies. In contrast, intermediate top masses $(\approx 130 \mathrm{GeV})$ allow $I_{2}$ to be just above 0.1 .

Similarly, an experiment searching simultaneously for CPasymmetries in processes of all three different classes is guaranteed to find that $|\operatorname{Im} \lambda| \geq I_{3}$ for at least one of the three classes of CP-violating asymmetries. We present $I_{3}$ as a function of the top mass in Figure 2.8. Small top masses $(\approx 80 \mathrm{GeV})$ or large ones $(\approx 200 \mathrm{GeV})$ have $I_{3}>0.3$, and intermediate top masses $(\approx 130 \mathrm{GeV})$ have a minimum value of $I_{3}$ just below 0.2 . An important conclusion is that there exists an angle $\phi=\alpha, \beta$, or $\gamma$ such that $|\sin (2 \phi)| \gtrsim 0.2$. There must be substantial CP violation in at least one of the three classes if the Standard Model is the source of CP violation.

Simple geometrical considerations lead to another point of interest. If there is a near-maximal interference term in one of the three classes, then there will be a large interference term in at least one of the other two classes. For example, for $|\sin (2 \gamma)| \approx 1$ we get $|\sin (2 \phi)| \gtrsim 0.7$ for either $\phi=\alpha$ or

$\phi=\beta$. This turns out to have important bearings on the luminosity considerations presented in section 2.5 .

\subsection{Luminosity CONSIDERATIONS}

We now proceed to apply the results of the last section to find the luminosity required to observe a statistically significant CP-violating asymmetry at an electron-positron $\mathrm{B}$ factory. We choose a "favorite" $B^{0}$ decay mode that corresponds to each of the three classes of asymmetry measurements, estimate the relevant experimental and detector-related numbers that are associated with each of these decays, and then 
combine them with the magnitude of the appropriate CPviolating interference term to estimate the luminosity required for a $3 \sigma$ effect. One must always be aware that much of the experimental and detector-related input to these calculations is based on estimates or educated guesses; they may change with future data when specific branching ratios are measured, and other decay modes than we have chosen, or combinations of them, may well turn out to be optimal.

We limit our discussion to asymmetric machines running at the $\Upsilon(4 S)$, and to polarized $Z^{0}$ machines. For each type of machine, we will quote two values of integrated luminosity, $\mathcal{L}_{u}$ and $\mathcal{L}_{d}$, corresponding to the minimal and maximal magnitude of the interference term, $|\sin (2 \phi)|$, respectively. An experiment which is capable of acquiring integrated luminosity above $\mathcal{L}_{u}$ is guaranteed a statistically significant $(3 \sigma) \mathrm{CP}$-violating asymmetry in the Standard Model. On the other hand, an experiment with an integrated luminosity bclow $\mathcal{L}_{d}$ is not expected to observe a CP-violating asymmetry. Thus, observation of an effect in the latter case would indicate a source outside the Standard Model, while if no significant asymmetry is observed it will not add to our knowledge of the Standard Model.

To compute the integrated luminosity needed to measure a CP-violating asymmetry to a given level of accuracy, we follow fairly closely the analysis and assumptions made in the Snowmass 88 report: ${ }^{24}$ The formal expression for the integrated luminosity is:

$$
\int \mathcal{L} d t=\left\{2 \sigma\left(e^{+} e^{-} \rightarrow \bar{b} b\right) f_{0} B \epsilon_{r} \epsilon_{t}[(1-2 W) d \delta(\sin 2 \phi)]^{2}\right\}^{-1}
$$

where:

$f_{0}$ is the fraction of $B^{0}$ s in the $b$-quark fragmentation;

$B$ is the product of the branching fractions to the desired 
final state $f$

$\epsilon_{r}$ is the reconstruction efficiency of the final state $f$;

$\epsilon_{t}$ is the tagging efficiency, i.e. the fraction of events in which the flavor of the $B$ which decays to $f$ can be measured;

$W$ is the fraction of incorrect tags;

$d$ is a dilution factor which takes into account the loss in asymmetry due to fitting, time integration, and/or the mixing of the tagged decay;

$\delta(\sin 2 \phi)$ is the required accuracy on the CP asymmetry parameter $\sin (2 \phi)$, taken to be $|\sin (2 \phi) / 3|$ for a $3 \sigma$ effect. ${ }^{25}$

Table 2.2 lists the branching ratios and reconstruction efficiencies for the modes in each of the three different classes which we consider.

Table 2.2

Branching Ratios and Reconstruction Efficiencies for Representative Decay Modes of the Three Classes

\begin{tabular}{|c|c|c|c|c|}
\hline Class & Decay mode & $B$ & $\epsilon_{r}$ (asym. $\left.\Upsilon(4 S)\right)$ & $\epsilon_{r}\left(\right.$ pol. $\left.Z^{0}\right)$ \\
\hline$(i)$ & $B_{d} \rightarrow \psi K_{S}$ & $\left(3 \times 10^{-4}\right) \times 0.14$ & 0.61 & 0.46 \\
\hline$(i i)$ & $B_{d} \rightarrow \pi^{+} \pi^{-}$ & $2 \times 10^{-5}$ & 0.8 & 0.8 \\
\hline$(i i i)$ & $B_{s} \rightarrow \rho K_{S}$ & $3 \times 10^{-5}$ & - & 0.46 \\
\hline
\end{tabular}

The rate ${ }^{13}$ for the mode $B_{d} \rightarrow \psi K_{S}$ is a factor of 0.6 times that ${ }^{24}$ used in the Snowmass 88 report. The modes $B_{d} \rightarrow \pi^{+} \pi^{-}$and $B_{s} \rightarrow \rho K_{S}$ have yet to be observed, and estimates of their branching ratios depend on uncertain hadronic matrix elements and $\left|V_{u b} / V_{c b}\right|$. As working values, we use branching ratios of $2 \times 10^{-5}$ for $B_{d} \rightarrow \pi^{+} \pi^{-}$and $3 \times 10^{-5}$ for $B_{3} \rightarrow \rho K_{S}$. The latter, in particular, might be thought optimistic, but, as will be seen shortly, even this branching ratio will not help to lower the required luminosities. 
The reconstruction efficiencies in Table 2.2 should be achievable, at least within a factor of two, by state-of-the-art detectors. ${ }^{26}$ Table 2.3 summarizes the characteristics of $B$ production and tagging at the two machines which are relevant to Eq. (2.17) . Combinations of branching ratios and tagging efficiencies which are higher than given here will result in a lower required luminosity, and vice versa.

\section{Table 2.3}

Comparison between the Asymmetric $\Upsilon(4 S)$ and the Polarized $Z^{0}$

\begin{tabular}{|c|c|c|}
\hline Factor & Asymmetric $\Upsilon(4 S)$ & Polarized $Z^{0}$ \\
\hline$\sigma\left(e^{+} e^{-} \rightarrow \bar{b} b\right)(n b)$ & 1.2 & 6.3 \\
\hline Fraction of $B^{0}, f_{0}$ & $0.5\left(\right.$ for $\left.B_{d}\right)$ & $\begin{array}{c}0.35\left(\text { for } B_{d}\right) \\
0.15\left(\text { for } B_{s}\right)\end{array}$ \\
\hline Tag efficiency, $\epsilon_{t}$ & 0.48 & 0.61 \\
\hline Wrong tag fraction, $W$ & 0.08 & 0.125 \\
\hline Asymmetry dilution, $d$ & $0.61\left(\right.$ for $\left.B_{d}\right)$ & $\begin{array}{c}0.61\left(\text { for } B_{d}\right) \\
0.50\left(\text { for } B_{s}\right)\end{array}$ \\
\hline
\end{tabular}

\subsection{Asymmetric Machine Operating at the $\Upsilon(4 S)$}

We update the fraction of neutral $B_{d}$ mesons at the $\Upsilon(4 S)$ to be 0.5 (rather than the value 0.43 used at Snowmass 88) from the recent measurements by ARGUS and CLEO collaborations, ${ }^{21}$ and take the tagging efficiency to be $48 \%$ when the charges of both the kaons and the leptons from the accompanying $B$ are used. The solid curves in Figure 2.9 show $\mathcal{L}_{u}$ and $\mathcal{L}_{d}$ for the class $(i)$ process $B_{d} \rightarrow \psi K_{S}$ alone as a function of the top mass. To observe the smallest possible interference term, $|\sin (2 \beta)|$, at the $3 \sigma$ level, the required integrated luminosity is

$$
\mathcal{L}_{u} \approx 4 \times 10^{41} \mathrm{~cm}^{-2}
$$


Figure 2.9 The integrated luminosity of an asymmetric electron-positron collider operating at the $\Upsilon(4 S)$ required to observe a statistically significant $(3 \sigma) C P$-violating asymmetry as a function of $m_{t}$. Minimum and maximum required integrated luminosity when $C P$-violating asymmetries are searched for in the decay $B_{d} \rightarrow \psi K_{S}$ (solid curve), or simultaneously in the decays $B_{d} \rightarrow \psi K_{S}$ and $B_{d} \rightarrow$ $\pi^{+} \pi^{-}$(dashed curve). The minimum integrated luminosity curves in these two cases are identical. for $m_{t} \gtrsim 130 \mathrm{GeV}$. It is lower for a lighter top. At the other extreme, if the interference term is maximal, then an inte-

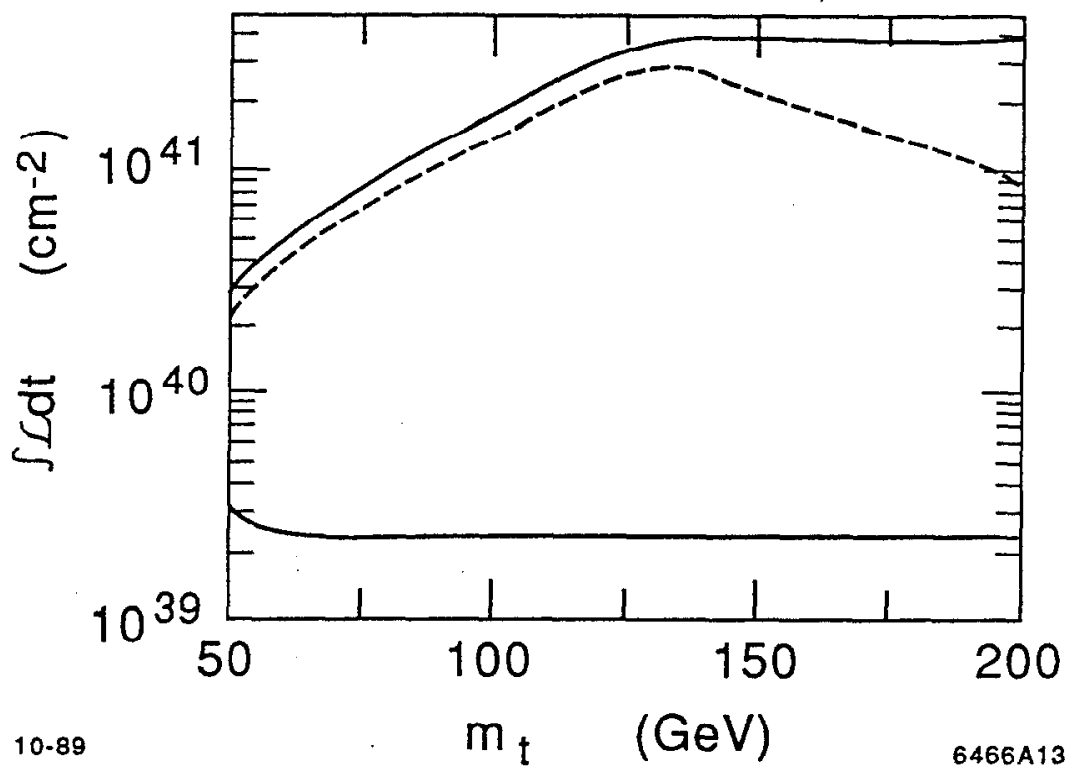

grated luminosity $\mathcal{L}_{d} \approx 2-3 \times 10^{39} \mathrm{~cm}^{-2}$ will suffice.

An experiment which is sensitive to both class $(i)$ and class (ii) processes would require a smaller integrated luminosity to see a statistically significant effect. In much the same way that we defined $I_{2}$ in the previous section, we define $\mathcal{L}_{u 2}$ and $\mathcal{L}_{d 2}$ by ranging over all allowed triangles ${ }^{27}$ for a combination of the class $(i)$ and class (ii) processes $B_{d} \rightarrow \psi K_{S}$ and $B_{d} \rightarrow \pi^{+} \pi^{-}$. Thus, $\mathcal{L}_{u 2}$ is the integrated luminosity which guarantees in the Standard Model an observation of a CP-violating asymmetry at the $3 \sigma$ level, if asymmetries in both classes $(i)$ and (ii) are measured. The dashed curves in Figure 2.9 show $\mathcal{L}_{u 2}$ and $\mathcal{L}_{d 2}$ as a function of the top mass. We find that

$$
\mathcal{L}_{u 2} \approx 3 \times 10^{41} \mathrm{~cm}^{-2}
$$

for $m_{t} \approx 130 \mathrm{GeV}$. This is not much below the value of $\mathcal{L}_{u}$ given previously. $\mathcal{L}_{u 2}$ drops below $10^{41} \mathrm{~cm}^{-2}$ only if the top is lighter than $90 \mathrm{GeV}$ or heavier than $200 \mathrm{GeV}$. If the values of the interference terms are favorable, an integrated 
luminosity of $\mathcal{L}_{d 2} \approx 2-3 \times 10^{39} \mathrm{~cm}^{-2}$ will suffice, as was the case for class $(i)$ processes alone.

The addition of class (ii) processes has not changed much. Could we have lower required luminosities if we simultaneously search for CP-violating asymmetries in all three classes? An $e^{+} e^{-}$collider could run at the $\Upsilon(5 S)$ to study $B_{s}$ decays which fall in class (iii). However, lower cross-section, lower tagging efficiencies and low hadronization of a $b$-quark into a $B_{s}$-meson make this possibility unattractive. We find that a simultaneous measurement of processes in all three classes does not lower the required luminosity.

\subsection{POLARIZED $Z^{0}$}

We consider a $Z^{0}$ machine with a $90 \%$ longitudinally polarized electron and/or positron beam. The tagging of $B^{0}$ versus $\bar{B}^{0}$ mesons can be done geometrically via the forwardbackward asymmetry. ${ }^{28}$ This, together with a large crosssection makes it an interesting alternative to the asymmetric $\Upsilon(4 S)$ machine. Since a polarized $Z^{0}$ machine is automatically a source of $B_{s}$ mesons, we consider situations where (1) the detector is sensitive to only class $(i)$ processes, $(2)$ the detector is sensitive to both class $(i)$ and (ii) processes, and (3) the detector is sensitive to all three classes simultaneously.

The results for detection of only class (i) decays are shown in Figure 2.10, where $\mathcal{L}_{u}$ and $\mathcal{L}_{d}$ are presented as a function of the top mass. The results are smaller by a factor of 2.8 than those for the asymmetric $\Upsilon(4 S)$ machine (see the analogous Figure 2.9). A $90 \%$ polarized $Z^{0}$ machine needs

$$
\mathcal{L}_{u} \approx 1.4 \times 10^{41} \mathrm{~cm}^{-2}
$$

in order to be guaranteed a $3 \sigma \mathrm{CP}$-violating asymmetry in the mode $B_{d} \rightarrow \psi K_{S}$ within the Standard Model. The minimum luminosity to see a significant effect is $\mathcal{L}_{d} \approx 10^{39} \mathrm{~cm}^{-2}$. 
Figure 2.10 The integrated luminosity of an electronpositron collider operating at the $Z$ with a $90 \%$ polarized beam required to observe a statistically significant $(3 \sigma) \mathrm{CP}$ violating asymmetry as a function of $m_{t}$. Minimum and maximum required integrated Juminosity when $C P$-violating asymmetries are searched for in the decay $B_{d} \rightarrow \psi K_{S}$ (solid curve), or simultaneously in the decays $B_{d} \rightarrow \psi K_{S}$ and $B_{d} \rightarrow \pi^{+} \pi^{-}$(dashed curve). The dashed curves apply as well when CP-violating asymmetries in the three decays, $B_{d} \rightarrow \psi K_{S}, B_{d} \rightarrow \pi^{+} \pi^{-}$, and $B_{s} \rightarrow \rho K_{S}$ are simultaneously searched for.
While individually different, the ratio of required luminosities between the asymmetric $\Upsilon(4 S)$ and polarized $Z^{0}$ machines is very close to that found in Ref. 24.

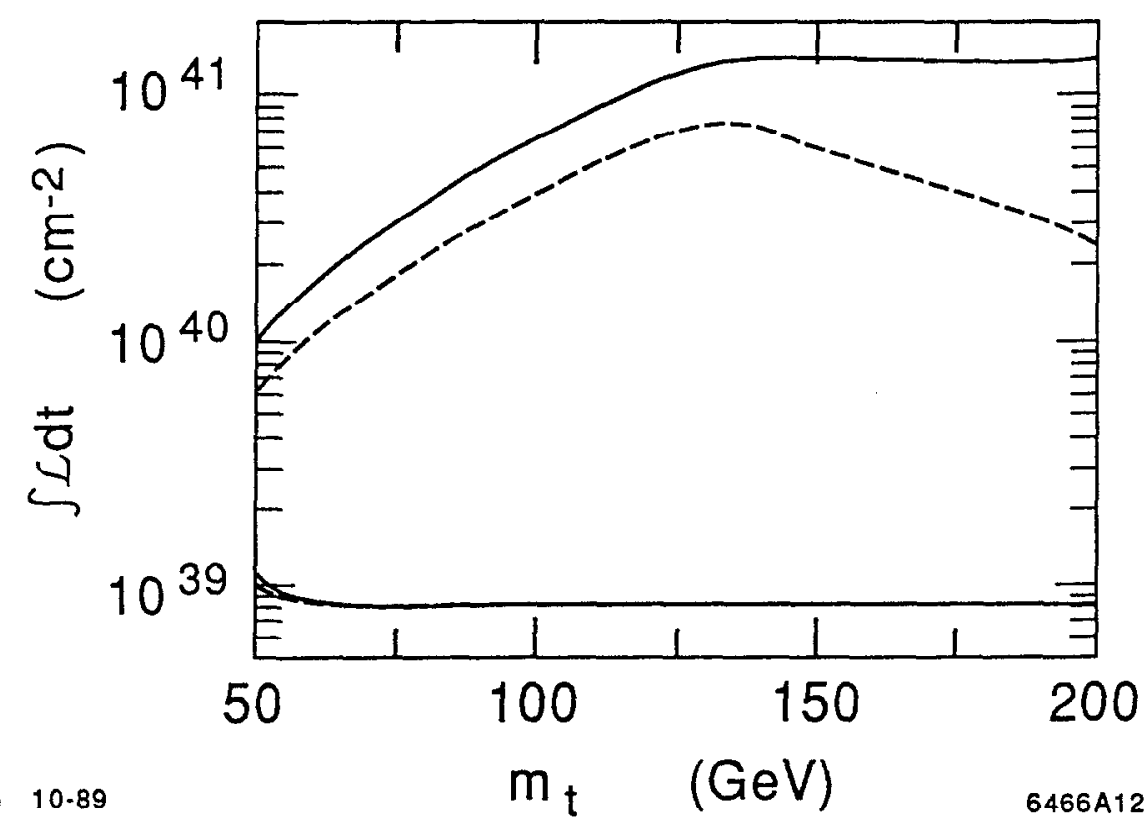

The luminosity required will be less if we are in situation (2). The argument follows exactly the same lines as for the $\Upsilon(4 S)$ machine, and the results are shown in Figure 2.10 (compare to Figure 2.9). We find

$$
\mathcal{L}_{u 2} \approx 8 \times 10^{40} \mathrm{~cm}^{-2}
$$

for $m_{t} \approx 130 \mathrm{GeV}$. For either smaller or larger top masses, the required luminosity would be less, e.g., $3 \times 10^{40} \mathrm{~cm}^{-2}$ for $m_{t} \approx$ $200 \mathrm{GeV}$. The minimal luminosity for a useful experiment is still $\mathcal{L}_{d 2} \approx 10^{39} \mathrm{~cm}^{-2}$, as was the case for $\mathcal{L}_{d}$.

Could we do better if we include in addition the measurement of a class (iii) decay asymmetry? If we move to situation (3) above, then we need to range over all possible allowed unitarity triangles while considering the luminosity required to see a statistically significant asymmetry in $B_{d} \rightarrow \psi K_{S}, B_{d} \rightarrow \pi^{+} \pi^{-}$, and $B_{s} \rightarrow \rho K_{S}$ decays within 
the Standard Model. In analogy to the previous situations, we define $\mathcal{L}_{u 3}$ and $\mathcal{L}_{d 3}$ to be the maximum and minimum integrated luminosity which is required to see a statistically significant asymmetry. The question that we asked above can be rephrased into: is $\mathcal{L}_{u 3}$ significantly smaller than $\mathcal{L}_{u 2}$ ? This could be the case if the product $|\sin (2 \phi)|^{2} \cdot\left(f_{0} B \epsilon_{r} \epsilon_{t} d^{2}\right)$ was larger for the $B_{\boldsymbol{s}} \rightarrow \rho K_{S}$ mode than for the other two [see Eq. (6.1)]. The answer is given in Figure 2.10, where both $\mathcal{L}_{u 2}$ and $\mathcal{L}_{u 3}$ (as well as $\mathcal{L}_{d 2}$ and $\mathcal{L}_{d 3}$ ) are displayed as the same dashed curve. As mentioned in section 5, there is no choice of CKM parameters which makes the CP-asymmetry in $B_{s} \rightarrow \rho K_{S}$ large while rendering the asymmetries in $B_{d} \rightarrow \psi K_{S}$ and $B_{d} \rightarrow \pi^{+} \pi^{-}$both small. Given our assumptions on production cross sections, branching ratios, and efficiencies, it follows that there is no improvement with a simultaneous measurement of asymmetries in three rather than two classes.

Our conclusions regarding the $B_{s} \rightarrow \rho K_{S}$ mode for the asymmetric $\Upsilon(4 S)$ machine (operating for this purpose at the $\Upsilon(5 S)$ resonance) and the polarized $Z^{0}$ machine do not imply that a measurement of class (iii) asymmetries is useless. On the contrary, this is a very important measurement that will provide additional information on the Standard Model parameters. Whether the independently-measured three angles will sum up to $180^{\circ}$ is a stringent test for the CKM model of $\mathrm{CP}$-violation. All we conclude here is that measuring $\mathrm{CP}$ asymmetry in class (iii) processes in addition to class $(i)$ and class (ii) asymmetries will not relax the luminosity requirements for the polarized $Z^{0}$ machine; and certainly not for the asymmetric $\Upsilon(4 S)$ machine. 


\section{REFERENCES}

1. J. H. Christenson, J. W. Cronin, V. L. Fitch, and R. Turlay, Phys. Rev. Lett. $\underline{13}, 138$ (1964).

2. K. J. Foley et al., in Proceedings of the Workshop on Experiments, Detectors, and Experimental Areas for the Supercollider, Berkeley, July 7-17, 1987, edited by R. Donaldson and M. G. D. Gilchriese (World Scientific, Singapore, 1988), p. 701.

3. J. L. Rosner, A. I. Sanda, and M. P. Schmidt, in Proceedings of the Workshop on High Sensitivity Beauty Physics at Fermilab, Fermilab, November 11-14, 1987, edited by A. J. Slaughter, N. Lockyer, and M. Schmidt (Fermilab, Batavia, 1988), p. 165; C. Hamzaoui, J. L. Rosner and A. I. Sanda, in Proceedings of the Workshop on High Sensitivity Beauty Physics at Fermilab, Fermilab, November 11-14, 1987 , edited by A. J. Slaughter, N. Lockyer, and M. Schmidt (Fermilab, Batavia, 1988), p. 215.

4. P. Krawczyk et al., Nucl. Phys. $\underline{\mathrm{B} 307}, 19$ (1988).

5. G. Belanger et al., in Proceedings of the 1988 Summer Study on High Energy Physics in the 1990s, edited by S. Jensen (World Scientific, Singapore, 1989), p. 339.

6. L.-L. Chau and W.-Y. Keung, Phys. Rev. Lett. $\underline{53}$, 1802 (1984); J. D. Bjorken, private communication and Fermilab preprint, 1988 (unpublished); C. Jarlskog and R. Stora, Phys. Lett. 208B, 268 (1988); J. L. Rosner, A. I. Sanda, and M. P. Schmidt, Ref. 3; C. Hamzaoui, J. L. Rosner and A. I. Sanda, Ref. 3.

7. Particle Data Group, Phys. Lett. 204B, 1 (1988):

8. J. D. Bjorken, private communication; J. L. Rosner, A. I. Sanda, and M. P. Schmidt, Ref. 3; C. Hamzaoui, J. 
L. Rosner and A. I. Sanda, Ref. 3; I. I. Bigi et al., in CP Violation, edited by C. Jarlskog (World Scientific, Singapore, 1989), p. 175; A. E. Blinov, V. A. Khoze, and N. G. Uraltsev, Int. J. Mod. Phys. $\underline{\text { A4, }} 1933$ (1989); K. R. Schubert, Karlsruhe preprint IEKP-KA/88-4, 1988 (unpublished).

9. L. Wolfenstein, Phys. Rev. Lett. 1ㅣ, 1945 (1983).

10. I. Dunietz and J. L. Rosner, Phys. Rev. D34, 1404 (1986) and references therein.

11. I. Bigi and A. Sanda, Nucl. Phys. B281, 41 (1987).

12. For the final states with an $\bar{s}$ quark, the quark level process is assumed to be followed by $\bar{s} \leftrightarrow \bar{d}$ through $K^{0}-\bar{K}^{0}$ mixing.

13. D. Kreinick, invited talk on results from the CLEO experiment, at the 1989 International Symposium on Lepton-Photon Interactions at High Energies, Stanford, August 6 - 12, 1989 (unpublished).

14. We thank S. Wagner for his comments on the possibility of using decay modes involving $\omega$ mesons.

15. M. Gronau, Max Planck Institute preprint MPI-PAE/PTh27/89, 1989 (unpublished).

16. B. Grinstein, Fermilab preprint FERMILAB-PUB89/158-T, 1989 (unpublished).

17. I. Bigi, private communication.

18. Y. Nir, Nucl. Phys. B306, 14 (1988).

19. P. Sinervo, invited talk on CDF results on the search for the top quark, at the 1989 International Symposium on Lepton-Photon Interactions at High Energies, Stanford, August 6 - 12, 1989 (unpublished).

20. U. Amaldi et al., Phys. Rev. D36, 1385 (1987); G. Costa et al., Nucl. Phys. B297, 244 (1988). 
21. M. Danilov and D. Kreinick, invited talks on results from the ARGUS and CLEO experiments, respectively, at the 1989 International Symposium on Lepton-Photon Interactions at High Energies, Stanford, August 6 - 12, 1989 (unpublished). We have taken the signal in both experiments for $b \rightarrow u$ transitions as implying, in a range of models, $\left|V_{u b} / V_{c b}\right|>0.04$; and averaged the two results for $B^{0}-\bar{B}^{0}$ mixing to obtain $r_{d}=0.17 \pm 0.06$, and hence the result for $x_{d}$ in the text. With a less conservative range, $r_{d}=0.17 \pm 0.04$, our results for the luminosity requirements remain essentially unchanged.

22. This range is in agreement with that quoted by Ref. 4 , but disagrees with that in Ref. 2 .

23. Here we disagree with both Ref. 2 and Ref. 4, who quote an upper limit on the magnitude of the interference term of 0.6 .

24. G. Feldman et al., in Proceedings of the 1988 Summer Study on High Energy Physics in the 1990s, edited by S. Jensen (World Scientific, Singapore, 1989), p. 561.

25. For large values of $|\sin (2 \phi)|$ the calculation of a $3 \sigma$ effect has to be modified. We neglect this correction here.

26. We thank P. Burchat, D. Cords, N. Roe, and S. Wagner for discussions on detector efficiencies that can be envisaged for various decay modes.

27. For each triangle we define $\mathcal{L}_{2}(\Delta) \equiv \min \left\{\int d t \mathcal{L}\left(B_{d} \rightarrow\right.\right.$ $\left.\left.\psi K_{S}\right), \int d t \mathcal{L}\left(B_{d} \rightarrow \pi^{+} \pi^{-}\right)\right\}$, where $\int d t \mathcal{L}\left(B_{d} \rightarrow f\right)$ is the integrated luminosity needed to observe a $3 \sigma$ asymmetry in the decay $B_{d} \rightarrow f . \mathcal{L}_{u 2}$ and $\mathcal{L}_{d 2}$ are then the maximum and minimum, respectively, of this quantity obtained in ranging over all allowed triangles. $\mathcal{L}_{u 2}$ and $I_{2}$ do not necessarily correspond to the same set of 
values for the CKM parameters.

28. W. B. Atwood, I. Dunietz, and P. Grosse-Wiesmann, Phys. Lett. B216, 227 (1989). 
Fast detector simulation permits the generation of high statistics, and study of the effects of different boosts and detector configurations.

Certain detector effects are not simulated, and their effects on an analysis must be estimated.

\section{Monte Carlo and Detector Simulation}

$\mathrm{A}_{\text {detector response is available on the SLAC VM computer, }}^{\text {software package for simulation of } e^{+} e^{-} \text {collisions and }}$ as well as on VAXes. This package has been used in most of the studies (for which a Monte Carlo simulation was required) presented in this report.

Rationale for a fast Simulation Since one of the primary features of a $B \bar{B}$ Factory is very high luminosity, many of the physics topics discussed in subsequent sections of this report require very high statistics. In order to effectively estimate backgrounds to rare decay modes, for example, many hundreds of thousands of continuum events must be generated. In addition, many studies focus on the dependence of a measurement on a specific experimental parameter, such as the beampipe radius, vertex detector resolution, the center-ofmass boost, or the electromagnetic calorimeter resolution. We have therefore chosen to use a "fast" simulation of the detector response, in order to make it possible to generate many events in a short time, so that the analysis can be repeated with, for example, a different boost. Since an important goal of this work is to establish the required detector performance, no final detector design exists. Instead, tools are available to explore different designs, some of which may conflict. For example, a CRID will greatly enhance the particle identification capabilities for high momentum tracks, but may reduce the ability of the electromagnetic calorimeter to detect low energy photons. Where such detector decisions are relevant, each study described below will specify the choices made; the final design must represent an optimization which has not yet been attempted.

Detector effects which are not completely modeled in the fast simulation must be kept in mind by users of the code. If these omissions are important in an analysis, their effects must be estimated in another way.

1. $\pi, K$ decays in flight, and their effect on reconstruction and track parameter resolution, are not simulated. However, the parameterization of $\mu / \pi$ separation includes the possibility of decay. 
2. Actual hits and multiple scattering in the tracking system are not simulated, but instead the reconstructed track parameters are smeared appropriately.

3. The effects of beam related noise are not simulated.

4. Nuclear interactions, bremsstrahlung, and photon conversions in the detector are not simulated.

5. Particle identification with the electromagnetic calorimeter and muon rangeout detector is simulated with a crude parameterization; see below.

\subsection{Monte Carlo Event Generation}

Two Monte Carlo event generators are available for physics analyses. The first is the standard Lund Monte Carlo version 6.3 , with the addition of a subroutine to produce a particleantiparticle final state (such as $B \bar{B}$ ). Thus, the decay models available include:

1. Continuum $u, d, s, c$, together or separately.

2. $\mu$-pair or $\tau$-pair production.

3. Production of bottom-onia and decay via $g g g$ and $\gamma g g$.

4. Decay of $\Upsilon(4 S)$ to $50 \% B^{0} \bar{B}^{0}$ and $50 \% B^{+} B^{-}$.

5. Production of $B^{0} \bar{B}^{0}$ or $B^{+} B^{-}$or $B_{s} \bar{B}_{s}$ separately.

6. Both $B$ mesons can decay to a mix of all possible modes (a "cocktail"), or one $B$ can decay to a specific final state, with the recoil $B$ decaying to a mix of modes. The decays are all handled at the quark level, in the spectator quark model. The mix includes semileptonic decays (with mass suppresion for $b \rightarrow \tau^{-} \bar{\nu}_{\tau} c$ ), the chargedcurrent decays $b \rightarrow(d \bar{u}) c$ and $b \rightarrow(s \bar{c}) c$, and the QCD-induced "neutral current" decays $b \rightarrow(c \bar{u}) d$ and $b \rightarrow(c \bar{c}) s$ (the distinction is important in the Lund color-correlated string fragmentation scheme). Nonspectator decays, and $b \rightarrow u$ decays, are not generated, they may easily be included if desired. Note that Lund fragmentation results in a slightly lower charged particle multiplicity than that measured by CLEO and ARGUS. This was deemed unimportant for most studies,
The Lund Monte Carlo is available for most Physics analyses. 
The BBGEN Monte Carlo is available for studies of $B \bar{B}$ mixing and $C P$ violation. but work is in progress to provide more realistic decay tables for the $D$ and $B$ mesons.

In all cases, The Lund Monte Carlo simulates initial state radiation, and performs the boost of all particles to the lab frame. Most $D$ and $B$ decays are handled via Lund string fragmentation at the quark level, using Petersen fragmentation with appropriate parameters; the user can request specific decay modes if desired. Finally, decay vertices are produced for all long-lived particles after the event is generated.

The second available event generator is the BBGEN package by Roy Aleksan. This package is capable of explicitly simulating $B \bar{B}$ mixing and $C P$ violation with the complete decay time dependence, for $C P+1$ and $-1 B \bar{B}$ eigenstates. One $B$ meson then decays to the desired final state, such as a CP eigenstate, while the other decays via a Lund-like mix of modes. Since the Lund MC generates all decays without regard to decay length, it is difficult to modify it to simulate $B \bar{B}$ mixing oscillations; so to explicitly see mixing in the $\mathrm{MC}$, BBGEN is used.

\subsection{Detector Simulation}

Both of the above event simulation packages are interfaced to the same fast detector simulation package. The user has available to him the generated 4-vectors of the stable and unstable particles, the track parameters of the stable charged tracks detected in the tracking system, the energies and angles of photons detected in the electromagnetic calorimeter, and information for each charged track from the particle identification systems. The generic design of each of these detector systems is described below. A "cartoon" overview of the detector is given in Figure 3.1. 


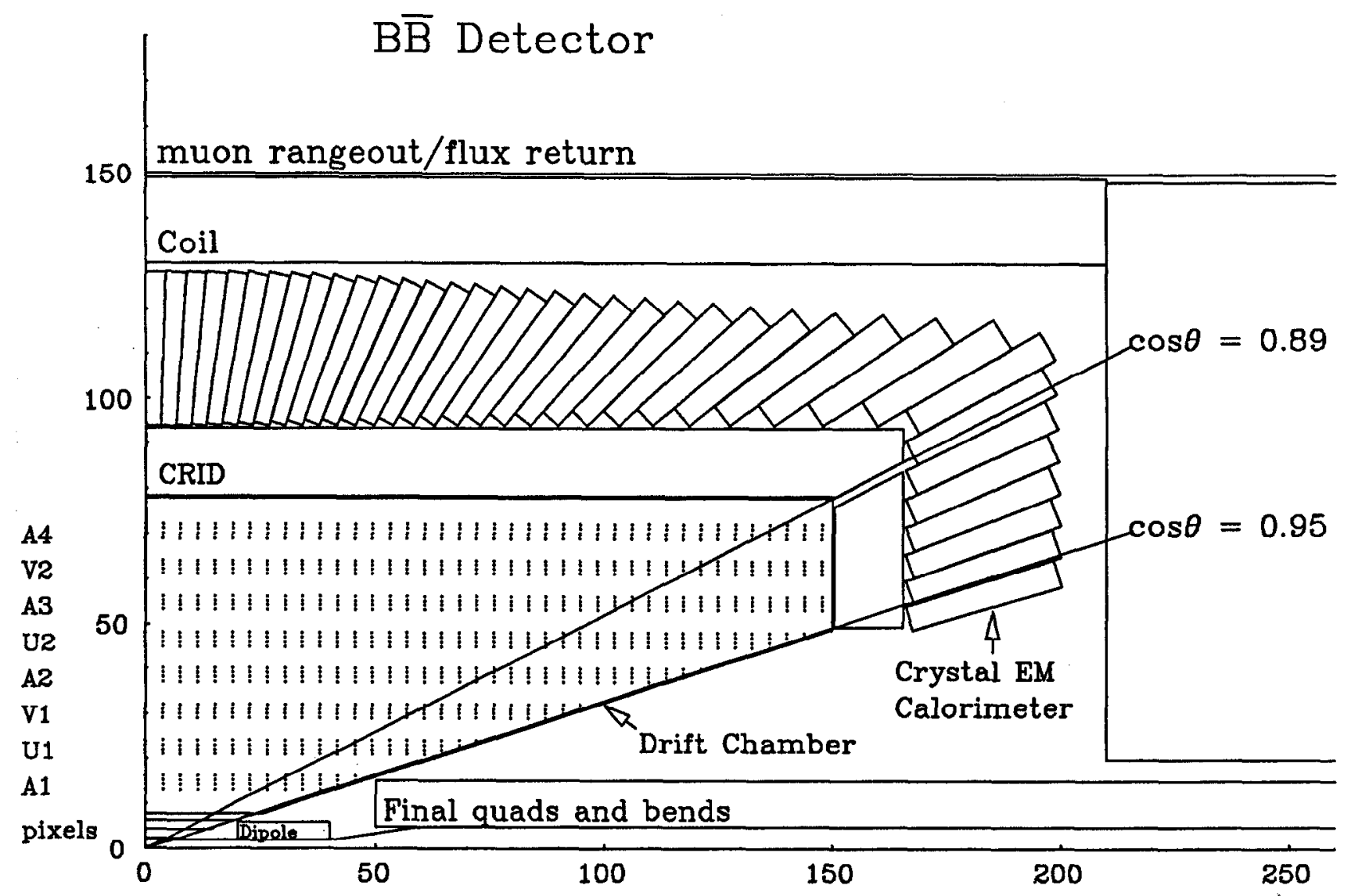

Figure 3.1 Overview of the $B \bar{B}$ detector assumed for this study, shown in $r z$ projection. 
The tracking simulation covers $95 \%$ of the solid angle with $100 \%$ efficiency, and it returns smeared track parameters.

Three layers of pixel detectors contain 110 million pixels.

The drift chamber is optimized to minimize multiple scattering.

\subsection{Tracking System}

The tracking system is assumed to contain 3 layers of pixel vertex detectors, followed by a low-mass drift chamber. An example of what the system might look like, in $x y$ projection, is given in Figure 3.2. The system covers $95 \%$ of the full solid angle, and is immersed in a uniform axial magnetic field of 1 Tesla. Its goal is to provide accurate measurements of the five track parameters evaluated at the distance of closest approach of the track to the beamline:

$$
\phi_{0}, \quad \kappa \equiv 1 / p_{t}, \quad s \equiv p_{z} / p_{t}, \quad r_{0}, \quad z_{0}
$$

Tracks are detected with $100 \%$ efficiency if they have $|\cos \theta|<$ $0.95, p_{t}>0.04 \mathrm{GeV} / \mathrm{c}$, and if they are produced within $20 \mathrm{~cm}$ of the beam in $x y$ projection.

Pixel Vertex Detector The three layers of pixel vertex detectors, with barrel geometry, are positioned at radii of 25,45 , and $65 \mathrm{~mm}$. They all cover the region $|\cos \theta|<0.95$, allowing for $1 \mathrm{~cm}$ displacements of the collision from the nominal IP; the third layer extends to $\pm 20 \mathrm{~cm}$ from the IP. The thickness of each layer is assumed to be $300 \mu \mathrm{m}$ of silicon (this includes all support and readout material), although the actual detector may be half as thick. Each pixel is assumed to be $50 \mu \mathrm{m}$ square. A total of 110 million pixels, consisting of $\sim 200$ chips, are required (2700 $\mathrm{cm}^{2}$ of silicon). This system provides the dominant measurement precision for all the track parameters except the curvature $\kappa$.

Low Mass Drift Chamber The main design goals of the central drift chamber are to

- provide uniform coverage over as large a solid angle as possible, by making the chamber long in $Z$ and small in radius;

- minimize the contribution of multiple scattering to the measurement of the curvature, by using a low density gas such as helium, and through careful choice of-wire materials and cell design;

- provide good position and $\mathrm{dE} / \mathrm{dx}$ resolution, through the use of small amounts of gas additives such as $\mathrm{CO}_{2}$ 
to reduce the diffusion and isobutane to increase the primary ionization and gas gain, and through careful design of the cell electrostatic configuration;

- provide ease of pattern recognition using jet-cell geometry.

The working design geometry for the drift chamber consists of eight superlayers (four axial, two stereo U, two stereo $\mathrm{V})$, each with four sense wire layers spaced at $1 \mathrm{~cm}$. The entrance wall, at $8 \mathrm{~cm}$ radius, is instrumented with cathode z-strips, as is the outer wall at $77 \mathrm{~cm}$. The maximum length is $\pm 150 \mathrm{~cm}$, providing a coverage of $|\cos \theta|<0.95$ for 4 superlayers and $|\cos \theta|<0.89$ for all 8 superlayers (plus the pixels and inner cathode strips).

A gas candidate might be $15 \% \mathrm{CO}_{2}, 7 \%$ isobutane, $78 \%$ helium. This gas yields low mass, low diffusion, reasonable ionization, low Lorentz angle, slow unsaturated drift velocity, reasonable gain, good $\mathrm{dE} / \mathrm{dx}$ resolution, good quenching, and good wire aging properties (the precise properties of this and similar gas mixtures is under study at present).

The cell design, including electrostatics, mechanical force, and multiple scattering considerations, is also in progress. Preliminary results indicate that it is possible to come up with a mechanically stable design employing lots of thin cathode and potential wires, with uniform drift trajectories. Tilted cells may be necessary, in order to reduce the distortion of drift trajectories due to the magnetic field. With a maximum drift distance of $25 \mathrm{~mm}$, the position resolution can be kept below $150 \mu \mathrm{m}$. Wire materials of aluminum, carbon fiber, and magnesium are being considered. It should be possible to keep the total material in the chamber, including gas and wires, to below $0.1 \%$ of a radiation length.
A helium-based gas is envisioned. 


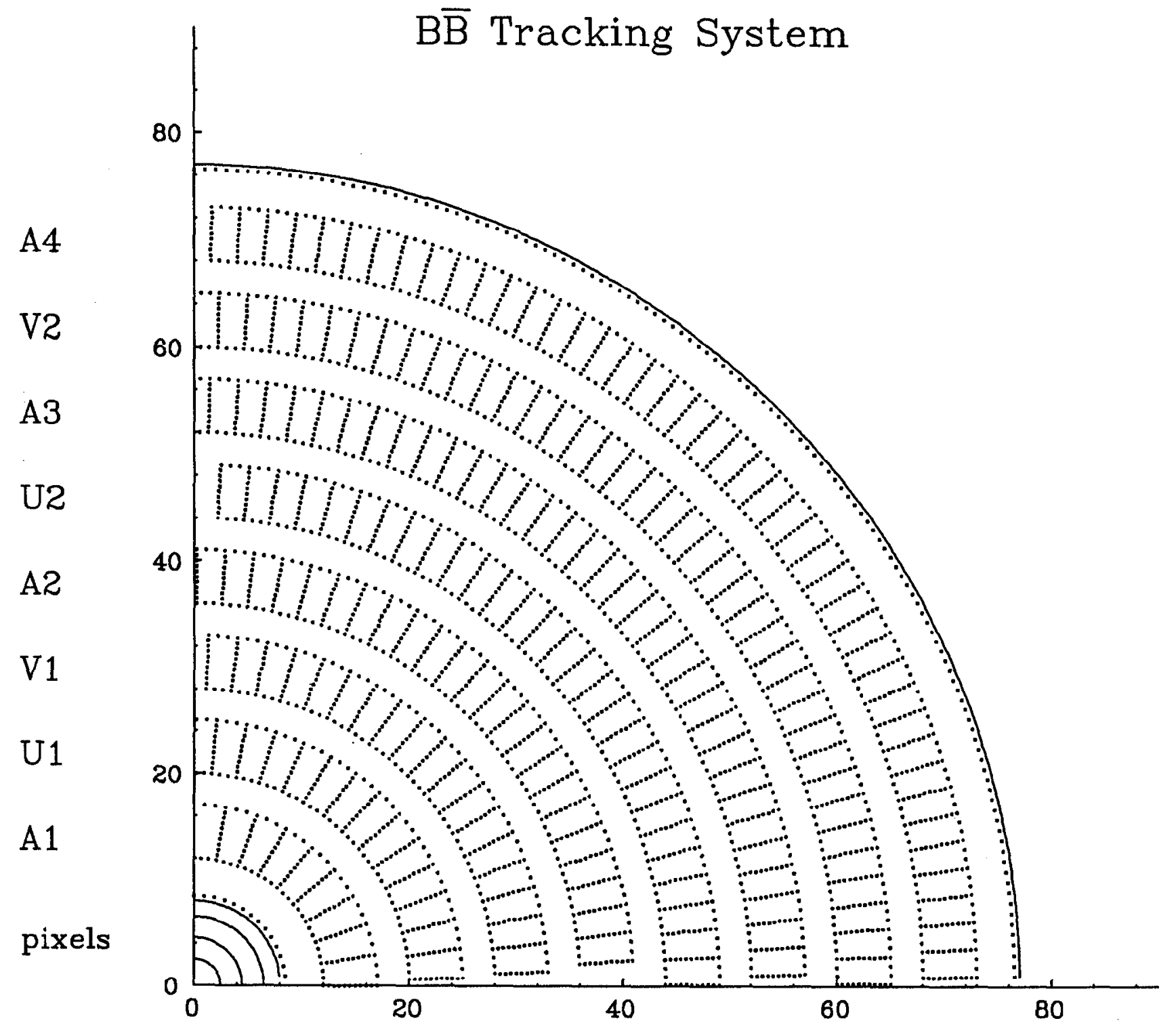

Figure 3.2 Tracking system for a $B \bar{B}$ detector, in $x y$ projection, showing dimensions (in $\mathrm{cm}$ ) and a possible cell structure. 
Beampipe and Resolution We assume a beampipe radius of $2 \mathrm{~cm}$, with a thickness of $1 \mathrm{~mm}$ beryllium equivalent (this may include coatings for synchrotron photon masking, and cooling pipes). Thus, with the geometry and measurement resolutions discussed above, the resolutions used to smear the five track parameters are given as follows:

$$
\begin{gathered}
\sigma_{\phi_{0}} \quad(m r)=\sqrt{0.22^{2}+1.12^{2} \cdot\left(\frac{1}{p \beta}\right)^{2}\left(\frac{p_{z}}{p_{t}}\right)^{3}}, \\
\sigma_{p_{t} / p_{t}^{2}} \quad(\% / G e V)=\sqrt{0.23^{2}+0.27^{2} \cdot\left(\frac{1}{p \beta}\right)^{2}\left(\frac{p_{z}}{p_{t}}\right)^{3}}, \\
\sigma_{p_{z} / p_{t}} \quad(m i l)=\sqrt{0.24^{2}+1.12^{2} \cdot\left(\frac{1}{p \beta}\right)^{2}\left(\frac{p_{z}}{p_{t}}\right)^{5}}, \\
\sigma_{r_{0}} \quad(\mu \mathrm{m})=\sqrt{15^{2}+25^{2} \cdot\left(\frac{1}{p \beta}\right)^{2}\left(\frac{p_{z}}{p_{t}}\right)^{3}}, \\
\sigma_{z_{0}} \quad(\mu \mathrm{m})=\sqrt{17^{2}+25^{2} \cdot\left(\frac{1}{p \beta}\right)^{2}\left(\frac{p_{z}}{p_{t}}\right)^{5}} .
\end{gathered}
$$

The momentum resolution due to measurement error as a function of $|\cos \theta|$ is shown in Figure 3.3.

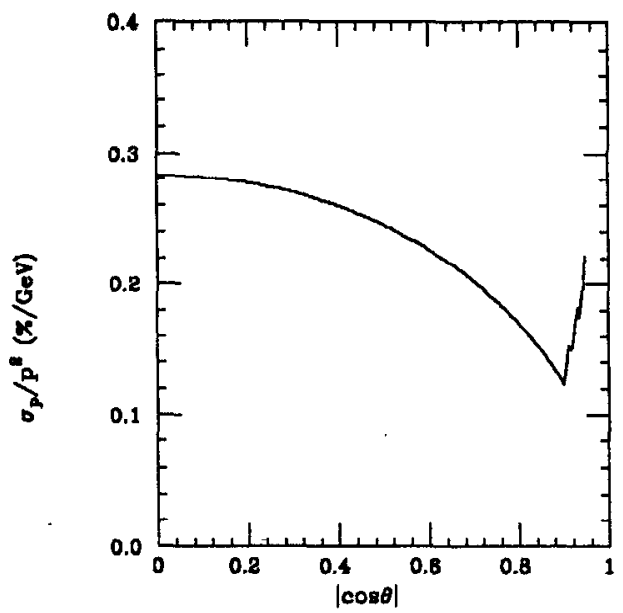

To attain this precision in the momentum resolution, one must fit for the scattering angles in the silicon detectors and
A $2 \mathrm{~cm}$ beampipe is assumed. 
The momentum resolution is achieved by fitting for scattering angles in the silicon.
Approximately 12000 CsI crystals comprise the electromagnetic calorimeter. chamber walls. It may also be possible to improve on this resolution by identifying and fitting for scattering angles in the wires hit by the tracks (often, no wires at all will be hit by a track). Note also that tracks with $|\cos \theta|>0.89$ will not pass through the outer layers of the drift chamber, so the momentum resolution is degraded by the appropriate factor. A $5 \times 5$ track parameter error matrix (assumed diagonal), based on detected track parameters, is made available for vertex fitting.

\subsection{Electromagnetic Calorimetry}

With the small outer radius of the drift chamber $(78 \mathrm{~cm})$, it is possible to include a barrel CRID and still keep the inner radius of a barrel calorimeter below one meter, making a crystal calorimeter affordable. We leave $16 \mathrm{~cm}$ for a CRID; thus 18 radiation lengths of CsI would lie from 94 to $128 \mathrm{~cm}$ (followed by the coil and a muon rangeout detector). The endcap calorimeters, with outer radius of $94 \mathrm{~cm}$ and inner radius of $54 \mathrm{~cm}(|\cos \theta|<0.95)$ would lie between $166 \mathrm{~cm}$ $<|z|<200 \mathrm{~cm}$. The total volume of CsI would be 7900 liters in the barrel and 1265 liters for the two endcaps. We may expect a cost of $\$ 1000 /$ liter for the crystal.

We have assumed an angular resolution of $10 \mathrm{mr}$ for both $\theta$ and $\phi$. The minimum dimension of a crystal would then have to be $3.25 \mathrm{~cm}$, which is approximately the Moliere radius in CsI; thus, showers can be fully contained in one crystal, but will often cross into neighboring crystals, improving the angular resolution. There will be close to 11000 crystals in the barrel and 1300 in the two endcaps.

The simulation assumes $100 \%$ detection efficiency for photons with $|\cos \theta|<0.95$ and $E_{\gamma}>0.025 \mathrm{GeV}$. The energy resolution is assumed to be $\sigma_{E} / E=2 \% / E^{1 / 4} \oplus 1 \%$, where the $\oplus$ indicates that the two terms are added in quadrature. The angular resolution is taken to be $10 \mathrm{mr}$ in $\phi$ and $\theta$. In addition, $K_{L}, n, \bar{n}$ will interact in the calorimeter with a probability of $1-e^{-\lambda}$, where $\lambda$-is a nuclear absorption length $(=$ 18 radiation lengths in $\mathrm{CsI}$ ). Such an interaction will result in a shower, indistinguishable from a photon, with an energy randomly chosen between 0 and $E_{\text {had }}$. 
(a)

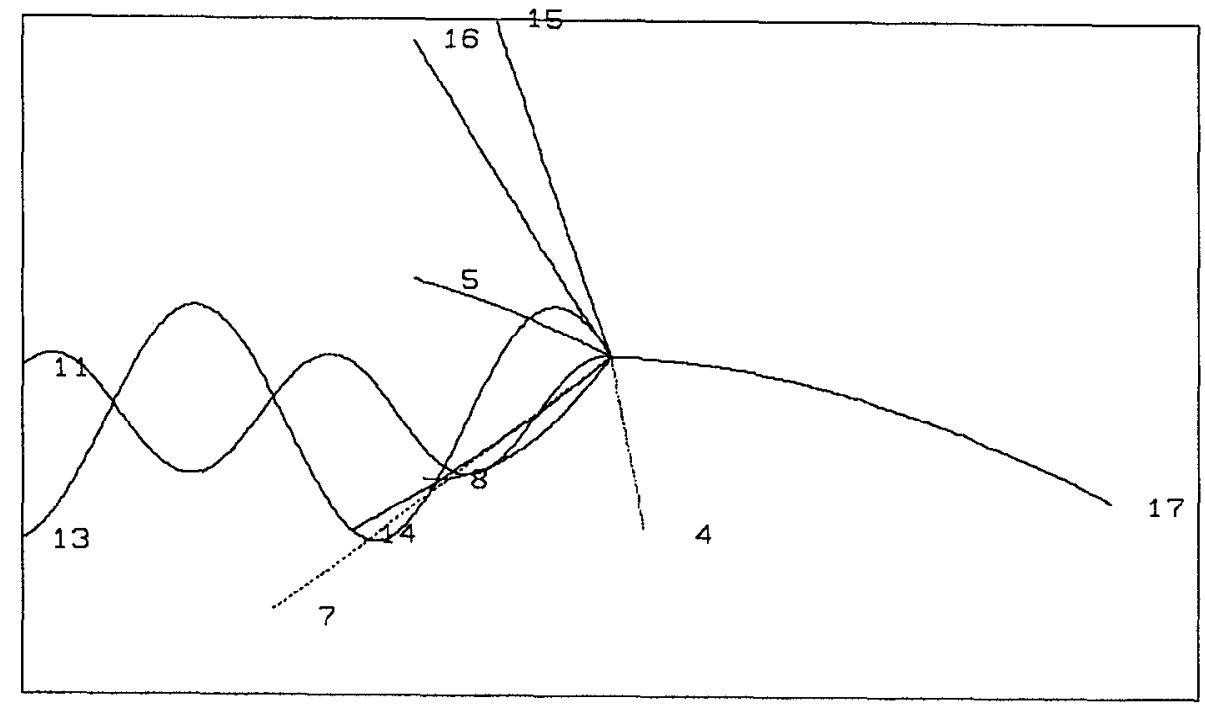

(b)

$$
\begin{array}{r}
\Upsilon(4 \mathrm{~S}) \rightarrow \overline{\mathrm{B}} \stackrel{\mathrm{B}}{\rightarrow} \nu_{7} \mu_{8}^{+} \stackrel{\mathrm{D}^{*-}}{\rightarrow} \pi_{13}^{-} \overline{\mathrm{D}}^{\circ}
\end{array}
$$

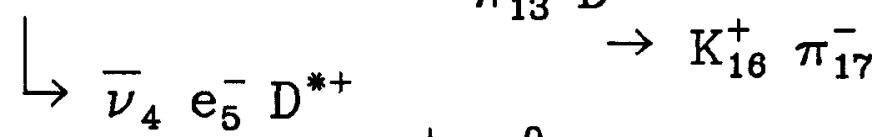

(c)

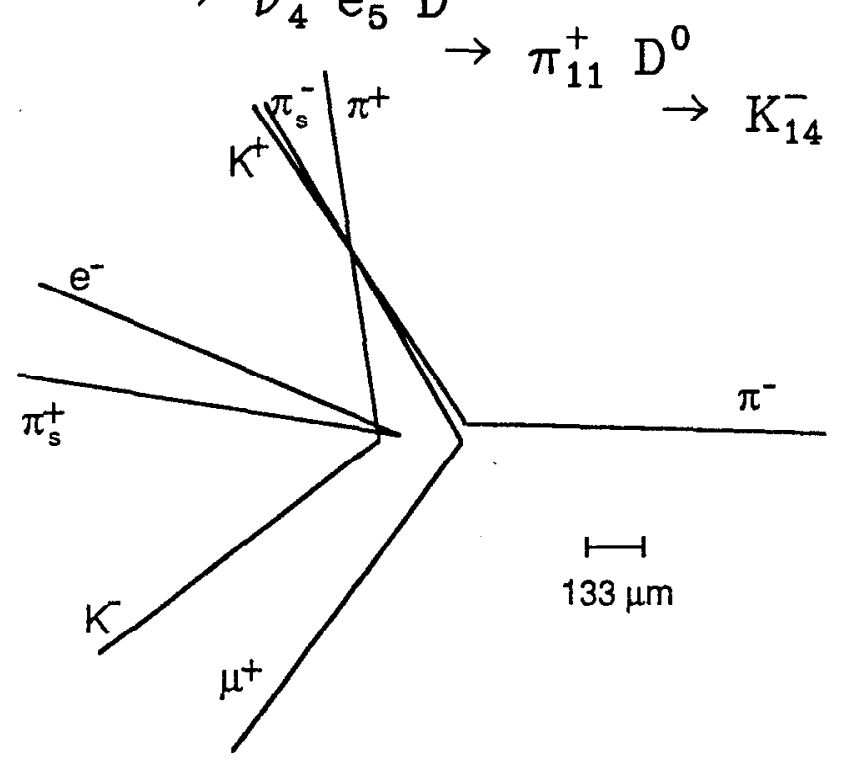

Figure 3.4 An example $B \bar{B}$ event. (a) All generated tracks, in $x z$ projection, framed by the inner dimensions of the electromagnetic calorimeter. (b) The decay chain; subscripts refer to the particle number in Figure (a). (c) A blowup of the vertices in the event; the two $B$ vertices are $133 \mu \mathrm{m}$ apart in $z$. 
Figure 3.5 The $d E / d x$ information available for the event in Figure 3.4. The track numbers now refer to detected tracks, rather than the generated tracks shown in that figure.

\subsection{Particle Identification}

Five different subsystems are available to provide charged particle identification. They are described briefly in the following sections. Note, however, that it may not be possible to include both TOF and CRID systems, due to space limitations and the degradation of low energy photon detection efficiency. To illustrate the information available from these systems, we refer to an example event shown in figures $3.4 \mathrm{a}-\mathrm{c}$.

$\mathrm{dE} / \mathrm{dx}$ in the Drift Chamber The drift chamber will provide up to 32 samples of $\mathrm{dE} / \mathrm{dx}$, each $1 \mathrm{~cm}$ long, in a gas mixture of $\mathrm{He}$ /isobutane $/ \mathrm{CO}_{2}$ at atmospheric pressure. We estimate a $\mathrm{dE} / \mathrm{dx}$.resolution of $7.5 \%$, and a ratio plateau/minimum of $\simeq 1.4$. A display of the $\mathrm{dE} / \mathrm{dx}$ information available from the event in Figure 3.4 is shown in Figure 3.5.

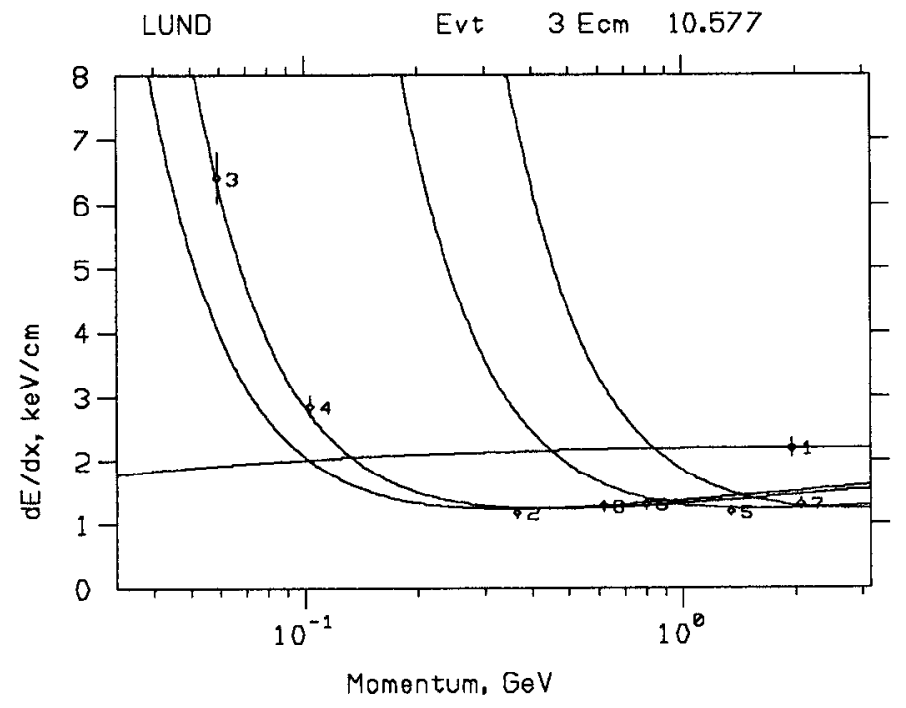

TOF A TOF system is available, with $5 \mathrm{~cm}$ scintillator in the barrel region from 80 to $85 \mathrm{~cm}$, and in the endcap regions from $150<|Z|<155 \mathrm{~cm}$. The nominal resolution is 75 psec, nominal efficiency is $100 \%$, and the system is assumed capable of resolving tracks independent of their angular separation. A display of the TOF information available from the event in Figure 3.4 is shown in Figure 3.6. 


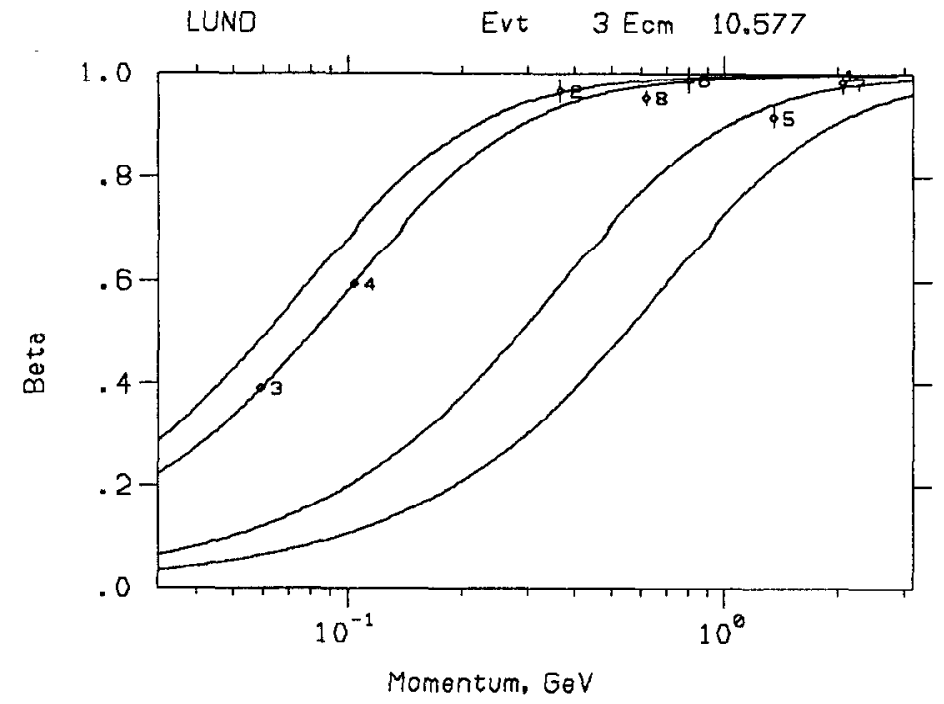

CRID A CRID system is available, based on $1 \mathrm{~cm}$ of $C_{6} F_{12}$ $(\mathrm{n}=1.277)$ radiator, proximity focussed over $10 \mathrm{~cm}$ onto a drift detector. The radiator provides $N_{0}=61$ photons, but total internal reflection is assumed to reduce this to 36.6. The CRID angle is measured with $100 \%$ efficiency over $|\cos \theta|<$ 0.95 , and the accuracy is degraded by contributions from measurement error in the drift detector, multiple scattering, track curving, chromatic error, and geometrical errors. A display of the CRID information available from the event in Figure 3.4 is shown in Figure 3.7.

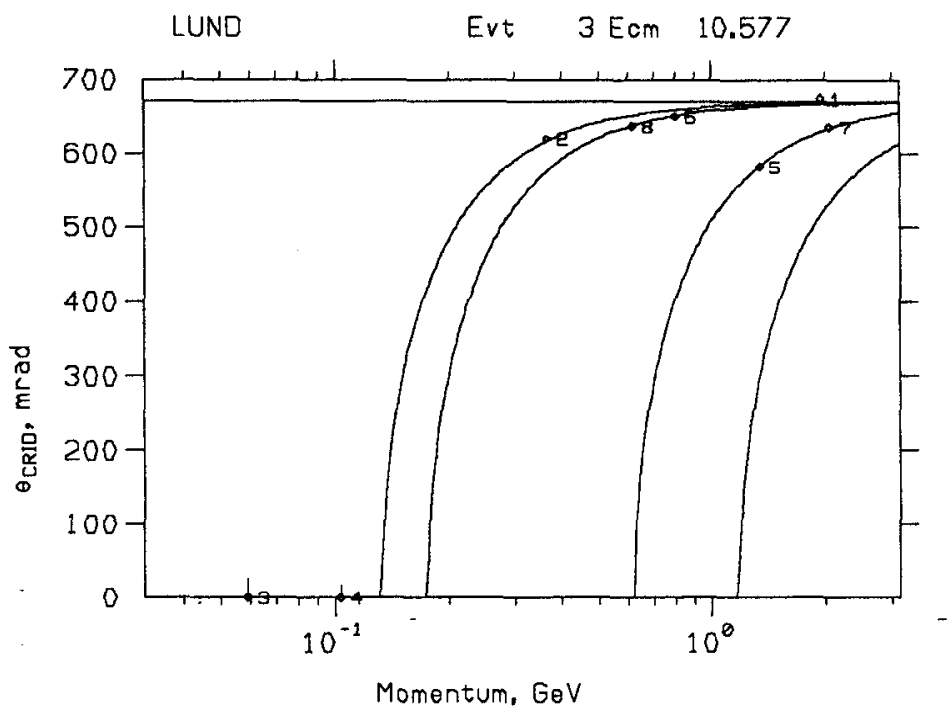

Figure 3.6 The TOF information available for the event in Figure 3.4. The track numbers now refer to detected tracks, rather than the generated tracks shown in that figure.
Figure $\quad 3.7 \quad$ The CRID information available for the event in Figure 3.4. The track numbers now refer to detected tracks, rather than the generated tracks shown in that figure. 
Figure 3.8 Pion misidentification probability in the muon rangeout counter, versus track momentum. e/ $\pi$ separation in Calorimeter Since it is notoriously difficult to simulate EM calorimeter response to hadrons at low energy (particularly with a fast MC), we have instead taken a simpler approach, which is to use a parameterization of the $\mathrm{e} / \pi$ separation (versus momentum) in an EM calorimeter. The efficiency for making a positive identification grows from $90 \%$ to $98 \%$ with increasing momentum. Electron misidentification probabilities fall from $5 \%$ to $1 \%$ with momentum, and pion misidentification falls from $5 \%$ to $0.5 \%$ with momentum.

$\pi / \mu$ separation in Muon Range-out Detector This is handled with a simple parameterization, just as with the e/ $\pi$ separation in the calorimeter. The efficiency for making a positive identification is taken to be $95 \%$ for tracks with $p>0.25$ $\mathrm{GeV} / \mathrm{c}$. Muon misidentification probabilities fall from $5 \%$ to $1 \%$ with momentum. Pion misidentification is complicated by decays and "punch-through". The misidentification probability assumed is shown in Figure 3.8.

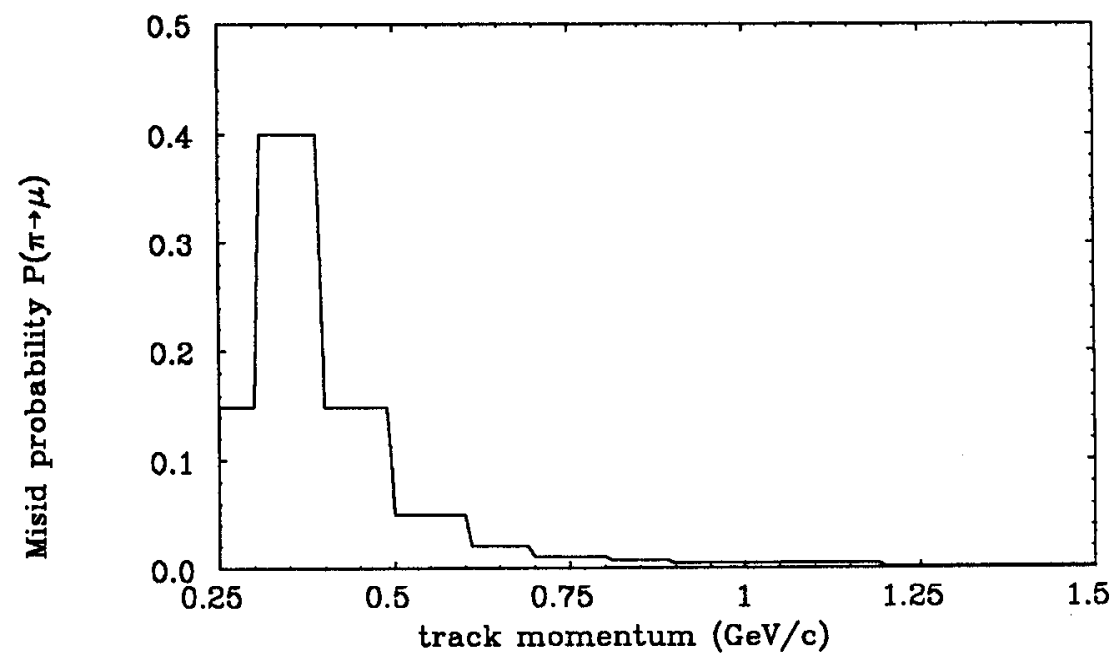




\subsection{VERTEXING}

The event generation assumes round beams of dimensions $50 \mu \mathrm{m} \times 50 \mu \mathrm{m} \times 1.5 \mathrm{~cm}$. The track impact parameters are smeared as described above. Software is available for finding the crossing point of track pairs and evaluating the track parameters there (for $K_{S}$ and $\Lambda$ finding). A software package is also available for fitting a common vertex to a specified set of tracks, and returning the position and error matrix for that vertex, as well as a $\chi^{2}$ for the fit.

\subsection{Analysis ToOLS}

Other analysis tools available to the user include VECSUB, a four-vector manipulation package (which interfaces to the Lund routines for doing sphericity, thrust, jet clustering, and Fox-Wolfram analyses). Handypak, Unified Graphics, a 3D event display, and particle ID event displays are also available.

The beam spot is assumed to be $50 \mu \mathrm{m} \times 50 \mu \mathrm{m} \times 1.5 \mathrm{~cm}$. 


\section{MASKING, BEAMPIPE AND VERTEXING ISSUES}

$\mathrm{T}$ The detector environment at a high-luminosity asymmetric $B$ factory is, in many ways, similar to that encountered by currently operating experiments in the $\Upsilon$ region. There are several aspects of the environment which are different. The first of these, the fact that the $\Upsilon$ system is moving in the laboratory, which is crucial to the measurement of CP violation, poses no particular problems. The detector modeled in Chapter 3 is, in the main, quite conventional, although the requirements of good particle identification and high-quality photon detection present interesting challenges. The goal of high-sensitivity studies of the photon transitions within the $\Upsilon$ family places requirements on the energy and angular resolution of the photon detector which can certainly be met (see Chapter 7). The rate of good physics events is such that a data acquisition system must be designed with far higher rate capability than has heretofore been the case in $e^{+} e^{-}$detectors. The situation is one which has been successfully met in numerous fixed target experiments; the necessary rate capability can be achieved in a straightforward manner. The most interesting challenges we will have to face occur in the immediate vicinity of the interaction region. The high currents, taken together with the vertexing requirements, present new combinations of design restrictions in the area of masking, beampipe heating and vertex detection.

\subsection{MASKING}

The masking of synchrotron radiation and off-energy electron induced backgrounds is very tightly coupled to the specific design of a machine lattice and final-focus system. This is a complex problem, which is discussed in some detail in the accompanying report $A$ Feasibility Study for an Asymmetric $B$ factory Based on PEP, and will not, therefore, be treated here. It should be mentioned that the masking problem is substantially more tractable in a crab-crossing scheme than in zero crossing angle optics. Preliminary studies indicate that practical masking schemes with beampipe radii which are quite acceptable for vertex finding can be found. 
The variation of vertex-finding precision with beampipe radius is discussed in Chapter 5, as is the correlated effect on the measurement of CP-violating asymmetries.

\subsection{Beampipe CoOling}

The heating of the beampipe by image currents and higherorder-mode losses is another concern. The combination of these two sources will represent a heat load of many $\mathrm{kW}$. Can this heat be removed without engendering an unacceptable temperature rise, while maintaining a sufficiently thin beampipe that multiple coulomb scattering does not compromise vertex detection precision? This question has led us to contract with the Jet Propulsion Laboratory in Pasadena for a conceptual design study of the cooling of a beampipe with a wall thickness which is the equivalent of $1 \mathrm{~mm}$ of beryllium. First indications are that the design criteria can indeed be met. There are, in fáct, several options, involving two-phase water cooling, single phase cooling with He gas, "perspiration cooling" with water droplets and the use of heat pipes. These options are being studied in detail; a single concept will shortly be chosen by JPL and an engineered design presented. See the accompanying accelerator feasibility study for a somewhat more detailed discussion

\subsection{VERTEX ISSUES}

The vertex detector is a critical element in the design of the experiment for the asymmetric $B$ factory. The vertex detector must meet the requirements imposed by the physics and the environment, if we are to realize the benefits of an asymmetric machine. Since no experiment operating in the $\Upsilon$ region has experience with such a device, we cannot extrapolate from present performance.

Fortunately, as the physics studies in this report clearly demonstrate, a vertex detector typical of those now in use is quite adequate for our purposes. The most interesting events for our purposes in the $\Upsilon$ region have tracks with typical momenta of $\sim 1 \mathrm{GeV} / \mathrm{c}$. With a beampipe having a wall thickness of 0.005 radiation lengths, including radiation shielding,
Conceptual designs of a beampipe with adequate cooling and low multiple-scattering contribution are under development 
The vertex resolution is dominated by multiple coulomb scattering

Requirements on vertex-finding are less stringent than those in existing fixed-target experiments and a typical first layer of silicon tracking, the resolution at the vertex is about $30 \mu \mathrm{m} / p(\mathrm{GeV} / \mathrm{c})$ due to multiple coulomb scattering alone for tracks at $90^{\circ}$. (The calculation placed the scattering material at an average radius of $2.3 \mathrm{~cm}$.) Since the resolution for tracks below $2-3 \mathrm{GeV} / \mathrm{c}$ is dominated by multiple scattering, and not by intrinsic spatial resolution, the actual spatial resolution of the vertex detector does not represent a stringent requirement.

Although this multiple-scattering-dominated resolution is insufficient to assign every track in the event to a unique vertex using only geometrical information, this assignment is not, in fact required. The requirements on the vertex detector are to measure the decay times of the $B$ mesons well enough to follow the time evolution of mixing and therefore $\mathrm{CP}$ violation in decays to CP eigenstates, and to reduce backgrounds by rcmoving some of the particle combinations from consideration when making mass spectra. These are much less demanding requirements than full geometrical vertex reconstruction, and certainly less demanding than the requirements of large background reduction posed by current fixed-target charm experiments.

The performance of the vertex detector in studying $\mathrm{CP}$ violation is spelled out in some detail in Chapler 5 for the decay $B^{o} \rightarrow J / \psi K_{s}^{o}$. The assignment of tracks to vertices is straightforward, since the $B^{0}$ decay is completely reconstructed without requiring vertex information. The $\bar{B}^{o}$ vertex is reconstructed using all of the other tracks in the event, knowing that the z-axis is constrained by the position of the $B^{o}$ decay. The role of the vertex detector is to measure $t-\bar{t}$, the time difference between the $B^{o}$ and $\bar{B}^{\circ}$ decays, which can be done with a resolution of better than one-half the $B$ lifetime. With this resolution, the measured asymmetry is only slightly worse than that seen in an ideal detector. Similarly, the vertex resolution is better than that needed to study $D^{o}-\bar{D}^{o}$ mixing, which is described in the charm physics section (Chapter 9). Some speculations on ways of achieving even better vertex-finding performance in the future are contained in an Appendix.

An important example of the background reduction from 
a vertex detector is the decay mode $B^{o} \rightarrow \pi^{+} \pi^{-}$, discussed in Chapter 6. With fairly simple cuts, which may not yet be optimized, the signal to background is improved by a factor of more than 4, with an efficiency of $62 \%$. Much of this gain is due to a global cut on vertex topology, which can be used in a general way to reduce continuum background for all $B$ physics, thus also reducing the amount of time needed for running below the $B \bar{B}$ threshold.

The other important feature for a vertex detector is its ability to tolerate the background level at the high luminosity $B$ factory. This is true no matter what detector technology is considered. Experience at CESR and DORIS, gained in recent tests aimed at understanding this environment, is quite valuable in this regard; we will therefore summarize these results here.

A feasible design for a vertex detector depends principally on what is a tolerable background rate. This seems to be true no matter what detector technology is considered, i.e., wire chamber or silicon-strip device, as naive estimates in both cases lead to roughly the same maximum tolerable rates. Pixel devices can tolerate a higher occupancy. The vertex resolution will, of course, hinge on this choice, but especially in view of the importance of multiple scattering, this may be a secondary consideration. Occupancy questions, as well as the practicalities associated with handling the heat load in the vertex chamber beampipe caused by higher-order mode losses and ohmic heating, may dominate the ultimate technical choice.

Three possible background sources need be considered in addressing this question: synchrotron photons, particle showers from coulomb scattered beam particles striking the beam pipe, and high-energy photons from bremsstrahlung of beam particles. Each of these processes produces ionization in the vertex detector, and ultimately a detected current from the apparatus. The latter determines operational lifetime, trigger rate and occupancy. Based on present experience, including small-diameter beam tests at CESR and DORIS, some conclusions can already be drawn, which allow for extrapolation to conditions at a $B$ factory.
Simple vertex cuts reduce the combinatoric background by a factor of 4 in an asymmetric machine 
Occupancy in the vertex detector is the primary consideration
The first of these is that the requirements of a reasonable occupancy per detector element will limit the tolerable background rate before the useful lifetime of the device is reached. Consider a hypothetical wire chamber, with anodes of $20 \mathrm{~cm}$ length. For such devices, the maximal amount of collected charge before serious degradation of performance varies from 0.1 to 1 Coulomb/cm, depending on gas composition. Therefore, a useful lifetime of 2 years translates into currents of between 0.4 and $4 \mu \mathrm{A}$ per wire. Typical beam crossing rates of $40 \mathrm{MHz}$ lead to occupancies per wire in excess of $100 \%$ for modest gas gains of $\sim 5 \times 10^{4}$. Clearly this is unacceptable; a $10 \%$ occupancy translates into a current draw of $0.03 \mu \mathrm{A} /$ wire. A similar calculation for silicon micro-strip detectors shows that there, too, occupancy is the critical issue, although questions of integration time and readout pitch are important assumptions in reaching this conclusion. Once again, it must be remembered that the new generation of pixel devices currently under development, can tolerate higher occupancy.

Background rates with small diameter beam pipes have been explored recently at CESR. With a $2.3 \mathrm{~cm}$ radius pipe of $1 \mathrm{~mm}$ aluminum, CLEO observed a current draw of $3 \mu \mathrm{A}$ in the innermost layer ( 64 cells) of their vertex chamber, and $10 \mu \mathrm{A}$ in total for single beam currents of $43 \mathrm{~mA}$. Assuming a gas gain of $5 \times 10^{4}$, these translate into 130 and 420 primary ion pairs per crossing respectively. The average hit multiplicity with a random trigger was found to be $8 /$ wire per beam crossing.

In explaining the observed current, all three sources of background noted above were considered. Fluorescent photons or synchrotron $\mathrm{X}$-rays above about $20 \mathrm{keV}$ could pass through the aluminum beam pipe. In argon-ethane, a $20 \mathrm{keV}$ photon would produce 660 ion pairs, so that the observed total current in the VDC is equivalent to $2 / 3$ of a photon per crossing. If synchrotron light were the dominant source of background, one would expect a small hit multiplicity, no dependence on the beam tube residual pressure and a strong energy dependence. None of these conditions was observed.

Beam particles which coulomb-scatter from residual gas 
molecules in the region between 4 and $8 \mathrm{~m}$ from the interaction point are spread out into a broad ribbon after passing through the final focus. Some fraction strike synchrotron radiation collimators or the beam pipe producing showers in the VDC. If a typical shower consists of 20 tracks, then one expects $3 \times 10^{4}$ ion pairs per incident electron. Monte Carlo calculations for a pressure of $10^{-8} \mathrm{~N}_{2}$ equivalent predict a scattered electron rate of $5 \mathrm{kHz}$, or an expected 50 ion pairs per crossing in the VDC. One expects to observe tracks in the chamber, a dependence on the pressure in the so called "coulomb region" and on the beam current. Although not many tracks were found, the latter two conditions were observed. It is possible that the Monte Carlo prediction of the rate is not reliable, since an understanding of the non-gaussian tails of the beam is required. Changes in pressure in the region from 10 to $50 \mathrm{~m}$ from the interaction failed to produce a strong change in the background rate, leading to the rejection of bremsstrahlung photons as a background source.

If the observed backgrounds in the CLEO vertex detector are assumed to be due to coulomb-scattered beam particles, as the bulk of the evidence indicates, then the product of beam current times pressure in the "coulomb region" of the beam tube can be used to scale to the $B$ factory. The innermost layer would draw about $20 \mu \mathrm{A}$ and have an occupancy of $7 \%$ for total beam currents of $1.5 \mathrm{~A}$, assuming that a residual pressure of $10^{-9}$ torr, which is feasible, can be achieved. However, if a third of the observed current in the present VDC is due to synchrotron photons, then this translates into a predicted current of $160 \mu \mathrm{A}$ in the innermost layer at a $B$ factory, which is impractical. Because of the large number of ion pairs produced in a chamber by a synchrotron hit, the flux of X-ray photons through the vertex region must be kept below about $10 \mathrm{MHz}$. A similar restriction can be derived for silicon microstrip detectors, where segmentation is finer, but integration times are longer.

The difficulties in combining the requirement of having minimum amount of material between the interaction point and the vertex detector with the requirement of a tolerable flux of synchrotron photons has been emphasized by a recent small-diameter pipe test at DORIS. There a $1.8 \mathrm{~cm}$ radius a vacuum of $10^{-9}$ torr in the interaction region is required 
Requirements on small radius and minimal material are less demanding at an asymmetric storage ring, since moderate vertex resolutionis sufficient pipe of $0.5 \mathrm{~mm}$ beryllium was inserted into the empty Crystal Ball interaction region and surrounded by 4 quadrants of wire chambers. Rescattered light from nearby copper synchrotron scrapers was visible in the form of a copper K-line in the pulse height spectra, and in fact dominated the observed counting rates. Projected counting rates on the innermost layers of the new ARGUS vertex drift chamber approach $150 \mathrm{kHz}$ at an operating pressure of $3 \mathrm{Atm}$ absolute. Better shielding will reduce this to tolerable levels for ARGUS, but the experience emphasizes the importance of synchrotron radiation shielding for $B$ factory applications.

The Monte Carlo studies presented in this report assume $1 \mathrm{~mm}$ beryllium equivalent of material, a part of which could be taken up by absorbers used to shield against rescattered synchrotron light. Moreover, since most of the primary photons are due to the high-energy beam, double masking will be possible. In addition, studies indicate that the nominal $2 \mathrm{~cm}$ radius assumed here could be increased to as much as $3 \mathrm{~cm}$ without serious degradation in the physics potential of the experiment. Although the requirement that no more than $\sim 10 \mathrm{MHz}$ of $\mathrm{X}$-ray photons be seen by the vertex detector is a challenge, calculations of suitable masking schemes for proposed machine designs are already within reach of this goal. 


\section{Measuring CP Violation AT AN ASYMMETRIC COLLIDER}

\subsection{INTRODUCTION}

$\mathrm{M}$ easurement of $\mathrm{CP}$ violation in the $B$ meson system provides the possibility of making sensitive consistency tests of the Standard Model. Measurements in the past few years of two properties of this system have revealed that the measurement of CP violation is more feasible than had previously been thought. The first is the measurement of the long lifetime of the $B$ mesons $^{1}$ ( $\tau_{B} \simeq 1.2 \mathrm{ps}$ ). This long lifetime, together with the recent development of precise vertex detection techniques, make the observation of detached $B$ vertices possible. Secondly, a high degree of mixing between $B^{0}$ and $\bar{B}^{0}$ has been reported by the $\mathrm{ARGUS}^{2}$ and $\mathrm{CLEO}^{3}$ collaborations. This introduces the exciting possibility of measuring $\mathrm{CP}$ violation in the $B$ system. This would be the first detection of CP violation outside the $K^{0}$ system.

We describe in this chapter a method for measuring CP violation at an asymmetric-energy $e^{+} e^{-}$collider which takes advantage of the long lifetime of the $B$ meson and depends on the $B \bar{B}$ mixing. If a $\mathrm{CP}$ eigenstate is detected in conjunction with an identified $B^{0}$ or $\bar{B}^{0}$, the distribution of the flight path between decays contains a term which provides a measurement of $\mathrm{CP}$ violation, as described below. The moving $B$ mesons in the laboratory make this flight path measurement possible. The use of the $\Upsilon(4 S)$ provides the largest $B \bar{B}$ cross section in $e^{+} e^{-}$production, other than from the $Z^{0}$, and allows excellent background rejection because the $B$ is monoenergetic in the center-of-mass frame. Since there are no extra fragmentation products accompanying the $B \bar{B}$ in the event, very simple tagging strategies can be used to separate $B^{0}$ from $\bar{B}^{0}$ decays, providing a tagging efficiency of $\approx 50 \%$. The 1988 Snowmass Summer Study group on $e^{+} e^{-} B$ factories concluded that this method required the least luminosity to measure $C P$ violation in the $B$ system, compared to any other $e^{+} e^{-}$technique, except for a polarized $Z$ factory. The CP eigenstates studied in detail here are $J / \psi K_{S}^{0}$, (for measuring $\sin 2 \beta$ ), $\pi^{+} \pi^{-}$(for measuring $\sin 2 \alpha$ ) and $\rho^{0} K_{S}^{0}$ from
The long $B$ lifetime and high degree of $B^{0} \bar{B}^{0}$ mixing mean that the experimental detection of $C P$ violation in the $B$ system has become more feasible than was originally predicted 
At the $\Upsilon(4 \mathrm{~S})$, a $B \bar{B}$ pair remains in a coherent state as long as neither has decayed.
$B_{s}$ decay (for measuring $\sin 2 \gamma$ ).

We discuss the advantages of an asymmetric energy $e^{+} e^{-}$ collider, the physics behind CP violation in the $B$ system, and our analysis methods. We then study the effects of different boosts and different beampipe radii on the measurement. We conclude with a comparison of possible measurements of $\mathrm{CP}$ violation at an asymmetric and symmetric $e^{+} e^{-}$collider.

Why an asymmetric energy collider?

Most measurements in the $b$ quark sector (with the exception of the $\Upsilon$ discovery itself) come from $e^{+} e^{-}$colliders operating in two energy regions which offer complementary advantages and disadvantages: (1) a center-of-mass energy equal to the $\Upsilon(4 \mathrm{~S})$ mass where the cross section is high and exclusive $B \bar{B}$ final states are produced with the $B$ mesons essentially at rest, or (2) a center-of-mass energy in the continuum where the cross section is lower and inclusive final states are produced, but the $B$ mesons are moving in the laboratory frame, making lifetime measurements possible.

In addition to the higher rate for producing $B \bar{B}$ exclusive states on the $\Upsilon(4 S)$ resonance, there are several other advantages. Since only two particles are produced, one can use the constraint that each $B$ meson energy is equal to the beam energy to reduce the combinatorial background when reconstructing final states. Another benefit of exclusive $B \bar{B}$ states is that having tagged one of the $B$ 's as a $B_{u}$ or $B_{d}$, one is guaranteed that the second $B$ is of the same type. Furthermore, there is a great advantage in having produced two spin-0 particles in a $p$-wave state. Because of Bose statistics, a $B^{0} \bar{B}^{0}$ pair will remain in a coherent $B^{0} \bar{B}^{0}$ or $B_{1} B_{2}$ state as long as neither $B$ has decayed. This coherence is extremely important for measuring mixing or $\mathrm{CP}$ violation. One of the most promising ways to observe $C P$ violation in the $b$ sector is to use the $B$ decays to $C P$ eigenstates $f_{C P}$ where $C P$ violation manifests itself in the interference of the amplitudes $A\left(B^{0} \rightarrow f_{C P}\right)$ and $A\left(B^{0} \rightarrow \bar{B}^{0} \rightarrow f_{C P}\right)$. In order to detcct this $\mathrm{CP}$ violation, one must know the nature of the particle $\left(B^{0}\right.$ or $\widetilde{B}^{0}$ ) at a given time. On the $\Upsilon(4 \mathrm{~S})$, tagging one $B$ as a $B^{0}$ or a $\bar{B}^{0}$ identifies the other with certainty. 
Because, as we will show below, the CP-violating asymmetry is an odd-function of the time difference between the $B$ meson decays (when the $B$ 's are in a $p$-wave), integrating over all times will yield no asymmetry. Thus one must also measure the time order of the decays to be sensitive to $C P$ violation. Since the $B$ mesons are produced almost at rest at a symmetric machine - the $B$ momentum is about $330 \mathrm{MeV}$, and the average decay length of the $B$ 's is about $20 \mu \mathrm{m}-$ a measurement of the $B$ lifetimes and, consequently, of any time dependent effects, is impossible with present vertex detection technology. In spite of the advantage of having the center-of-mass energy equal to the mass of the $\Upsilon(4 \mathrm{~S})$, it is currently not practical (with a symmetric machine) to study the time dependence of the $B$ decays, and therefore it is not possible to measure CP violation using the $B^{0} \bar{B}^{0}$ mixing scenario outlined above.

The conventional method, in $e^{+} e^{-}$annihilation, of producing $B$ mesons moving in the laboratory frame is to operate at a center-of-mass energy in the continuum above the $\Upsilon$ resonances. At a center-of-mass energy of $15 \mathrm{GeV}$, the average decay length of the $B$ mesons is about $300 \mu \mathrm{m}$, a decay length which can be measured with present technologies. Unfortunately, there are also disadvantages in this energy region.

First, the cross section is lower by a factor of eight at a center-of-mass energy of $15 \mathrm{GeV}$ compared with the $\Upsilon(4 \mathrm{~S})$. Since the $B$ mesons are no longer produced with the beam energy, one must fully reconstruct a $B$ in order to determine its momentum which is required to study the time dependence. Furthermore, mixing and $\mathrm{CP}$ violation studies are more complicated, since the quantum coherence of the initial state is lost and each $B$ meson state can evolve independently of the other $B$ state. This means that tagging the second $B$ does not guarantee that one has determined the real nature of the first one at the time of production. For example, a high energy positron might tag a $B^{+}$, a $B^{0}$, or a $\bar{B}^{0}$ which has mixed to a $B^{0}$. The effect of the $B_{s}^{0} \bar{B}_{s}^{0}$ and $B_{d}^{0} \bar{B}_{d}^{0}$ mixing is to produce an apparent dilution of the $C P$ violation asymmetry. This dilution (from tagging confusion) requires a factor of two more events to make a measurement of the same statistical signif-
At a symmetric $e^{+} e^{-}$collider, $B$ mesons produced at the $\Upsilon(4 \mathrm{~S})$ have a decay length too short to measure.

The lack of quantum coherence above the $\Upsilon(4 \mathrm{~S})$ complicates the measurement of $C P$ violation and produces an additional dilution factor. 
An asymmetric collider allows both exclusive $B \bar{B}$ production with a large cross section and lifetime measurements.
The first $B$ decay projects the other $B$ into a known (pure) quantum state. Hence, it is the time difference between decays which is relevant to the measurement of $C P$ violation. icance. Together with the lower cross section, one suffers so greatly in terms of rate that the advantage of having moving $B$ 's in the laboratory frame is lost.

A solution that overcomes the disadvantages of these two schemes while retaining their positive aspects is to produce the $\Upsilon$ resonances moving in the laboratory frame, which can be achieved by colliding two beams of unequal energy. ${ }^{6}$ This results in two $B$ 's boosted in the same direction along the beam axis. The average distance between the two $B$ decays is approximately $\beta \gamma c \tau$ where $\beta$ and $\gamma$ are the boost parameters of the center of mass and $\tau$ is the average $B$ lifetime. This method allows both the production of exclusive $B$ meson states with a relatively large cross-section and the measurement of the lifetimes that enable the detection of a violation of CP symmetry.

\subsection{Using CP Eigenstates at the $\Upsilon(4 \mathrm{~S})$ TO MEA- SURE CP VIOLATION}

\section{Formalism}

The $\Upsilon(4 \mathrm{~S})$ decays into $B^{0} \bar{B}^{0}$ in a p-wave state which imposes special correlations on the final state. The unique initial state implies that if the first neutral $B$ decays into a final state $f_{1}$ at time $t_{1}$, this projects the remaining neutral $B$ onto the orthogonal state which cannot decay into $f_{1}$. This state then propagates in time and we observe its decay at time $t_{2}$ into final state $f_{2}$. We can think of the first decay as preparing the second $B$ in a special state at $t_{1}$, whose propagation then depends only on the time difference between decays $t_{2}-t_{1}$.

The final states $f_{1}$ or $f_{2}$ that are observed can either be unique to $B^{0}$ or $\bar{B}^{0}$ only (for example, the primary decay into a lepton of a given charge) or can receive contributions from both types of $B$. In the case of a CP eigenstate for $f_{1}$ or $f_{2}$, the contributions from each $B$ are essentially equal in magnitude. A decay unique to $B^{0}$ or $\bar{B}^{0}$ is said to tag the $B$ flavor. We can measure mixing in the $B$ system if both $f_{1}$ and $f_{2}$ are tagging decays. CP violation can be measured, in the presence of mixing, if one decay is a tagging decay and the other is a CP eigenstate. The observation of an event in 
which both $B$ mesons decayed into eigenstates of the same $\mathrm{CP}$ would also indicate $\mathrm{CP}$ violation, but the rates in this case are too small to be useful.

The decay to a tagging state plus a $\mathrm{CP}$ eigenstate provides four configurations which have to be separately analyzed as a function of $t_{2}-t_{1}$ in order to measure $C P$ violation. For the possible final states which provide a $B^{0} \operatorname{tag}\left(f_{B}\right)$, a $\bar{B}^{0}$ $\operatorname{tag}\left(f_{\bar{B}}\right)$ or a CP eigenstate $\left(f_{\mathrm{CP}}\right)$, the choices of decays and associated times are:

(1) $f_{B}\left(t_{1}\right) f_{\mathrm{CP}}\left(t_{2}\right)$

(2) $f_{\mathrm{CP}}\left(t_{1}\right) f_{B}\left(t_{2}\right)$,

(3) $f_{\bar{B}}\left(t_{1}\right) f_{\mathrm{CP}}\left(t_{2}\right)$

(4) $f_{\mathrm{CP}}\left(t_{1}\right) f_{\bar{B}}\left(t_{2}\right)$.

$\mathrm{CP}$ violation produces a distribution in $t_{2}-t_{1}$ which is different for (1) and (4) from that for (2) and (3). Summing over (1) and (2) or (3) and (4), as would be done if no vertex information were available, removes the $\mathrm{CP}$-violating asymmetry. A measure of $\mathrm{CP}$ violation is therefore given by the asymmetry in rates

$$
A=\frac{(2)+(3)-(1)-(4)}{(1)+(4)+(2)+(3)}
$$

where each term is integrated over the same positive time interval, $t_{2}-t_{1}$. A change of sign of the $\mathrm{CP}$ of the state is equivalent to exchanging $B$ and $\bar{B}$ and therefore changes the sign of $A$.

We sketch below, to a very good approximation, the calculation of the rates for (1) and (2), as expected for a CP-even state. The mass eigenstates of definite lifetime for the $B$ system are

$$
B_{1}^{0}=\frac{e^{i \phi_{1}} B^{0}+e^{-i \phi_{1}} \bar{B}^{0}}{\sqrt{2}}, \quad B_{2}^{0}=\frac{e^{i \phi_{1}} B^{0}-e^{-i \phi_{1}} \bar{B}^{0}}{\sqrt{2}},
$$

giving

$$
B^{0}=\left(\frac{B_{1}^{0}+B_{2}^{0}}{\sqrt{2}}\right) e^{-i \phi_{1}}, \quad \bar{B}^{0}=\frac{\left(B_{1}^{0}-B_{2}^{0}\right)}{\sqrt{2}} e^{i \phi_{1}} .
$$

With no vertex information, no asymmetry can be measured using $C P$ eigenstates at the $\Upsilon(4 \mathrm{~S})$. 
The phase that is measured is independent of K-M matrix convention.
We define

$$
\begin{aligned}
m & \equiv \frac{m_{1}+m_{2}}{2}, \quad \Gamma \equiv \frac{\Gamma_{1}+\Gamma_{2}}{2} \\
\Delta m & \equiv m_{2}-m_{1} \quad \text { and } \Delta \Gamma \equiv \Gamma_{2}-\Gamma_{1} .
\end{aligned}
$$

Under reasonable assumptions in the Standard Model, it is expected that $\Delta \Gamma \ll \Gamma$ and $\Delta \Gamma \ll \Delta m$. Under these assumptions, the time evolution of $B_{1}^{0}$ and $B_{2}^{0}$ is given by

$$
\begin{aligned}
& B_{1}^{0}(t)=B_{1}^{0} e^{-(\Gamma t / 2+i m t)} e^{i \Delta m t / 2} \\
& B_{2}^{0}(t)=B_{2}^{0} e^{-(\Gamma t / 2+i m t)} e^{-i \Delta m t / 2}
\end{aligned}
$$

We take for the decay amplitudes

$$
\begin{aligned}
\left\langle f_{B}\left|H_{w}\right| B^{0}\right\rangle & =\mathcal{B}, \quad\left\langle f_{B}\left|H_{w}\right| \bar{B}^{0}\right\rangle=0 \\
\left\langle f_{\mathrm{CP}}\left|H_{w}\right| B^{0}\right\rangle & =\mathcal{A} e^{i \phi_{2}}, \quad\left\langle f_{\mathrm{CP}}\left|H_{w}\right| \bar{B}^{0}\right\rangle=\mathcal{A}^{\prime} e^{-i \phi_{2}} .
\end{aligned}
$$

where $H_{w}$ is the weak decay Hamiltonian, and $\phi_{2}$ is the phase from the Kobayashi-Maskawa $(\mathrm{K}-\mathrm{M})$ matrix. In the following we shall let $\mathcal{A}=\mathcal{A}^{\prime}$, which is a safe approximation.

The phases in the individual formulae shown above depend on the phase convention for the $\mathrm{K}-\mathrm{M}$ matrix. However, the final result below contains one phase which is a measurable invariant of the matrix and is independent of conventions. 10

For process (1), the state projected at $t_{1}$ is $\bar{B}^{0}$. With $t \equiv t_{2}-t_{1}$, the rate to subsequently detect a final state $f_{\mathrm{CP}}$ is proportional to

$$
\left|\left\langle f_{\mathrm{CP}}\left|H_{w}\right| \bar{B}^{0}(t)\right\rangle\right|^{2} \text {. }
$$

For process (2), the state projected at $t_{1}$ is

$$
\frac{B^{0} e^{-i \phi_{2}}-\bar{B}^{0} e^{i \phi_{2}}}{\sqrt{2}}
$$

and therefore the rate to subsequently detect $f_{B}$ is proportional to

$$
\left|\left\langle f_{B}\left|H_{w}\right|\left(B^{0}(t) e^{-i \phi_{2}}-\bar{B}^{0}(t) e^{i \phi_{2}}\right) / \sqrt{2}\right\rangle\right|^{2} .
$$

Inserting the time evolution of $B^{0}(t)$ and $\bar{B}^{0}(t)$, through their dependence on $B_{1}^{0}(t)$ and $B_{2}^{0}(t)$, and the decay amplitudes, 
we get the following expressions for the reaction rates:

$$
\begin{aligned}
& f_{B}\left(t_{1}\right) f_{\mathrm{CP}}\left(t_{2}\right) \propto|\mathcal{A}|^{2}|\mathcal{B}|^{2}[1-\sin (2 \phi) \sin (\Delta m t)] e^{-\Gamma t} \\
& f_{\mathrm{CP}}\left(t_{1}\right) f_{B}\left(t_{2}\right) \propto|\mathcal{A}|^{2}|\mathcal{B}|^{2}[1+\sin (2 \phi) \sin (\Delta m t)] e^{-\Gamma \iota}
\end{aligned}
$$

where $\phi=\phi_{1}+\phi_{2}$. The CP-violating asymmetry depends on the phase $\phi$, which in principle can be calculated from the $\mathrm{K}-\mathrm{M}$ matrix, and the presence of mixing which depends on the magnitude of $\Delta m / \Gamma$.

Note that if the Standard Model is not a complete description of weak interactions, observing CP violation in only one channel (e.g., $J / \psi K_{S}^{0}$ ) does not allow us to determine whether the $C P$ violation originates in the mixing $(\Delta B=2)$ or in the decay $(\Delta B=1)$. It is therefore necessary to observe $C P$ violation with sufficient accuracy in more than one decay mode in order to distinguish between different models.

Measurement for a moving $\Upsilon(4 S)$ system

For a moving $\Upsilon(4 S)$ system, where the momentum of the $B$ in the $\Upsilon(4 \mathrm{~S})$ rest frame can be ignored (this approximation will be discussed below), the distribution in $t$ translates directly into the distribution between the $B$ decay vertex positions along the flight direction of the $B$ mesons. For this study, with beam energies of 9.0 and $3.1 \mathrm{GeV}$, the boost is given by $\beta \gamma=0.56$. The distance between the $B$ decays within an event is $\Delta z \simeq \beta \gamma c t$, and therefore the mean $\Delta z$ has a value of $\beta \gamma c \tau \simeq 180 \mu \mathrm{m}$. The point of creation of the $\Upsilon(4 \mathrm{~S})$, which is not precisely measurable, is fortunately not needed for the analysis.

The exact relationship between $t$ and $\Delta z$, in terms of the velocity $\beta^{c m}$, the Lorentz factor $\gamma^{c m}$ and the polar angle $\theta^{c m}$ of the $B$ in the event's center of mass frame is

$$
\Delta z=c \beta \gamma \gamma^{c m} t+c \gamma \beta^{c m} \gamma^{c m} \cos \theta^{c m}\left(2 t_{1}+t\right)
$$

Reconstructing the momentum of one of the $B$ 's does not allow exact evaluation of $t$ since $t_{1}$ is unknown. Fortunately, since $\beta^{c m}$ is only about $0.06, \gamma^{c m}$ is very near $1(\approx 1.002)$, and the polar angle distribution of the $B$ meson in the centerof-mass system is peaked at large angles, the average error on
The distance between $B$ decay vertices along the boost direction is very nearly proportional to the time difference between decays. 
$t$ due to the above approximation is only on the order of $13 \%$ for $\beta \gamma=0.56$.

The CP-violating, time-dependent asymmetry defined earlier is simply given by

$$
A(t)=\sin 2 \phi \sin (\Delta m t) .
$$

This asymmetry, which depends on the time ordering of the decays, can be integrated $t$ over the measured time difference between decays. The result of integrating from some minimum time $t_{0}$ (to exclude events in which the time order of the decays was determined incorrectly) to $t=\infty$ is

$$
\begin{aligned}
A & =\left[\frac{\int_{t_{0}}^{\infty} \sin (\Delta m t) e^{-\Gamma t} d t}{\int_{t_{0}}^{\infty} e^{-\Gamma t} d t}\right] \sin 2 \phi \\
& =\frac{\left[\sin \left(\Delta m \tau_{0} / \Gamma\right)+(\Delta m / \Gamma) \cos \left(\Delta m \tau_{0} / \Gamma\right)\right]}{1+(\Delta m / \Gamma)^{2}} \sin 2 \phi,
\end{aligned}
$$

where $\tau_{0}=t_{0} \Gamma$. If all events are used, $\tau_{0}=0$, and

$$
A=\frac{(\Delta m / \Gamma)}{1+(\Delta m / \Gamma)^{2}} \sin 2 \phi=\frac{x}{1+x^{2}} \sin 2 \phi
$$

where $x \equiv \Delta m / \Gamma$ is the mixing parameter. The factor $x /(1+$ $\left.x^{2}\right)$, which dilutes the time-integrated, time-ordered asymmetry is roughly 0.5 for the $B_{d}$ system and smaller $\left(\approx 1 / x_{s}\right)$ for the $B_{s}$ system in which $x_{s}$ is expected to be large ( 3 or greater).

If $N$ detected events are available in total, then the error on the asymmetry is given by $\delta A=\sqrt{\left(1-A^{2}\right) / N}$. In order to make a three standard deviation detection of the $\mathrm{CP}$ violation, wc will need $N \simeq 9\left(1-A^{2}\right) / A^{2}$ events. Since $A$ is expected to be $\sim 0.05$ to 0.3 in the Standard Model, ${ }^{11}$ this means we need between 100 and 3600 events.

A cut requiring a non-zero $\tau_{0}$ would increase $A$ at the cost of reducing $N$. For $\Delta m / \Gamma \simeq 0.75$, a cut requiring $\tau_{0} \gtrsim 0.6$ increases the significance in terms of the number of standard deviations, $\delta A / A$, by about $20 \%$. 
Finally, it is important to note that the measurement allows several internal checks since the asymmetry changes sign if we exchange $B^{0}$ and $\bar{B}^{0}$, the CP of the final state, or the time ordering of the two $B$ decays.

\subsection{Physics and Detector Simulation}

In order for our new, expanded studies of CP violation using $B \rightarrow J / \psi K_{S_{5}}^{0}$ to be directly comparable to the previously published study ${ }^{5}$ we have continued to use the Monte Carlo simulation written for that paper to study this reaction. Our studies of $B \rightarrow \pi^{+} \pi^{-}$and $B_{s} \rightarrow \rho^{0} K_{S}^{0}$ utilized the simulation described in Chapter 3. We therefore include here a brief description of the $B \rightarrow J / \psi K_{S}^{0}$ simulation. This simulation includes a four-vector generator (written by Roy Aleksan) interfaced to the GEAN' ${ }^{12}$ detector simulation package.

\section{$\underline{B \text { Production and Decay }}$}

This program generates $B^{0} \bar{B}^{0}$ final states for a moving center of mass. In the center-of-mass frame, the $B$ 's are generated with a $\sin ^{2} \theta$ distribution with respect to the beam direction. The $B$ mesons decay either semileptonically or purely hadronically. The semileptonic branching ratios are set to $12 \%$ each for $e$ and $\mu$ and $2 \%$ for $\tau$. These decays come from the coupling of the $W$ to the $b$ quark and therefore exhibit the standard momentum distribution for $V-A$ coupling. The remaining spectator quark is then combined with the $c$ quark to form a $D$ or $\dot{D}^{*}$ meson with probabilities of $30 \%$ and $70 \%$, respectively.

For the purely hadronic decays we use a simple model, consistent with what is known about $B$ meson decay. The total multiplicity of primary decay mesons is selected according to a gaussian distribution with mean 4.7 and variance 3.2 . The decay multiplicity is bounded between two and ten. The ratio of pseudoscalar to vector mesons is chosen to be 1:2. The hadronic decay products are distributed according to a phase space angular distribution in the $B$ rest frame.

We feel that for this study, great accuracy in the hadronic decays of the $B$ is not necessary. The semileptonic $B$ decays and the charmed meson decays are more important, since
This method of using $C P$ eigenstates at the $\Upsilon(4 \mathrm{~S})$ allows important internal checks by comparing the four different reaction rates. 
The coherence of the $B^{0}$ and $\bar{B}^{0}$ mesons at the $\Upsilon(4 \mathrm{~S})$ is crucial to the measurement. these are the decays used for tagging $B$ flavor. Fortunately, these are relatively well understood and therefore are accurately simulated by the generator.

Once the $B$ decay is simulated, all unstable mesons decay according the branching ratios tabulated by the Particle Data Group $^{13}$ for these secondary particles. The $D^{+}, D^{-}, D^{0}$ and $\bar{D}^{0}$ mesons are exceptions; we use the most recently measured branching fractions. ${ }^{14}$ Twenty percent of charged $D$ decays and $10 \%$ of neutral $D$ decays have not been measured. For these decays, we produce final states with at least two neutral pions. The $D_{s}$ decays are simulated using the same method as for the $B$ meson. We have checked that the generator reproduces the measured decay modes ${ }^{14}$ reasonably well.

Since the measurement of the distance between the primary $B$ decays is a critical part of the $\mathrm{CP}$ violation measurement described here, the generator must simulate the flight distance of all longlived particles such as $B$ and $D$ mesons. These lifetimes have been set to the following values: $\tau\left(D^{0}\right)=0.44 \mathrm{ps}, \tau\left(D^{+}\right)=1.10 \mathrm{ps}, \tau\left(D_{s}^{+}\right)=0.40 \mathrm{ps}$, $\tau\left(B^{+}\right)=1.10 \mathrm{ps}$ and $\tau\left(B^{0}\right)=1.10 \mathrm{ps}$.

Mixing

The generator allows for $B_{d}^{0} \bar{B}_{d}^{0}$ mixing. At the $\Upsilon(4 S)$, both $B^{0}$ and $\bar{B}^{0}$ - or equivalently $B_{1}$ and $B_{2}$ - are in a coherent state, until one of the $B$ 's decays. This coherence is vital to the measurement as it means that the relevant starting time for time-dependence studies is the time at which the first $B$ decays and not the time at which both $B$ 's were produced. This is fortunate since the experimentally measurable quantity is the distance between $B$ decays if this distance is sufficiently large. We cannot measure the distance the $B$ 's travelled together before the first one decayed since the beam size is much larger in this dircction than the $B$ decay length. In the study described here, we assume the beam size is 400 $\mu \mathrm{m}$ in the horizontal transverse dimension, $40 \mu \mathrm{m}$ in the vertical transverse dimension and $2 \mathrm{~cm}$ in $z$ (again, purely for consistency with ref. 5).

The decay of the first $B$ (at time $t_{1}$ ) fixes the nature of the second $B$. The time dependence for the mixing between $B^{0}$ 
and $\bar{B}^{0}$ can be seen in the probability to observe a $B$ meson decay as a $B^{0}$ or $\bar{B}^{0}$ at time $t_{2}$ if it was a $B^{0}$ at time $t_{1}$ :

$$
P\left(B^{0}\left(t_{1}\right) \rightarrow \bar{B}^{0}\left(t_{2}\right)\right) \propto \frac{e^{-\Gamma\left(t_{2}-t_{1}\right)}\left[1-\cos \left(x \Gamma\left(t_{2}-t_{1}\right)\right)\right]}{2}
$$

and

$$
P\left(B^{0}\left(t_{1}\right) \rightarrow B^{0}\left(t_{2}\right)\right) \propto \frac{e^{-\Gamma\left(t_{2}-t_{1}\right)}\left[1+\cos \left(x \Gamma\left(t_{2}-t_{1}\right)\right)\right]}{2}
$$

where $x \equiv \frac{\Delta m}{\Gamma}$ represents the amount of mixing and has been measured by the ARGUS collaboration ${ }^{2}$ to be $0.73_{-0.18}^{+0.17}$ (and subsequently by the CLEO collaboration to be $0.68_{-0.20}^{+0.19}$ ) for the $B_{d}^{0} \bar{B}_{d}^{0}$ system. For this analysis it has been set to 0.75 .

\section{Detector Simulation}

Because an important part of this analysis is the measurement of the $B$ vertices and their relative location, accurate simulation of the multiple scattering of particles in matter is essential. We use the GEANT ${ }^{12}$ detector simulation package since, in addition to the multiple scattering, this package simulates all the other interactions or reactions that degrade the measurements such as bremsstrahlung, nuclear interactions, pair production, Compton scattering and decays of very longlived particles such as kaons or charged pions. The penalty for using this package is the large amount of computing time that is needed to simulate one event. Therefore, we only simulate the vertex detector environment in detail. We have simulated the central detector and calorimeter by simply smearing the momentum of a charged particle and the energy and angles of a neutral particle according to normal distributions with the following standard deviations:

$$
\frac{\sigma_{p_{T}}}{p_{T}}=0.005 \sqrt{1+p_{T}^{2}}, \quad \frac{\sigma_{E}}{E}=\frac{0.02}{\sqrt[4]{E}}, \quad \sigma_{\theta}=0.02 \text { radian } .
$$

In the above formulae, $p_{T}$ and $E$ are measured in units of $\mathrm{GeV}$. The angular resolution for the charged particles is then mainly due to the multiple scattering and the vertex detector resolution. Again, these distributions were used for consistency with ref. 5 .
This Monte Carlo study includes accurate simulation of multiple scattering and nuclear interactions in the beampipe and vertex detector. 
Since the two $B$ vertices are close in the $x-y$ plane, it is possible to idenitfy the primary vertex of one $B$ decay by matching its $x$ and $y$ coordinates with those of the other $B$ which has been reconstructed kinematically.
The important points for obtaining good vertex reconstruction are the amount of material before the first two measurements, the distance between the interaction point and the first measurement, and the vertex detector resolution. We simulate a $1 \mathrm{~mm}$ thick beryllium beam pipe at a radius of $10 \mathrm{~mm}$ (or $20 \mathrm{~mm}$ for some comparisons; see section 5.7) with two layers of silicon pixel detectors. The silicon thickness has been set to $300 \mu \mathrm{m}$; the two layers are located at radii of 12 $\mathrm{mm}$ and $42 \mathrm{~mm}$ (for the smaller beam pipe), or $22 \mathrm{~mm}$ and $52 \mathrm{~mm}$ (for the larger beam pipe) and extend $300 \mathrm{~mm}$ in both the forward and backward directions. The pixel resolution is set to $10 \mu \mathrm{m}$.

For a boost with $\beta \gamma=0.56$, particles emitted at a relatively large angle in the center-of-mass frame $\left(\left|\cos \theta_{c m}\right| \gtrsim 0.7\right)$, will have their angle reduced by a factor of roughly 0.6 . The momentum of most of the particles will be increased slightly comparcd to a symmetric machine, particularly for particles emitted at small forward angles. This is fortunate since these particles will cross more material than the ones emitted at large angles.

\section{Vertex Reconstruction}

The algorithm used to reconstruct primary $B$ and $D$ decay vertices is based on the detector model discussed above and assumes that the position errors at the vertex location are dominated by the resolution of the vertex detector and the multiple scattering in the beampipe and vertex detector.

The algorithm begins by extrapolating each track back to its distance of closest approach to the $z$ axis which is centered on the beam-beam collision region and points along the direction of motion of the $\Upsilon(4 \mathrm{~S})$ system. $B$ and $D$ meson vertices are expected to bc separated by less than a few hundred microns in the plane perpendicular to this axis and to lie in a few centimeter long region along this axis. In addition, the two primary $B$ decays are expected to be very close to each other in the transverse plane (i.e.to have the same $x$ and $y$ coordinates but different $z$ coordinates) since they have very little transverse momentum and therefore nearly follow the trajectory of the parent $\Upsilon(4 S)$ in the laboratory. Note that this is a very important constraint since it allows one to iden- 
tify a primary $B$ vertex without having to fully reconstruct $B$ or $D$ mesons, by matching its $x, y$ coordinates with those of a kinematically reconstructed $B$ meson.

The track position errors and particle lifetimes are such that in only a small fraction of the events are all vertices found with all particles correctly assigned. Therefore, the vertex finding is most useful when combined with external particle identification and kinematic information. In the physics discussion below, one $B$ decay will in fact be fully reconstructed using information mostly external to the vertex detector. The vertex information is then crucial for measuring the position of the second $B$ meson. We summarize the quality of the vertex information for this case (with $\beta \gamma=0.56$ ):

$56 \%$ of $B$ decays with only one vertex are found

$37 \%$ of $B$ decays with two vertices are found

$8 \%$ of $B$ decays with three or more vertices are found

The $z$ position error for the chosen primary $B$ vertex is $46 \mu \mathrm{m}$

The transverse position error for the chosen primary $B$ vertex is $36 \mu \mathrm{m}$.

\subsection{Measuring $\sin 2 \beta$ with $B \rightarrow J / \psi K_{S}^{0}$}

In order to measure CP violation, we must identify events in which one $B$ meson decays to a $\mathrm{CP}$ eigenstate (such as $\left.J / \psi K_{S}^{0}\right)$ and the other decays to a final state which identifies the $B$ meson as a $B^{0}$ or $\bar{B}^{0}$. The latter $B$ will be referred to as the tagging $B$. The relative decay position of the two $B$ 's must be measured to observe a CP-violating effect. In this section we explain in detail our methods, using the determination of $\sin 2 \beta$ as an example.

In this analysis, we will concentrate on the CP eigenstate $J / \psi K_{S}^{0}$ with the $J / \psi$ decaying to a lepton pair. Then the vertex position of the lepton pair gives the decay position of the $B$ decaying to a CP eigenstate. Since the $B$ mesons are produced almost at rest in the $\Upsilon(4 \mathrm{~S})$ system, the two $B$ 's will have almost the same decay position in the plane
The vertex finding is most effective when used in conjunction with additional kinematic information. 
The difference in decay position between $J / \psi$ and the primary vertex for the tagging $B$ measures $t_{2}-t_{1}$.

Figure 5.1. Example of a $B^{0} \bar{B}^{0}$ decay illustrating the fact that the two $B$ decay vertices are typically very close together in the plane perpendicular to the beam axis but separated in the direction along the beam axis.

Charged kaons and leptons are used to identify the tagging $B$. perpendicular to the beam direction even in the laboratory frame. The $J / \psi$ vertex position in this plane can therefore also be used to identify the primary decay products of the tagging $B$, as illustrated in Figure 5.1. The difference in decay position along the beam direction between the $J / \psi$ vertex and the primary vertex for the tagging $B$ then measures the relative time betwecn decays $t=t_{2}-t_{1}$ referred to in the previous section.
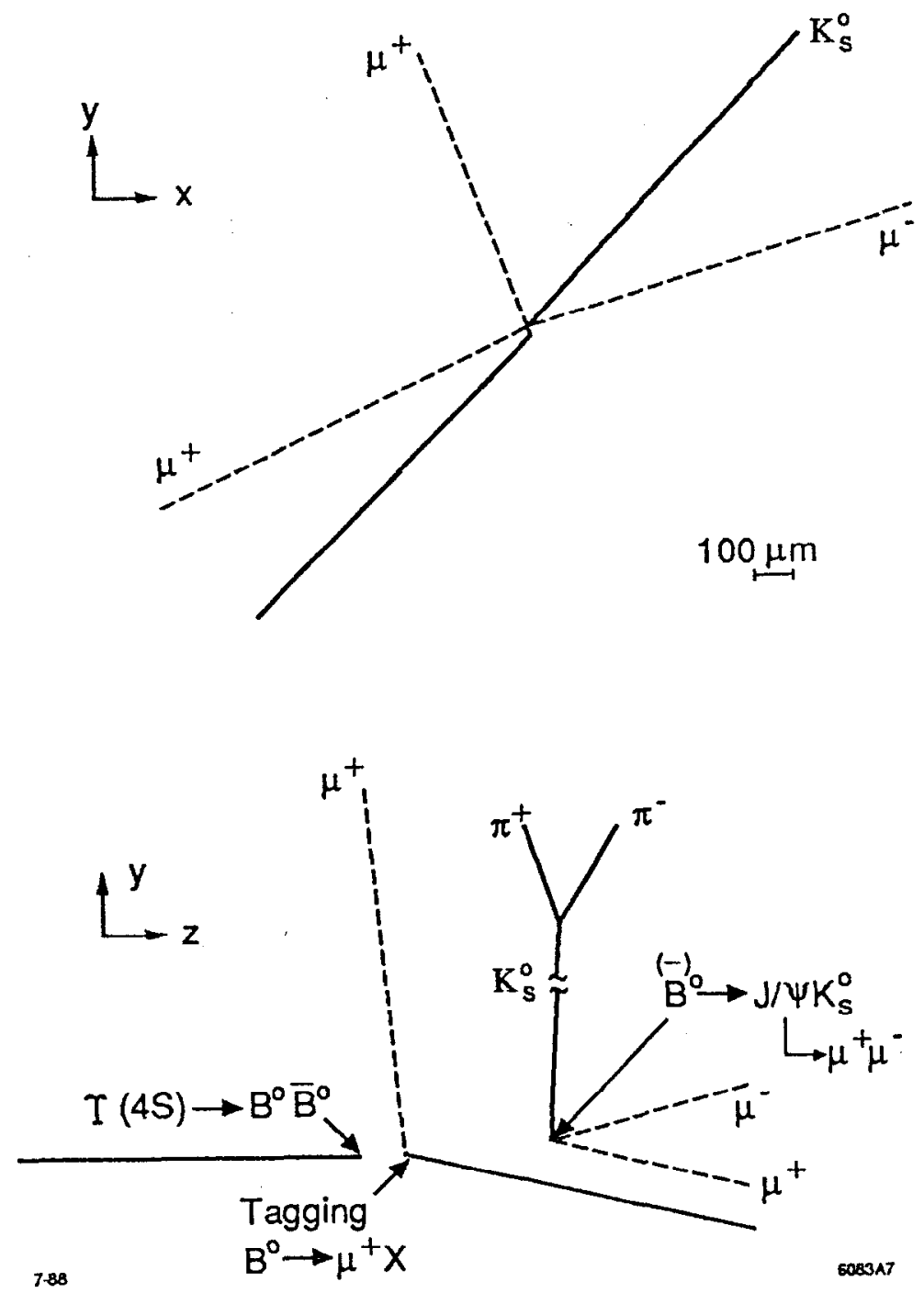

We use charged $K$ 's or charged leptons to identify the tagging $B$ as a $B^{0}$ or $\bar{B}^{0}$. Throughout this analysis we assume perfect particle identification. We will discuss the background 
levels which would result from particle misidentification.

We impose the following fiducial cuts on all charged tracks: they must have $p_{T} \geq 100 \mathrm{MeV}$, and $|\cos \theta| \leq 0.98$ (in the laboratory frame). Where appropriate, we will discuss the effects of relaxing or tightening these requirements. We have not made use of constrained fits in this analysis.

Reconstructing the $J / \psi$ and the $K_{S}^{0}$

In order to test the analysis, we generated various runs of 2000 or $20000 B^{0} \bar{B}^{0}$ events (for different boosts and beam pipe configurations) in which one of the $B$ 's decays to $J / \psi K_{S}^{0}$, and the $J / \psi$ decays leptonically. If we assume a branching fraction of $5 \times 10^{-4}$ for $B \rightarrow J / \psi K_{S}^{0}$, and a $J / \psi$ leptonic branching ratio of $14 \%$, this represents approximately $30 \mathrm{fb}^{-1}$ or $300 \mathrm{fb}^{-1}$, respectively $\left(1.4 \times 10^{7}\right.$ or $1.4 \times 10^{8} B^{0} \bar{B}^{0}$ events $)$. This assumption for the $B \rightarrow J / \psi K_{S}^{0}$ branching fraction is based on the measured branching fraction ${ }^{15}$ of $(8 \pm 3) \times 10^{-4}$ for $B^{ \pm} \rightarrow J / \psi K^{ \pm}$. Unless otherwise noted, all numbers presented in the remainder of this section are from a run of 20000 events with $\beta \gamma=0.56$, and a $10 \mathrm{~mm}$ beampipe radius.

Since the extreme cleanliness of this decay mode is due to the clear signature of the $J / \psi$ decaying to an oppositely charged pair of leptons, our first step is to reconstruct this state. We accept any $e^{+} e^{-}$or $\mu^{+} \mu^{-}$pair whose invariant mass is between 3.0 and $3.2 \mathrm{GeV}$ to be a $J / \psi$ candidate. The $J / \psi$ candidate is retained if the leptons form a single vertex, with $\chi^{2} \leq 12$ for the vertex fit. We find at least one $J / \psi$ candidate which satisfies these selection criteria in $90 \%$ of the events. In about $1 \%$ of the events, we find more than one candidate; this ambiguity is resolved later.

We have assumed that we can identify both electrons and muons over the full range of momenta from $J / \psi$ decays; i.e., $0.5 \mathrm{GeV} / \mathrm{c}$ to $4.5 \mathrm{GeV} / \mathrm{c}$. Electrons pose no problem; muons may be more of a challenge in the low momentum range. Čerenkov counters and $\mathrm{dE} / \mathrm{dx}$ can provide $\mu / \pi$ separation up to a momentum of about 500 or $600 \mathrm{McV} / \mathrm{c}$; a combi nation of muon range chambers and coarser muon counters and absorber can probably cover the rest of the range. If, for example, muons cannot be cleanly identified in the 500 to 
$800 \mathrm{MeV} / \mathrm{c}$ range, about $6 \%$ fewer $J / \psi K_{S}^{0}$ events are reconstructed.

The next step is to find a $K_{S}^{0}$ either in the $\pi^{+} \pi^{-}$or $\pi^{0} \pi^{0}$ decay mode. For the easier (and dominant) charged mode, we simply calculate the invariant mass of all pairs of oppositely charged pions. We use rather loose cuts on invariant mass and vertex $\chi^{2}$ for the charged pion mode, and on invariant mass and $\pi^{0}$ momentum for the neutral mode. Together, we find an average of $3.3 K_{S}^{0}$ candidates per event.

Reconstructing the $B$ from the $J / \psi K_{S}^{0}$

Once the $J / \psi$ ('s) and $K_{S}^{0}$ ('s) have been found, we calculate the invariant mass of each combination in the event. This invariant mass distribution is shown in Figure 5.2 for all the $J / \psi K_{S}^{0}$ candidates. We boost the $J / \psi K_{S}^{0}$ combination back to the $\Upsilon(4 \mathrm{~S})$ rest frame to determine the total momentum $p_{c m}$ of the $J / \psi K_{S}^{0}$ combination in this frame. A combination

Our net efficiency for reconstructing $B \rightarrow J / \psi K_{S}^{\circ}$ is $65 \%$. is defined to be a $B$ candidate if $p_{c m} \leq 450 \mathrm{MeV} / \mathrm{c}$, and the reconstructed mass of the combination lies between 5.15 and $5.45 \mathrm{GeV}$. (We cut more tightly on the lower edge of the mass region in order to eliminate background from $B \rightarrow J / \psi K_{S}^{0} \pi^{0}$ which has opposite sign $\mathrm{CP}$; we will discuss this in more detail below.) We do not require that the $J / \psi$ decay products come from an isolated vertex. We find at least one combination that meets these criteria in $68 \%$ of the events. If we require that all charged and neutral particles have $|\cos \theta| \leq 0.95$, this drops to $56 \%$; it drops even further, to $41 \%$, if we cut at $|\cos \theta| \leq 0.90$ (these efficiencies decrease more quickly at a higher boost; see section 5.7). This clearly demonstrates the need for good forward particle detection, down to within $\sim 12^{\circ}$ of the beamline, and mandates that the energy asymmetry not be too large. 


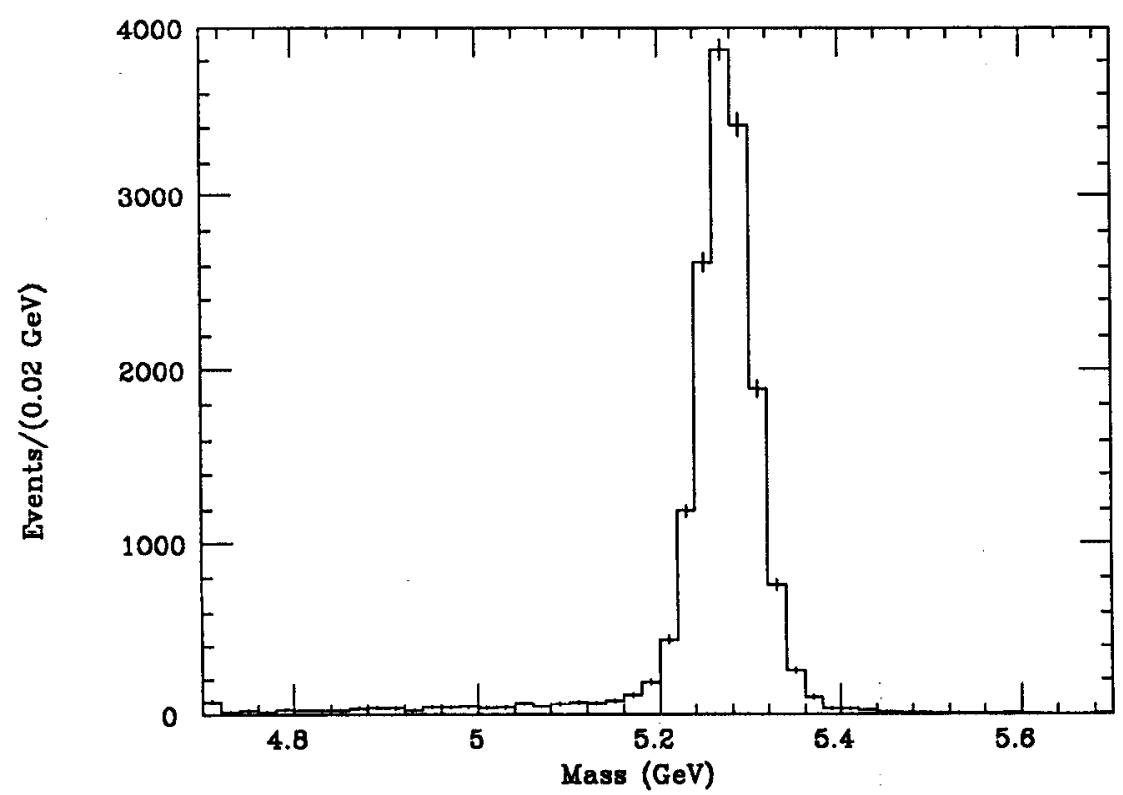

In about $3 \%$ of the events, we find more than one combination which passes the selection criteria. These ambiguities are resolved by choosing the "best" combination, as determined by mass and vertex $\chi^{2}$. Note that choosing the correct $K_{S}^{0}$ is actually unimportant, as long as our cuts are not so loose that they allow background events from other processes. The correct choice of $J / \psi$ candidate is, however, very important since the $J / \psi$ candidate determines the vertex positions of the $B$ mesons.

Finding the Primary Vertex of the Tagging $B$

Once a $B \rightarrow J / \psi K_{S}^{0}$ candidate has been identified, we proceed to find the primary vertex of the tagging $B$. All charged tracks in the event, except the leptons from the $J / \psi$ candidate and the pions from the $K_{S}^{0}$ candidate, are considered. One "track", calculated from the vertex parameters of the $J / \psi$ candidate leptons, is included. We then group these tracks into vertices. As explained above, the primary vertices of the two $B$ 's will be very close to each other in the plane transverse to the beam direction. The separation of the two vertices in the transverse plane is determined by the transverse momenta and the lifetimes of the $B$ 's. We use the measured transverse momentum of the $J / \psi K_{S}^{0}$ candidate and the mean $B$ lifetime to translate the measured $J / \psi$ vertex to the mean position of the primary vertex for the tagging $B$ in the
Figure 5.2. Invariant mass distribution for $J / \psi K_{S}^{0}$ candidates in events in which one $B$ decays to $J / \psi K_{S}^{0}$.
We use the $J / \psi$ vertex of the completely reconstructed $B$ to help determine the position of the primary vertex of the other $B$. 
transverse plane; the translated $J / \psi$ vertex, of course, tells us nothing about the $z$ position of the tagging $B$ decay. Those tracks that form a good vertex with the translated $J / \psi$ vertex determine the primary vertex of the tagging $B$, and hence its decay position.

The Tagging $B$

We now need to identify the tagging $B$ as a $B^{0}$ or a $\bar{B}^{0}$. Two techniques have been used: lepton tagging, which uses semileptonic decays of the $B$, and charged kaon tagging, which uses charged $K$ 's from the bottom $\rightarrow$ charm $\rightarrow$ strange cascade. A negative lepton or kaon identifies the tagging $B$ as a $\bar{B}^{0}$, while a positive lepton or kaon indicates a $B^{0}$ (where we have used the convention that a $B^{0}$ has quark content $\bar{b} d$ ).

\section{Lepton Tagging}

To correctly identify the tagging $B$ as a $B^{0}$ or $\bar{B}^{0}$, we must select leptons from the primary decay of the $B$ meson. These leptons tend to have higher momentum in the $B$ rest frame than leptons from secondary decays such as the decay of the charm quark. We boost the momentum of each lepton back into the $\Upsilon(4 S)$ rest frame. We accept any lepton with momentum in the $\Upsilon(4 \mathrm{~S})$ rest frame greater than $1.4 \mathrm{GeV}$. There is very little contamination from wrong-sign leptons in this sample. In addition, we accept leptons with momentum between 0.8 and $1.4 \mathrm{GeV}$ if exactly two vertices are found for the tagging $B$ and the lepton belongs to the primary vertex (i.e., the same vertex as the translated $J / \psi$ vertex). Of the leptons with momentum between 0.8 and $1.4 \mathrm{GeV}$, about $27 \%$ satisfy this vertex requirement. Events with only one lepton satisifying the above criteria are accepted as tagged events. The total tagging efficiency is $14 \% ; 94 \%$ of the tags are correct.

Kaon tagging

Besides the cascade decay which can lead to a charged kaon useful for tagging, we must consider other sources of charged $K$ 's in $B$ decays. The $b \rightarrow c$ decay $B \rightarrow D_{s} \bar{D} X$ - can result in a wrong-sign kaon; it is likely to be accompanied by one or more other charged or neutral $K$ 's, however. In events with a single charmed meson, the Cabibbo-suppressed decays 
will generally result in two kaons (either oppositely charged, one wrong-sign charged and one neutral, or both neutral), or no kaons at all. Doubly-Cabibbo-suppressed decays of the charm meson (which fortunately are relatively rare) can give a single wrong-sign $K . b \rightarrow u$ decays of the $B$ can add a kaon of the right sign.

Hence we accept two cases. If there are two charged kaons of the same sign, and a total of no more than three charged $K$ 's, we accept that sign as tagging the $B$. If there is a single charged $K$, and no good $K_{S}^{0} \rightarrow \pi^{+} \pi^{-}$candidates, other than the one from the $B \rightarrow J / \psi K_{S}^{0}$ candidate, we again accept the charged $K$ as a good tag. This method tags $11 \%$ of the events containing a $B \rightarrow J / \psi K_{S}^{0}$ candidate $(37 \%$ with a single $K$, $4 \%$ with two or three $K$ 's), with $94 \%$ of the tags being correct.

\section{Combined tagging}

There is some overlap of the two tagging methods. In the $6 \%$ of events where both tags are available, they agreed on the identity of the $B$ meson as a $B^{0}$ or $\bar{B}^{0} 94 \%$ of the time (the 62 events in which they disagreed were considered untagged). Overall, we tag $51 \%$ of the events containing a $B \rightarrow J / \psi K_{S}^{0}$ candidate with $94 \%$ of the tags being correct. The combined reconstruction and tagging efficency is $34.6 \pm$ $0.4 \%$. The efficiency for tagging is less sensitive to the track $|\cos \theta|$ cut than is the $B \rightarrow J / \psi K_{S}^{0}$ reconstruction efficiency. It drops from $51 \%$ for $|\cos \theta| \leq 0.98$ to $49 \%$ for $|\cos \theta| \leq 0.95$, and to $46 \%$ for $|\cos \theta| \leq 0.90$.

Backgrounds to $B \rightarrow J / \psi K_{S}^{0}$ Reconstruction

Although the $B \rightarrow J / \psi K_{S}^{0}$ signature is very distinctive, the branching fraction is relatively small $\left(\approx 5 \times 10^{-4}\right)$. Therefore, this mode could have significant backgrounds. We have investigated three possible sources of background: events in which a hadron is misidentified as a lepton to form a $J / \psi$ candidate, events in which both $B$ 's decay semileptonically, and events in which $B \rightarrow J / \psi K_{S}^{0} \pi^{0}$, a process which can also be used for measuring $\mathrm{CP}$ violation but which has opposite sign $\mathrm{CP}$ and therefore must be well-separated from $B \rightarrow J / \psi K_{S}^{0}$.

\section{Particle Misidentification}

We generated a variety of events, including events in which
We tag $51 \%$ of the events containing a $B \rightarrow J / \psi K_{S}^{0}$ candidate, with $94 \%$ of the tags being correct. 
Particle misidentification contributes negligible background to the reconstruction of the $B \rightarrow J / \psi K_{S}^{0}$ candidate.

The background from events with two semileptonic $B$ decays is less than $1.5 \%$.

The decay $B \rightarrow J / \psi K_{S}^{0} \pi^{0}$, which has CP opposite to $J / \psi K_{S}^{0}$, contributes a background of less than $1.5 \%$. one or both $B$ mesons decay semileptonically, and associated the leptons with pions in the event to attempt to reconstruct a $J / \psi$ candidate. For a particle misidentification probability of $1 \%$, we find no candidates for $B \rightarrow J / \psi K_{S}^{0}$, resulting in an estimate of less than $2.0 \%$ background at the $90 \%$ confidence level.

Events with two semileptonic decays

Since the branching ratios for $B \rightarrow e^{ \pm} X$ and $B \rightarrow \mu^{ \pm} X$ are each roughly $12 \%$, we expect the rate for same-flavor double semileptonic events to be almost $3 \%$, about 400 times larger than the $B \rightarrow J / \psi K_{S}^{0}, J / \psi \rightarrow l^{+} l^{-}$rate per event. In order to measure the background from this process, events with two semileptonic $B$ decays were generated at random. A preselection was then done to choose only those events in which same-flavor opposite sign leptons had an invariant mass within $200 \mathrm{MeV}$ of the $J / \psi$ mass, and in which there was at least one $K_{S}^{0}$. In order to get 2000 such events, over 121,000 double semileptonic events were generated. Thus the 2000 events represent 2.2 million $B^{0} \bar{B}^{0}$ events. None of these events pass our selection criteria for a $B \rightarrow J / \psi K_{S}^{0}$ candidate. Normalizing to the number of events in the signal channel, we estimate a background from double semileptonic decays of less than $1.5 \%$ at the $90 \%$ confidence level. We estimate that events in which one $B$ decays semileptonically, and then the charm state also decays semileptonically, produce fake $J / \psi$ 's at only $10 \%$ to $20 \%$ of the rate for events in which both $B$ 's decay semileptonically.

$\underline{B \rightarrow J / \psi K_{S}^{0} \pi^{0}}$

We expect the product branching ratio ${ }^{15}$ for $B \rightarrow J / \psi K^{* 0}$, $K^{* 0} \rightarrow K_{S}^{0} \pi^{0}$ to be about equal to the $B \rightarrow J / \psi K_{S}^{0}$ rate. Thus we simulated 2000 events in which one $B$ decays to $J / \psi K^{* 0}$ and every $K^{* 0}$ decays to $K_{S}^{0} \pi^{0}$. Only 18 events pass our selection criteria for $J / \psi K_{S}^{0}$ candidates. The background rate is primarily a function of the detector's mass resolution, which in turn is a function of the momentum resolution of the tracking chambers and the energy resolution of the calorimeter. These 18 events represent a background of less than $1.5 \%$ which is not significant. 
Backgrounds to Tags

Therc are two possible reasons for incorrectly tagging the $B$ as a $B^{0}$ or $\bar{B}^{0}$. First, the charged lepton or kaon may be correctly identified but nevertheless have the wrong sign because of secondary or Cabibbo-suppressed decays. As mentioned above, this background is about $6 \%$ for the lepton and kaon tags. Secondly, a wrong-sign particle may be misidentified as a lepton or kaon. The particle misidentification probability must be low enough such that the background from the second source is significantly less than the background from the first source.

Misidentifying a charged kaon as a lepton does not introduce a background to the lepton tag because most charged kaons which pass the momentum cuts used for lepton tagging have the same sign as the lepton from primary $B$ decay. It was determined that the number of wrong-sign charged pions and the number of right-sign leptons which meet the selection criteria for a lepton tag are about the same in events with a reconstructed $B \rightarrow J / \psi K_{S}^{0}$ candidate. Therefore, if the probability of misidentifying a pion as a lepton is less than about $2 \%$, this background to the lepton tag will be less than half of that due to wrong-sign leptons.

Since $6 \%$ of the charged kaon tags are actually of the wrong sign, and we find a $K^{ \pm} \operatorname{tag}$ in $41 \%$ of the reconstructed events, this combination produces $2.5 \%$ wrongly tagged events. In addition, if we ignore charged pions from $K_{S}^{0}$ decays, there are an average of 1.7 wrong-sign pions per event. This means for every $1 \%$ probability of misidentifying a pion as a kaon, we would have an additional $1.7 \%$ wrongly tagged events. This indicates that the $K / \pi$ misidentification rate must be kept to $\sim 1 \%$ for momenta up to $3 \mathrm{GeV} / \mathrm{c}$ to prevent the misidentified pions from dominating our rate of false tags. A Čerenkov ring-imaging device will probably be required if $K$ tagging is to be used.

It should be noted that if one is not able to obtain such a low misidentification rate with high efficiency for kaon identification, one could instead use $D$ tagging. We estimate that by using $D$ mesons (reconstructed in their simpler decay modes, such as $K^{+} \pi^{-}\left(\pi^{0}\right), K^{+} \pi^{-} \pi^{-}\left(\pi^{0}\right), K^{+} \pi^{-} \pi^{+} \pi^{-}$,
The $K / \pi$ misidentification rate must be kept to $\sim 1 \%$ for momenta up to $3 \mathrm{GeV} / \mathrm{c}$.

If $K$ tagging is not practical, a combination of $D$ tagging and lepton tagging could provide a tagging efficiency of $30 \%$. 
The size of the background due to tags can be estimated from the data itself, using pairs of charged $B$ mesons.
$K_{S}^{0} \pi^{-}$, etc.), together with the lepton tags, $30 \%$ of the events can be tagged with only $5 \%$ wrong tags.

Correction to Measured Asymmetry due to Backgrounds

Although the backgrounds dilute the measured asymmetry, the effect is fully correctable if we can accurately estimate the size of each background. For wrong sign tags, which move events from one time distribution to the complementary distribution, the background has a doubly strong dilution. The measured asymmetry $A_{\text {meas }}$ is related to the true asymmetry $A_{\text {true }}$ by the relation

$$
A_{\text {meas }}=A_{\text {true }}(1-2 P)
$$

where $P$ is a measure of the fraction of the sample which is background (for other types of random backgound, the factor of 2 is absent from the formula). For example, for backgrounds due to wrong-sign lepton or kaon tags, $P$ is the probability that a tag has the wrong sign. As we have shown, this probability is about $6 \%$. For backgrounds due to misidentified lepton or kaon tags, $P$ is the particle misidentification probability per event times the ratio of untagged to tagged events. For the analysis outlined above, the ratio of untagged to tagged events is one.

The size of backgrounds to tags can be estimated from the data itself using pairs of charged $B$ mesons. Since the charged $B$ mesons cannot mix, the only sources of two like-sign tags in an event are secondary decays and particle misidentification. We would like to know the correction to $A_{\text {meas }}$ due to backgrounds to significantly better than $10 \%$. Since the corrections themselves are generally not larger than $10 \%$, this should not pose a problem.

In order to minimize the statistical error in the asymmetry measurement, the tagging strategy must be optimized. This requires balancing high tagging efficiency against a higher percentage of incorrect tags. This optimization would depend on the size of the data sample and the precision required. While fairly "loose" tagging would be very useful in an initial search with a small data sample, cleaner, less efficient methods might be preferable for larger data sets and higher precision. 


\section{Summary of Events Generated and Reconstructed}

In Table 5.1, we summarize the number of events generated for this analysis, the assumed branching fractions and the size of the equivalent $B^{0} \bar{B}^{0}$ data sample, the charged and neutral track geometrical criteria, the number of $B \rightarrow J / \psi K_{S}^{0}$ candidates and, finally, the number of tagged events. For $B$ decay to the CP eigenstate $J / \psi K_{S}^{0}$, the size of the final correctly-tagged sample is 6527 events for $1.4 \times 10^{8}$ produced $B^{0} \bar{B}^{0}$ pairs, which would be obtained with an integrated luminosity of $\sim 200 \mathrm{fb}^{-1}$.

Table 5.1 Summary of results for the CP eigenstate $J / \psi K_{S}^{0}$, with $\beta \gamma=0.56$

Number of generated events with one

$B \rightarrow J / \psi K_{S}^{0}$ decay 20000

Assumed branching fractions for:

$$
\begin{array}{lc}
B \rightarrow J / \psi K_{S}^{0} & 5 \times 10^{-4} \\
J / \psi \rightarrow l^{+} l^{-} & 0.14
\end{array}
$$

Equivalent number of produced $B^{0} \bar{B}^{0}$ pairs

$1.4 \times 10^{8}$

Charged track criteria:

minimum transverse momentum

$0.1 \mathrm{GeV} / \mathrm{c}$

maximum $|\cos \theta|$

0.98

Neutral track criteria:

minimum energy

$0.03 \mathrm{GeV}$

maximum $|\cos \theta|$

0.99

Number of $B \rightarrow J / \psi K_{S}^{0}$ candidates 13620

Number of right-sign lepton tags

Number of wrong-sign lepton tags

Number of right-sign kaon tags 5286

Number of wrong-sign kaon tags

Total number of right-sign tags 
Fitting for the amplitude of the CP-violating asymmetry reduces the error by $15-20 \%$.

\section{Measuring CP Violation}

To measure the CP-violating asymmetry, we use the timeordering of the decays determined by the relative positions of the $J / \psi$ vertex and the tagging $B$ vertex along the beam direction and the identity of the tagging $B$ from the $K^{ \pm}$or lepton tag to divide the events into the four categories described in section 5.2 ( $B^{0}$ first, $J / \psi K_{S}^{0}$ second; $J / \psi K_{S}^{0}$ first, $B^{0}$ second; $\bar{B}^{0}$ first, $J / \psi K_{S}^{0}$ second; $J / \psi K_{S}^{0}$ first, $\bar{B}^{0}$ second). Combining the first and fourth categories, and the second and third categories, we plot the $\Delta z$ distribution (the difference in position of the $J / \psi$ vertcx and the tagging $B$ vertex along the beam direction), which is proportional to the relative decaytime, to a good approximation.

Given 6914 reconstructed and tagged events, we find the raw asymmetry in the number of events to be $-0.179 \pm 0.012$. If $\Delta m / \Gamma=0.75$, this translates into a measurement of $\sin 2 \beta$ of $-0.424 \pm 0.028$ (after correcting for $6 \%$ wrong tags) using this integrated asymmetry only. We can do better by performing a simultaneous fit to the two time distributions to derive $\sin 2 \beta$ itself; this reduces the error to \pm 0.023 ; the fit is shown in Figure 5.3. Known values of $\Delta m / \Gamma$ and the $B^{0}$ lifetime must be provided to the fit; these will be well measured by the time this measurement is attempted. The fitting function also takes into account the imperfect vertex position resolution; a measured value for the standard deviation of the $\Delta z$ measurement must also be provided. For our sample of 20000 events in which one $B$ meson decays to $J / \psi K_{S}^{0}$, we measure $\sin 2 \beta$ to be $-0.408 \pm 0.023$, in excellent agreement with the input value of -0.4 . 

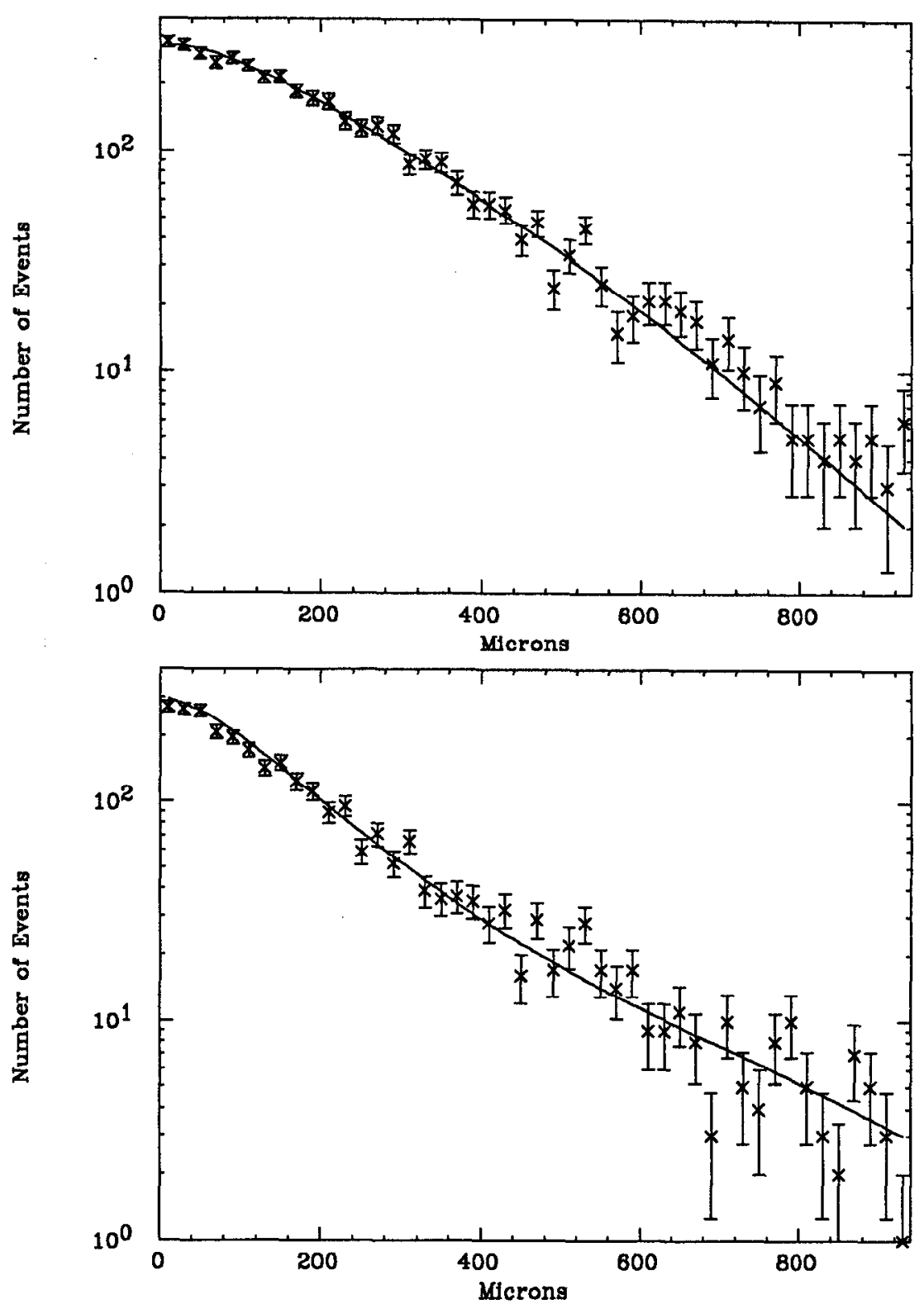

Of course, if $\sin 2 \beta$ is this large, a much smaller data sample would be needed to reach our goal of a three standard deviation measurement. In another sample of 2000 events (corresponding to $30 \mathrm{fb}^{-1}$ ), the analysis yields 695 reconstructed and tagged events. The integrated method yields $\sin 2 \beta=-0.25 \pm 0.09$, while the fitting method yields $\sin 2 \beta=$ $-0.37 \pm 0.07$, which is a five standard deviation measurement. Again, this shows a $\sim 20 \%$ reduction in the error compared to the integrated measurement.
Figure 5.3 The decay length distributions for the two classes of events; the upper plot includes events where the first $B$ decays to $J / \psi K_{S}^{0}$ and the second is tagged as a $B^{0}$, or the first is tagged as a $\bar{B}^{0}$ and the second is $B \rightarrow J / \psi K_{S}^{0}$ (types (1) and (4)); the lower plot includes events where the first $B$ is tagged as a $B^{0}$ and the second is $B \rightarrow J / \psi K_{S}^{0}$, or the first $B$ decays as $J / \psi K_{S}^{0}$ and the second is tagged as a $\bar{B}^{0}$; (types (2) and (3)). The events were generated with $\sin 2 \beta=$ -0.40 and $\beta \gamma=0.56$; the fit found $\sin 2 \beta=-0.408 \pm 0.023$. 
This measurement requires good momentum resolution, particle identification and vertex detection, although it should be noted that other analyses actually make more stringent demands on the vertex detector
The integrated luminosity needed to establish a $3 \sigma$ effect should be between 4 and $160 \mathrm{fb}^{-1}$, but only for an asymmetric machine

\section{Detector Requirements}

In order to achieve this type of measurement a very good detector, such as has been described in Chapter 3 , will be needed. We reemphasize here those characteristics of the detector which most important to this analysis. Vertex detection is central: a detector with excellent resolution and as small a beampipe radius as possible is desirable. Note, however, that in this analysis it is important only to get the time order of the decays correct. For events in which $|t-\bar{t}|$ is small, the asymmetry is near zero, so the integrated asymmetry is fairly insensitive to the resolution in the decay length. ${ }^{16}$ Other analyses make more stringent requirements on the vertex measurements. Very good momentum resolution is needed for good invariant mass discrimination (e.g., separating $B \rightarrow$ $J / \psi K_{S}^{0}$ from $\left.B \rightarrow J / \psi K_{S}^{0} \pi^{0}\right)$. Electron, muon and kaon identification at momenta from a few hundred $\mathrm{MeV} / \mathrm{c}$ to a few $\mathrm{GeV} / \mathrm{c}$ are all important for reconstructing the $J / \psi$ and for lepton and kaon tagging. Finally, good photon detection and energy resolution are useful for the reconstruction of $\pi^{0}$ 's, which can be used for finding more $K_{S}^{0}$ 's, or for reconstructing other CP eigenstates (e.g., $\left.J / \psi K_{S}^{0} \pi^{0}\right)$.

Required Data Sample

In order to meet our goal of a three standard deviation measurement of $\sin 2 \beta$, we need roughly $N=33 /(\sin 2 \beta)^{2}$ reconstructed and tagged events. As we have shown, our analysis yields about 23 such events per $\mathrm{fb}^{-1}$, if we use only the CP eigenstate $J / \psi K_{S}^{0}$. The total luminosity needed then is $L d t=1.6 \mathrm{fb}^{-1} /(\sin 2 \beta)^{2}$, or between $\sim 4$ and $\sim 160 \mathrm{fb}^{-1}$. Aleksan et $a l^{5}$. have shown that the states $J / \psi K^{* 0}$ and $\psi^{\prime} K_{S}^{0}$ can be reconstructed with similar efficiency. Considering product branching ratios, these $\mathrm{CP}$ eigenstates could easily double the number of reconstructed and tagged events available to measure $\sin 2 \beta$. Other CP eigenstates (such as $\left.D^{+} D^{-}\right)^{17}$ which can can be measured with a significance comparable to $J / \psi K_{S}^{0}$, can be used either to provide consistency chccks, to increase the statistical significance of the CP-violation measurement, or, perhaps most importantly, to allow exploration of the pattern of CP violation. It is the comparison of a number of measurements of CP-violating quantities 
(see Chapter 2) which can provide a sensitive consistency test of the Standard Model, or provide clues to the origin of effects which go beyond it.

\subsection{Measuring $\sin 2 \alpha$ With $B \rightarrow \pi^{+} \pi^{-}$}

The decay $B \rightarrow \pi^{+} \pi^{-}$can be used to measure $\sin 2 \alpha$ where $\alpha$ is one of the angles in the unitarity triangle discussed in Chapter 2. Since the $\pi^{+} \pi^{-}$vertex marks the decay of the $B$ just as the lepton pair from $J / \psi$ decay marks the $B$ decay in $B \rightarrow J / \psi K_{S}^{0}$, the results of the $B \rightarrow J / \psi K_{S}^{0}$ study can be used to estimate that approximately 1000 reconstructed, tagged $B \rightarrow \pi^{+} \pi^{-}$events are needed to measure $\sin 2 \alpha$ to an absolute precision of 0.06 . Since the uncertainties on $\sin 2 \alpha$ are so large, we believe this is a reasonable goal.

In a study discussed in Section 6.5, the reconstruction efficiency and backgrounds for the mode $B \rightarrow \pi^{+} \pi^{-}$are estimated. The reconstruction efficiency is $\sim 35 \%$ and that the fraction of continuum background events which pass the sclection criteria is $\sim 5 \times 10^{-7}$. The most serious background is from $B \rightarrow \pi^{ \pm} K^{\mp}$ and $B \rightarrow K^{+} K^{-}$. Because these decays are all two-body, the $\pi^{ \pm}$and $K^{ \pm}$momenta are high (in the range $2-4 \mathrm{GeV}$ ) and therefore particle identification is difficult without a Cerenkov ring imaging detector (CRID). With a CRID, the backgrounds should be acceptable.

Assuming a reconstruction efficiency of $35 \%$, a tagging efficiency of $50 \%$ (the tagging efficiency determined in the $B \rightarrow J / \psi K_{S}^{0}$ study), and a sample of $30 \mathrm{fb}^{-1}$ at the $\Upsilon(4 \mathrm{~S})$ (about $1.5 \times 10^{7} B^{0} \bar{B}^{0}$ pairs), the branching fraction for $B \rightarrow$ $\pi^{+} \pi^{-}$must be at least $2 \times 10^{-4}$ to result in a reconstructed, tagged sample of 1000 events. Theoretical models do not indicate that this is likely. To achieve a sample of 1000 events with $300 \mathrm{fb}^{-1}$ would require a branching fraction of only $2 \times 10^{-5}$ which seems more likely. ${ }^{18}$

Therefore, a measurement of $\sin 2 \alpha$ to a precision of 0.06 would require a data sample of $300 \mathrm{fb}^{-1}$ unless the branching fraction for $B \rightarrow \pi^{+} \pi^{-}$is significantly greater than $2 \times 10^{-5}$.
The position of the $B$ decay to a $C P$ eigenstate is measured with the $\pi^{+} \pi^{-}$vertex; the remaining tracks in the event identify the position of the tagging $B$ decay.

With a sample of $300 \mathrm{fb}^{-1}$, a good measurement of $\sin 2 \alpha$ seems possible. 
$A$ measurement of $\sin 2 \gamma$ can only be done in the $B_{\text {s }}$ system, and therefore must be done above the $\Upsilon(4 S)$.

\subsection{Measuring $\sin 2 \gamma$ With $B_{s} \rightarrow \rho^{0} K_{S}^{0}$}

In order to determine the third angle, $\gamma$, of the unitarity triangle (as discussed in Chapter 2), it is necessary to produce $B_{s}$ mesons, and therefore to run above $B_{s} \bar{B}_{s}$ threshhold. In particular, this means running at an energy higher than the $\Upsilon(4 \mathrm{~S})$ resonance. In this section we will assume that this running is done at the $\Upsilon(5 \mathrm{~S})$, and that the $\Upsilon(5 \mathrm{~S})$ is also above $B_{s}^{*} \bar{B}_{s}^{*}$ threshhold.

The time-dependent CP-violating asymmetry in the decay $B_{s} \rightarrow \rho^{0} K_{S}^{0}$ is given by

$$
A(t)=\sin 2 \gamma \sin \left(x_{s} t / \tau\right)
$$

where $t$ is the time difference between decays to the CP eigenstate $\rho^{0} K_{S}^{0}$ and to a tagged $B_{s}$ decay, and $x_{s}$ is the mixing parameter for the $B_{s}$ system $\left(x_{s}=\Delta m / \Gamma\right)$. Since $x_{s}$ is expected to be large $(Z 3)$, the asymmetry will oscillate in time very rapidly. ${ }^{\# 1}$ Imperfect vertex resolution will, therefore, cause the oscillations to be smeared and the asymmetry to be diluted. The resolution must be well understood to predict the amount by which the asymmetry is diluted. These issues will be addressed below.

The branching fraction $B_{s} \rightarrow \rho^{0} K_{S}^{0}$ times the efficiency for reconstructing this final state must be large enough to produce a sufficient number of events. The minimum required number of events will be discussed in the first section, while reconstruction efficiency and backgrounds will be discussed in the second section.

Just as in the measurement of the mixing parameter $x_{s}$, the CP-violation measurement suffers from the complication of the $B_{s} \bar{B}_{s}$ pair being produced in both a $\mathrm{C}=-1$ and $\mathrm{C}=+1$ state. This issue will be discussed in the third section.

\#1 We assume that $x_{s}$, and therefore the frequency of the oscillation, will be measured using like-sign leptons as discussed in section 6.2 on $x_{s}$. Therefore, we will be trying to determine the amplitude of the oscillation only. 
Requirements for Sample Size and Resolution

Using the same notation as in the discussion of measuring $C P$ violation in the decay $B \rightarrow J / \psi K_{S}^{0}$, the rate for $f_{C P}\left(t_{1}\right) f_{B}\left(t_{2}\right)$ or $f_{\bar{B}}\left(t_{1}\right) f_{C P}\left(t_{2}\right)$ is proportional to

$$
e^{-t / \tau}\left[1+\sin 2 \gamma \sin \left(x_{s} t / \tau\right)\right]
$$

while the rate for $f_{B}\left(t_{1}\right) f_{C P}\left(t_{2}\right)$ or $f_{C P}\left(t_{1}\right) f_{\bar{B}}\left(t_{2}\right)$ is proportional to

$$
e^{-t / \tau}\left[1-\sin 2 \gamma \sin \left(x_{s} t / \tau\right)\right]
$$

The time-dependent asymmetry ${ }^{\# 2}$ is then simply

$$
A(t)=\sin 2 \gamma \sin \left(x_{s} t / \tau\right)
$$

a sinusoidal function with wavelength $2 \pi \tau / x_{s}$ and amplitude $\sin 2 \gamma$. In Figures 5.4 and 5.5, we show examples of this timedependent asymmetry for particular cases which are discussed in detail below.

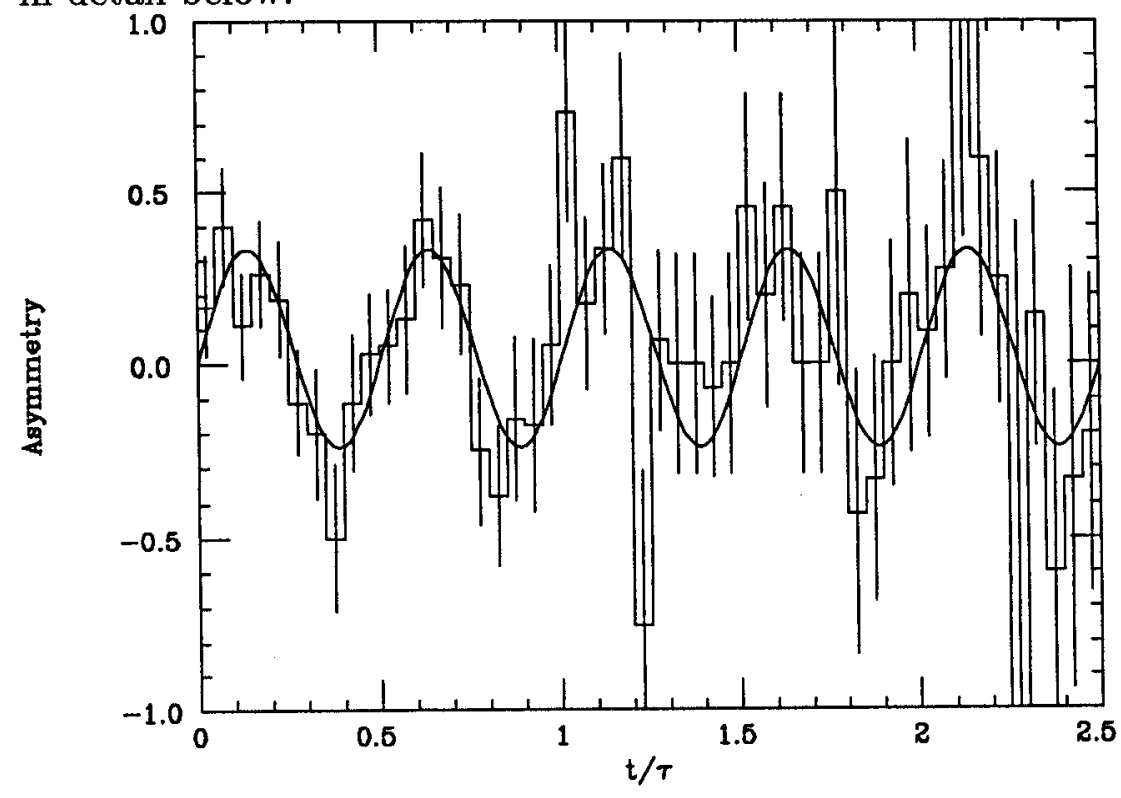

\#2 Note that when integrated over $t$, this time-ordered asymmetry is diluted by a factor of $x_{s} /\left(1+x_{s}^{2}\right)$, which is approximately $1 / x_{s}$ for large $x_{s}$ and therefore a large dilution.
Figure 5.4 Time-dependent $C P$-violating asymmetry, with $\sin 2 \gamma=0.3$ and $x_{3}=4 \pi$, using generated decay lengths for 1000 events, with perfect resolution for measuringt. The fit finds an amplitude of $0.29 \pm$ 0.04 . 
Figure 5.5 Time-dependent CP-violating asymmetry, with $\sin 2 \gamma=0.3$ and $x_{s}=4 \pi$, using generated decay lengths for 1000 events, with Gaussian smearing having $\sigma=0.2 \lambda$, where $\lambda$ is the wavelength of the oscillation. The fit finds an amplitude of $0.14 \pm$ 0.04 , showing that even that with this modest smearing, the amplitude is reduced by a factor of two

The measurement of $C P$ violation in the $B_{s}$ system places more stringent requirements on vertex resolution because of the expected rapid mixing rate.

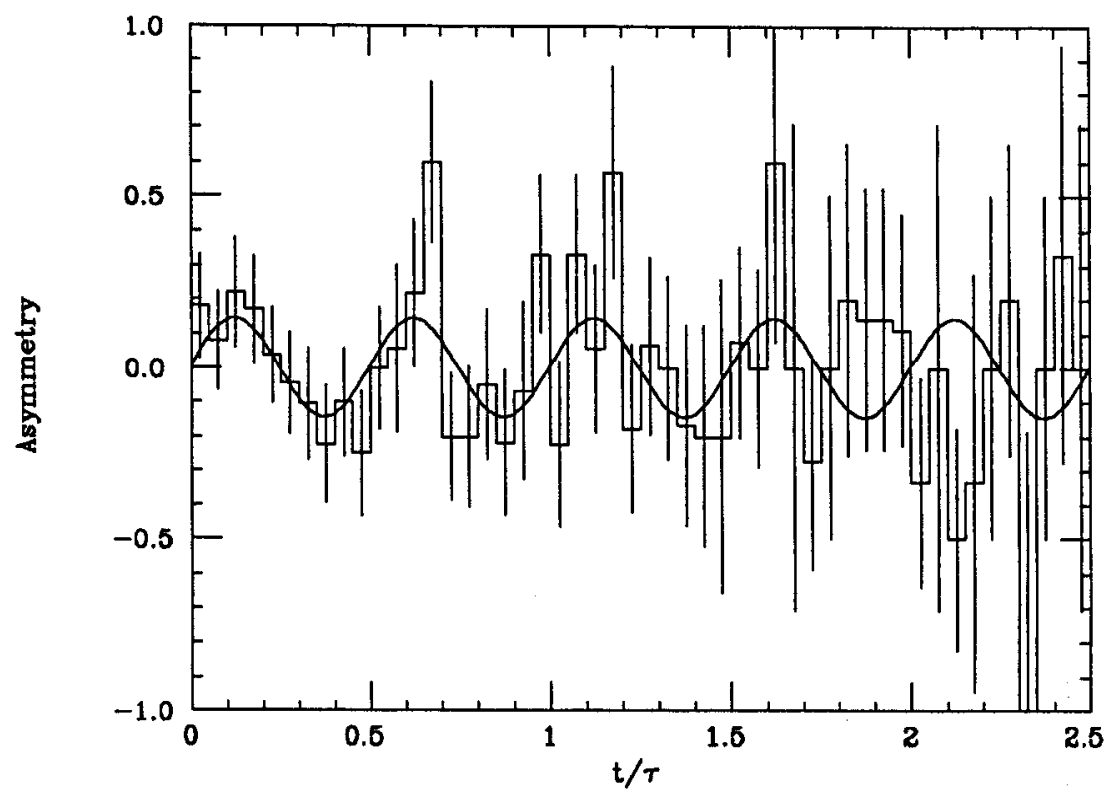

In units of distance between decay vertices, the wavelength of the oscillation $\lambda$ is given by

$$
\lambda=\frac{2 \pi \beta \gamma c \tau}{x_{s}}
$$

in the approximation that the two $B_{s}$ 's are produced at rest in the center-of-mass frame. Recall that $\beta \gamma c \tau$ is approximately $330 \mu \mathrm{m}$ for $\beta \gamma=1$. Therefore, for $x_{s}$ in the range $2 \pi$ to $4 \pi$, for example, the wavelength of the oscillation is only 165 $330 \mu \mathrm{m}$. The resolution for measuring the distance between decay vertices $\Delta z$ must be significantly better than this to prevent a significant reduction in the amplitude of the oscillation due to smearing.

To estimate the required resolution, we generated the decay lengths for 100,000 events with the expected time dependence for $\sin 2 \gamma=1$ and $x_{s}=4 \pi$. The time-dependent asymmetry was fit to a sine wave for two cases: for perfect resolution and for a spatial resolution $\sigma$ for measuring $\Delta z$ given by

$$
\frac{\sigma}{\beta \gamma c \tau}=0.1 \quad \text { or } \quad \frac{\sigma}{\lambda}=\frac{\sigma}{\beta \gamma c \tau} \frac{x_{s}}{2 \pi}=0.2 .
$$

Therefore, this would correspond to a spatial resolution of $33 \mu \mathrm{m}$ for $\beta \gamma=1$, or $18 \mu \mathrm{m}$ for $\beta \gamma=0.56$. This resolution 
dilutes the amplitude of the oscillation by about a factor of two. If $x_{s}=2 \pi$ rather than $4 \pi$, the same dilution would correspond to a resolution of $66 \mu \mathrm{m}$ rather than $33 \mu \mathrm{m}$ (for $\beta \gamma=1$ ). In other words, to limit the dilution to less than a factor of two, we must have sufficient vertex resolution to satisfy

$$
\frac{\sigma}{\beta \gamma c \tau} \frac{x_{s}}{2 \pi} \lesssim 0.2
$$

Next we address the question of the number of reconstructed events needed to measure the asymmetry. The statistical error on an asymmetry $\mathcal{A}$ is given by

$$
\delta \mathcal{A}=\sqrt{\frac{1-\mathcal{A}^{2}}{N}}
$$

where $N$ is the total number of reconstructed events and $\mathcal{A}=$ $N_{\text {diff }} / N . N_{\text {diff }}$ is the number of events which contribute to the asymmetry. In this case, $N_{\text {diff }}$ is given by

$$
\begin{aligned}
N_{\text {diff }} & =\frac{N}{\tau} \sin 2 \gamma \int_{0}^{\infty} e^{-t / \tau}\left|\sin \left(x_{s} t / \tau\right)\right| d t \\
& =N \sin 2 \gamma \frac{x_{s}}{1+x_{s}^{2}}\left(\frac{2}{1-e^{-\pi / x_{s}}}-1\right) .
\end{aligned}
$$

For large $x_{s}(\geq 3)$,

$$
\frac{x_{s}}{1+x_{s}^{2}}\left(\frac{2}{1-e^{-\pi / x_{s}}}-1\right) \approx \frac{2}{\pi} .
$$

Therefore, $\mathcal{A} \approx(2 / \pi) \sin 2 \gamma$ and the error on $\sin 2 \gamma$ is

$$
\begin{aligned}
\delta \sin 2 \gamma & =\sqrt{\frac{\pi^{2} / 4-\sin ^{2} 2 \gamma}{N}} \\
& \approx \frac{\pi}{2 \sqrt{N}} \quad \text { for small } \sin 2 \gamma
\end{aligned}
$$

For example, if $\sin 2 \gamma$ is less than $\approx 0.3, x_{s}$ is greater than $\approx 3$, and the total number of reconstructed, tagged events is 1000 , then $\delta \sin 2 \gamma \approx 0.05$. Such an example is shown in
For large $x$, and small $\sin 2 \gamma$, the statistical error on $\sin 2 \gamma$ is $\pi /(2 \sqrt{N})$ for $N$ reconstructed, tagged events. 
We estimate the tagging efficiency for $B_{3}$ mesons to be $20 \%$ and the reconstruction efficiency for $B_{s} \rightarrow \rho^{0} K_{S}^{0}$ to be $32 \%$.
Figure 5.4, where $\sin 2 \gamma=0.3$ and $x_{s}=4 \pi$. In these histograms, we plot the bin-by-bin asymmetry and the best fit to a sinusoidal function whose wavelength is specified by $x_{s}$. We fit for the amplitude of the oscillation. The top figure corresponds to perfect resolution for measuring $t$. The best fit for the amplitude is $0.29 \pm 0.04$. The lower figure corresponds to a resolution of $\sigma=0.2 \lambda$ which results in a factor of two dilution in the amplitude. The best fit value is $0.14 \pm 0.04$. Backgrounds (as discussed below) will also reduce the amplitude; none have been included in these histograms.

Tagging \& Reconstruction Efficiencies and Backgrounds

To observe an asymmetry, one $B_{s} / \bar{B}_{s}$ has to be tagged. Then the initial $\bar{B}_{s} / B_{s}$ nature of the other meson decaying to $\rho^{0} K_{S}^{0}$ is determined. The tagging of a $B_{s}$ can be achieved by either looking for a primary positively charged lepton or by reconstructing a $D_{s}^{-}$. The useful decay modes of the $D_{s}^{-}$ are $\phi \pi^{-}, \phi \pi^{+} \pi^{-} \pi^{-}$, and $K^{* 0} K^{-}$. Using only the $\phi$ decay to $K^{+} K^{-}(50 \%)$ and assuming an overall reconstruction efficiency of $60 \%$ for these final states, one arrives at a $D_{s}$ tagging efficiency of about $8 \%$. For the lepton tagging efficiency we assume the same value of $14 \%$ as in the $J / \psi K_{S}^{0}$ case above. With these numbers one can hope to achieve a total tagging efficiency of $20 \%$ for the $B_{s}$.

To investigate the reconstruction efficiency of the $\rho^{0} K_{S}^{0}$ final state, Monte Carlo $B_{s} \bar{B}_{s}$ events were generated at the $\Upsilon(5 \mathrm{~S})$ where one $B_{s}$ decayed to $\rho^{0} K_{S}^{0}$ and the other decayed to a representative mix of hadronic and semileptonic decays. This CP eigenstate was reconstructed by first searching for a $K_{S}^{0}$ using only the $\pi^{+} \pi^{-}$decay mode (neglecting $\pi^{0} \pi^{0}$ ), and then looking for another $\pi^{+} \pi^{-}$pair from a common vertex within the $\rho^{0}$ mass range. The four-vectors from the two decays were used to form an invariant $\rho^{0} K_{S}^{0}$ mass which for our Monte Carlo event sample shows a clear $B_{s}$ signal at 5.42 $\mathrm{GeV}$ over very little combinatorial background. The Monte Carlo simulation includes the detector effects as described in chapter 3. With rather loose cuts on the $K_{S}^{0}$ and $\rho^{0}$ masses one arrives at a detection efficiency of $32 \%$.

With these numbers one can estimate the integrated luminosity needed to obtain the 1000 events mentioned in the pre- 
vious subsection. Assuming that the $\Upsilon(5 \mathrm{~S})$ decays to $B_{s} \bar{B}_{s}$ (either directly or via $B_{s}^{*} \bar{B}_{s}^{*}$ ) $1 / 3$ of the time and that the energy spread of the machine is about the same as the width of the $\Upsilon(5 \mathrm{~S})$, one can start from a $B_{s} \bar{B}_{s}$ production cross section of $0.1 \mathrm{nb}$. Leaving the branching ratio to $\rho^{0} K_{S}^{0}$ as an unknown parameter, one needs $150 \mathrm{pb}^{-1} / \mathrm{BR}\left(B_{s} \rightarrow \rho^{0} K_{S}^{0}\right)$ in order to observe 1000 events. In other words, with a total integrated luminosity of $300 \mathrm{fb}^{-1}$ one can measure $\sin 2 \gamma$ if the branching ratio $\operatorname{BR}\left(B_{s} \rightarrow \rho^{0} K_{S}^{0}\right)$ is at least $5 \times 10^{-4}$.

The combinatorial background from the $B_{s} \bar{B}_{s}$ events themselves is small, about $2 \%$. Likewise, the background from $B_{u} \bar{B}_{u}$ and $B_{d} \bar{B}_{d}$ events on the $\Upsilon(5 \mathrm{~S})$ is small, about $4 \%$. The most serious background comes from the $q \bar{q}$ continuum events and can exceed several times the $B_{s} \rightarrow \rho^{0} K_{S}^{0}$ signal - increasing with decreasing $\rho^{0} K_{S}^{0}$ branching ratio. However, with tighter cuts on the $K_{S}^{0}$ and $\rho^{0}$ selection this background can be reduced by almost an order of magnitude - while decreasing the efficiency for the signal from $32 \%$ to $20 \%$. Probably the most powerful tool against this type of background is a multiple vertex fit, as described for $B \rightarrow \pi^{+} \pi^{-}$in Chapter 6 on rare decays.

Contamination from $\mathrm{C}=+1 B_{s} \bar{B}_{s}$ States

If the $B_{s} \bar{B}_{s}$ pair is produced in a $\mathrm{C}=-1$ state, the timedependent asymmetry is given by

$$
A(t)=\sin 2 \gamma \sin \left(x_{s} t / \tau\right)
$$

where $t$ is the time difference between decays as discussed above. If the $B_{s} \bar{B}_{s}$ pair is produced in a $\mathrm{C}=+1$ state (such as $e^{+} e^{-} \rightarrow B_{s} \bar{B}_{s}^{*} \rightarrow B_{s} \bar{B}_{s} \gamma$ ), then the analogous time-dependent asymmetry depends on the sum of the decay times. The time-dependent asymmetry for the time difference $t$ for the $\mathrm{C}=+1$ state takes the form

$$
A(t)=\sin 2 \gamma\left(\frac{x_{s}}{1+x_{s}^{2}} \cos \left(x_{s} t / \tau\right)+\frac{1}{1+x_{s}^{2}} \sin \left(x_{s} t / \tau\right)\right) .
$$

For large $x_{s}$, the $\cos \left(x_{s} t / \tau\right)$ term will dominate in this expression but will be supressed relative to the asymmetry in the 
The $B_{s} \bar{B}_{s}$ pairs in the $C=$ +1 state will introduce a small phase shift in the oscillation and dilute the asymmetry.

A large data sample above the $B_{s} \bar{B}_{s}$ threshold and an optimistically large branching fraction for $B_{s} \rightarrow \rho^{0} K_{S}^{0}$ are needed to measure $\sin 2 \gamma$.
$\mathrm{C}=-1$ state by a factor of $x_{s} /\left(1+x_{s}^{2}\right) \approx 1 / x_{s}$. The $\cos \left(x_{s} t / \tau\right)$ term will introduce a small phase shift in the oscillation. In addition, the contamination of these background events will dilute the asymmetry. The measured asymmetry $A_{\text {meas }}$ is related to the true asymmetry $A_{\text {true }}$ by the relation

$$
A_{\text {meas }}=A_{\text {true }}(1-P)
$$

where $P$ is the fraction of the total sample (including background) which is an independent background. Therefore, equal amounts of $\mathrm{C}=+1$ and $\mathrm{C}=-1$ states, for example, will dilute the asymmetry by a factor of two. ${ }^{3}$ The contamination must be small cnough so that the dilution is not significant and well enough known so that the dilution can be accurately estimated.

Conclusions on Measuring $\sin 2 \gamma$ Using $B_{s} \rightarrow \rho^{0} K_{S}^{0}$

For $\sin 2 \gamma \lesssim 0.3, x_{s} \gtrsim 3$, and no dilution of the asymmetry due to imperfect vertex resolution or backgrounds, the statistical error on $\sin 2 \gamma$ for a total of $N$ reconstructed, tagged events is approximately $\pi /(2 \sqrt{N})$. For $N=1000$, this gives an uncertainty of $\delta \sin 2 \gamma=0.05$. The reconstruciton efficiency for $B_{s} \rightarrow \rho^{0} K_{S}^{0}$ is estimated to be approximately $32 \%$. We estimate that the efficiency for tagging the other $B$ meson as a $B_{s}$ or $\bar{B}_{s}$ with a charged lepton or a fully reconstructed $D_{s}$ is about $20 \%$. Therefore, a reconstructed, tagged sample of $1000 B_{s} \rightarrow \rho^{0} K_{S}^{0}$ decays would require approximately $16,000 B_{s} \bar{B}_{s}$ events in which one of the $B$ 's decays to $\rho^{0} K_{S}^{0}$. To obtain such a sample with a total integrated luminosity of $300 \mathrm{fb}^{-1}$, the branching fraction for $B_{s} \rightarrow \rho^{0} K_{S}^{0}$ must be at least $5 \times 10^{-4}$. It may be quite a bit smaller than this; ${ }^{19}$ however, there are other CP eigenstates (e.g., $\omega K_{S}^{0}$ and $\omega K^{* 0}$, where $\omega \rightarrow \pi^{+} \pi^{-} \pi^{0}$ and $\left.K^{* 0} \rightarrow K_{S}^{0} \pi^{0}\right)$ which could be reconstructed with similar efficiency. Adding several such states together may give the statistical precision needed. The size of the asymmetry will be reduced by at least a factor of two (and hence the relative error will be increased by a factor of two) if the spatial resolution $\sigma$ divided by the spatial -wavelength of the oscillation $\lambda=2 \pi \beta \gamma c \tau / x_{s}$ is greater than 0.2 . For $\beta \gamma=1$ and $x_{s}=4 \pi$, for example, this corresponds to

\#3 The ratio of $\mathrm{C}=+1$ to $\mathrm{C}=-1$ states is not known. In the study of measuring $x_{s}$, it was assumed that at a center-of-mass energy above the threshold for $B_{s}^{*} \bar{B}_{s}^{*}$ production, the fraction of $B_{s} \bar{B}_{s}$ states which are $C=+1$ is 0.06 , which would only dilute the asymmetry by a factor of 0.94 . 
$33 \mu \mathrm{m}$ resolution for measuring the distance between decay vertices. The asymmetry will also be diluted due to decays to $\rho^{0} K_{S}^{0}$ from $B_{s}$ meson pairs in the $\mathrm{C}=+1$ state or from other backgrounds. The vertex resolution and background contamination must be well understood so that the expected dilution can be accurately estimated.

\subsection{Comparing Different Boosts and Beampipes}

In their study, Aleksan et $a l^{5}$. used a boost with $\beta \gamma \approx 1$ and assumed a beampipe radius of $1 \mathrm{~cm}$. It is important to determine how critical these parameters are, since machine design is simplified for a smaller boost, and such a small beampipe may not be achievable. Another study ${ }^{16}$ suggested that a boost with $\beta \gamma \approx 0.5$ would be closer to optimum.

We have conducted studies at several boosts to understand the advantages and disadvantages of a lower boost. Because we have assumed a constant geometrical acceptance in the laboratory frame, the reconstruction efficiency for $B \rightarrow$ $J / \psi K_{S}^{0}$ increases at lower boosts. Moreover, this efficiency is less sensitive to the low-angle cut-off, as is shown in Table 5.2 and Figure 5.6.

Table 5.2 Efficiency for reconstructing $B \rightarrow J / \psi K_{S}^{0}$ for various low-angle cut-offs.

\begin{tabular}{|c|c|c|}
\hline $\cos \theta$ & $\beta \gamma=0.56$ & $\beta \gamma=0.97$ \\
\hline 0.98 & $68 \%$ & $61 \%$ \\
\hline 0.95 & $56 \%$ & $48 \%$ \\
\hline 0.90 & $41 \%$ & $23 \%$ \\
\hline
\end{tabular}


Figure 5.6 The efficiency for reconstructing $B \rightarrow J / \psi K_{S}^{0}$ and tagging the other $B$, as a function of boost and low-angle cut-off. $E_{\text {high }}$ is the energy of higher energy beam.

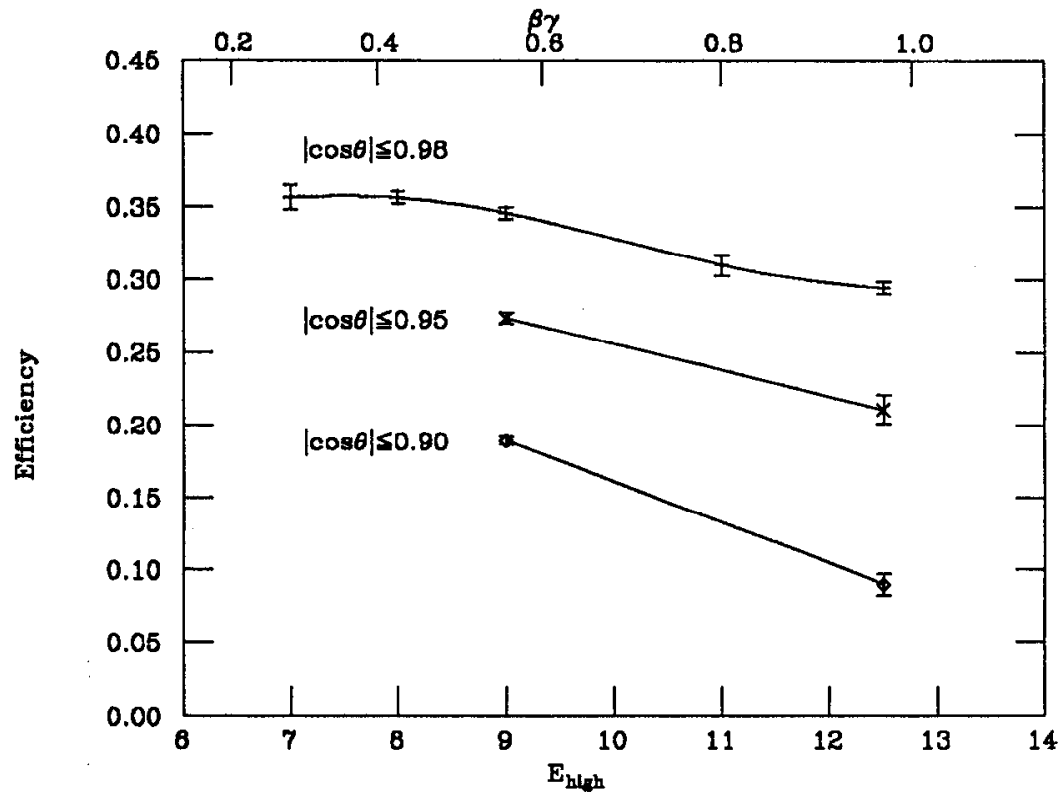

Our study also indicates that the resolution on $\Delta z$ improves somewhat going from $\beta \gamma=1$ to $\beta \gamma=0.56$, but the resolution scaled by $\beta \gamma c \tau$ (the mean $B$ vertex separation) gets worse. This is shown in Figure 5.7. The important criterion, however, is the error on measuring the $\mathrm{CP}$ violation amplitude, $\sin 2 \phi$. We have carried out identical analyses, as described in section 5.4, on samples of $20000 B_{d} \bar{B}_{d}$ events produced with three different boosts: $\beta \gamma=0.97(12.5 \mathrm{GeV}$ vs. $2.2 \mathrm{GeV}), \beta \gamma=0.56(9.0 \mathrm{GeV}$ vs. $3.1 \mathrm{GeV})$ and $\beta \gamma=0.43$ $(8.0 \mathrm{GeV}$ vs. $3.5 \mathrm{GeV})$. In all three cases, the error on $\sin 2 \beta$ is essentially the same; the results are summarized in Table 5.3. Thus any of these boosts is adequate for studying CP violation in $B_{d}$ mesons. 
Table 5.3 Error in measuring $\sin 2 \beta$ for different boosts and beampipe radii, with $300 \mathrm{fb}^{-1}$ of data.

\begin{tabular}{|c|c|c|}
\hline Boost $(\beta \gamma)$ & Beampipe Radius & Error on $\sin 2 \beta$ \\
\hline 0.97 & $1 \mathrm{~cm}$ & 0.025 \\
\hline 0.56 & $1 \mathrm{~cm}$ & 0.023 \\
\hline 0.43 & $1 \mathrm{~cm}$ & 0.023 \\
\hline 0.56 & $2 \mathrm{~cm}$ & 0.025 \\
\hline 0.43 & $2 \mathrm{~cm}$ & 0.025 \\
\hline
\end{tabular}

In order to evaluate whether a larger beampipe would provide adequate vertex resolution, we have conducted an identical study to the one described above (for the two smaller boosts) using a $2 \mathrm{~cm}$ radius beampipe in the simulation (see Figure 5.7). We find that the resolution on $\Delta z$ is degraded by only about $30 \%$. This leaves the error on $\sin 2 \beta$ essentially unchanged; these results are included in Table 5.3. Thus, according to this simulation (which includes a complete simulation of multiple scattering and nuclear interactions in the $1 \mathrm{~mm}$ Beryllium beampipe and two layers of $300 \mu \mathrm{m}$ thick silicon vertex detector), a $2 \mathrm{~cm}$ beampipe radius is acceptable. Of course, for other measurements - particularly any mixing or $\mathrm{CP}$ violation measurements involving $B_{s}$ mesons considerably more stringent demands are made on the vertex resolution.
The precision with which one can measure $C P$ violation in the $B_{d}$ system does not vary significantly for boosts in the range $\beta \gamma=0.43$ to 1.0 , and for beampipe radii in the range of 1 to $2 \mathrm{~cm}$. 
Figure 5.7 The resolution for measuring $\Delta z$, the distance between $B$ decays, scaled by the mean distance between the decay $(\beta \gamma c \tau)$ as a function of boost and beampipe radius.
In principle, $C P$ violation can be detected at a symmetric $B$ factory, if it is operating above the $\Upsilon(4 S)$.

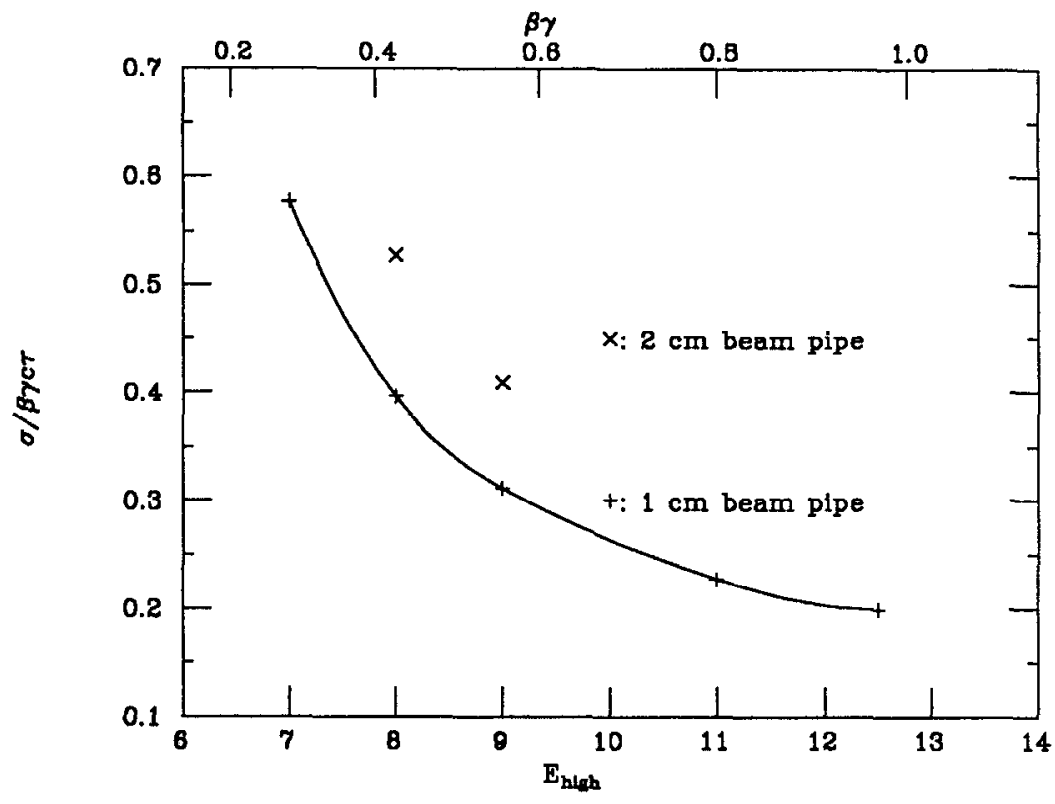

\subsection{Comparing Symmetric and Asymmetric $B$ FaC-} TORIES

We have outlined the methods and information required for measuring $\mathrm{CP}$ violation in the $B \bar{B}$ system at an asymmetric collider; however, this is not the only way this measurement could be made. In particular, a CP-violating asymmetry can be measured without any vertex information if the $B^{0}$ and $\bar{B}^{0}$ are in a $C=+1$ state. Such a state can be produced by running above $B \bar{B}^{*}$ threshhold; the $B^{*}$ decays to $B^{0} \gamma$.

When produced in a $C=+1$ state, the reaction rates for decays to a CP eigenstate and a tagging $\left(B^{0}\right.$ or $\left.\bar{B}^{0}\right)$ state are ${ }^{4}$

$$
\begin{aligned}
& f_{\mathrm{CP}}\left(t_{1}\right) f_{B}\left(t_{2}\right) \propto\left[1-\sin (2 \phi) \sin \left(\Delta m\left(t_{1}+t_{2}\right)\right)\right] e^{-\Gamma\left(t_{1}+t_{2}\right)}, \\
& f_{\mathrm{CP}}\left(t_{1}\right) f_{\bar{B}}\left(t_{2}\right) \propto\left[1+\sin (2 \phi) \sin \left(\Delta m\left(t_{1}+t_{2}\right)\right)\right] e^{-\Gamma\left(t_{1}+t_{2}\right)} . \# 4
\end{aligned}
$$

Note that they are symmetric in $t_{1}$ and $t_{2}$, in contrast to the equations in section 3.2. The time dependent asymmetry can

\#4 The sign convention we have used here is arbitrary; it depends on the $\mathrm{CP}$ of the eigenstate being reconstructed. 
be integrated over both $t_{1}$ and $t_{2}$ and results in

$$
A=\frac{2 x}{\left(1+x^{2}\right)^{2}} \sin 2 \phi .
$$

Although the dilution factor in front approaches $2 / x^{3}$ for large $x$ (as would be the case for $B_{s}$ mesons), it is approximately 0.6 for $x \approx 0.7$. Thus, even without any time measurement, an asymmetry can be measured: it would appear as an unequal number of $B$ and $\bar{B}$ tags detected in association with a CP eigenstate. This method has the advantage that the measurement can be made with a symmetric collider.

The 1988 Snowmass Summer Study group on $e^{+} e^{-} B$ factories concluded that asymmetric $B$ factory at the $\Upsilon(4 \mathrm{~S})$ had a factor of 4.7 advantage in the total luminosity needed to make a three standard deviation measurement of a CPviolating asymmetry for states with no background, compared to a symmetric $B$ factory operated just above $B \bar{B}^{*}$ threshhold. The Snowmass study assumed a total $B \bar{B}$ cross section of $1.2 \mathrm{nb}$ at the $\Upsilon(4 \mathrm{~S})$, of which $43 \%$ was assumed to be $B^{0} \bar{B}^{0}$ production. In contrast, above $B \bar{B}^{*}$ threshhold, the total $B \bar{B}$ cross section was estimated to be $0.3 \mathrm{nb}$, with $34 \%$ of this being $B_{d} \bar{B}_{d}^{*}+B_{d}^{*} \bar{B}_{d}$ production. This produces a factor of five advantage in useful cross section. In this backgroundfree study, therefore, this advantage arises primarily from the higher cross section at the $\Upsilon(4 S)$. In an actual experiment, however, the reduction in background afforded by the use of vertex cuts and by the higher intrinsic signal-to-background ratio at the $\Upsilon(4 S)$ are equally important.

The symmetric case has a small advantage in the "dilution" factor $d$ (see Chapter 2.5), which relates the measurable asymmetry $\mathrm{A}$ to $\sin 2 \beta$ : a smaller value of $d$ requires a larger number of reconstructed and tagged events to measure $\sin 2 \beta$ to a given precision. For the symmetric collider case, $d$ is just the fully time-integrated dilution, $2 x /\left(1+x^{2}\right)^{2}$, which equals 0.63 for $x=0.71$ (neglecting a factor of $\sqrt{1-A^{2}}$ ). The integrated asymmetry for the asymmetric collider case is $d=x /\left(1+x^{2}\right)=0.47$ for $x=0.71$. The fitting method described here, however, yields a larger value of $d$. The uncertainty due to the number of correct tags derived from the fit
A symmetric $B$ factory would require at least five times more luminosity to achieve the same level of precision in measuring $C P$ violation, compared to an asymmetric $B$ factory. 
Because the CP-violation measurement at a symmetric factory must be done above the $\Upsilon(4 S)$, where the $B$ cross section is a factor of four smaller, all other $B$ physics studies suffer. is 0.0208 for $N=6914$ events; the effective value is therefore $d=0.58$, slightly smaller than the value of 0.61 used in reference 4 , but larger than the fully time-integrated value. (Note that the error quoted in section 5.4 is larger, as it includes, in addition, the error due to the correction for wrong-sign tags, which we assume are the same for both the symmetric and asymmetric cases.)

Since new measurements by $\mathrm{CLEO}^{20}$ indicate that $B^{0} \bar{B}^{0}$ production is $\sim 50 \%$ (rather than $43 \%$ ) of the $\Upsilon(4 \mathrm{~S}$ ) cross section, the luminosity advantage of the asymmetric collider has actually increased from 4.7 to 5.4 . It is important in the design of a high-luminosity asymmetric collider at the $\Upsilon(4 \mathrm{~S})$ to pay attention to the center-of-mass energy spread, as this reduces the effective $\Upsilon(4 \mathrm{~S})$ cross section, and could thereby reduce the luminosity advantage.

What other advantages does an asymmetric collider offer compared to a symmetric machine? Perhaps the foremost is that it allows the CP violation study to be done at the $\Upsilon(4 S)$, where the cross section is maximized for all the other $B_{d}$ and $B_{u}$ physics one wants to do. For a symmetric $B$ factory running off the $\Upsilon(4 \mathrm{~S})$, all the other physics studies will suffer from the reduced cross section. Also, on the $\Upsilon(4 \mathrm{~S})$ the continuum cross section is only a factor of three larger than the $B \bar{B}$ cross section and, as we shall show, the vertex information at an asymmetric collider can be used to suppress a very large fraction of this background. Above the $\Upsilon(4 S)$ the continuum generates a much larger relative background; a large amount of continuum data must be taken below the $\Upsilon(4 \mathrm{~S})$ in order to perform a background subtraction, taking more time and integrated luminosity away from the $B$ physics program.

Above the $\Upsilon(4 \mathrm{~S}), B^{0} \bar{B}^{0}$ production may represent 10 to $20 \%$ of the $B \bar{B}$ cross section; since these $B^{0} \bar{B}^{0}$ pairs will be produced in a $C=-1$ state, care must be taken to distinguish these events from the $B \bar{B}^{*}$ events in order to prevent a sizeable dilution of the $C=+1 \mathrm{CP}$ asymmetry measurment. This can be done given sufficient mass resolution.

Another important difference is the "confidence" one can place in the CP-asymmetry measurements. In our method, 
we have important internal cross checks: the total number of events with a $B^{0}$ tag should equal the total number of events with a $\bar{B}^{0} \mathrm{tag}$; the total number of events in which the CP eigenstate decay precedes the tagging decay should equal the total number of events in which the CP eigenstate decay follows the tagging decay. Only when the events are sorted into the proper four categories, and then properly combined (see section 3.2 ) will the asymmetry appear. Then the asymmetry should be clearly seen in deviations from exponential decay rates. When measuring a time integrated asymmetry with $B \bar{B}^{*}$ events, one must rely entirely on an asymmetry of the tags; every other possible source of such an asymmetry must be ruled out in some way.

Finally, we will show (in Chapter 6) that the vertexing information available at an asymmetric collider is very useful in suppressing continuum background to the reaction $B \rightarrow \pi^{+} \pi^{-}$. Measuring $\sin 2 \alpha$ at a symmetric machine may be much more difficult than measuring $\sin 2 \beta$. While it is clear that it will require a great deal of luminosity $(\sim 300$ $\mathrm{fb}^{-1}$ ) and good vertex resolution at an asymmetric collider, we know of no reason it cannot be done. And although measuring $\sin 2 \gamma$ will require a great deal of luck (along with a large data sample) at an asymmetic $B$ factory, it is completely impossible at a symmetric collider since the integration dilution goes as $\sim 2 / x^{3}$; this is clearly too high a price to pay when $x_{s} \gtrsim 3$.
The time-dependent method used at an asymmetric $B$ factory allows important cross checks. 


\section{REFERENCES}

1. S. L. Wu, Proceedings of the 1987 International Symposium on Lepton and Photon Interactions at High Energies, ed. by W. Bartel and R. Ruckl, p. 39 (1988).

2. H. Albrecht et al., Phys. Lett. 192B, 245 (1987). This paper by the Argus collaboration presents a result of $r=0.21 \pm 0.08$, where $r$ is the mixing parameter.

3. M. Artuso et al., Phys. Rev. Lett. 62, 2233 (1989). The CLEO collaboration reports $r=0.19 \pm 0.06 \pm 0.06$.

4. G. Feldman et al., SLAC-PUB-4838 (1988); to be published in the Proceedings of the 1988 Summer Study on High Energy Physics in the 1990s, Snowmass, Colorado, 1988.

5. Our report here on the $J / \psi K_{S}^{0}$ eigenstate is largely based on the published study R. Aleksan, J. Bartelt, P. R. Burchat, A. Seiden, Phys. Rev. D39, 1283 (1989); more details of the Monte Carlo and analysis procedure can be found there.

6. P. Oddone, Proceedings of the UCLA Workshop: Linear Collider $B \bar{B}$ Factory Conceptual Design, ed. by D. Stork, p. 243 (1987).

7. I.I. Bigi and A.I. Sanda, Nucl. Phys. B193, 85 (1981).

8. L. Wolfenstein, Nucl. Phys. B246, 45 (1984); M.B. Gavela et al., Phys. Lett. 162B, 197 (1985).

9. I. Dunietz and J.L. Rosner, Phys. Rev. D34, 1404 (1986).

10. I.I. Bigi and A.I. Sanda, Nucl. Phys. B281, 41 (1987).

11. I.I. Bigi and A.I. Sanda, Phys. Lett. 194B, 307 (1987).

12. R. Brun et al.CERN DD/EE/84-1 (1987).

13. Rcview of Particle Properties, Phys. Lett. 170B (1987).

14. See, for example, D.G.Hitlin, CALT-68-1463 (1987).

15. M. S. Alam et al., Phys. Rev. D34, 3279 (1986); H. Albrecht et al., Phys. Lell. 199B, 451 (1987):-

16. D. Hitlin, T. Nakada and A. Sanda, Proceedings of the 1988 Summer Study on High Energy Physics in the 1990s, Snowmass, Colorado, 1988. 
17. R. Aleksan, Minutes of $B \bar{B}$ Working Group Meeting, 20 April 1988, SLAC, unpublished.

18. M. Bauer, B. Stech and M. Wirbel, Z. Phys. C34, 103 (1987).

19. From Bauer, Stech and Wirbel's calculation of $B_{d} \rightarrow$ $\rho^{0} \pi^{0^{18}}$ a branching ratio for $B_{s} \rightarrow \rho^{0} K_{S}^{0}$ of $\sim 10^{-6}$ is plausible.

20. For example, the talk presented by David L. Kreinick for CLEO at the 14th International Symposium on Lepton and Photon Interactions, Stanford, 1989; to be published. 


\section{B DECAY PHySICS OTHER THAN CP VIOLATION}

\subsection{INTRODUCTION}

$\mathrm{T}$ he measurement of $\mathrm{CP}$-violation in the $B$ system is the primary goal of a next-generation $B$ factory. Along the way, however, a spectacular bounty of physics results can be reaped at lower levels of integrated luminosity. The $B$ system presents a unique opportunity to measure basic parameters of the Standard Model and to probe for heavy objects which can manifest themselves in decays which proceed via loop diagrams. In addition, since $B$ decays are expected to be dominated by short-distance dynamics, they provide an important testing ground for perturbative QCD. It is difficult to overestimate the richness of the physics program which awaits the next high-luminosity $B$ factory.

The $B$ system has only begun to be explored; less than $10 \%$ of the exclusive decay modes have been measured and only a few hundred $B$ decays have been fully reconstructed. CLEO, and to a lesser extent ARGUS, can be expected to dominate the field for the next five years. CESR has achieved a peak luminosity of $10^{32} \mathrm{~cm}^{-2} \mathrm{sec}^{-1}$ and expects to improve this by a significant factor; the upgraded CLEO II detector has greatly improved electromagnetic calorimetry allowing, modes with neutrals to be reconstructed with greater efficiency. SLC and LEP will also producc a substantial number of $B$ 's, and although fewer in number than CESR these will be moving $B$ 's, which allows secondary vertices to be reconstructed. Despite the advances expected in the field of $B$ physics in the next few years, there are many interesting topics, in addition to $\mathrm{CP}$-violation, which are likcly to remain out of reach or to be only partially explored. This is because no one machine will have the combined advantages of high luminosity, high cross-section on the $\Upsilon(4 \mathrm{~S})$, and moving $B$ 's.

We present in this chapter several detailed analyses showing how the advantages of a high-luminosity, asymmetric machine can be exploited in order to make significant inroads in $B$ decay physics. The analyses are divided into the following categories: 
1. $B_{s}$ mixing.

2. Exclusive $b \rightarrow c$ hadronic decays.

3. Exclusive $b \rightarrow c$ semi-leptonic decays.

4. Exclusive $b \rightarrow u$ decays.

5. Penguin diagrams.

6. Annihilation diagrams.

In all but the last category, which is extremely difficult at any machine, the analyses indicate that an asymmetric machine operating at an initial luminosity of above $10^{33} \mathrm{~cm}^{-2} \mathrm{sec}^{-1}$ will begin to produce significant new physics results in the first year.

\section{2. $B_{s} \bar{B}_{s}$ MIXING}

\section{Introduction and Formalism}

Just as the $K^{0}$ and $\bar{K}^{0} \mathrm{mix}$ to produce mass eigenstates $K_{S}^{0}$ and $K_{L}^{0}$, neutral $B$ meson mass eigenstates should be a mixture of $B$ and $\bar{B}$. In particular, measurements by ARGUS ${ }^{1}$ and $\mathrm{CLEO}^{2}$ have reported a high degree of mixing between the $B_{d}$ and $\bar{B}_{d}$ mesons, which leads to predictions that mixing of $B_{s} \bar{B}_{s}$ mesons should be "maximal". The mixing (in both cases) comes about through box diagrams involving $W^{ \pm}$ and heavy quarks, primarily, the top quark. The mixing can be described by a mass matrix with non-zero off-diagonal elements:

$$
\left[\begin{array}{cc}
M-\frac{1}{2} i \Gamma & M_{12}-\frac{1}{2} i \Gamma_{12} \\
M_{12}^{*}-\frac{1}{2} i \Gamma_{12}^{*} & M-\frac{1}{2} i \Gamma
\end{array}\right] .
$$

By diagonalizing this matrix we obtain $B_{1}$ and $B_{2}$, the mass eigenstates with definite widths; $M$ and $\Gamma$ are the average Inass and width. If we define

$$
Q \equiv \sqrt{\left(M_{12}-\frac{1}{2} i \Gamma_{12}\right)\left(M_{12}^{*}-\frac{1}{2} i \Gamma_{12}^{*}\right)}
$$

then $\Delta m=2 \operatorname{Re} Q$ and $\Delta \Gamma=-4 \operatorname{Im} Q$. For the $B$ system, $\Delta m \gg$ $\Delta \Gamma$, so that the lifetime difference for $B$ 's is small, in contrast to the $K^{0}$ system.
Mixing of $B_{s} \bar{B}_{s}$ mesons is predicted to be "maximal". 
The $B_{s}$ mixing parameter $x_{s}$ is expected to be $Z 3$ and could be much larger

For large $x_{s}$, only a timedependent method can be used.
Defining $x \equiv \Delta m / \Gamma$ and $y \equiv \Delta \Gamma / 2 \Gamma$, the ratio $r$ measured by ARGUS and CLEO is

$$
r=\frac{P\left(B^{0} \rightarrow \bar{B}^{0}\right)}{P\left(B^{0} \rightarrow B^{0}\right)}=\frac{x^{2}+y^{2}}{2+x^{2}-y^{2}} \approx \frac{x^{2}}{2+x^{2}},
$$

since $x \gg y$. Because of the dominance of the top quark in the box diagrams (assuming that there are only three generations), $x$ can be written

$$
x_{q}=\frac{G_{F}^{2}}{6 \pi^{2}} m_{t}^{2} \tau_{B_{q}} B_{B_{q}} f_{B_{q}}^{2} m_{B_{q}}\left|V_{t q} V_{t b}^{*}\right|^{2} \frac{A\left(z_{t}\right)}{z_{t}} \eta_{t}
$$

where $q$ stands for $d$ or $s$. Here, $G_{F}$ is the Fermi constant, $\tau_{B_{q}}$ and $m_{B_{q}}$ are the appropriate $B$ meson lifetime and mass, and $B_{B_{q}}$ and $f_{B_{q}}$ are the $B$ meson "bag" constant and decay constant. $V_{t q}$ and $V_{t b}^{*}$ are the relevant Kobayashi-Maskawa matrix elements; $\eta_{t}$ is the QCD correction factor of order one $\left(\approx 0.85^{3}\right) . A\left(z_{t}\right) / z_{t}$, where $z_{t} \equiv m_{t}^{2} / m_{W}^{2}$, is a slowly varying function of the top quark mass; it equals 0.75 for $m_{t}=m_{W}$ and drops to 0.52 for $m_{t}=200 \mathrm{GeV}$.

Most of these factors cancel, at least approximately, in the ratio $x_{s} / x_{d}$; we are left with

$$
\frac{x_{s}}{x_{d}} \approx \frac{\left|V_{t s}\right|^{2}}{\left|V_{t d}\right|^{2}}
$$

Existing measurements constrain this ratio to be equal to or greater than 6 . Averaging the results of ARGUS and CLEO gives $x_{d}=0.71_{-0.14}^{+0.13}$; this implies that $x_{s} \gtrsim 3$. It could be considerably larger; $x_{s} \approx 25$ is not ruled out. If $x_{s} \lesssim 2$, this would be evidence for a fourth generation of quarks mixing with the known three generations.

We have carried out a Monte Carlo study to determine the limits of measuring $x_{s}\left(\Delta m / \Gamma\right.$ for the $\left.B_{s}\right)$ at an asymmetric $B$ factory. ARGUS and CLEO have measured the ratio of like-sign dilepton events to opposite-sign dilepton events, for $B_{d} \bar{B}_{d}$ events. For $B_{s} \bar{B}_{s}$ events this ratio approaches 1 as $x_{s}$ increases, and loses all sensitivity for determining $x_{s}$ if $x_{s} \gtrsim 5$, given a $10 \%$ measurement of the ratio. Thus a time-dependent method, as opposed to this time-integrated method, is required. 
The goal of the study was to find the largest value of $x_{s}$ ( $\equiv x_{\max }$ ) which could be measured for a given boost, vertex resolution and integrated luminosity at an asymmetric collider, in order to gain some understanding of the possible trade-offs in these parameters. The method is to use like-sign dilepton events to determine the high frequency modulation in the $\Delta z$ distribution caused by the rapid time oscillation in the $B_{s}$ mixing. Here $\Delta z$ is the distance between the leptons along the beamline, measured where the tracks cross in the $x-y$ plane. The assumptions we have made in generating "events" will be outlined below; our methods for extracting $x_{s}$ will then be described; we end this section with our conclusions.

For $B_{s}$ meson pairs produced in a $C=-1$ state, i.e., $B_{s} \bar{B}_{s}$ and $B_{s}^{*} \bar{B}_{s}^{*}$ pairs, the mixing depends only on the time difference of the decays $\left(\Delta t \equiv t_{2}-t_{1}\right)$. Because the $\Upsilon(4 S)$ is below threshold for $B_{s} \bar{B}_{s}$ and $B_{s}^{*} \bar{B}_{s}^{*}$ pairs, we must work above it, preferably at the $\Upsilon(5 S)$. The quantity $\Delta t$ is observable, neglecting the small momentum of the $B$ mesons in the $\Upsilon(5 \mathrm{~S})$ center-of-mass frame: $\Delta t \approx \Delta z /(\beta \gamma c)$, where $\beta \gamma$ is $p / m$ for the $\Upsilon(5 S)$ in the laboratory frame. Because of the large size of the beams (relative to the decay lengths) the individual times $t_{1}$ and $t_{2}$ are not measurable and are not required. In effect, we can integrate over $t_{1}$ with no loss of information. For $B_{s}$ meson pairs produced in a $C=+1$ state ,i.e., $B_{s} \bar{B}_{s}^{*}$, the mixing depends on the sum $t_{1}+t_{2}$. We can replace $t_{2}$ by $t_{1}+\Delta t$ and then integrate over $t_{1}$. The resulting dependence on $\Delta t$ could, in principle, be observed, but because it has a small amplitude on top of a large purely exponential component, it is much less distinct than in the $C=-1$ case. It will be treated only as a background in this study.

\section{Input Assumptions}

For the total production cross section and relative rates for various combinations of $B$ mesons, we have used the CUSB data and analysis presented in $1988 .^{4}$ Because these data show the cross section for $B \bar{B}$ production dipping to zero in the range 10.750 to $10.800 \mathrm{GeV}$ (the narrow window above $B_{s} \bar{B}_{s}$ threshold, but below $B_{s} \bar{B}_{s}^{*}$ ), we have chosen not to work at
To produce $B$, meson pairs in a $C=-1$ state we would work at the $\Upsilon(5 \mathrm{~S})$. 
We have assumed that the $\Upsilon(5 S)$ is above $B_{s}^{*} \bar{B}_{s}^{*}$ threshhold. this energy, but rather at the $\Upsilon(5 S)$. The total cross section for $B$ meson production is taken to be $0.27 \mathrm{nb}$ at the $\Upsilon(5 \mathrm{~S})$ peak.

In addition we have assumed that in all cases , $B \bar{B}, B \bar{B}^{*}$, and $B^{*} \bar{B}^{*}$, the relative proportions of $B_{u} \bar{B}_{u}$ to $B_{d} \bar{B}_{d}$ are equal, as they are, at least approximately, at the $\Upsilon(4 \mathrm{~S})$. Table 6.1 lists the proportions of event types. Note that CUSB's model has the peak of the $\Upsilon(5 \mathrm{~S})$ above $B_{s}^{*} \bar{B}_{s}^{*}$ threshold; this is an optimistic assumption for this study. If $B_{s}^{*} \bar{B}_{s}^{*}$ is not produced at the $\Upsilon(5 \mathrm{~S})$, or is produced at a much reduced rate, it may be necessary to go to a higher energy, since this is the primary source of the signal. We have also followed the CUSB analysis and assumed that three-body decays of the $\Upsilon(5 \mathrm{~S})\left(\right.$ e.g., $\left.B^{+} \bar{B}^{0} \pi^{-}\right)$are negligible.

Table 6.1 Relative Rates Assumed for $B$ Species

\begin{tabular}{|c|c|c|}
\hline Species & Fractional Rate & Type of mixing \\
\hline$B_{u}^{*} \bar{B}_{u}^{*}$ & 0.11 & no mixing \\
\hline$B_{d}^{*} \bar{B}_{d}^{*}$ & 0.11 & $C=-1 x_{d}$ mixing \\
\hline$B_{s}^{*} \bar{B}_{s}^{*}$ & 0.26 & $C=-1 x_{s}$ mixing \\
\hline$B_{u} \bar{B}_{u}^{*}$ & 0.15 & no mixing \\
\hline$B_{d} \bar{B}_{d}^{*}$ & 0.15 & $C=+1 x_{d}$ mixing \\
\hline$B_{s} \bar{B}_{s}^{*}$ & 0.02 & $C=+1 x_{s}$ mixing \\
\hline$B_{u} \bar{B}_{u}$ & 0.07 & no mixing \\
\hline$B_{d} \bar{B}_{d}$ & 0.07 & $C=-1 x_{d}$ mixing \\
\hline$B_{s} \bar{B}_{s}$ & 0.05 & $C=-1 x_{s}$ mixing \\
\hline
\end{tabular}

For decay rates, we have assumed that all $B$ mesons have the same lifetime, $1.1 \mathrm{ps}$. This is smaller than the $1.3 \mathrm{ps} 1988$ Particle Data Group value, but closer to the value of $1.15 \pm$ 0.14 reported at the International Conference on High Energy Physics in Munich, August, $1988^{6}$. We have also taken the semileptonic branching ratios to be $10 \%$ each for electrons and muons, as reported at Munich. The efficiency for detecting each of these leptons was taken to be $50 \%$, independent of 
the boost. 'Thus, multiplying these factors together, $1 \%$ of all $B \bar{B}$ events are included in the "data sample".

Rather than actually doing detector simulations for tens or hundreds of thousands of events, we have simply generated the $\Delta z$ according to the appropriate formulae, and then applied Gaussian smearing. More complete simulations show a definite non-Gaussian tail to the $\Delta z$ resolution, but for simplicity's sake we have ignored this. We have also ignored the small, but non-zero, momenta of the $B$ mesons in the centerof-mass rest frame. These momenta will also effectively add a small amount to the smearing when relating $\Delta z$ to $\Delta t$ : about $20 \mu \mathrm{m}$ on average, for $B_{s}$ mesons coming from $B_{s}^{*} \bar{B}_{s}^{*}$ events (the type of event which produces most of the signal), when $\beta \gamma \approx 1$. Thus, when a resolution of $40 \mu \mathrm{m}$ is used in this study, it should be considered to be comprised of $20 \mu \mathrm{m}$ smearing due to momenta plus $35 \mu \mathrm{m}$ smearing from detector resolution, added in quadrature to yield an effective resolution of $40 \mu \mathrm{m}$.

Mixing formulae for the different cases are shown below (these equations were found in Krawczyk et al. ${ }^{7}$ but are presented here with slight changes in notation). Of course, for $B_{u} \bar{B}_{u}$ events, there is no mixing, and no contribution to the like-sign event distribution, only a purely exponential contribution to the opposite-sign distribution. For $C=-1$ mixing events $\left(B_{d} \bar{B}_{d}, B_{d}^{*} \bar{B}_{d}^{*}, B_{s} \bar{B}_{s}\right.$ and $\left.B_{s}^{*} \bar{B}_{s}^{*}\right)$ the opposite-sign and like-sign events are distributed respectively as:

$$
\begin{aligned}
& \frac{1}{\tau^{2}} \exp \left(-\frac{t_{1}+t_{2}}{\tau}\right) \cos ^{2}\left(\frac{x}{2} \frac{\Delta t}{\tau}\right) \\
& \frac{1}{\tau^{2}} \exp \left(-\frac{t_{1}+t_{2}}{\tau}\right) \sin ^{2}\left(\frac{x}{2} \frac{\Delta t}{\tau}\right)
\end{aligned}
$$

where $x$ is $x_{s}$ for $B_{s}$ events and $x_{d}$ for $B_{d}$ events; $x_{d} \equiv 0.70$ for this study; $\tau$ is the mean $B$ lifetime quoted above. After substituting $t_{1}+\Delta t$ for $t_{2}$, where $t_{2} \geq t_{1}$, and integrating over $t_{1}$, the formulae are:

$$
\frac{1}{\tau} \exp \left(-\frac{\Delta t}{\tau}\right) \cos ^{2}\left(\frac{x}{2} \frac{\Delta t}{\tau}\right) \quad \frac{1}{\tau} \exp \left(-\frac{\Delta t}{\tau}\right) \sin ^{2}\left(\frac{x}{2} \frac{\Delta t}{\tau}\right),
$$

The simple time dependence is thus preserved.
We have generated decay lengths and applied Gaussian smearing; no real kinematics or detector simulation was done. 
For $B_{d} \bar{B}_{d}^{*}$ and $B_{s} \bar{B}_{s}^{*}$ events $(C=+1)$ the opposite-sign and like-sign formulae are:

$$
\begin{aligned}
& \frac{1}{\tau^{2}} \exp \left(-\frac{t_{1}+t_{2}}{\tau}\right) \cos ^{2}\left(\frac{x}{2} \frac{t_{1}+t_{2}}{\tau}\right) \\
& \frac{1}{\tau^{2}} \exp \left(-\frac{t_{1}+t_{2}}{\tau}\right) \sin ^{2}\left(\frac{x}{2} \frac{t_{1}+t_{2}}{\tau}\right) .
\end{aligned}
$$

After substituting and integrating as before, the relations take on the form:

$$
\begin{aligned}
& \frac{1}{2 \tau\left(1+x^{2}\right)} \exp \left(-\frac{\Delta t}{\tau}\right)\left[2 \cos ^{2}\left(\frac{x}{2} \frac{\Delta t}{\tau}\right)+x^{2}-x \sin \left(x \frac{\Delta t}{\tau}\right)\right] \\
& \frac{1}{2 \tau\left(1+x^{2}\right)} \exp \left(-\frac{\Delta t}{\tau}\right)\left[2 \sin ^{2}\left(\frac{x}{2} \frac{\Delta t}{\tau}\right)+x^{2}-x \sin \left(x \frac{\Delta t}{\tau}\right)\right]
\end{aligned}
$$

No backgrounds from the continuum or from cascade decays were included.

To generate the $\Delta z$ distributions, we simply substituted $\Delta z /\langle\Delta z\rangle$ for $\Delta t / \tau$ (where $\langle\Delta z\rangle \equiv \beta \gamma c \tau$ ), and then applied Gaussian smearing, to approximate both the vertex resolution and the small momenta of the $B$ mesons in the $\Upsilon(5 \mathrm{~S})$ rest frame.

We have not included backgrounds from $c \bar{c}$ events, or from semileptonic decays of charmed mesons from the $B$ mesons, or, indeed, from any source. We assume that most of these backgrounds can be eliminated by cuts on the leptons' momenta. For $c \bar{c}$ events there is no large source of like-sign events, because $D \bar{D}$ mixing is known to be small; and for most cascade events, which could produce like-sign lepton backgrounds, there would be no rapid oscillation in the background. There is one obvious exception to this: if leptons from the $B_{s} \rightarrow D X \rightarrow \ell X$ cascade are misidentified as having come directly from the $B_{s}$ meson, this could create like-sign events with a rapid time oscillation, smeared by the $D$ lifetime. If this background cannot be eliminated by momentum cuts, etc., it could make the measurement more difficult. 


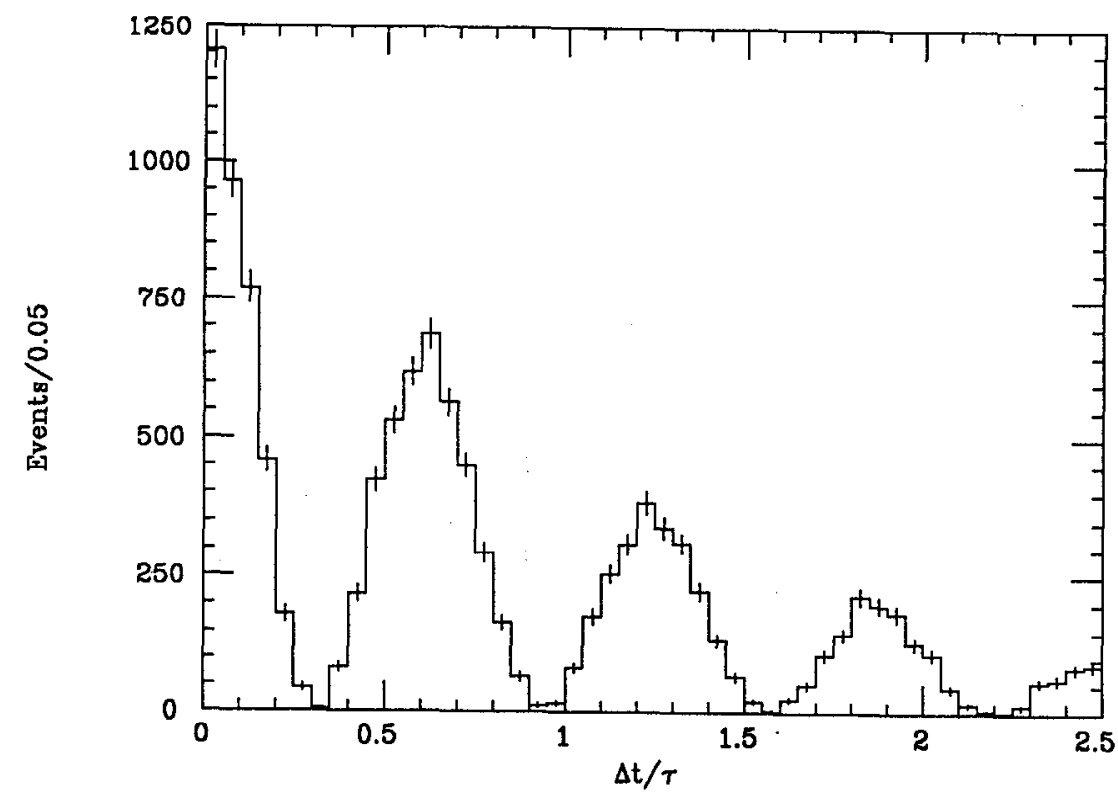

Figure 6.1 a\&b The (unsmeared) decay time distribution for $C=-1 B_{s}$ meson pairs; the upper plot is for opposite-sign lepton events, the lower for like-sign lepton events. This represents $30 \mathrm{fb}^{-1}$ of data, with $x_{s}=10$.

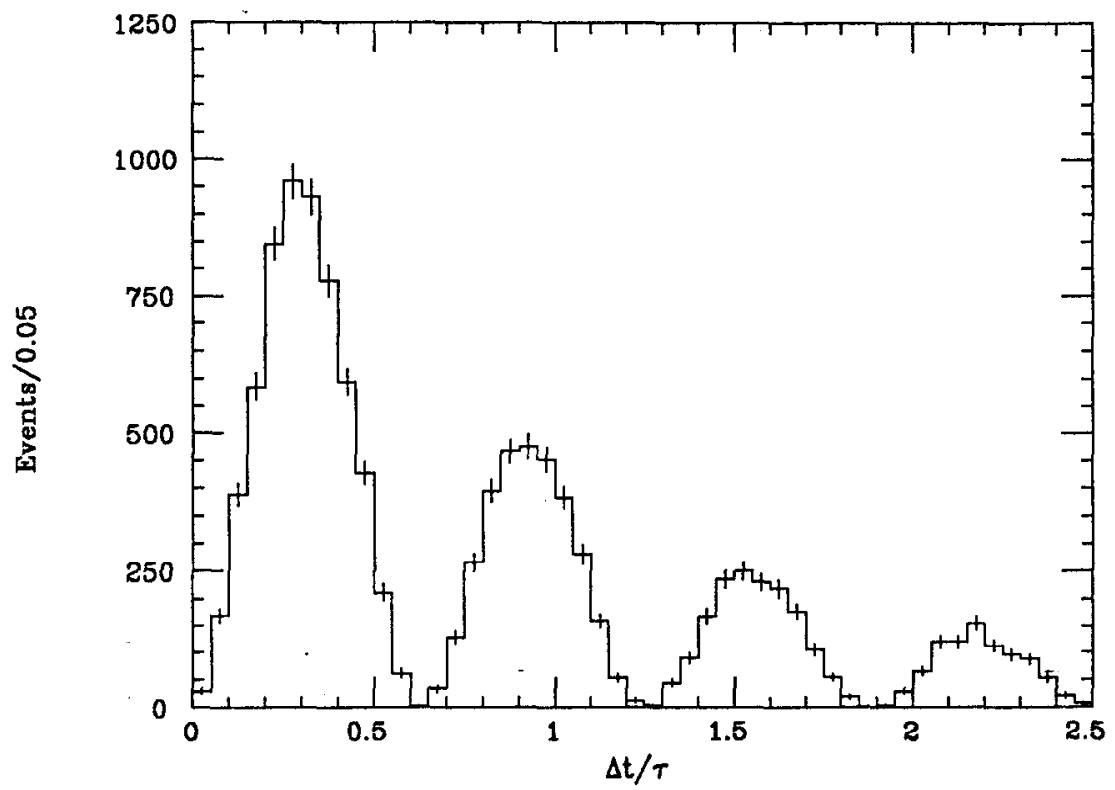


Figure 6.2 a\&b The same data as in Figure 6.1, but now smeared by $\sigma=0.25\langle\Delta z\rangle$.
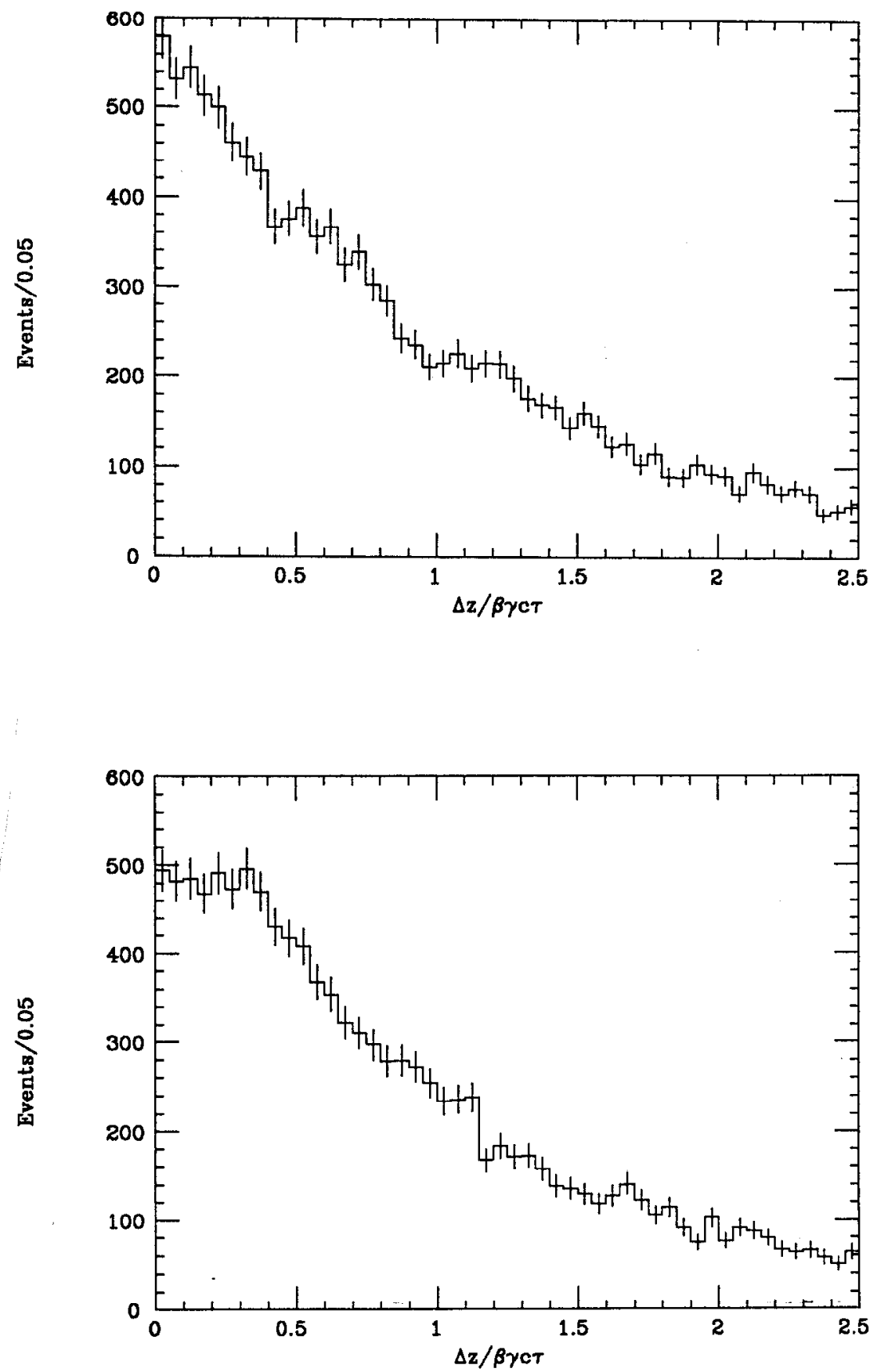

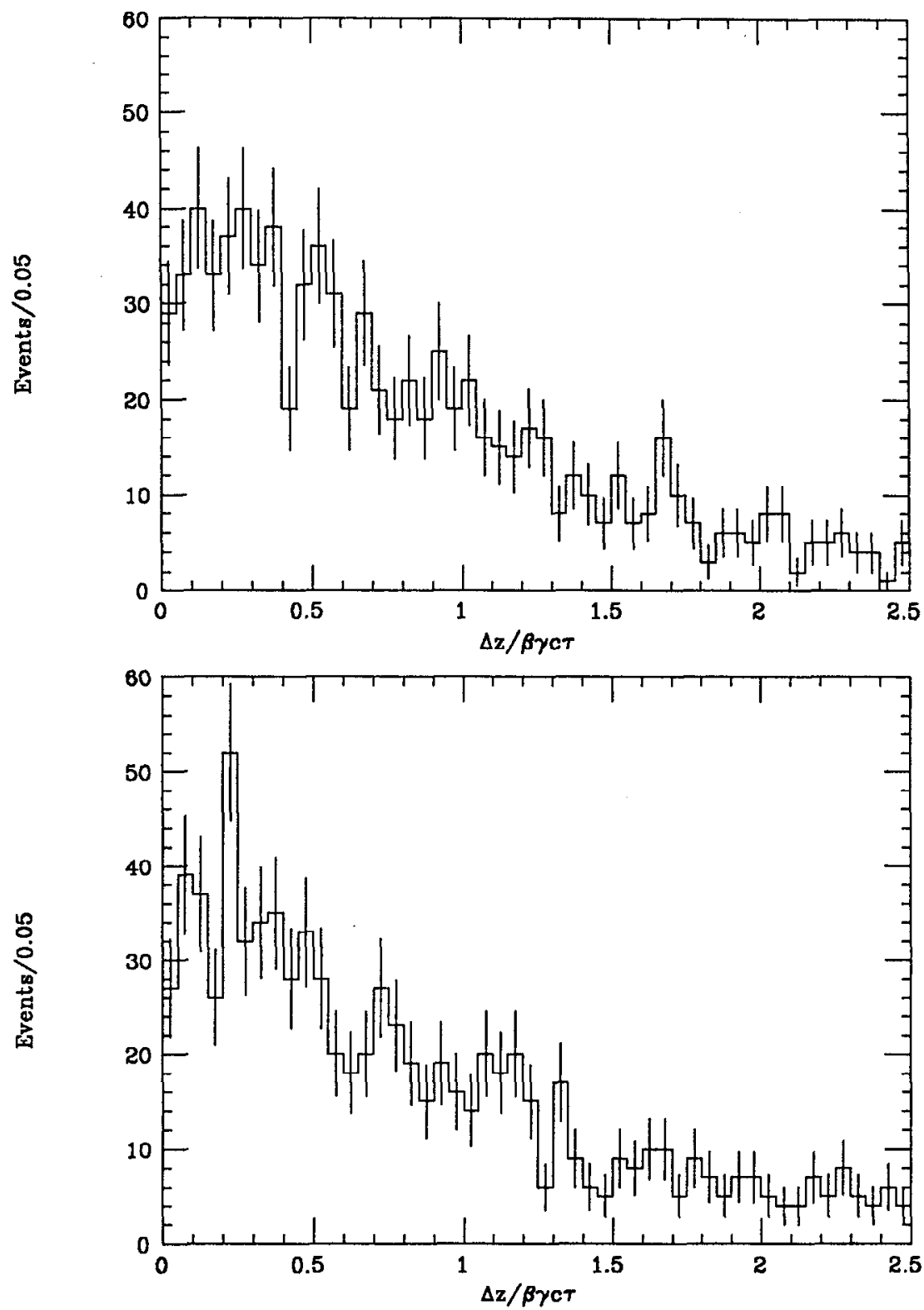

The only unit of length in the study is the average $B$ decay length $\langle\Delta z\rangle$. Thus the resolution, $\sigma$, is measured in units of $\langle\Delta z\rangle$. Seven values were chosen for study: $0.667,0.444$, $0.333,0.250,0.182,0.143$, and 0.125 (reciprocally, these correspond to $\langle\Delta z\rangle / \sigma=1.5,2.25,3,4,5.5,7$ and 8 , respectively). To achieve a resolution as small as $0.125\langle\Delta z\rangle$ would require a high boost machine $(12.5 \mathrm{GeV} / \mathrm{c}$ vs. $2.2 \mathrm{GeV} / \mathrm{c}$, so that $\langle\Delta z\rangle=320 \mu \mathrm{m})$ and excellent vertex resolution $(40 \mu \mathrm{m})$. A machine with a small asymmetry, e.g., $7 \mathrm{GeV} / \mathrm{c}$ vs. $4 \mathrm{GeV} / \mathrm{c}$,
Figure 6.3 a\&b The (smeared) decay path length distribution for $C=+1 B$, meson pairs; the upper plot is for opposite-sign lepton events, the lower for like-sign lepton events. This represents $30 \mathrm{fb}^{-1}$ of data, with $x_{s}=10$ and $\sigma=0.25\langle\Delta z\rangle$. 
Figure 6.4 a\&b The (smeared) decay path length distribution for $C=-1 \quad B_{d}$ meson pairs; the upper plot is for opposite-sign lepton events, the lower for like-sign lepton events. This represents $30 \mathrm{fb}^{-1}$ of data, with $x_{d}=0.70$ and $\sigma=0.25\langle\Delta z\rangle$. with $\langle\Delta z\rangle=90 \mu \mathrm{m}$, and a resolution of $60 \mu \mathrm{m}$ would fall at the other end. The study has been carried out at two different values of integrated luminosity: 3 and $30 \mathrm{fb}^{-1}$.
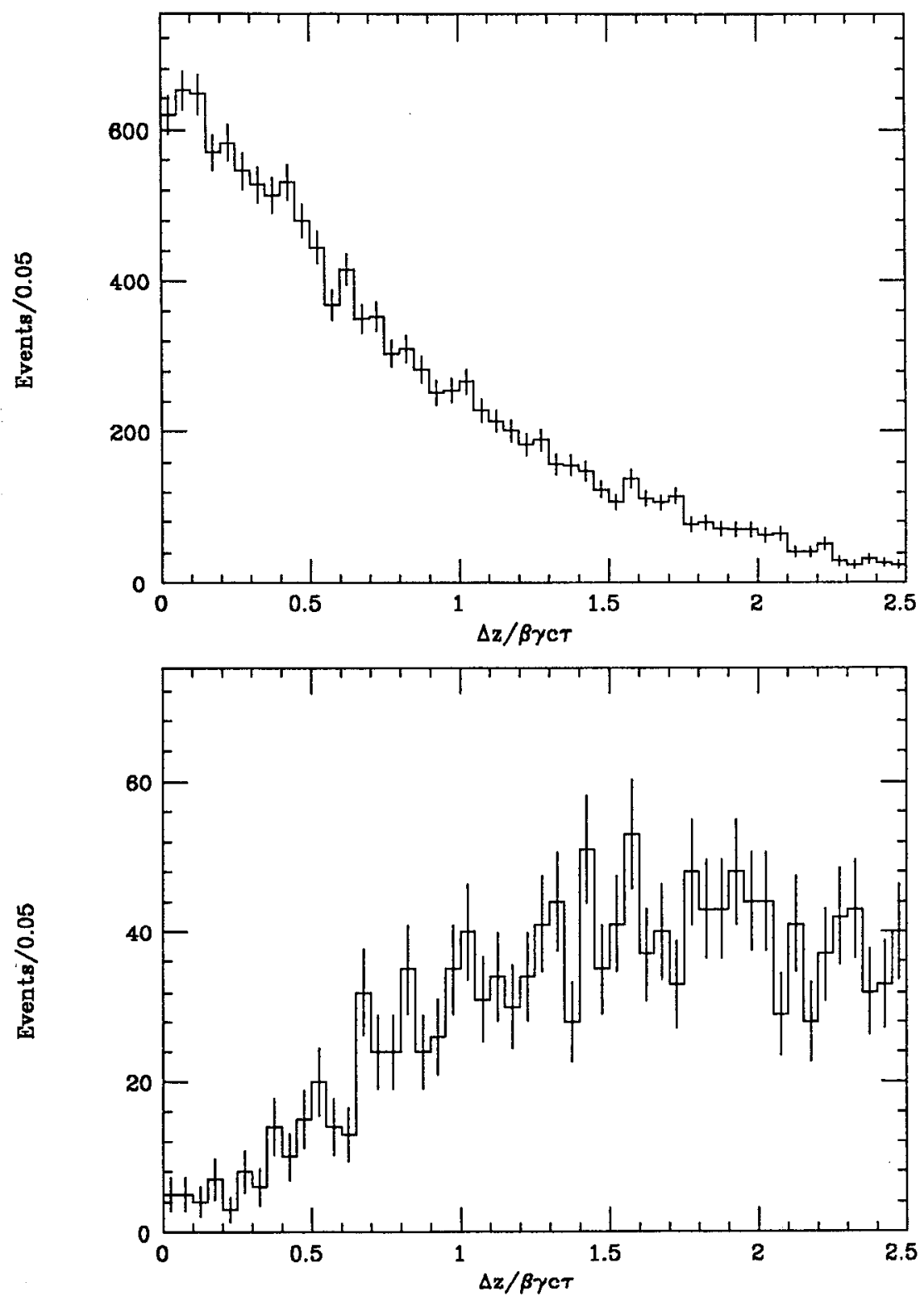

Examples of these event distributions are shown in Figures 6.1 through 6.6. Figures $6.1 \mathrm{a}$ and $6.1 \mathrm{~b}$ show the unsmeared $\Delta t / \tau$ distribution for opposite-sign and like-sign $(C=$ -1) $B_{s} \bar{B}_{s}$ plus $B_{s}^{*} \bar{B}_{s}^{*}$ events when $x_{s}=10$. The luminosity is equal to $30 \mathrm{fb}^{-1}$. Figures $6.2 \mathrm{a}$ and $6.2 \mathrm{~b}$ show the same data after smearing with $\sigma=0.25\langle\Delta z\rangle$. Figures $6.3,6.4$, and 6.5 
show the similar (smeared) distributions for the $(C=+1)$ $B_{s} \bar{B}_{s}^{*}$, and the $B_{d} \bar{B}_{d}$ events. Finally, $6.6 \mathrm{a}$ and $6.6 \mathrm{~b}$ show the sum of all the distributions. The various types of $B_{u} \bar{B}_{u}$ events are included in $6.6 \mathrm{a} ; 6.6 \mathrm{~b}$ is the histogram used for the fits.
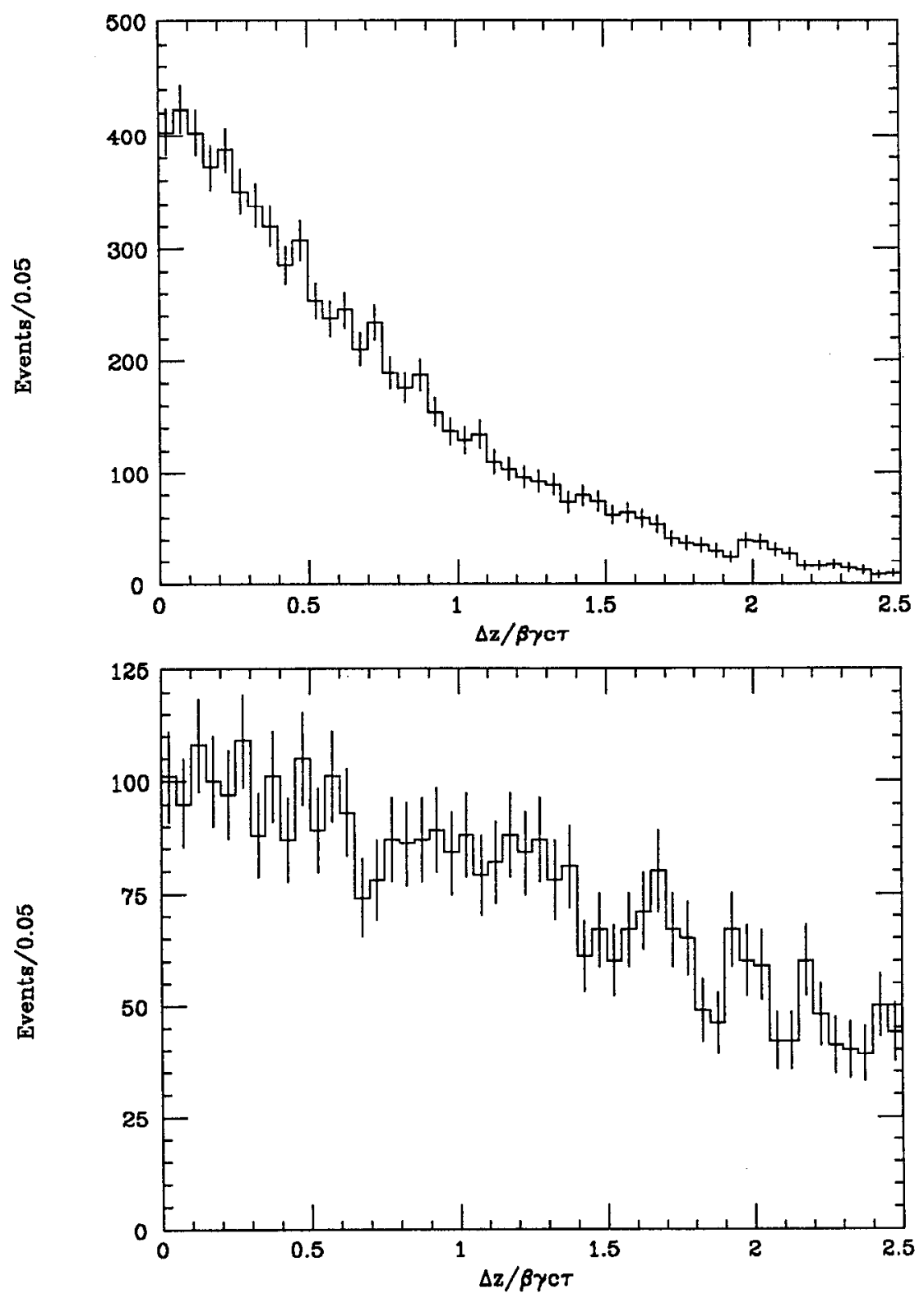

Figure 6.5 a\&b The (smeared) decay path length distribution for $C=+1 \quad B_{d}$ meson pairs; the upper plot is for opposite-sign lepton events, the lower for like-sign lepton events. This represents $30 \mathrm{fb}^{-1}$ of data, with $x_{d}=0.70$ and $\sigma=0.25\langle\Delta z\rangle$.

\section{Extracting $x_{s}$}

The Fitting Function

As can be seen from Figure 6.6, the like-sign event distri- 
Figure 6.6 a\&b The sum of all the distributions from Figures 6.2-6.5, plus the $B_{u}$ contribution to the oppositesign lepton events in the upper plot. The lower plot (6.6b) (for like-sign lepton events) is the histogram which was fit. This represents $30 \mathrm{fb}^{-1}$ of data, with $x_{s}=10, x_{d}=0.70$ and $\sigma=$ $0.25\langle\Delta z\rangle$. bution carries more distinct information than the oppositesign events. Thus this analysis used only the like-sign histogram. Preliminary fits to the opposite-sign histogram gave consistently worse results than those from the like-sign histogram. A simultaneous fit to both histograms may provide additional discrimination, but these have not been tried. In every case, we fit all 50 bins spanning the range $0.0 \leq$ $\Delta z /\langle\Delta z\rangle<2.5$. This range contains $90 \%$ of all events.
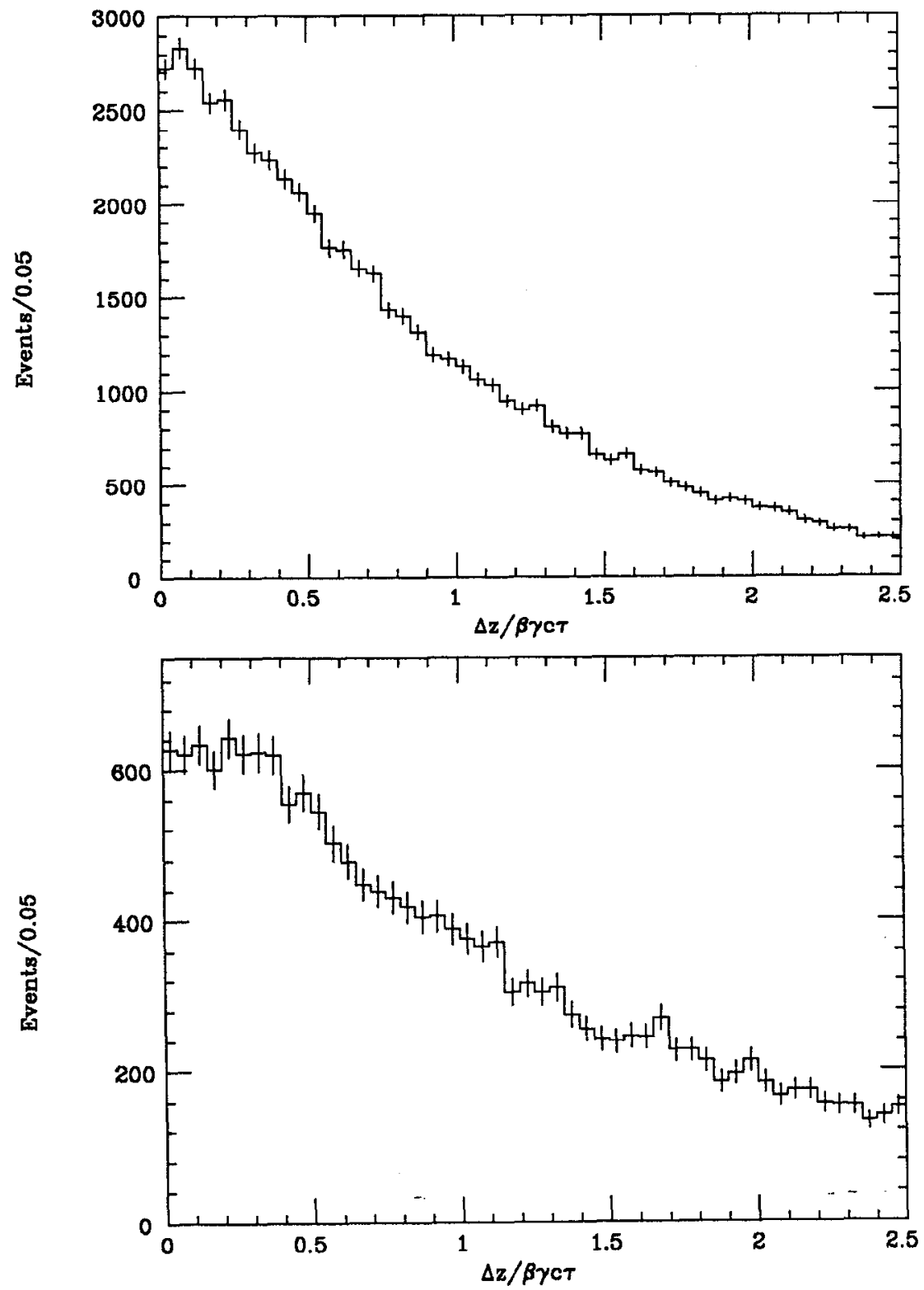
We have treated each of the four components contributing to the like-sign event distribution separately. The $B_{d}$ $C=-1$ and $C=+1$ slowly oscillating components have each been calculated numerically by convoluting a Gaussian with the appropriate time distribution formula. This calculation assumes that $x_{d}$ is known, so that the resulting distributions depend only on the resolution. The convolution was done for each of the seven resolutions. The small $C=+1 B_{s}$ component was treated as purely exponential; this is a good approximation after the true distribution has been smeared. Finally the signal component $\left(C=-1 B_{s}\right)$ was calculated bin by bin by doing a numerical integration twice per bin, splitting each into a lower and an upper half.

There were a total of seven parameters in the fitting function:

(1) $L$ integrated luminosity $\left(\mathrm{fb}^{-1}\right)$

(2) $\sigma \quad$ vertex rcsolution

(3) $N_{d-} \quad B_{d} C=-1$ cross section (fb)

(4) $N_{d+} \quad B_{d} C=+1$ cross section (fb)

(5) $N_{s-} \quad B_{s} C=-1$ cross section (fb)

(6) $N_{s+} B_{s} C=+1$ cross section (fb)

(7) $x_{s} \quad B_{s}$ mixing paramcter.

Only $x_{s}$ was allowed to float. The total luminosity, vertex resolution and total $\bar{B}$ cross section will be well measured; the relative fractions of $B_{u}, B_{d}$ and $B_{s}$, and of the $C=+1$ and $C=-1$ components may be more difficult to determine. In addition, the value of $x_{d}$ was fixed at 0.70 , the value used to generate the events, which is roughly the average of the ARGUS $^{1}$ and CLEO $^{2}$ results.

\section{Procedure}

After a resolution ratio $(\sigma /\langle\Delta z\rangle)$ and an integrated luminosity were selected, a value for $x_{s}$ was chosen. The appropriate histogram of $\Delta z$ for like-sign dilepton events was generated. The same random number seed was used each time events were generated. After examining the histogram, a rough estimate of $x_{s}$ was made and used as input to the fitter with large errors $( \pm 5)$. The fitter then found the best value of $x_{s}$ by first executing the "CHOOSE" procedure (in MINUIT), which does a quick search, randomly varying the input param-
A seven-parameter fit was employed; only $x$, was allowed to float.
MINUIT varies the initial value of $x$, over a wide range, so the analysis is not sensitive to the input value. 
Figure 6.7 The same histogram as in Figure 6.6b, with the fit overlaid. The fit value of $x_{s}=9.53_{-0.27}^{+0.34}$, the input $x_{s}=10$.

If the fit value of $x_{3}$ was correct to within $10 \%$, and the errors were finite, the fit was considered successful. eter and searching for the lowest minimum. This procedure reduces the importance of the input value, thereby eliminating a possible source of bias. Using this starting value, it performed a full minimization procedure, followed by an error analysis in which the true one standard deviation errors are determined by finding the lower and higher values of $x_{s}$ which increase the $\chi^{2}$ by 1 . As an example, Figure 6.7 shows the same data as in $6.6 \mathrm{~b}$, with a fit overlaid.

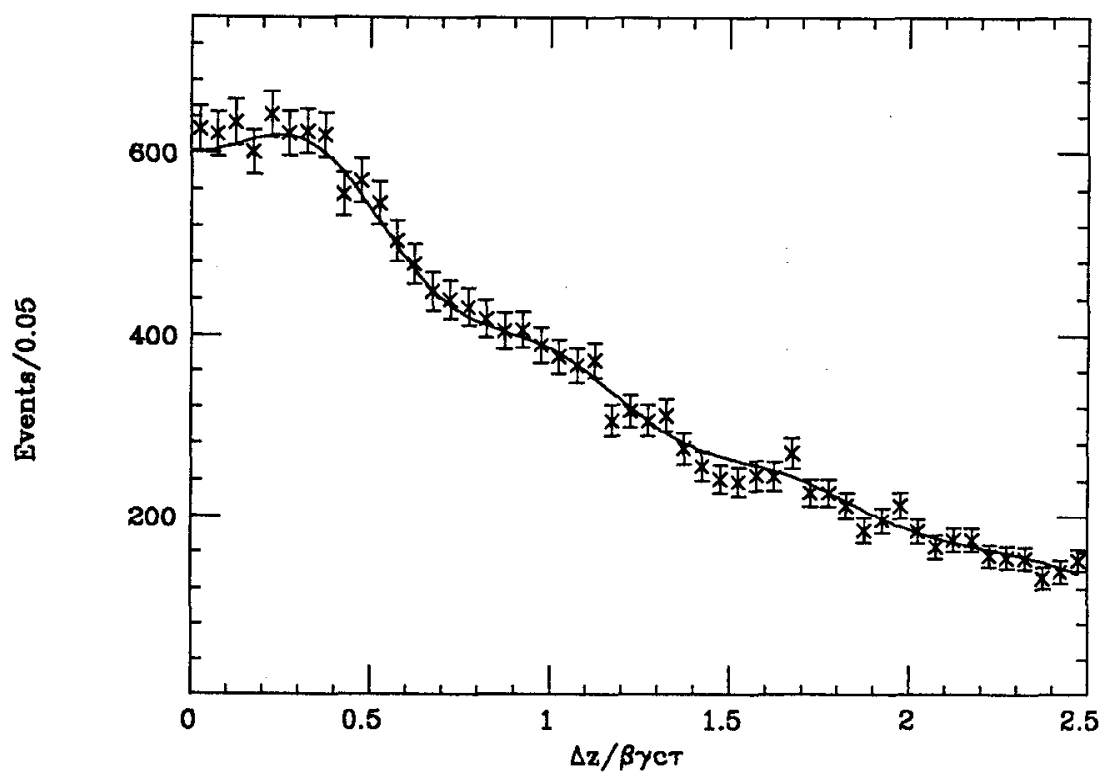

If the fit value of $x_{s}$ was within $10 \%$ of the input value, and if the error bars were finite, this was considered a successful fit (on a few occasions the central fit value was within $10 \%$ of the correct value, but the upper error bar was unbounded; such a case would be useful for setting a lower limit on $x_{s}$, but was not considered successful here). If the fit was successful, $x_{s}$ was increased by 1 and the procedure repeated until a value of $x_{3}$ which failed was found. If the initial value failed, $x_{3}$ was decreased until a successful value was found. $x_{\max }$ was thus determined to be the largest value of $x_{s}$ with a successful fit, plus 0.5 .

In order to estimate the errors on $x_{\max }$, we redetermined $x_{\text {max }}$ for a few points, starting with a different random-number seed. This was repeated up to 10 times to make 10 measurements of $x_{\max }$ at a single point. The errors determined this way are shown as one standard deviation error bars in 
Figures 6.8 and 6.9. This systematic error $(\sim 1-2)$ dominates over the $1 / \sqrt{12}$ error which comes from taking integer steps to determine $x_{\max }$.

\section{Conclusions}

Table 6.2 presents the results for all the cases of the study; they are also presented in Figures 6.8 and 6.9. Notc that thc value of $x_{\max }$ is always quoted as a half-integer, since we made integer steps.

Table $6.2 x_{\max }$ Values

\begin{tabular}{|c|c|c|c|c|c|c|c|}
\hline & \multicolumn{6}{|c|}{ Vertex Resolution $(\sigma /\langle\Delta z\rangle)$} \\
Lumin. & 0.667 & 0.444 & 0.333 & 0.250 & 0.222 & 0.143 & 0.125 \\
\hline $3 \mathrm{fb}^{-1}$ & 3.5 & 6.5 & 6.5 & 9.5 & 11.5 & 15.5 & 19.5 \\
\hline $30 \mathrm{fb}^{-1}$ & 4.5 & 8.5 & 9.5 & 11.5 & 17.5 & 20.5 & 22.5 \\
\hline
\end{tabular}

The figures also show a least-squared fit to a straight line; the functions and their $\chi^{2}$, for 5 degrees of freedom, are as follows:

$$
\begin{array}{lll}
\mathcal{L}=3 \mathrm{fb}^{-1}: & x_{\max }=0.06+2.29\langle\Delta z\rangle / \sigma & \chi^{2}=2.8 \\
\mathcal{L}=30 \mathrm{fb}^{-1}: & x_{\max }=0.08+3.00\langle\Delta z\rangle / \sigma & \chi^{2}=3.3
\end{array}
$$

It is clear that $x_{m a x}$ is proportional to $\langle\Delta z\rangle / \sigma$. The more interesting result is that increasing the luminosity increases the dependence of our $x_{\max }$ capability on $\langle\Delta z\rangle / \sigma$, but does not alter the constant term. This implies that in the region of $\langle\Delta z\rangle / \sigma \sim 3$, which corresponds to the capabilities of our detector with 9 on $3 \mathrm{GeV}$, we will gain only slowly with luminosity. On the other hand, if $x_{s}$ is larger than $x_{\max }$ for our data, we will, at the least, be able to set a lower limit on $x_{s}$ of roughly $x_{\max }$. This will probably be the best limit on $x_{s}$, and is of significant theoretical interest.
Increasing the integrated luminosity increases the slope of the relation between $x_{\max }$ and $\langle\Delta z\rangle / \sigma$ but does not increase the intercept. 
Figure 6.8 $x_{\max }$ as a function of $\langle\Delta z\rangle / \sigma$ for $3 \mathrm{fb}^{-1}$ integrated luminosity.

Figure 6.9 Same as Figure 6.8 , but for $30 \mathrm{fb}^{-1}$ integrated luminosity.

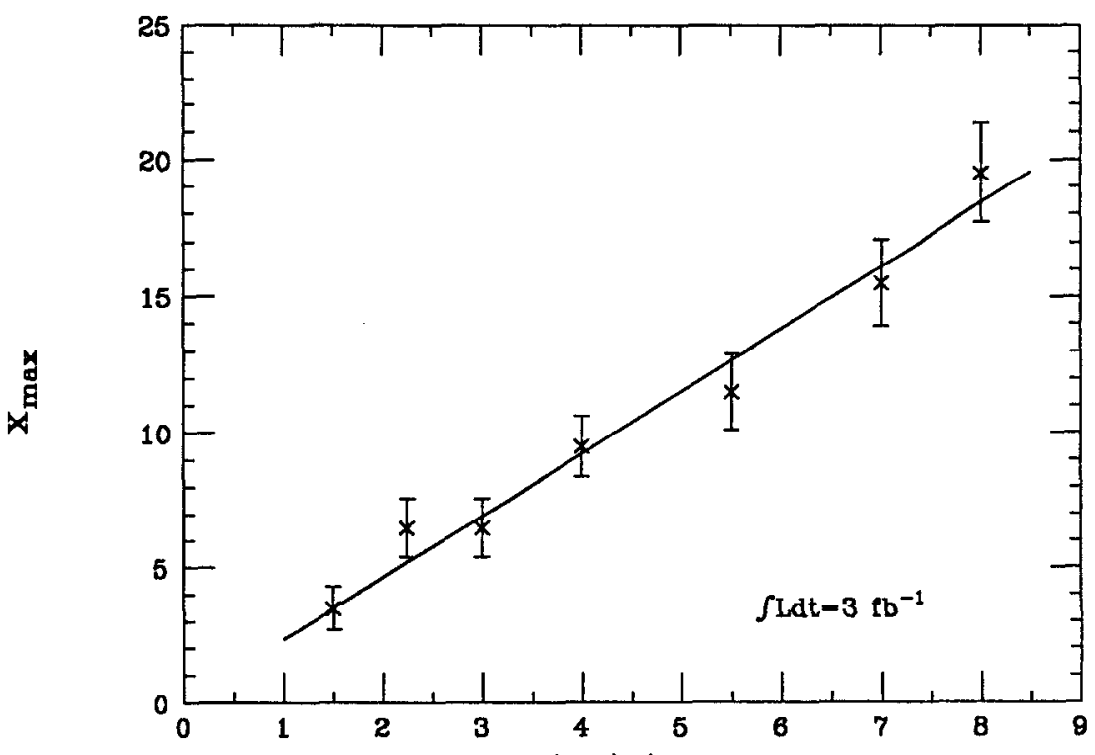

$\langle\Delta z\rangle / \sigma$

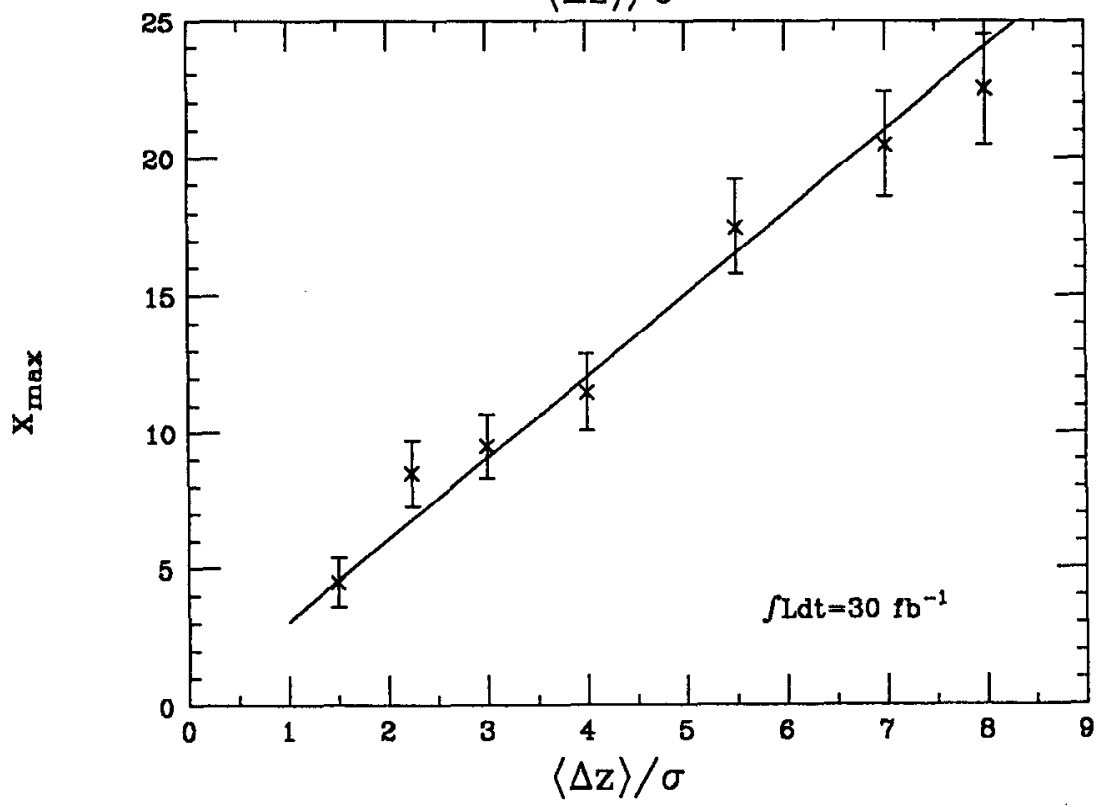




\section{3. $b \rightarrow c$ Hadronic Decays - Understanding the} WEAK DECAY MECHANISM

Introduction

The study of the hadronic weak decays of heavy mesons has, over the last decade, been advanced considerably by experiments in both the $c$ and $b$ quark sector. There is an interesting opposition, however, of experimental and theoretical progress in the two areas. The clearest theoretical predictions are made in the $b$ quark sector, where the experimental measurements are crude. The experimental situation in the charm sector is very much better: there are high precision measurements of exclusive branching ratios and meson and baryon lifetimes. The clear interpretation of these results is, however, complicated by the fact that the energy release in charm decay places the final state hadron relativc momenta in the middle of the resonance region; moreover, non-perturbative effects are relatively large. In the $b$ quark sector, both of these effects are substantially less important, so that more definite predictions can be made. The problem is that the data on hadronic $B$ meson decay are sparse. The total of one million $B$ mesons thus far produced at the $\Upsilon(4 S)$ by CLEO and ARGUS has yielded only a few hundred reconstructed events. There is also no measurement of the individual lifetimes of charged and neutral $B$ mesons. Thus, our understanding of the non-leptonic decays of the $b$ quark remains at a relatively crude level.

A high-luminosity asymmetric $B$ factory promises to produce a much larger sample of $B$ mesons, which together with the reduction in combinatoric background made possible by the reconstruction of individual $B$ meson decay vertices and the improved photon detection and particle identification capability of a new detector, could lead to an increase in the number of reconstructed hadronic $B$ decays by as much as a factor of $10^{3}$. This would bring the experimental precision to a level comparable to that which currently exists in the charm sector. We would thus achieve a detailed understanding of the hadronic current-current interaction in the heaviest experimentally accessible quark system.
Theoretical predictions in the $b$ quark sector are more definite than those in the $c$ quark sector.
With the statistics which could be obtained at an asymmetric $B$ factory, we can achieve an understanding of the hadronic current-current interaction. 
The Model of Bauer, Stech and Wirbel

Bauer, Stech and Wirbel have produced a model ${ }^{8}$ which, assuming factorization, evaluates the effective Lagrangian using quark model wave functions for the hadronic states. The use of quark-model wave functions means that, perforce, this picture deals only with two-body and quasi-two-body final states. This model has been successful in describing the main features of exclusive hadronic charm decay, although, since it incorporates neither non-factorizable amplitudes such as annihilation processes, nor final state interactions, it cannot provide a complete description. Since these contributions are almost certainly much less significant in the $b$ quark sector, the BSW model should come much closer to a complete description of non-leptonic $B$ meson decays. The phemonemological BSW model finds its justification in the $1 / N_{c}$ expansion, as well as in QCD sum rule calculations, ${ }^{10}$ which demonstrate that those terms neglected in the model are of non-leading order in $1 / N_{c}$ and that these terms tend to cancel.

For a brief discussion of this approach, we begin with the $\Delta B=1$ non-leptonic effective Lagrangian (neglecting penguin amplitudes):

$$
\begin{array}{r}
L_{e f f}(\Delta B=1)= \\
\frac{G_{F}}{\sqrt{2}}\left\{V_{c b} V_{u d}^{*}\left[c_{1}(\mu)(\bar{c} b)_{L}(\bar{d} u)_{L}+c_{2}(\mu)(\bar{d} b)_{L}(\bar{c} u)_{L}\right]+\right. \\
V_{c b} V_{u s}^{*}\left[c_{1}(\mu)(\bar{c} b)_{L}(\bar{s} u)_{L}+c_{2}(\mu)(\bar{s} b)_{L}(\bar{c} u)_{L}\right]+ \\
V_{c b} V_{c s}^{*}\left[c_{1}(\mu)(\bar{c} b)_{L}(\bar{s} c)_{L}+c_{2}(\mu)(\bar{s} b)_{L}(\bar{c} c)_{L}\right]+ \\
V_{c b} V_{c d}^{*}\left[c_{1}(\mu)(\bar{c} b)_{L}(\bar{d} c)_{L}+c_{2}(\mu)(\bar{d} b)_{L}(\bar{c} c)_{L}\right]+ \\
V_{u b} V_{u d}^{*}\left[c_{1}(\mu)(\bar{u} b)_{L}(\bar{d} u)_{L}+c_{2}(\mu)(\bar{d} b)_{L}(\bar{u} u)_{L}\right]+ \\
V_{u b} V_{u s}^{*}\left[c_{1}(\mu)(\bar{u} b)_{L}(\bar{s} u)_{L}+c_{2}(\mu)(\bar{s} u)_{L}(\bar{u} u)_{L}\right]+ \\
V_{u b} V_{c s}^{*}\left[c_{1}(\mu)(\bar{u} b)_{L}(\bar{s} c)_{L}+c_{2}(\mu)(\bar{s} b)_{L}(\bar{u} c)_{L}\right]+ \\
\left.V_{u b} V_{c d}^{*}\left[c_{1}(\mu)(\bar{u} b)_{L}(\bar{d} c)_{L}+c_{2}(\mu)(\bar{d} b)_{L}(\bar{u} c)_{L}\right]\right\} .
\end{array}
$$


where the QCD renormalization group coefficients

$$
c_{1}=\frac{c_{+}+c_{-}}{2} \text { and } c_{2}=\frac{c_{+}-c_{-}}{2}
$$

are evaluated at the appropriate scale $\mu \sim 5 \mathrm{GeV}$. The notation $(\bar{s} c)_{L}$ stands for the $V-A$ color-summed operator $(\bar{s} c)_{L}=\sum_{i=1}^{3} \bar{s}_{i} \gamma^{\mu}\left(1-\gamma_{5}\right) c_{i}$. Note that the QCD correction induces an "effective neutral current" coupling; the pure charged current form is recovered in the limit $c_{1} \rightarrow 1, c_{2} \rightarrow 0$, as $\alpha_{s} \rightarrow 0$.

The BSW method consists of evaluating this Lagrangian in a specific quark model. The various terms are interpreted as effective hadron, rather than quark, field operators. Thus the first term would be rewritten as

$$
a_{1}(\mu)(\bar{c} b)_{H}(\bar{s} u)_{H}
$$

The hadron field operators are to be explicitly evaluated using factorization and quark model wave functions.

There is a relation between the coefficients $c_{i}$ and $a_{i}$ :

$$
a_{1}=c_{1}+\left.\xi c_{2}\right|_{\mu \simeq m_{c}, m_{b}}, a_{2}=c_{2}+\left.\xi c_{1}\right|_{\mu \simeq m_{c}, m_{b}}
$$

The color factor $\xi=1 / N_{c}$ arises from the color mismatch involved in forming color singlets from the Fierz-transformed terms in the Lagrangian. Its actual value may differ from $1 / 3$ due to non-perturbative effects, particularly in the charm sector. At the scale $\mu=m_{b}$ we expect

$$
c_{1} \simeq 1.1, c_{2} \simeq-0.24
$$

Thus, the most basic task of an experimental investigation of the mechanism of $B$ meson hadronic decay is to establish the values of $a_{1}, a_{2}$ and $\xi$, and to compare these with values derived from QCD-based models, such as the $1 / N_{c}$ expansion.
The terms in the effective Lagrangian are interpreted as hadronic field operators.

The BSW coefficients are directly extracted from measurements of $B$ hadronic decay branching ratios. 
Current $B$ meson branching ratio measurements are based on, at best, tens of events per exclusive channel.

The reconstruction efficiency for $B$ meson decay channels proceeding through a $D$ or $D^{*}$ meson is necessarily small.
Particular exclusive decay modes may have decay widths proportional to $a_{1}^{2}, a_{2}^{2}$ or $\left(a_{1}+K a_{2}\right)^{2}$. For example:

$$
\begin{aligned}
B\left(\bar{B}^{o} \rightarrow D^{+} \pi^{-}\right) & \sim 0.48 a_{1}^{2} \\
B\left(B^{-} \rightarrow K^{-} J / \psi\right) & \sim 1.01 a_{2}^{2} \\
B\left(B^{-} \rightarrow D^{o} \pi^{-}\right) & \sim 0.48\left(a_{1}+0.75 a_{2}\right)^{2},
\end{aligned}
$$

where factors proportional to K-M angles and phase space have been suppressed.

Thus, measurements of individual branching fractions to sufficient precision can allow the extraction of the $a_{1}$ and $a_{2}$ coefficients. Comparison with the calculated values of $c_{1}$ and $c_{2}$ allows extraction of the value of $\xi$. In the charm sector, the value of $\xi$ so derived is $0.10 \pm 0.15$, which is consistent both with the naive value of $1 / 3$ and with zero, which would indicate that color-suppressed amplitudes are absent. While the complexity of the charm decay process makes it difficult to further refine this analysis, in the $b$ sector this ansatz provides a far more complete description, and thus improved data on $B$ meson decay branching fractions should allow explicit confrontation with theory. Current measurements, based on a handful of events into any particular exclusive hadronic channel, are not capable of shedding light on the problem. The order-of-magnitude increase in statistics we can expect from $\Upsilon(4 S)$ experiments in the next several years will also not provide sufficient precision to clearly establish the value of $\xi$ in the $B$ mson system.

\section{$B$ Meson Reconstruction Efficiency}

The experimental problem facing us is the small branching ratios of $B$ mesons to particular exclusive channels, and the relatively low efficiency of reconstruction of many major channels. A low efficiency for reconstruction of $B$ meson decays which proceed through $D^{*}$ or $D$ channels (the vast majority) is inescapable. If we restrict ourselves to the reconstruction of $D^{+}$mesons through the modes $K_{S}^{o} \pi^{+}, K_{S}^{o} \pi^{+} \pi^{o}$, $K_{S}^{\circ} \pi^{+} \pi^{+} \pi^{-}, K^{-} \pi^{+} \pi^{+}$and $K^{-} \pi^{+} \pi^{+} \pi^{\circ}$, we have a typical $D^{+}$reconstruction efficiency of $10 \%$. For $D^{0}$ decays, reconstructed through the modes $K^{-} \pi^{+}, K^{-} \pi^{+} \pi^{0}, K^{-} \pi^{+} \pi^{+} \pi^{-}$, $K_{S}^{o} \pi^{+} \pi^{+} \pi^{-}$and $K_{S}^{o} \pi^{o}$, the efficiency is higher, at $14 \%$. For 
$D_{s}^{+}$decays, reconstructed through the modes $\phi \pi^{+}, \phi \pi^{+} \pi^{o}$, $K_{S}^{o} K^{+}$and $K^{* o} K^{+}$, the efficiency is $1.1 \%$. For $D^{*+}$ and $D^{* o}$, the overall reconstruction efficiency, including the $D$ meson reconstruction efficiency, is $10 \% .^{\# 1}$ The excellent resolution in $M_{D^{*}}-M_{D}$ produces very clean reconstructed $B$ signals. We will quote numbers of reconstructed $B$ meson events under the very conservative assumption that it is possible to reconstruct charmed mesons with an acceptable signal-to-noise ratio in only the simplest all-charged-particle decay mode. This reduces the reconstruction efficiency to $2.7 \%$ for $D^{0}$ (via $K^{-} \pi^{+}$), to $5.4 \%$ for $D^{+}\left(\right.$via $K^{-} \pi^{+} \pi^{+}$) and to $0.7 \%$ for $D_{s}^{+}$ (via $\phi \pi^{+}$). It also reduces the $D^{*+}$ efficiency to $3.1 \%$ and the $D^{* o}$ efficiency to $2.0 \%$. This approach produces very clean $B$ signals, suitable for those experiments which depend on recoil tagging.

Table 6.3 summarizes the number of $B$ mesons which can be completely reconstructed in integrated data samples corresponding to our standard $30 \mathrm{fb}^{-1}$ and $300 \mathrm{fb}^{-1}$ luminosities at the $\Upsilon(4 \mathrm{~S})$, under the assumptions stated above. The $B$ meson branching ratios used are those of CLEO, reported ${ }^{11}$ at the recent Lepton/Photon Conference, for charged particle modes, and those of ARGUS reported ${ }^{12}$ at the 1989 Heavy Quark Symposium, for modes involving $\pi^{o}$ 's. For comparison, it is worth noting that the largest extant $\Upsilon(4 S)$ sample (the CLEO 1987 sample) has yielded about 15 events in the efficiently reconstructed $\bar{B}^{o} \rightarrow D^{*+} \pi^{-} \pi^{-} \pi^{+}$mode.

Thus, even with the initial sample, a very large number of $B$ mesons will be reconstructed, even if we restrict ourselves to the use of all-charged modes only. The excellent vertex reconstruction and $\pi^{\circ}$ detection capability of a new detector will certainly allow the use of other modes in reconstructing $B$ mesons.

\#1 We use the Mark III branching ratios for $D^{\circ}, D^{+}$and $D^{*}$ decays and assume that all $D_{3}^{+}$decays used have a $3 \%$ branching ratio. 
Table 6.3 Number of reconstructed $B$ meson hadronic decays

\begin{tabular}{|c|c|c|c|c|}
\hline Decay Mode & \multicolumn{2}{|c|}{$30 \mathrm{fb}^{-1}$ Sample } & \multicolumn{2}{|c|}{$300 \mathrm{fb}^{-1}$ Sample } \\
& Normal & Charged $D$ & Normal & Charged $D$ \\
& $D$ & Mode Only & $D$ & Mode Only \\
\hline $\bar{B}^{\circ} \rightarrow D^{+} \pi^{-}$ & 8300 & 4400 & 83000 & 44000 \\
$\vec{B}^{o} \rightarrow D^{*+} \pi^{-}$ & 10000 & 3100 & 100000 & 31000 \\
$B^{-} \rightarrow D^{*+} \pi^{-} \pi^{-}$ & 5500 & 1700 & 55000 & 17000 \\
$\bar{B}^{\circ} \rightarrow D^{+} \pi^{-} \pi^{o}$ & 40700 & 22000 & 407000 & 220000 \\
$B^{-} \rightarrow D^{o} \pi^{-} \pi^{o}$ & 54700 & 10800 & 547000 & 10800 \\
$\bar{B}^{o} \rightarrow D^{+} D_{s}^{-}$ & 790 & 270 & 7900 & 2700 \\
$\bar{B}^{o} \rightarrow D^{*+} D_{s}^{-}$ & 1200 & 230 & 12000 & 2300 \\
\hline
\end{tabular}

Samples of these large sizes allow the determination of the BSW coefficients to a statistical accuracy of far better than $1 \%$; at this level, conclusions will be limited by systematic uncertainties involving calculation of reconstruction efficiency, luminosity determination, and the accuracy of charmed meson branching fraction measurements, which should soon be improved by results from $\mathrm{BEPC}$ and the new generation of fixed-target experiments.

A further use of these large, clean samples will be their use as tags, much as been done with $D$ mesons by Mark III. Having a large sample of very clean tagged events allows the reconstruction of exclusive semileptonic decay modes, as well as the search for rare decays such as $B \rightarrow \tau \nu_{\tau}$. Examples of such uses of tagged events will be discussed later in this chapter. 
While the semi-leptonic decays of $B$ mesons cannot be fully reconstructed due to the missing neutrino, they can be partially reconstructed using the fact that $B$ mesons are produced almost at rest in the $\Upsilon(4 S)$ rest frame. Such tags are still useful for many purposes, and since the semi-leptonic decays have much larger branching fractions then the fully reconstructable modes, e.g., $D^{*} \pi$, much larger samples of $B$ mesons can be tagged by this technique.

We consider the following decay modes:

a. $B^{0} \rightarrow D^{*+} l^{-} \nu, D^{*+} \rightarrow D^{0} \pi^{+}$

b. $B^{0} \rightarrow D^{*+} l^{-} \nu, D^{*+} \rightarrow D^{+} \pi^{0}$

c. $B^{-} \rightarrow D^{0} l^{-} \nu$

d. $B^{-} \rightarrow D^{* 0} l^{-} \nu, D^{* 0} \rightarrow D^{0} \pi^{0}$

For the reconstruction of the $D^{0}$ we assume that all decay modes with no more than one $\pi^{0}$ or one $K_{S}$ can be reconstructed. Table 6.4 gives the resulting efficiency for these four channels.

Table 6.4 $B$ Meson partial reconstruction efficiencies

\begin{tabular}{|c|c|c|}
\hline Mode & Sum of branching fractions & Overall efficiency \\
\hline a. & $2.5 \%$ & $0.65 \%$ \\
\hline b. & $2.1 \%$ & $0.25 \%$ \\
\hline c. & $1.7 \%$ & $0.50 \%$ \\
\hline d. & $2.5 \%$ & $0.40 \%$ \\
\hline
\end{tabular}

The calculations asume that $90 \%$ of the charged tracks and $60 \%$ of the $\pi^{0}$ 's can be reconstructed. Lepton indentification is assumed for all reconstructed tracks with momenta grcater than $0.5 \mathrm{GeV}$. These calculations have been checked against the Monte Carlo for the $D$ decay channels $K^{-} \pi^{+}$, $K^{-} \pi^{+} \pi^{-} \pi^{+}, K^{-} \pi^{+} \pi^{-}$and $K^{-} \pi^{+} \pi^{0}$. They agree to better than $20 \%$ in all cases.

We have made preliminary Monte Carlo studies of the backgrounds in these channels. Backgrounds in mode (a) are very small. For (b) and (d), the modes involving a slow $\pi^{0}$, 
Measuring $\left|V_{u b}\right|$ provides important constraints on the Standard Model. there is a strong tendency to pick up the wrong $\pi^{0}$. There is also strong feed down of $D^{*}$ modes into (c). However, when the decay on the other side does not involve slow $\pi^{0}$ 's, they provide a reasonably clean tag.

In a $300 \mathrm{fb}^{-1}$ data sample we can expect $\approx 10^{6}$ tags in each of these channels. This should allow us to study rare decays a the $10^{-5}$ level.

\section{5. $b \rightarrow u$ DECAYS - Determination of $V_{u b}$}

\section{Introduction}

There are several reasons for studying those decays of $B$ mesons which are expected to occur via $b \rightarrow u$ transitions. First, such decays provide the possibility of determining the magnitude of the Kobayashi-Maskawa matrix element $V_{u b}$. This measurement alone is an important goal for any $B$ physics program. In the three-generation Standard Model, all elements in the KM matrix must be non-zero in order to explain CP violation in $\mathrm{K}$ decays. From this one obtains a theoretical lower limit, $\left|V_{u b} / V_{c b}\right| \geq 0.05$, or for $\left|V_{c b}\right| \sim 0.05$, $\left|V_{u b}\right| \geq 2.5 \times 10^{-3}$. An accurate measurement of $\left|V_{u b}\right|$ would provide an important ingredient in constraining the Standard Model via the so-called Unitarity Triangle, as described in Chapter 2.

Second, as discussed in Chapter 5 , the measurement of $\mathrm{CP}$-violation in $B^{0}$ and $\bar{B}^{0}$ decays to $\mathrm{CP}$-eigenstates reached via $b \rightarrow u$ transitions would provide information distinct from that accessible with $b \rightarrow c$ decays. In particular, such a measurement of CP violation would determine $\sin 2 \alpha$, thus providing another constraint on the Unitarity Triangle. Third, one would like to extend the studies of strong interaction effects in weak $B$ decays ( $c$.f. Section 6.3 for $b \rightarrow c$ decays) to the case in which the final state contains no heavy quark.

Three approaches have been considered for experimentally determining $V_{u b}$ at a $B$ factory:

1. Inclusive semileptonic decays

2. Exclusive semileptonic decays

3. Exclusive hadronic decays 
Inclusive Semileptonic Decays

The traditional method has been ${ }^{13}$ has been the study of the endpoint region of the inclusive lepton momentum spectrum. Because of the large mass of the $c$ quark compared to that of the $u$ quark, the highest-momentum leptons should arise only from the quark-level decay $b \rightarrow u l^{-} \bar{\nu}$. At the 1989 Lepton-Photon Symposium, both CLEO and ARGUS presented evidence for such decays at the $2.2 \sigma$ and $3.2 \sigma$ levels, repectively. While not yet statistically compelling, this is the closest we now have to confirmed evidence for $b \rightarrow u$ decays of $B$ mesons. If the effects are real, they imply that $\left|V_{u b} / V_{c b}\right| \sim 0.1$. However, the central values are rather model-dependent: CLEO finds a factor of 2 range for this ratio, depending upon the choice of the model used to relate the experimental yields to $\mathrm{KM}$ matrix elements. Uncertainties in the models also affect the assumed shape of the $b \rightarrow c$ lepton momentum spectrum, which must be subtracted or fit to obtain the $b \rightarrow u$ yields.

We expect that by the time a high-luminosity $B$ factory is built, Method 1 will have reached the limit of systematic and theoretical uncertainties. Moreover, Isgur and his collaborators ${ }^{14,15}$ have called into question the use of free-quark decay models to describe a kinematic region which is in fact dominated by specific single-hadron states. They maintain that the inclusive spectrum near the endpoint should be obtained as an explicit sum of exclusive contributions.

For these reasons, our discussion of the determination of $\left|V_{u b}\right|$ via semileptonic decays will focus on the complementary approach of Method 2, involving exclusive modes. The simplest such modes are $B \rightarrow \pi l^{+} \nu$ and $B \rightarrow \rho l^{+} \nu$. Calculation of their yields should be less theoretically uncertain than in the inclusive semi-leptonic case. However, the calculation does involve transition form factors whose shapes are measurable but whose absolute normalization must come from a model. Hence extracting $\left|V_{u b}\right|$ from such measurements is also not free of theoretical uncertainties. Given adequate statistics, however, there is a great wealth of information which can in principle be extracted from detailed studies of these decays, ${ }^{16}$ as well as from exclusive $b \rightarrow c$ and $c \rightarrow s$ decays,
We will focus on the exclusive semi-leptonic decay modes, on the assumption that the inclusive semi-leptonic measurements will soon reach their theoretical and systematic limits. 
There are large theoretical uncertainties in relating exclusive hadronic branching ratios to $\left|V_{u b}\right|$. allowing one to confront QCD models in considerable detail. It is crucial that there be a significant signal-to-background ratio over the entire Dalitz plot if we are to measure the fourmomentum-tranfer-squared $\left(q^{2}\right)$ dependence of the form factors. This cannot be done without precise vertex information.

Finally, one can explore exclusive hadronic modes such as $B^{\circ} \rightarrow \pi^{+} \pi^{-}, B^{\circ} \rightarrow \rho^{\circ} \rho^{\circ}$ and $B^{\circ} \rightarrow \omega^{\circ} \pi^{\circ}$. These final states can be fully reconstructed with high efficiency, in contrast to the more difficult partial reconstruction necessary in semi-leptonic decays. Theoretical predictions for these modes exist, ${ }^{8}$ but there are large uncertainties in relating the quark level dynamics to the resulting hadronic final state. Until these models have been fully tested in a variety of final states, there will be a significant theoretical error involved in relating $\operatorname{Br}\left(B^{\circ} \rightarrow \pi^{+} \pi^{-}\right)$or $\operatorname{Br}\left(B^{\circ} \rightarrow \rho^{\circ} \rho^{\circ}\right)$ to $\left|V_{u b}\right|$. However these final states are still very important to study, because they could in principle allow one to measure two parameters of the Unitarity Triangle: one side, given by $\left|V_{u b}\right|$, which is related to the branching fraction, and one angle, $\sin 2 \alpha$, which is related to the $\mathrm{CP}$-violating asymmetry.

A high-luminosity, asymmetric $B$ factory would provide sufficient data to explore in some depth the exclusive $b \rightarrow u$ decays of $B$ mesons, which have not yet even been convincingly established. Furthermore, as the analyses presented below will demonstrate, at an asymmetric machine there are vertex constraints available which significantly improve the signal to background ratio and enhance the discovery potential for these rare decays.

Exclusive Semileptonic $b \rightarrow u$ Decays

To lowest order in the weak interaction, a process $B \rightarrow$ $\mathrm{Ml}^{+} \nu$ (where $M$ is a light-quark meson) is mediated by a single $W$ boson of four-momentum

$$
q=p_{l}+p_{\nu}=p_{B}-p_{M}
$$

The invariant $q^{2}$, which is equal to $m_{l \nu}^{2}$, can be in the range

$$
m_{l}^{2} \leq q^{2} \leq\left(m_{B}-m_{M}\right)^{2}
$$

where the upper limit corresponds to $M$ at rest in the $B$ rest frame. The amplitude is proportional to $V_{u b}$ times the 
scalar product of a known leptonic current with a hadronic weak current. The hadronic current is in turn given by a sum of terms, each the product of a known kinematic factor times a form factor which depends only on $q^{2}$. In the case of light leptons, a pseudoscalar $M$ (i.e., $\pi$ ) involves a single form factor, while a vector $M$ (i.e., $\rho$ ) involves three form factors. (If $m_{l}$ cannot be ignored, there is one additional form factor in each case.) Thus the differential decay rate is

$$
d \Gamma\left(B \rightarrow \pi l^{+} \nu\right)=\left|V_{u b}\right|^{2} \cdot\left|f_{+}\left(q^{2}\right)\right|^{2} \cdot \text { known factors }
$$

The corresponding formula for $B \rightarrow \rho l^{+} \nu$ is a sum of terms, each involving one of the (correlated) $W$ and $\rho$ density matrix elements in a helicity basis. Each helicity amplitude is a linear combination of the form factors, leading to complicated $q^{2}$ dependent interference effects. The complete formulas, as well as detailed derivations, are given in Ref. 16.

Thus if we know the form factors, $\left|V_{u b}\right|$ can be extracted from a measurement of either $B \rightarrow \pi l^{+} \nu$ or $B \rightarrow \rho l^{+} \nu$. A common assumption is that each form factor is dominated by a single pole, at the mass of the closest spin-1 meson with the right parity and flavor-composition. For $B \rightarrow \pi l^{+} \nu$ only the Vector (as opposed to Axial Vector) current contributes, so that pole would be at the $B^{*}$. That is, the pole is at $q^{2} \approx$ $28.4 \mathrm{GeV}^{2}$, very close to the maximum physical $q^{2}, 26.4 \mathrm{GeV}^{2}$. Hence the predicted total rate is very sensitive to the assumed shape of $f_{+}\left(q^{2}\right)$. For $B \rightarrow \rho l^{+} \nu$, the individual form factors are less steeply varying, but this is compensated for by the interference effects, so the sensitivity is still great. ${ }^{16}$ Moreover, we are dealing with transition form factors, so that even the vector form factors are not constrained at some normalizing point. As a result, various QCD models ${ }^{15,18,19,20}$ give total rate predictions differing by a factor of four for the same $\left|V_{u b}\right|$. If we take $\left|V_{u b}\right|=0.005$, then typical predicted branching ratios, for a particular lepton, range from $\sim 0.6$ to $2.2 \times 10^{-4}$ for $B \rightarrow \pi l^{+} \nu$ and from $\sim 2.5$ to $10 \times 10^{-4}$ for $B \rightarrow \rho l^{+} \nu$ : In fact, comparison to experiment for $D$ decays, for which the models tend to differ less, shows that none of them is entirely correct. ${ }^{16}$
To extract $\left|V_{u b}\right|$, one needs to know the form factors (one for $B \rightarrow \pi l^{+} \nu$, three for $\left.B \rightarrow \rho l^{+} \nu\right)$. 
The $q^{2}$ shapes and the ratios of the form factors can in principle - and should - be measured. Obtaining enough statistics to do this will require a $B$ factory.

We have carried out a Monte Carlo study of $B \rightarrow \pi l^{+} \nu$
In order to extract a believable value of $\left|V_{u b}\right|$ from measurements of semileptonic $B$ decays, it is thus necessary to actually measure the form factors. Fortunately, given adequate statistics and an understanding of experimental systematics, this is in principle achievable: for $B \rightarrow \pi l^{+} \nu$ one needs to measure the $q^{2}$-dependence of the decay rate; while for $B \rightarrow \rho l^{+} \nu$ one can determine the shapes and ratios of the form factors by measuring the joint angular decay distributions ${ }^{16}$ of the $W$ and $\rho$ as a function of $q^{2}$. The overall normalizations must still be taken from theory, but these measurements, along with similar ones for corresponding $b \rightarrow c$, $c \rightarrow s$ and $c \rightarrow d$ decays, should heavily constrain acceptable models.

We have carried out a Monte Carlo study of the process $e^{+}+e^{-} \rightarrow \Upsilon(4 \mathrm{~S}) \rightarrow B^{0} \overline{B^{0}}$ with $B^{0} \rightarrow \pi^{-} l^{+} \nu$ (or complex conjugate, where $l$ is either $e$ or $\mu$ ), for the generic detector of Chapter 3. Beam energies were 9.00 on $3.11 \mathrm{GeV}$. The "other" B is treated by Lund quark-decay algorithms. For purposes of this analysis, we assumed perfect identification of detected charged particles; we do not expect this to affect the conclusions significantly. The form factor $f_{+}$was taken to be a simple pole at $5.329 \mathrm{GeV}$. Figure 6.10 shows the generated $q^{2}$ distribution, both for phase space and with the full decay amplitude. (Because $B \rightarrow W+\pi$ is a $p$-wave decay, and large $q^{2}$ is correlated with low pion momentum in the $B$ rest frame, the angular momentum barrier largely counters the large form factor in this region.)

The analysis procedure, both for the signal of interest and for background studies to be described, was as follows:

(1) In each event, find all candidate $\pi^{\mp} l^{ \pm}$pairs, with each particle having $p_{T} \geq 0.5 \mathrm{GeV}$, and $m(l \pi) \geq 1.0 \mathrm{GeV}$.

(2) For each pair, let all the remaining detected particles form a loose 'tag'. Accept only those tags with invariant mass between 4.75 and $5.50 \mathrm{GeV}$. (Events below this range are not adequately constrained; and only background contributes above it.) 

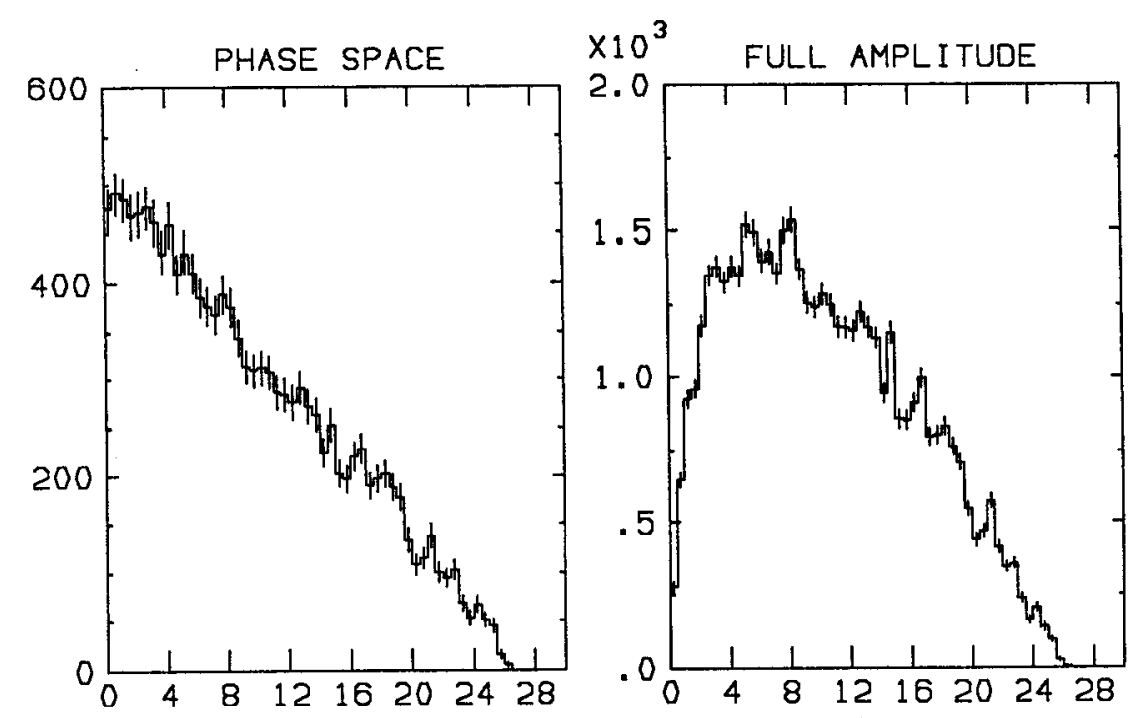

(3) After the tagging cuts, a surviving candidate for the decay of interest is defined to have $\vec{p}_{B}=\vec{p}_{\Upsilon}-\sum \vec{p}_{i}$, where $i$ runs over the particles making up the tagging $B$. The four-vector $p_{B}$ is completed by assigning the true $B^{0}$ mass. We define $p^{*}$ as $\left|\vec{p}_{B}\right|$ in the overall centerof-momentum $(\Upsilon(4 \mathrm{~S})$ rest) frame. The four-vectors $q$ and $p_{\nu}$ are computed from $p_{B}$ and the four-momenta of the candidate $l$ and $\pi$.

Fig. 6.11 shows the distribution at this stage of $p^{*}$ versus the reconstructed neutrino mass-squared for the signal of interest. The sharp peak around the expected values provides two-dimensional discrimination against most sources of background. This may be seen from Fig. 6.12, which shows the same distribution for general $\Upsilon(4 \mathrm{~S}) \rightarrow B \bar{B}$ Monte Carlo events.

(4) Cut on $p^{*}$ and $m_{\nu}{ }^{2}$, accepting only the peak.

(5) Finally, the $\pi^{\mp}$ and $l^{ \pm}$must be consistent with originating from a common vertex. (No cuts have been made on the location of that vertex or on the overall vertex configuration.) A simple cut against all tracks in the
Figure 6.10 Generated $q^{2}$ distributions in $\mathrm{GeV}^{2}$ for $B \rightarrow \pi l^{+} \nu$. The full amplitude includes a simple pole form factor.
A key to separating the $B \rightarrow \pi l^{+} \nu$ signal from background is the two-dimensional constraint in $m_{\nu}^{2}$ vs. $p^{*}$. 
Figure 6.11 Reconstructed $m_{\nu}{ }^{2}$ vs. $p^{*}$ (three-momentum of $B$ in overall c.m. frame) for Monte Carlo $B^{0} \rightarrow \pi^{-} l^{+} \nu$ events.

Figure 6.12 Reconstructed $m_{\nu}{ }^{2}$ vs. $p^{*}$ (three-momentum of the $B$ in overall the c.m. frame) for Monte Carlo $B \bar{B}$ background events.
TAGGED $B-->P I-L-N U$

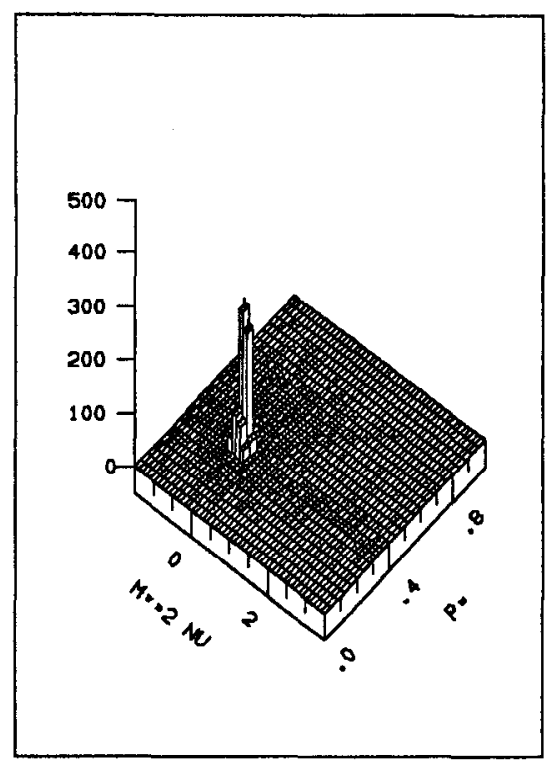

B-BBAR EACKGROUND

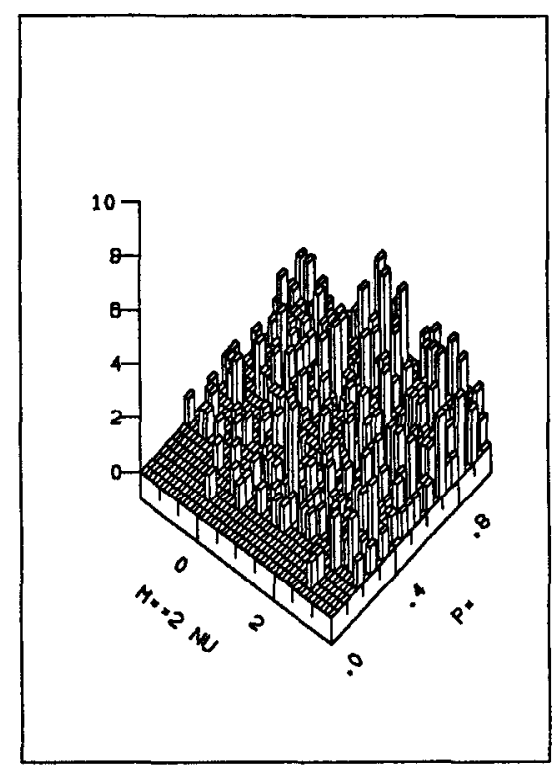

the event arising from a single vertex - as used in the $B^{\circ} \rightarrow \pi^{+} \pi^{-}$analysis described in the succeeding subsection - is ineffective here; the dominant backgrounds are no more consistent with this than is the signal of interest.)

Fig. 6.13 shows the $m_{\nu}{ }^{2}$ spectrum for the signal after all cuts except that on $m_{\nu}{ }^{2}$. 


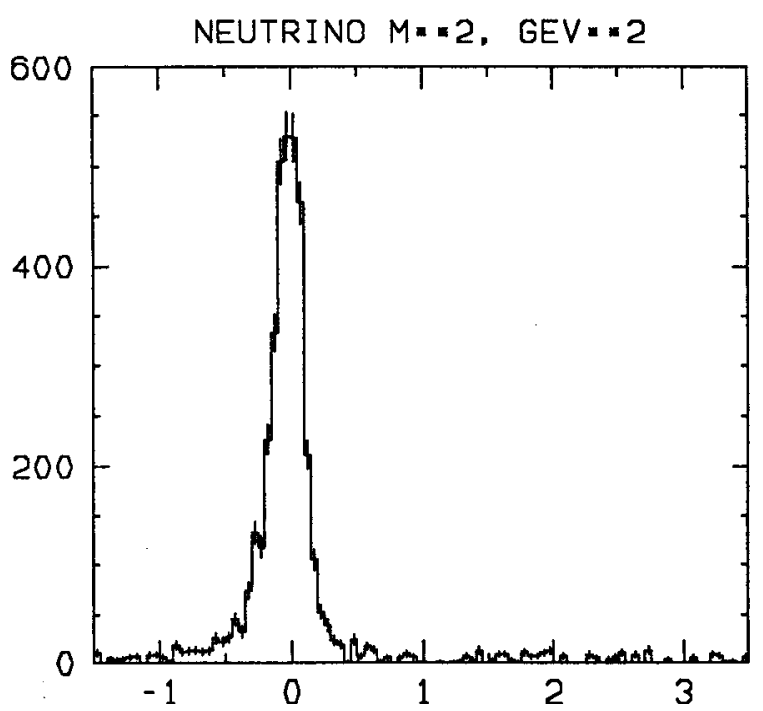

The efficiency to detect the correct $\pi$ and $l$ is 0.85 . The steps outlined above have associated efficiency factors of $\epsilon_{1}=$ $0.83, \epsilon_{2}=0.23$ (tagging efficiency), $\epsilon_{4}=0.44$, and $\epsilon_{5}=0.98$, for an overall efficiency of $\epsilon=0.070$. The accepted area in $p^{*}$ vs. $m_{\nu}{ }^{2}$, and hence the background, can be reduced by a factor of 2 at a cost of reducing $\epsilon$ to 0.059 . To estimate the total yield, we have taken a branching ratio (for each of $l=e, \mu)$ of $1.2 \times 10^{-4}$. Then in the initial sample of $30 \mathrm{fb}^{-1}$ we would detect 440 signal events (with the more stringent requirements). According to the Monte Carlo simulation, the $B \bar{B}$ background would be $\sim 340$ events, and the continuum background, with no special event-shape cuts, $\sim 150$ events. We have also studied two specific sources of background: $B^{0} \rightarrow D^{-} l^{+} \nu$ with $D^{-} \rightarrow K_{L}^{0} l^{-}$; and $B^{0} \rightarrow \rho^{-} l^{+} \nu$, with the $\pi^{0}$ from the $\rho$ decay treated, if detected, as part of the tag-candidate. For the latter calculation, we assumed isotropic $\rho$ decay, and a branching ratio four times that for $B^{0} \rightarrow \pi^{-} l^{+} \nu$. The expected backgrounds are $2 \%$ and $4 \%$, respectively; neither process shows a peak in $m_{\nu}{ }^{2}$, although the first process does show a peak in $p^{*}$. Hence neither poses special problems.

These calculations ignore the effect of beam energy spread, which, due to the low $Q$-value for the $\Upsilon(4 \mathrm{~S})$ decay, can significantly broaden the $p^{*}$ distribution. (There is no significant effect on the reconstructed $m_{\nu}{ }^{2}$.) For a $5 \mathrm{MeV}$ r.m.s. spread in the ovcrall c.m. energy (the result, for example, of indi-
Figure 6.13 Distribution of reconstructed $m_{\nu}{ }^{2}$ for $B^{0} \rightarrow \pi^{-} l^{+} \nu$ after cuts.

The detection efficiency for $B^{0} \rightarrow \pi^{-} l^{+} \nu$ can be $\approx 6 \%$, while that for $B \bar{B}$ background is $\sim 0.001 \%$.

Beam energy spread has an important effect on the width of the $p^{*}$ distribution. 
We should achieve a signalto-background ratio for $B \rightarrow$ $\pi l^{+} \nu$ of $\sim 1: 1$, with the background subtracted statistically.

$B \rightarrow \rho l^{+} \nu$ at an asymmetric $B$ factory: vertex information will be of considerable help. vidual beam energy fractional spreads of $6.7 \times 10^{-4}$ r.m.s.), achieving the same $\epsilon=0.059$ requires increasing the accepted $p^{*}$ range - and hence most of the backgrounds - by a factor of 1.55 . (The $p^{*}$ cuts become \pm 70 instead of $\pm 45 \mathrm{MeV}$.) However, it is highly likely that more sophisticated eventrecognition cuts, relying primarily on vertex analysis but also on kinematics, can gain back this factor and more.

Thus the overall signal-to-background level is expected to be at least $\sim 1: 1$, with the background separable statistically. We conclude that the initial experiment would provide a good first measurement of $B \rightarrow \pi l^{+} \nu$. With the full data set of $300 \mathrm{fb}^{-1}$ we could measure the yield separately for $l=e$ and $l=\mu$ in each of ten $q^{2}$ bins with $10 \%$ statistical precision.

While we have not carried out a Monte Carlo study of $B \rightarrow \rho l^{+} \nu$, a few remarks are in order. The easiest way to measure this will be in the charge state $B^{+} \rightarrow \rho^{0} l^{+} \nu$, for which all the detectable particles are charged. If the branching ratio is indeed significantly larger than that for $B \rightarrow \pi l^{+} \nu$, then measuring it will be less demanding. However, as already pointed out, using this process to assess models for the form factors and to believably extract $\left|V_{u b}\right|$ requires measuring not only the $q^{2}$ dependence, but also joint angular distributions. This will require higher statistics and lower background than does the $B^{0} \rightarrow \pi^{-} l^{+} \nu$ measurement. Fortunately, the requirements that the $\pi^{+}$and $\pi^{-}$form a $\rho^{0}$ and that all three detected decay particles come from a common vertex should help considerably. In fact, we have been informed that a DESY asymmetric collider study concluded that vertex cuts are powerful enough to allow measuring the $B^{+} \rightarrow \rho^{0} l^{+} \nu$ branching ratio even without tagging (and therefore without $p^{*}$ cuts). Of course, a detailed simulation is needed to assess how well one can do, particularly for the separation of the form factors. But in any case, by allowing one to combine all of these tools, an asymmetric collider should provide a significant edge in understanding this decay. 
$\underline{\text { Exclusive Hadronic Decays }}$

$\underline{B^{0} \rightarrow \pi^{+} \pi^{-}}$

The branching fraction for the decay $B^{\circ} \rightarrow \pi^{+} \pi^{-}$is predicted by Bauer, Stech and Wirbel ${ }^{8}$ to be $2.1 \times 10^{-3} \times$ $\left(V_{u b} / V_{c b}\right)^{2}$. If $V_{u b} / V_{c b}$ is about 0.1 , as recent semi-leptonic decay results from CLEO and ARGUS suggest, this would imply $\operatorname{Br}\left(B^{\circ} \rightarrow \pi^{+} \pi^{-}\right) \sim 2 \times 10^{-5}$. In an inital data sample of $30 \mathrm{fb}^{-1}$ this represents over 1000 produced events of this type.

A branching ratio of this order of magnitude is too small to have been detected in any of the currently available $B \bar{B}$ data samples; the best upper limit ${ }^{17}$ on the branching ratio from CLEO is $0.9 \times 10^{-4}$. However, in the CLEO data sample of $242,000 B \bar{B}$ events there is already a significant background of the order of 0.4 events/ $\mathrm{MeV}$ from continuum $q \bar{q}$ events. Extrapolating their observed background level to a data sample twenty-five times larger, they will observe 100 background events in a signal region of $10 \mathrm{MeV}$ in beamenergy constrained mass, compared to a signal of 50 events assuming a $2 \times 10^{-5}$ branching ratio and using their quoted efficiency of $45 \%$. It will be necessary to do much better in order to study CP-violation in this channel. We have investigated ways to further suppress the backgrounds at an asymmetric collider, and found that vertex cuts and improved particle identification can significantly improve the signal to background while retaining a reasonable detection efficiency.

The decay mode $B^{\circ} \rightarrow \pi^{+} \pi^{-}$was studied using an asymmetric version of LUND 6.3, using beam energies of $9 \mathrm{GeV}$ on $3.11 \mathrm{GeV}$. One $B$ was forced to decay to $\pi^{+} \pi^{-}$while the other $B$ decayed to a cocktail of final states. The detector response was simulated as described in Chapter 3; only those tracks within the detector acceptance were considered. The simple nature of the two-body final state made it very easy to reconstruct and identify. In Figure 6.14, the invariant mass of all opposite-charged tracks is shown; the $B^{\circ} \rightarrow \pi^{+} \pi^{-}$signal is very clear and there is no combinatoric background from other $B$ decays. A Gaussian fit to the data yields a mass of $5.277 \mathrm{GeV}$ and $\sigma=23 \mathrm{MeV}$. Assuming a detector which
Continuum $q \bar{q}$ backgrounds are already limiting present searches for $B^{\circ} \rightarrow \pi^{+} \pi^{-}$.

The detector acceptance for $B^{\circ} \rightarrow \pi^{+} \pi^{-}$is $86 \%$ for an asymmetric machine of 9 on $3.11 \mathrm{GeV}$. 
Figure 6.14 Two-pion invariant mass distribution for reconstructed $B^{\circ} \rightarrow \pi^{+} \pi^{-}$decays.

Figure 6.15 Invariant mass distribution for all opposite sign charged tracks in continuum $q \bar{q}$ events. extends down to $|\cos \theta|>.95$, the geometrical acceptance for $B^{\circ} \rightarrow \pi^{+} \pi^{-}$is very high, approximately $86 \%$.
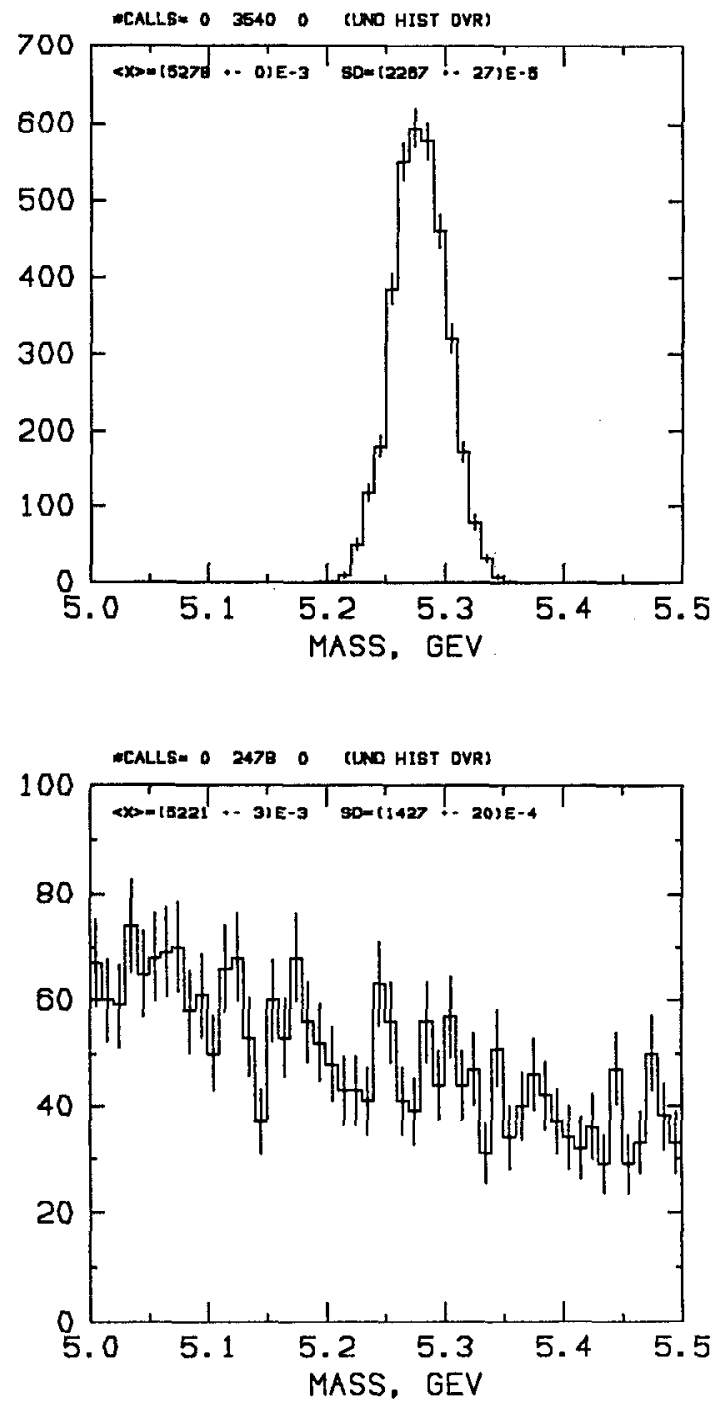

To investigate the background due to continuum $q \bar{q}$ events, $u \bar{u}, d \bar{d}, s \bar{s}$ and $c \bar{c}$ events were generated with standard couplings again using LUND 6.3; the invariant mass distribution of all opposite charge tracks is shown in Figure 6.15. In the region of the $B$ mass, from 5.0 to $5.5 \mathrm{GeV}$, the background is linear and slowly falling.- The fraction of continuum events which contribute in this region is $0.8 \%$. We have also investigated possible backgrounds from other low-multiplicity $B$ decays, such as $B \rightarrow D K$, where $D \rightarrow K \pi$. However no de- 
tectable backgrounds of this type were found, even when both $B$ 's decay to such final states. We conclude that the dominant source of background is from continuum $q \bar{q}$ production.

To reduce the continuum background we imposed two kinematic cuts on the two-pion systcm, after boosting back to the center-of-mass frame. First, the vector sum of the pion momenta is required to be between 250 and $410 \mathrm{MeV}$. The signal and background distributions for this quantity are shown in Figures 6.16 and 6.17. The distributions are smeared by an assumed center-of-mass spread of $5 \mathrm{MeV}$ as well as by detector resolution. This cul reduces the background by a factor of $2.6 \times 10^{-2}$, with $92 \%$ efficiency for the signal. Note that this cut is equivalent to restricting the beam-energy constrained mass, $m_{b c}=\sqrt{E_{b}^{2}-p_{c m}^{2}}$, to be within $\pm 5 \mathrm{MeV}$ of the $B$ mass.

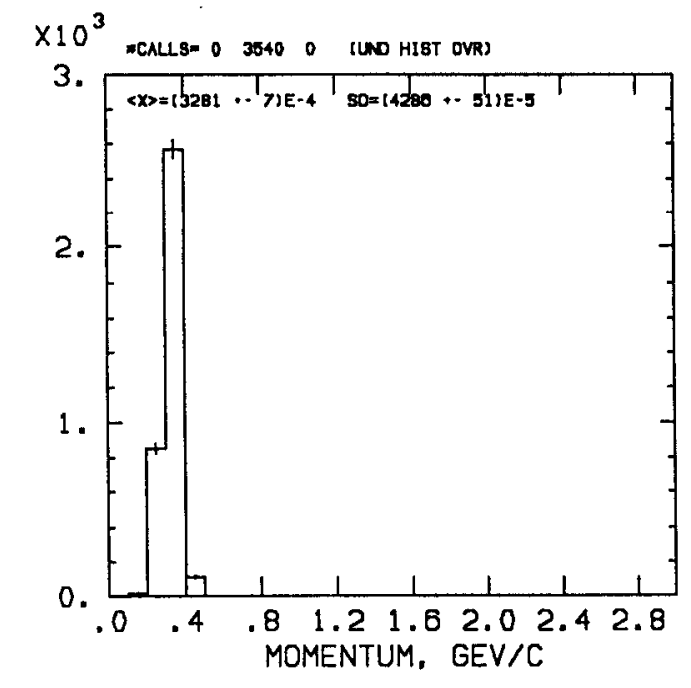

Two kinematic cuts are used to reduce the continuum $q \bar{q}$ background.

Figure 6.16 Momentum of candidate two-pion system in the center-of-mass frame for $B^{\circ} \rightarrow \pi^{+} \pi^{-}$events. 
Figure 6.17 Momentum of candiate two-pion system in center of mass frame in continuum $q \bar{q}$ events with an invariant $\pi \pi$ mass from $5-5.5$ $\mathrm{GeV} / \mathrm{c}^{2}$
Figure 6.18 Cosine of the angle between the sphericity axis and one of the candidate pions in the center of mass frame, for $B^{\circ} \rightarrow \pi^{+} \pi^{-}$events.

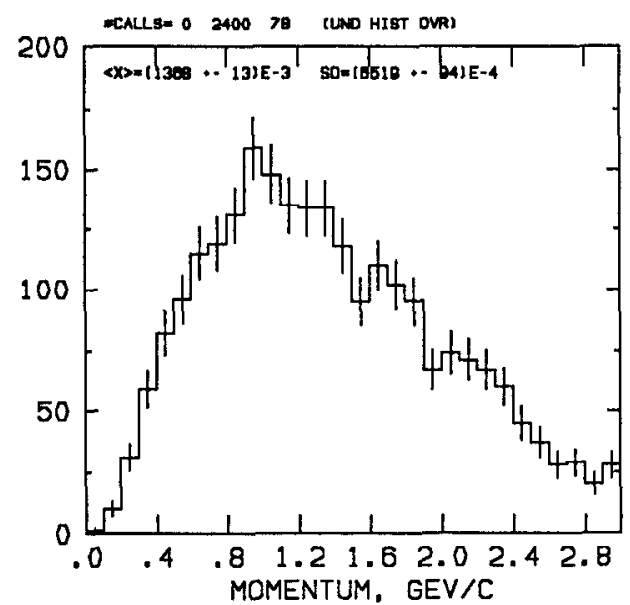

Second, additional continuum reduction is achieved by exploiting the difference in event topologies. $B \bar{B}$ events decay isotropically in the center of mass frame while continuum events are more jet-like. Therefore the angle between either of the $\pi$ 's and the sphericity axis, computed in the center-ofmass frame after removing both candidate $\pi$ 's, will tend to be flat for signal events and peaked forward and backward for background. These angular distributions are shown in Figures 6.18 and 6.19 ; requiring $\left|\cos \theta_{s p h}\right|<0.7$ rejects $90 \%$ of the background and has an efficiency of $70 \%$ for the signal.

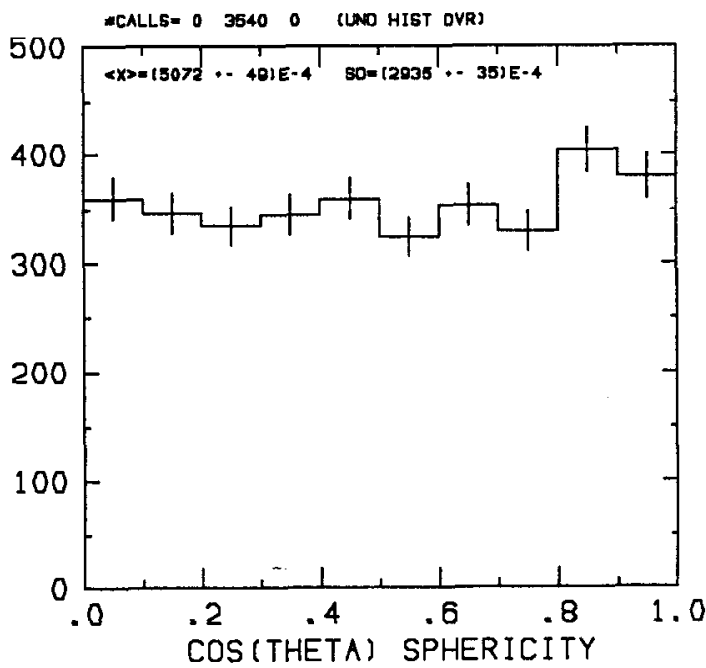




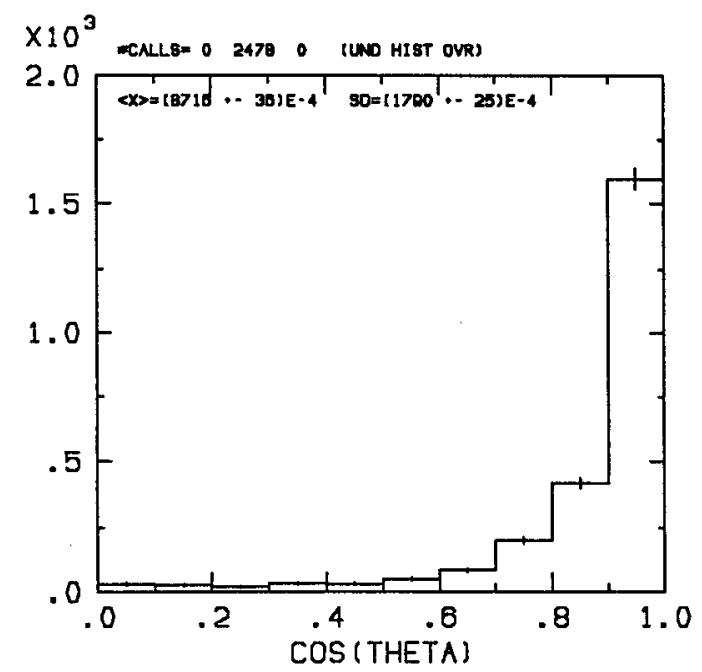

At this stage of the analysis we observe a continuum background level of $1.4 \times 10^{-7}$ events $/ \mathrm{MeV} / B \bar{B}$ pair in a signal region of $92 \mathrm{MeV}$ in unconstrained mass i.e., within $\pm 2 \sigma$ of the $B$ mass. This is consistent with CLEO's observed background level of $1.6 \times 10^{-6}$ events $/ \mathrm{MeV} / B \bar{B}$ pair in a signal region of $10 \mathrm{MeV}$ in beam-constrained mass, which has a resolution an order of magnitude smaller than unconstrained mass. We have chosen to cut on $p_{c m}$ and plot unconstrained mass because it leaves the background distribution flat in invariant mass.

We now apply the additional cuts, unique to an asymmetric machine, which will greatly improve the signal to background level. We begin by requiring that both pions form a vertex with a probability greater than $5 \%$. This cut rejects backgrounds in which the tracks come from different vertices, such as $e^{+} e^{-} \rightarrow c \bar{c}$, where one of the tracks comes from a $D$ decay and the other comes from the other $D$ decay or from the primary vertex. However, because most of the continuum background comes from $u \bar{u}$ events, only $14 \%$ of the background sample is rejected. The efficiency for the signal events is $95 \%$. See Figures 6.20 and 6.21 for vertex probability distributions for signal and background, respectively.
Figure 6.19 Cosine of the angle between the sphericity axis and one of the candidate pions in the center of mass frame, for $q \bar{q}$ events.

Comparison is made to observed background levels in the CLEO analysis after applying standard kinematic cuts.

Vertex cuts provide additional background rejection. 
Figure 6.20 Vertex probability distribution for reconstructed $B^{\circ} \rightarrow \pi^{+} \pi^{-}$decays.

Figure 6.21 Vertex probability distribution for background pairs from continuum events.
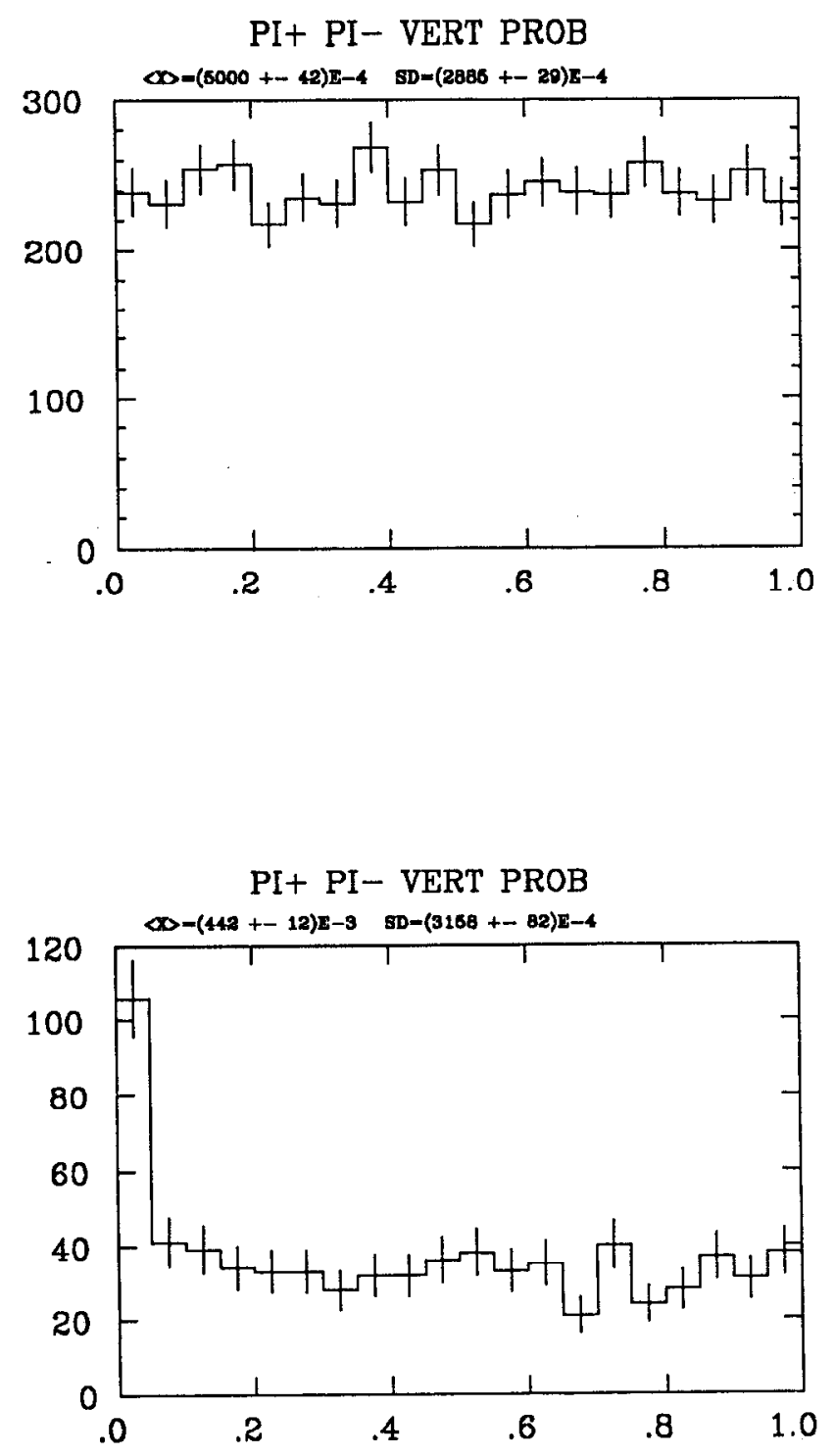


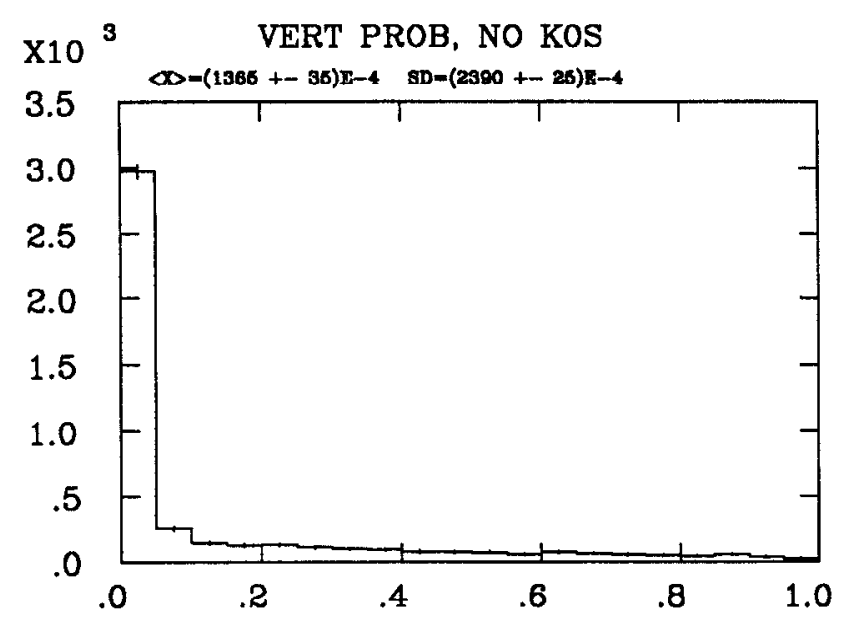

The best rejection of continuum actually comes from the vertex probability distribution for all tracks in the event fit to a common vertex. In this distribution, we excluded all tracks coming from $K_{s}^{0}$ decay. This is a reasonable approximation: although we will have less than perfect $K_{s}^{0}$ rejection due to acceptance, this will be compensated by additional rejection of tracks from hyperon decays, which we have not excluded from the vertex fit in this study. The vertex probability distribution for the hypothesis that all tracks (except for those from $K_{s}^{0}$ decays) come from a common vertex $\left(P_{A L L}\right)$ is shown for our signal sample in Figure 6.22 and for the continuum background events in Figure 6.23. In both plots, the events in which the $B^{0}$ candidate pair failed the $P_{\pi^{+} \pi^{-}}$requirement have been omitted. It is obvious from these plots that there is a much higher fraction of events with $P_{A L L}<0.05$ in the signal sample than in the continuum background sample. If we require both $P_{\pi^{+} \pi^{-}}>0.05$ and $P_{A L L}<0.05$, the efficiency is $65 \%$ for the signal, while $84 \%$ of the remaining continuum background events are rejected. The combined efficiency of both vertex cuts is $62 \%$ for signal events and $14 \%$ for continuum background events, or an improvement in the signal to background ratio $(S / B)$ of 4.4 . This corresponds to a substantial reduction in the integrated luminosity necessary for this study.
Figure 6.22 Vertex probability distribution for signal events with $P_{\pi^{+}-}>0.05$, for all tracks (except those from $K_{s}^{0}$ decay) fit to a common vertex.

The signal-to-background ratio is improved by a factor of 4.4 by appropriate vertex cuts. 
Figure 6.23 Vertex probability distribution for continuum background events with $P_{\pi^{+} \pi^{-}}>0.05$, for all tracks, except those from $K_{s}^{0}$ decay, fit to a common vertex.

Particle identification using a CRID provides additional continuum rejection, as well as separating $B^{\circ} \rightarrow \pi^{+} \pi^{-}$from other rare decays such as $B^{\circ} \rightarrow$ $K^{+} \pi^{-}$and $B^{\circ} \rightarrow K^{+} K^{-}$.

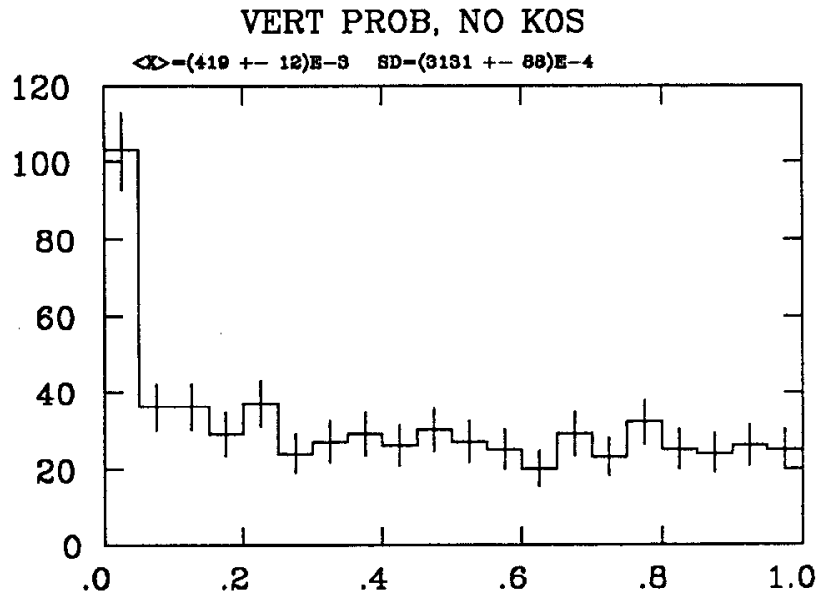

Finally, we impose particle identification requirements on the event. With the particle identification capabilities of a Cerenkov ring-imaging detector (CRID) we will be able to unambiguously separate pions from kaons even in the momentum range from 2 to $4 \mathrm{GeV} / \mathrm{c}$ which is populated by twobody $B$ decays. We require both tracks be within $\pm 2 \sigma$ of the expected CRID radius for a pion, and greater than $2 \sigma$ away from that expected for a kaon. This rejects $99.3 \%$ of all kaons with $91 \%$ efficiency for acceptance of both pions. The particle identification requirement rejects $70 \%$ of the continuum background events; this reflects the fact that protons and kaons form a significant fraction $(\sim 40 \%)$ of all charged particles in $q \bar{q}$ events which are consistent with the $B$ mass. In additon to reducing the dominant source of background, another important benefit of the CRID is that we can cleanly separate the process $B^{\circ} \rightarrow \pi^{+} \pi^{-}$from $B^{\circ} \rightarrow K^{+} \pi^{-}$and $B^{\circ} \rightarrow K^{+} K^{-}$, which are interesting decays in their own right but which would dilute the $\mathrm{CP}$-violating asymmetry measurement in the $B^{\circ} \rightarrow \pi^{+} \pi^{-}$system.

In conclusion, we have demonstrated that the process $B^{\circ} \rightarrow \pi^{+} \pi^{-}$can be observed at an asymmetric $B$ factory running on the $\Upsilon(4 \mathrm{~S})$ with a total detection efficiency of $30 \%$. The expected background from continuum $q \bar{q}$ production is $4.9 \times 10^{-7}$ background event $B \bar{B}$ event in a $\pm 2 \sigma$ signal region of $92 \mathrm{MeV}$ around the $B$ mass. (This assumes that all cuts on the continuum events are orthogonal; we have tested 
that this assumption is reasonably accurate by taking all cuts pairwise and observing that the product of the rejection factors individually is similar to the case when they are applied sequentially.) Thus we will be able to detect the processs $B^{\circ} \rightarrow \pi^{+} \pi^{-}$if it occurs at the level of a few times $10^{-5}$ with a signal-to-noise ratio of $\sim 12$. Without vertex cuts and the excellent particle identification capabilities of a CRID, the expected signal to background is over an order of magnitude worse.

$\underline{B^{0} \rightarrow \rho^{\circ} \rho^{\circ}}$

The process $\bar{B}^{0} \rightarrow \rho^{0} \rho^{0}$ proceeds via a color-suppressed spectator decay of a $b$ quark to a $u$ quark: it is thus sensitive to $V_{u b}$. with a predicted ${ }^{8}$ branching ratio $\bar{B}^{0} \rightarrow \rho^{0} \rho^{0}$ of about $10^{-4} \times\left|V_{u b} / V_{c b}\right|^{2}$. Recent CLEO and ARGUS measurements suggest that $\left|V_{u b} / V_{c b}\right|$ is about 0.1 , yielding a branching fraction of $10^{-6}$. The upper limit on $\operatorname{BR}\left(\bar{B}^{0} \rightarrow \rho^{0} \rho^{0}\right)$ from CLEO measurements $^{17}$ for a sample of $264,000 B$ mesons is more than 2 orders of magnitude larger than this prediction. This clearly requires large increases in the luminosity as well as major improvements in background rejection techniques. These background-rejection improvements can best be realized at an asymmetric $B$ factory.

The process under study was investigated by Monte Carlo simulations, using the Lund 6.3 model. One of the $\bar{B}^{0}$ mesons was forced to decay into $\rho^{0} \rho^{0}$, the other was free to decay into anything. Since the $\rho^{0}$ decays with $100 \%$ into $\pi^{+} \pi^{-}$, $\bar{B}^{0} \rightarrow \rho^{0} \rho^{0}$ events are reconstructed by requiring two $\pi^{+} \pi^{-}$ pairs with invariant masses between 0.66 and $1.1 \mathrm{GeV} / c^{2}$ to account for the broad $\rho^{0}$ width (Figure 6.24 , left). This cut was chosen to be asymmetric about the nominal mass, in order to keep the signal efficiency as high as possible and to reduce background, which decreases with increasing mass. The invariant mass of two $\rho^{0}$ candidates is then required to be consistent with the $\bar{B}^{0}$ mass (Figure 6.24 , right).
Current experimental upper limits on $B R\left(\bar{B}^{0} \rightarrow \rho^{0} \rho^{0}\right)$ are two orders of magnitude larger than theoretical predictions. 
Figure 6.24 $\rho^{0}$ mass (left) and $B$ mass (right) distributions for reconstructed $\bar{B}^{0} \rightarrow \rho^{0} \rho^{0}$ events.

Kinematic cuts reduce the continuum background.

Figure 6.25 Momentum distribution of the four-pionsystem in the $\Upsilon(4 S)$ rest frame for reconstructed $\vec{B}^{0} \rightarrow \rho^{0} \rho^{0}$ events (left) and continuum background (right).
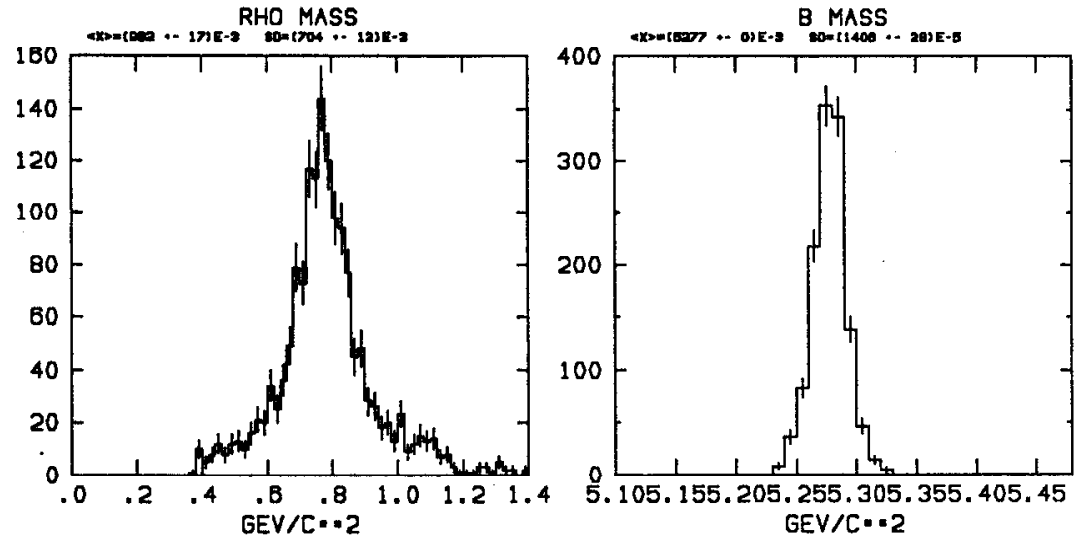

There is a substantial combinatorial background from $b \bar{b}$ and continuum events, which have a high charged pion multiplicity. To reduce this background, we calculate the momentum of the four $\pi$ 's in the $\Upsilon(4 S)$ rest frame and require it to be between 0.1 and $0.42 \mathrm{GeV} / \mathrm{c}$ (Figure 6.25), using a enter-of-mass energy spread of $5 \mathrm{MeV}$. This asymmetric cut alone rejects continuum and $b \bar{b}$ background with an efficiency of better than 1000:1.
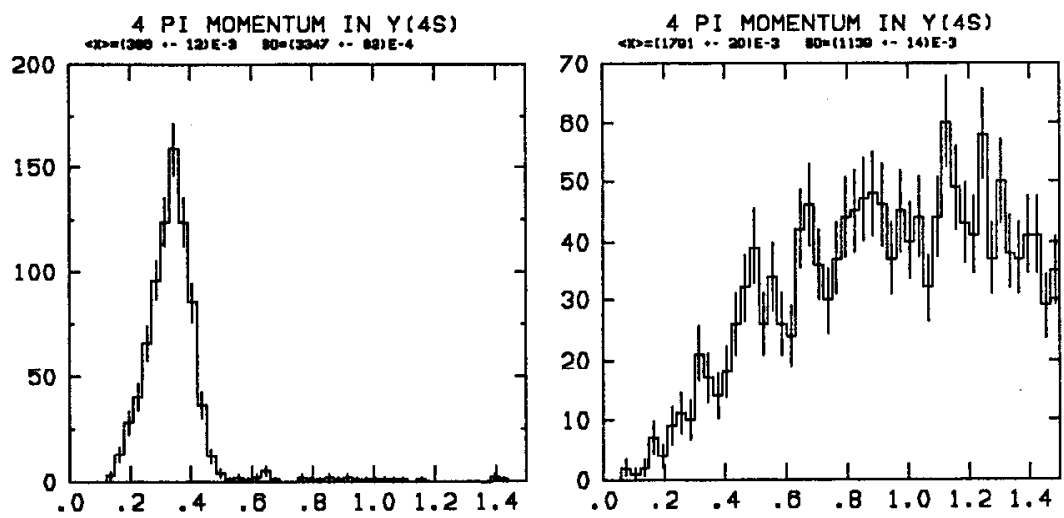

Further background rejection is accomplished by constraining the four $\pi$ 's to a common vertex, thereby taking advantage of the boost provided by the energy asymmetry of the collider. The vertexing probability $P_{4 \pi}$ is a flat distribution if the tracks come from the same vertex, but is enhanced near 
zero probability for background events, i.e. when the four $\pi$ 's of two $\rho^{0}$ candidates come from different vertices. The requirement, $P_{4 \pi} \geq 0.05$, is thus $95 \%$ efficient for the signal but rejects about $34 \%$ of the background events. This background suppression is better than in the $\bar{B}^{0} \rightarrow \pi^{+} \pi^{-}$case, due to the fact that four pions are constrained to come from one vertex.

Additional background rejection is obtained by making use of the fact that the tracks of the light quark continuum events $(d \bar{d}, u \bar{u}$ and $s \bar{s})$ form a common primary vertex, if tracks from $K_{s}^{0}$ and hyperon decays are excluded. Here we used all charged tracks to calculate the vertexing probability, $P_{\text {all }}$. In fact, the reconstruction of $K_{s}^{0}$ did not change the result significantly, since most events which pass this cut are due to secondary vertices from $D$ decay. With the requirement $P_{a l l} \leq 0.05$, we exclude events where all tracks emerge from one vertex. This cut reduces the background by a factor of 4 and $\bar{B}^{0} \rightarrow \rho^{0} \rho^{0}$ events pass with a $67 \%$ efficiency. Both vertex cuts combined lead to a background to signal rejection factor of 4 , which is somewhat smaller than that described in the section on the $\bar{B}^{0} \rightarrow \pi^{+} \pi^{-}$decay.

Since the B's are produced nearly at rest in the center of mass system, events are rather spherical. This can be used to reduce background even further. Omitting the two $\rho^{0}$ candidates, we calculate the sphericity of the remaining event in the $\Upsilon(4 S)$ rest frame. If $\alpha$ denotes the angle between the sphericity axis and either $\rho^{0}$ in the $\rho \rho$ c.m. frame, the $\cos \alpha$ distribution for $\bar{B}^{0} \rightarrow \rho^{0} \rho^{0}$ events is nearly flat, as expected. In contrast, the distribution for $\rho^{0}$ candidates from continuum events peaks at forward and backward angles. We required $|\cos \alpha|$ to be less than 0.8 , thereby reducing the continuum background by a factor of about 3 with $80 \%$ efficiency for $\bar{B}^{0} \rightarrow \rho^{0} \rho^{0}$ events (Figure 6.26).
Vertex cuts provide additional background rejection. 
Figure $\quad 6.26 \quad|\cos \alpha|$ distribution for reconstructed $\bar{B}^{0} \rightarrow \rho^{0} \rho^{0}$ events (left) and for continuum background events (right).

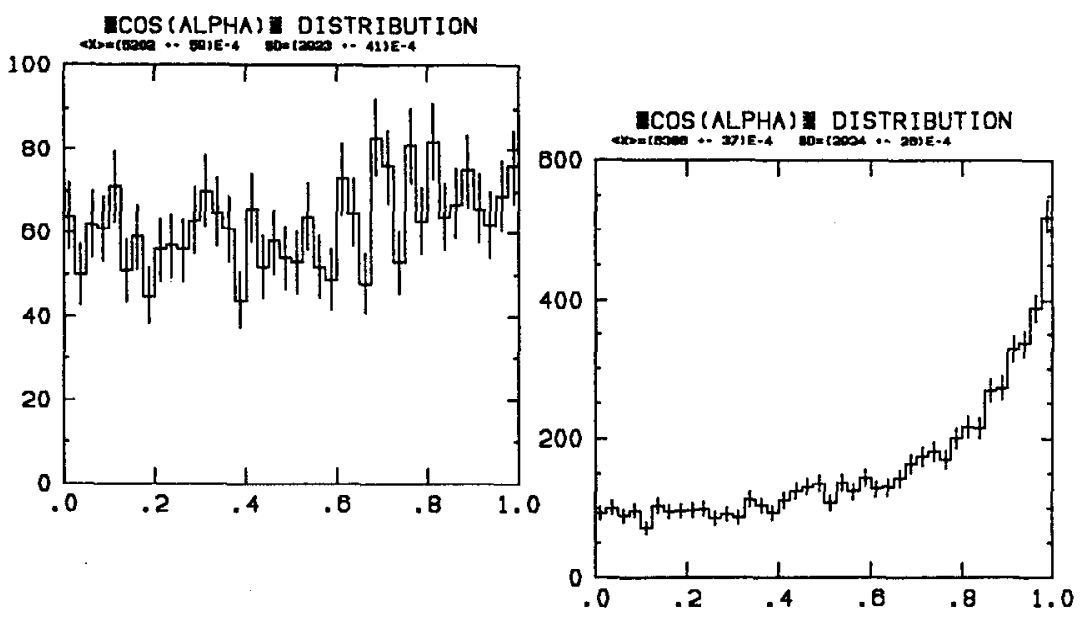

In conclusion, we find an overall reconstruction efficiency for $\bar{B}^{0} \rightarrow \rho^{0} \rho^{0}$ events of $28 \%$, which includes the detector efficiency. We simulated a sample of $2.7 \times 10^{5}$ continuum events, which, after all cuts, resulted in 0.002 background events $/ \mathrm{MeV}$ in the $B$ mass region. The distribution for the $B$ candidates from this background peaks near twice the $\rho^{0}$ mass and falls sharply off towards higher invariant mass. This is understood from the two constraints on the $\rho^{0}$ mass and the low momentum of the $4 \pi$ 's in the $\Upsilon(4 S)$ frame. A similar sample of $b \bar{b}$ events yields a suppression factor which is better by an order of magnitude. From these studies, we find an encouraging signal-to-background ratio of about 1:2.5, assuming a $\bar{B}^{0} \rightarrow \rho^{0} \rho^{0}$ branching ratio of $10^{-6}$ and a signal region of $\pm 15 \mathrm{MeV}$ about the nominal $B$ mass (this is equivalent to $\mathrm{a} \pm 1 \sigma$ cut in mass). In Figure 6.27 we show the signal and background one would expect for a sample of $10^{8} B \bar{B}$ events $\left(120 \mathrm{fb}^{-1}\right)$. The observation of this decay mode is thus clearly possible, thereby providing a test of the BSW model as well as an independent method of determining $\left|V_{u b}\right|$. 


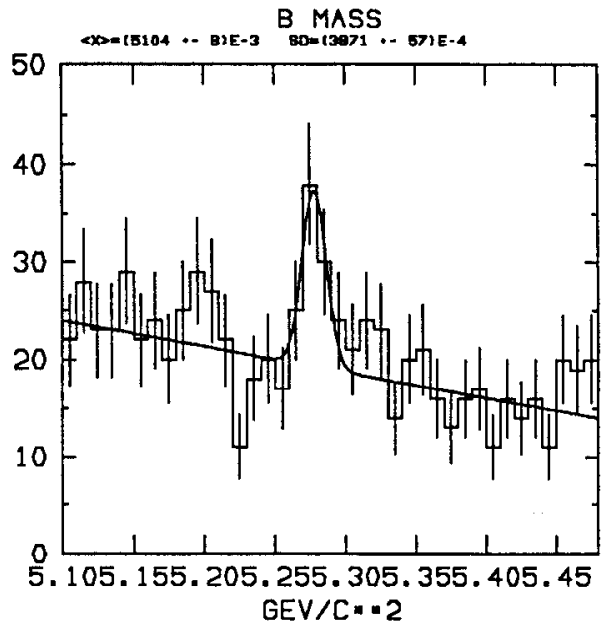

$\underline{B^{0} \rightarrow \omega^{0} \pi^{0}}$

The branching ratio for the $\mathrm{CP}$ eigenstate decay $B^{0} \rightarrow$ $\omega^{0} \pi^{0}$ could be as large as ${ }^{8} 1 \times 10^{-5}$. We have studied the possibility of measuring the branching ratio for this reaction in the initial data sample of $30 \mathrm{fb}^{-1}$ preparatory to a possible measurement of the CP asymmetry for this decay in the "program" sample of $300 \mathrm{fb}^{-1}$. We have explored the reconstruction of this "difficult" final state which includes neutrals as a prototype for other decays which include $\omega^{0}$ mesons, such as $B_{s}^{0} \rightarrow \omega^{0} K_{s}^{0}$, which could show a CP asymmetry different from that in $B_{s}^{0} \rightarrow \rho^{0} K_{s}^{0}$ decay.

We generated $10,000 B^{0} \bar{B}^{0}$ events in which one of the $B$ mesons decayed into $\omega^{0} \pi^{0}$. We then formed all two-photon invariant mass combinations; the result is shown in Figure 6.28. There is a clear signal at the $\pi^{0}$ mass, defined as 1.25 to $1.45 \mathrm{GeV} / \mathrm{c}^{2}$. We then combined these $\pi^{0}$ candidates with all pairs of oppositely charged tracks in the event, and found an unambiguous signal for $\omega^{0} \rightarrow \pi^{+} \pi^{-} \pi^{0}$ decay (see Figure 6.29).
Figure 6.27 Expected signal and background for a sample of $10^{8} B \bar{B}$ events and a $\bar{B}^{0} \rightarrow$ $\rho^{0} \rho^{0}$ branching ratio of $10^{-6}$.
The decay $B^{\circ} \rightarrow \omega^{\circ} \pi^{\circ}$ provides a test of our ability to reconstruct "difficult" modes with neutrals. 
Figure 6.28 Invariant mass distribution for all photon pairs in $10 \mathrm{~K} B^{0} \bar{B}^{0}$ events, where one $B$ decays into $\omega^{0} \pi^{0}$, and the other $B$ decays into the standard cocktail.

Figure 6.29 Invariant mass distribution for all $\pi^{+} \pi^{-} \pi^{0}$ combinations in $10 \mathrm{~K} B^{0} \bar{B}^{0}$ events, where one $B$ decays into $\omega^{0} \pi^{0}$.
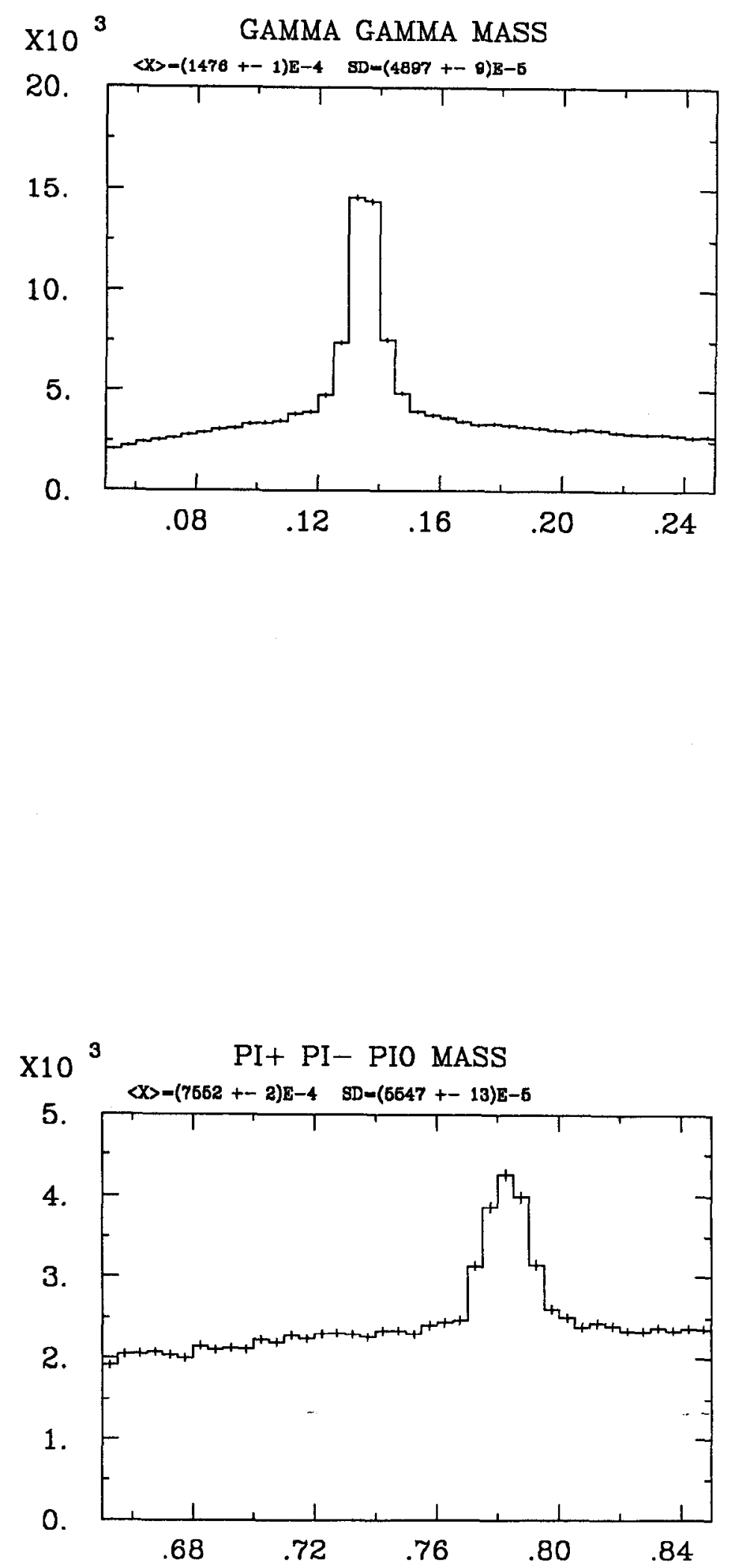


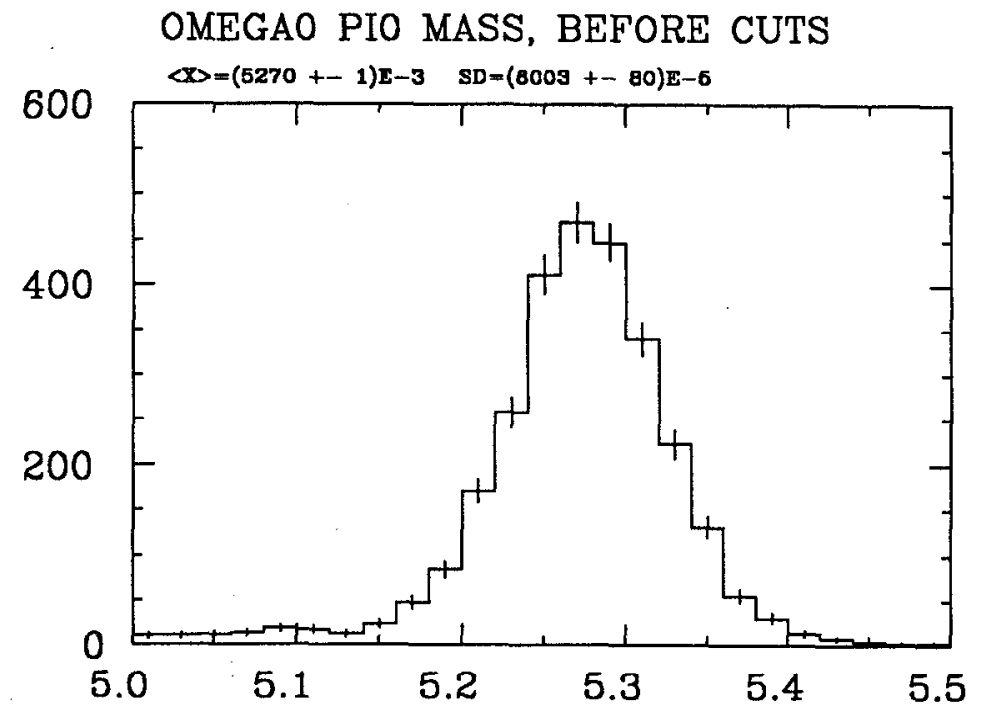

We required the $\pi^{+} \pi^{-} \pi^{0}$ mass be between 0.770 and 0.795 $\mathrm{GeV} / \mathrm{c}^{2}$ to form a candidate $\omega^{0}$ decay. We then combined the $\omega^{0}$ candidates with all remaining $\pi^{0}$ candidates in the event and plotted the $\omega^{0} \pi^{0}$ invariant mass (Figure 6.30). There is a clear signal for the decay $B^{0} \rightarrow \omega^{0} \pi^{0}$. If we define our $B^{0}$ signal to be all $\omega^{0} \pi^{0}$ combinations with mass between 5.16 and $5.40 \mathrm{GeV} / \mathrm{c}^{2}$, there are 2669 entries, corresponding to a reconstruction efficiency of 0.27 . For our initial sample, this would correspond to 80 reconstructed decays.

There is, however, significant background to this reaction. We have generated $331 \mathrm{~K}$ standard $b \bar{b}$ events and $500 \mathrm{~K}$ continuum events, and find 3 and 290 events, respectively, which pass these cuts. These would correspond to backgrounds of 272 events from $\bar{B}$ production and 69600 events from $q \bar{q}$ production compared to our signal of 80 events.
Figure 6.30 Invariant mass distribution for all $\omega^{0} \pi^{0}$ pairs in $10 \mathrm{~K} B^{0} \bar{B}^{0}$ events, where one $B$ decays into $\omega^{0} \pi^{0}$.
The reconstruction efficiency after making nominal mass cuts is $27 \%$. 
Figure 6.31 Center of mass momentum distribution for all $\omega^{0} \pi^{0}$ pairs with mass between 5.0 and $5.5 \mathrm{GeV} / \mathrm{c}^{2}$ in $10 \mathrm{~K}$ $B^{0} \bar{B}^{0}$ events, where one $B$ decays into $\omega^{0} \pi^{0}$.

A set of simple cuts is sufficient to suppress continuum backgrounds.

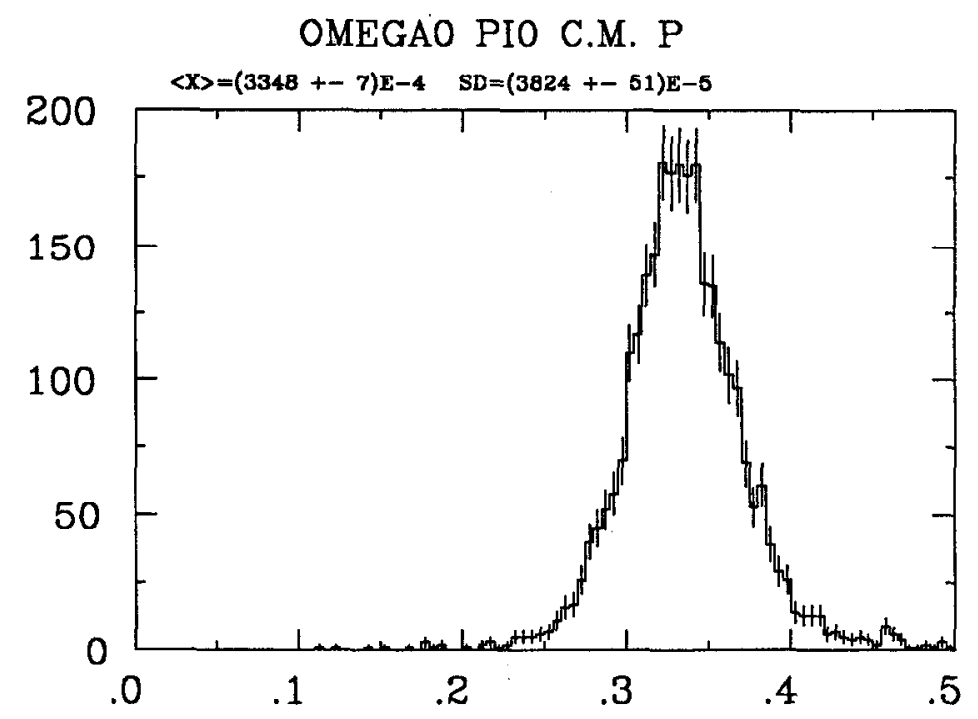

We employed four cuts to suppress the background. Figure 6.31 shows the center-of-mass momentum distribution for the properly reconstructed $\omega^{0} \pi^{0}$ decays. We required that this momentum be between 0.27 and $0.40 \mathrm{GeV} / \mathrm{c}$. We also plotted the angle in the center of mass frame between the $\pi^{0}$ from the $B^{0}$ decay and the sphericity axis of all tracks not coming from the $B^{0}$ decay candidate $\left(\cos \theta_{s}\right)$. We observed that the distribution for real $B^{0}$ decay is approximately flat, while the distribution for continuum candidates is strongly peaked at $\left|\cos \theta_{s}\right|=1$. We used the same cut as CLEO, $\left|\cos \theta_{s}\right|<0.7$. We also used the same two vertex probability cuts that we used for the reaction $B^{0} \rightarrow \pi^{+} \pi^{-}$, namely $P_{\pi^{+} \pi^{-}}>0.05$, where $P_{\pi^{+} \pi^{-}}$is the probability that both charged tracks from the candidatc $B^{0}$ decay came from a common vertex, and $P_{A L L}<0.05$, where $P_{A L L}$ is the probability that all tracks in the event (except for those from $K_{s}^{0}$ decay) came from a common vertex. The vertex probability distributions are very similar to those observed in the $B^{0} \rightarrow \pi^{+} \pi^{-}$study. With these cuts, the number of $B^{0} \rightarrow \omega^{0} \pi^{0}$ which we observe in the signal sample is 1053 , or a reconstruction efficiency of $11 \%$. A small amount of combinatorial background to the signal seen in Figure 6.30 at masses less than $5.1 \mathrm{GeV} / \overline{\mathrm{c}}^{2}$ is suppressed. We would expect to see $32 B^{0} \rightarrow \omega^{0} \pi^{0}$ decays in our initial data sample with these cuts.

No events in the normal $b \bar{b}$ or continuum samples pass 
these cuts. If one event had passed the cuts in each of these samples, that would correspond to backgrounds of 91 and 362 events, respectively, or a signal significance of 1.5 . Generating enough events to find the true level of these backgrounds is not justified at this time.

However, for the continuum background, we can measure the effect of each of the cuts separately on the background candidates. If we assume that the cuts are sufficiently orthogonal, we can estimate the total efficiency of the cuts as the product of the individual efficiencies. For the $b \bar{b}$ background, we could not generate enough candidates to even do this, so we will assume that this is not as serious a background as the continuum. For $\omega^{0} \pi^{0}$ candidates in the continuum sample with masses between 5.0 and $5.5 \mathrm{GeV} / \mathrm{c}^{2}$, the net efficiency for events which pass all cuts is $7 \times 10^{-4}$. Multiplying the estimated 69600 continuum background pairs in the initial data sample by this factor results in an estimated background of 50 events. If the $b \bar{b}$ background is not significant, as it appears in these studies, then the significance of the signal for $B^{0} \rightarrow \omega^{0} \pi^{0}$ would be 4.5 if the branching ratio for this reaction is $1 \times 10^{-5}$. Note that without the vertex probability cuts, the significance of the signal would be 2.2 , so the ability to employ vertex probability cuts, which is unique to an asymmetric machine, makes the difference between a questionable and a believable measurement in this case. A more detailed study of the effect of material in the particleidentification system on the two- $\pi^{\circ}$ efficiency, as well as more detailed consideration of backgrounds to this decay mode, is clearly warrented.
Vertex cuts greatly improve the significance of the result. 


\section{6. $b \rightarrow s \gamma:$ Penguin Diagrams}

\section{Introduction}

In the Standard Model flavor-changing neutral currents are forbidden by the GIM mechanism at tree level. They can, however, occur at the one loop level via penguin diagrams, which can give rise to $b \rightarrow s \gamma$ transitions as illustrated in Figure 6.32.

Figure 6.32 Penguin diagram for $b \rightarrow s \gamma$

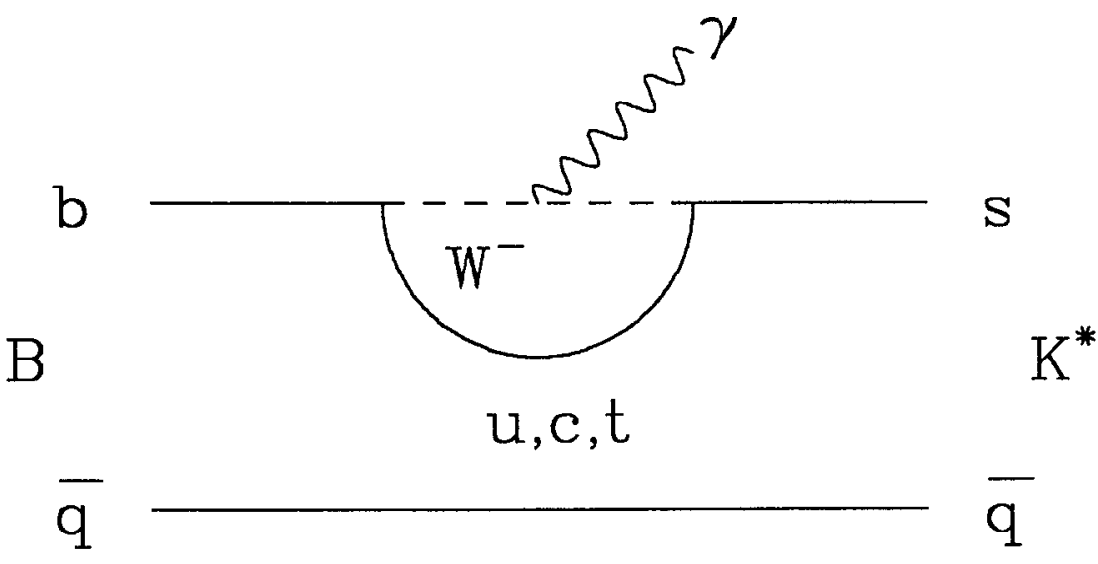

There are a variety of theoretical predictions for inclusive decay modes, as well as for the more experimentally accessible exclusive decay modes such as $K^{*} \gamma$, although these suffer from uncertainties due to hadronic effects. Nevertheless, the measurement of the rate for some of the exclusive decay chanels will give us information on the mass of the top quark as well as on the Higgs sector. The study of these processes is thus sensitive to physics beyond the Standard Model.

We examine herein the reconstruction of a few examples of exclusive $B$ meson decay modes which are produced by penguin diagrams.

\section{$\underline{B \rightarrow K^{*} \gamma}$}

The decay mode $B \rightarrow K^{*} \gamma$ has been studied in a Monte Carlo simulation using the detector simulation described earlier. Only the $K^{*} \rightarrow K^{\mp} \pi^{ \pm}$decay mode has been studied; presumably additional sensitivity could be obtained if one used the neutral $K^{*}$ decay mode. This particular study was 
done using beam energies of 8 and $3.5 \mathrm{GeV}$ and involves fully reconstructing the $B$ final state with positive identification required for the charged $\pi$ and $K$.

A total of $2000 \Upsilon(4 \mathrm{~S}) \rightarrow B^{0} \bar{B}^{0}$ events were generated by the Lund 6.3 MC, with the decay $B^{0} \rightarrow K^{*} \gamma$ and $\bar{B}^{0}$ decay to a cocktail of decay modes. The following set of kinematic cuts provide a clean signal:

1. a photon with a measured energy in the range 1.8 to 3.5 $\mathrm{GeV}$ which does not come from a di-photon pair whose mass is consistent with a $\pi^{\circ}$ or an $\eta$. (It is assumed that the detector can recognize and eliminate interacting neutrons and $K_{L} \mathrm{~s}$. However this assumption is not crucial.)

2. two charged tracks, of opposite charge, whose identification is consistent within three standard deviations with a $\pi$ and $K$, where the particle identification is coming from the CRID system and the invariant mass of the two charged particles is within $100 \mathrm{MeV}$ of the $K^{*}$ mass

3 . the cosine of the angle between the $K^{*}$ and the photon must be less than -0.6 .

4. The " $K^{*} \gamma^{\prime \prime}$ system is transformed into the rest frame of the $\Upsilon(4 \mathrm{~S})$ and the momentum of this system is required to be less than $400 \mathrm{MeV} / \mathrm{c}$ as expected for a parent $B$.

The invariant mass of the $K^{*} \gamma$ system in events satisfying these selection criteria are shown in Figure 6.33. The signal is very clean, only events from the desired topology survive the cuts. The efficiency of the selection criteria is 0.31 .

Backgrounds from continuum events have been checked by applying the same selection criteria to 107,000 events with the standard mix of light quarks. 22 events pass the cuts, but none fall in the $B$ invariant mass bins; the largest invariant mass event is at $4.8 \mathrm{GeV}$. Taking into account the relative normalization of the $\Upsilon(4 \mathrm{~S})$ and the continuum, this amounts to no background events in a sample of 125,000 produced $\bar{B} B$ events. For the "initial" sample, defined in the introduction as $2.510^{7} \bar{B} B$, and a $B^{\circ} \rightarrow K^{*} \gamma$ branching fraction of $210^{-4}$ (as expected for a top mass of $150 \mathrm{GeV}$ ), 1300 signal events are expected. 
Figure 6.33 The $K^{*} \gamma$ invariant mass for the signal.

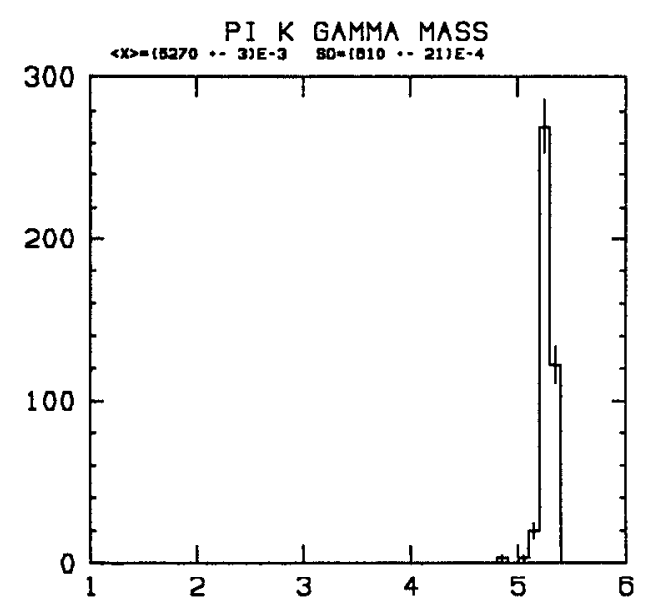

The cuts applied to the events generated by the LUND MC.

In summary then, it appears that a rather simple set of kinematic criteria can easily and efficiently isolate a backgroundfree set of events of the kind $B \rightarrow K^{*} \gamma \cdot 10^{7}$ produced $\bar{B} B$ events will yield on the order of 500 detected events.

\section{$\underline{B \rightarrow \mathrm{Kl}^{+} l^{-}}$}

The $B^{\circ} \rightarrow K_{s}^{o} l^{+} l^{-}$, with $K_{s}^{0} \rightarrow \pi^{+} \pi^{-}$, consists of 4 charged particles, and can thus be completely reconstructed. A total of $20000 \Upsilon(4 S) \rightarrow B^{0} \bar{B}^{0}$ events were generated by the Jund 6.3 MC, with the decay $B^{0} \rightarrow K_{s}^{0} e^{+} e^{-}$and $\bar{B}^{0}$ decay to a cocktail of decay modes. The following requirements give a very clean and unique signal :

1. Two electron candidates (or two muons) must have opposite charge.

2. $K^{0}$ are selected by a two-pion mass cut (0.46-0.54 $\left.\mathrm{GeV}\right)$. No vertex cut is applied ( See Figure 6.34).

3. The four-body mass ( 2 pions in the $K^{0}$ mass range and 2 leptons) must lie in the $B$ peak $(5.25-5.35 \mathrm{GeV})$. See Figure 6.35. 

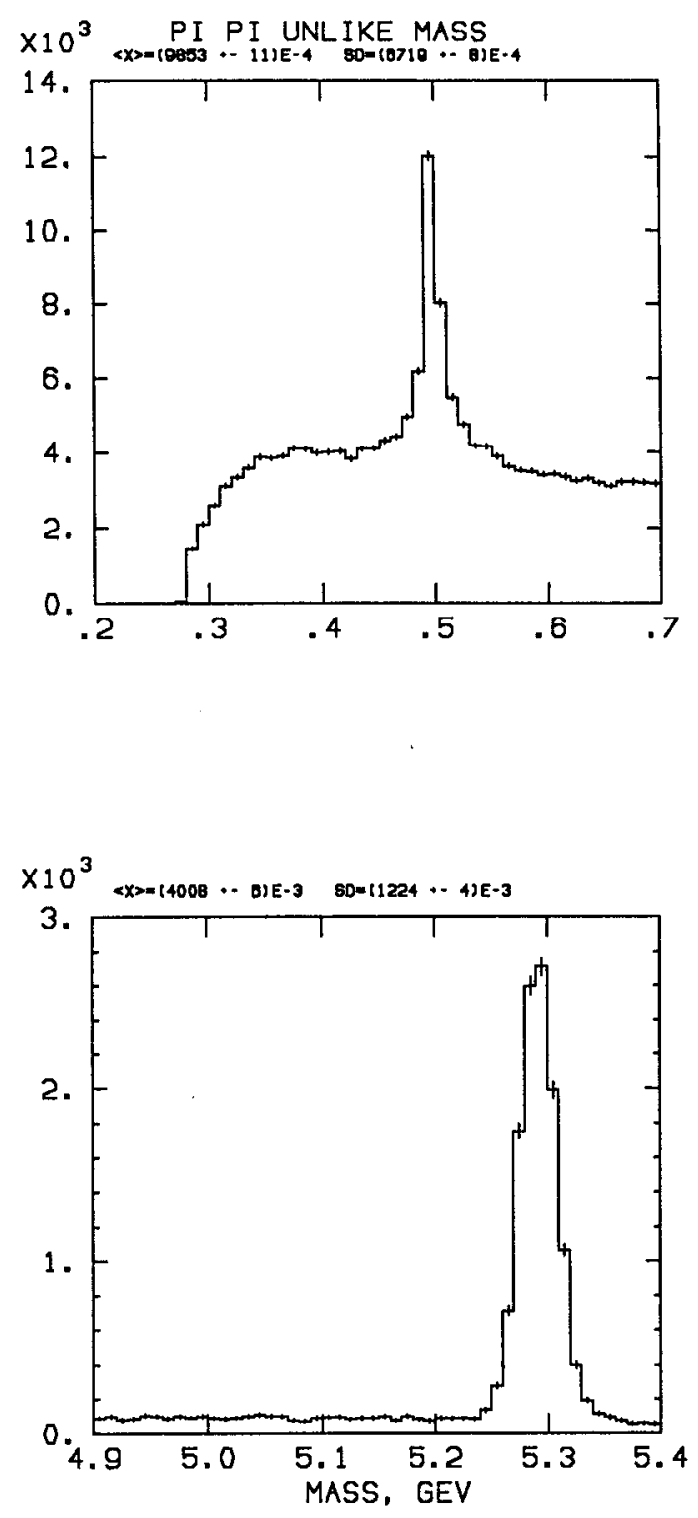

The principal background for this reaction is the reaction $B^{0} \rightarrow K^{0} J / \psi$ with $J / \psi \rightarrow e^{+} e^{-}$, which generates the same four final-state particles. For this reaction $2 \times 10^{4} \mathrm{MC}$ events were generated as above. See Figures 6.36 and 6.37.
Figure 6.34 The two-pion mass spectrum.

Figure 6.35 The four-body mass spectrum: 2 electrons, and 2 pions in the $K^{0}$ mass range. 
Figure 6.36 The twoelectron mass spectrum for the background reaction, without cuts.

Figure 6.37 The electronpair invariant mass distribution for signal events, after all cuts except excluding the $J / \psi$.
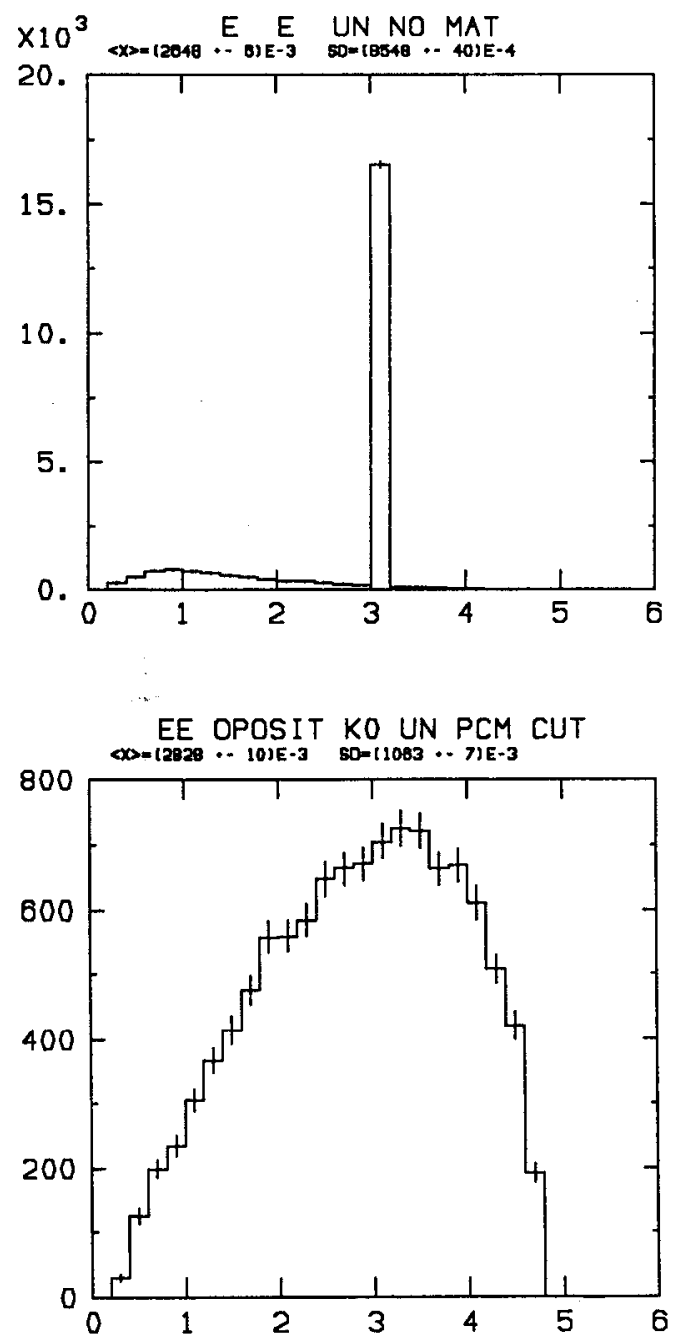

Here the direct signal can be removed by a mass cut on the $e^{+} e^{-}$mass distribution in the region of the $J / \psi, 3.0-3.2$ $\mathrm{GeV}$, leaving only background events to the reaction under study. Since the branching ratio for this reaction can be estimated, we can deduce the potential limits on the reaction under study.

The branching ratio $\mathrm{Br}\left(B^{0}\right.$ or $\left.B^{+} \rightarrow J / \psi+X_{s}\right)$ is $1.12 \%$, where $X_{s}$ is any strangeness +1 system. We can estimate that the $B^{0} \rightarrow K^{0} J / \psi$ fraction, accompanied by $K_{s}^{0} \rightarrow \pi^{+} \pi^{-}$ is $1 / 24$ of this branching ratio. The factors are: neutral $B$ only: $1 / 2 ; K_{s}^{0}$ only: $1 / 2 ; K^{0}$ to charged pions: $2 / 3 ; K$, but not $K^{*}$ in the final state: $1 / 4$ from spin factors; $J / \psi$ decay 
to electrons: $7 \%$. These factors yield a rate for this reaction of $3.2 \times 10^{-5}$ We have assumed that no appreciable number of higher $K^{*} s$ are produced together with the $J / \psi$.

Aside from the $J / \psi$ region, which has been removed, this study shows various amounts of background falling into the remaining $e^{+} e^{-}$mass distribution.

As a function of various cuts (taken cumulatively) this is:

1. $e^{+} e^{-}$identification: $41 \%$ background or $1.3 \times 10^{-5}$ of $B^{0}$ decays.

2. $K^{0}$ mass cut and $B^{0}$ mass cut: $0.5 \%$ or $1.8 \times 10^{-7}$ of $B^{0}$ decays.

3. A cut on the momentum in the $\Upsilon(4 S) \mathrm{CM}$, for the 4 particles making up the $B$ meson from 0.25 to $0.40 \mathrm{GeV}$. This cut assumes a $5 \mathrm{MeV}$ r.m.s. energy spread.

No background events are observed; if we assume 1 event this corresponds to $0.01 \%$ or $3 \times 10^{-9}$ of $B^{0}$ decays.

We have also studied background from semi-leptonic decay modes of $B^{0}$ and $\mathrm{D}$ in various combinations as well as the case where both $B$ 's decay to a cocktail. No significant background is seen from these modes.

The efficiency for the detection of the chain $B^{0} \rightarrow K^{0} e^{+} e^{-}$ ,$K_{s}^{0} \rightarrow \pi^{+} \pi^{-}$is $1 / 2 \times 2 / 3=1 / 3$. The efficiencies of the three standard cuts are $\epsilon=0.82,0.55$ and 0.52 respectively. This includes the effect of cutting out the $J / \psi$ region. This gives an overall efficiency of $0.33 \times 0.52=0.17$ if we apply all the cuts above.

We have investigated the effect of considering the $e^{+} e^{-}$ mass distribution both as phase space and weighted by a matrix element (due to Bob Cahn) of $\left(M_{B}-2 E_{+}\right)\left(M_{B}-2 E_{-}\right)-$ $M_{K}^{2}$. Here $\mathrm{E}$ is the lepton energy in the $B$ meson CM system. The inclusion of the matrix element shifts the two-lepton mass spectrum to lower masses, but the effect on the detection efficiencies is minimal.

We can, therefore detect the decay mode $B^{0} \rightarrow K^{0} e^{+} e^{-}$ with a $17 \%$ efficiency, giving us a sensitivity to branching ratios of the order of $3.0 \times 10^{-9}$ in our large sample of $B$ mesons. 
Measurement of the $B \rightarrow \tau \nu$ branching fraction is the most viable experimental method of measuring $f_{B}$.
The decay modes $B^{0} \rightarrow K_{0}^{*} e^{+} e^{-}$with decay of the $K_{0}^{*} \rightarrow$ $K^{+} \pi^{-}$and $B^{+} \rightarrow K^{+} e^{+} e^{-}$also have final states with all particles clearly identifiable. They should therefore have comparable detection efficiencies and background problems to the reaction studied here, and be observable at comparable levels of sensitivity.

\section{7. $B^{+} \rightarrow \tau^{+} \nu$ : Determination of $f_{B}$}

The branching fractions for $B^{+}$meson annihilation decays are sensitive to the value of the $B$ decay constant $f_{B}$. Annihilation of $B$ mesons to quarks is, however, difficult to distinguish experimentally from ordinary spectator decays, while purely leptonic decays involving electrons and muons are strongly helicity suppressed. Measurement of the $B^{+} \rightarrow \tau^{+} \nu$ branching fraction is therefore the most viable experimental method of determining $f_{B}$. The rate is given by

$$
\Gamma\left(B_{u} \rightarrow \tau \nu\right)=\left(G^{2} / 8 \pi\right) M_{B} M_{\tau}^{2} f_{B}^{2}\left|V_{u b}\right|^{2}\left(1-M_{\tau}^{2} / M_{B}^{2}\right)^{2}
$$

where $V_{u b}$ is the KM matrix that determines $b \rightarrow u$ transition rates and $f_{B}$ is the decay constant of $B$ mesons. Since $\left|V_{u b}\right|$ can be determined by other processes (e.g. see section 6.5), $B \rightarrow \tau \nu$ can be used to find $f_{B}$. Normalizing to the inclusive semi-leptonic decay rate, we find

$$
B r(B \rightarrow \tau \nu)=5 \times 10^{-5} \times\left(f_{B} / 100 M e V\right)^{2}\left(\left(V_{u b} / V_{c b}\right) / 0.1\right)^{2}
$$

for the branching fraction.

For the purposes of this study we assume a sample of $10^{6}$ charged $B$ decays can be tagged. Section 6.4 shows that such a large number of tags is within the reach of our full data sample. We use the leptonic decays of the $\tau$ (i.e. $\tau \rightarrow l \nu \bar{\nu}$ ) to identify $\tau \nu$ decays of the remaining $B$. Specifically we search for events where in addition to the tracks used in the tag there is a lone lepton and nothing else.

Using the detector and Monte Carlo described in Chapter 3 , we find that in our sample of $10^{6}$ tagged $B$ decays we should find 15 lone lepton events for $f_{B}=100 \mathrm{MeV}$ and $\left|V_{u b} / V_{c b}\right|=$ 0.1 , and 60 lone lepton events for $f_{B}=200 \mathrm{MeV}$. 
Whether a measurement of $f_{B}$ is possible with these events depends, of course, on backgrounds. Potential backgrounds arise from two major sources: a) correctly tagged $B$ 's where all particles from the untagged $B$ other than the lepton are undetected and b) mistagged $B$ 's in which particles from the other $B$ have been mistakenly associated with the tag.

We have studied these potential backgrounds due to correctly tagged $B$ decays by generating Montc Carlo samples in which there is only one $B$ decay. The correct-tag backgrounds would be severe if the detector were not to be designed with this measurement expressly in mind. The Monte Carlo predicts 128 potential background events in our $10^{6}$ tagged event sample. The signal to noise is $\approx 1 / 9$.

All of the potential background events are of the type $B \rightarrow D^{0} l \nu$ where $D^{0} \rightarrow K_{L} \pi^{0}$ or $D^{0} \rightarrow K^{-} l^{+} \nu$. Figure 6.38 shows a scatter plot of $K_{L}$ momentum versus $\cos \theta$ for $K_{L}$ 's from these events. Clearly, the difficulty is caused by the combination of a wide angle $K_{L}$ with a small angle $(|\cos \theta|>0.95)$ $\pi^{0}$. The relatively small number of $K_{L}$ 's at very small angles is due the kinematic bias introduced by seeing the lepton in the detector.

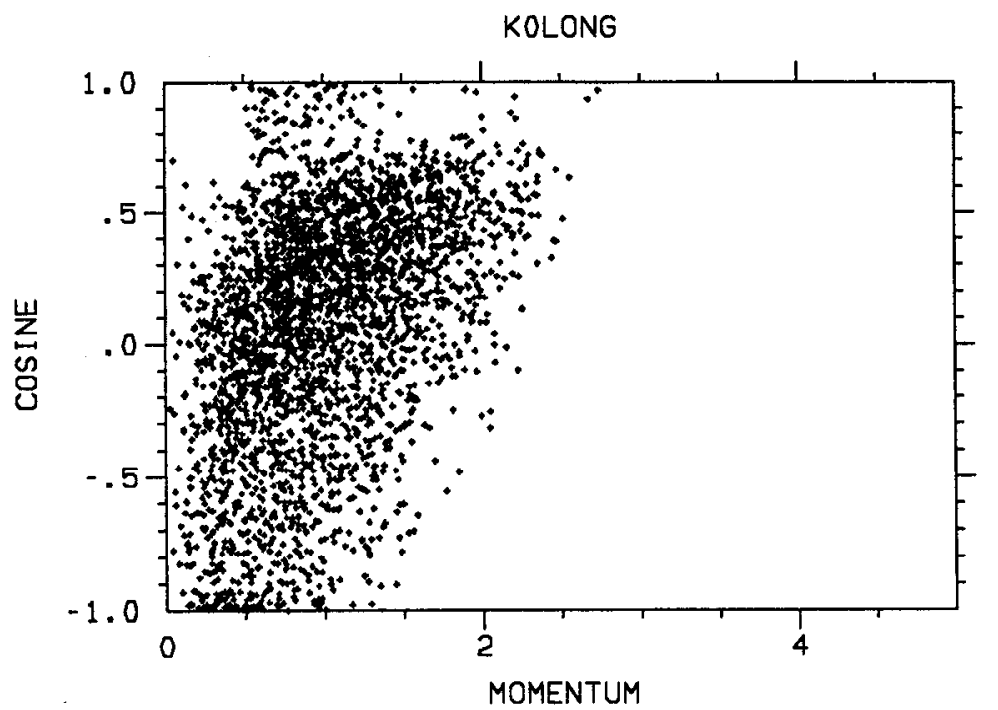

In the case of the $D^{0} \rightarrow K^{-} l^{+} \nu$ source, a high-momentum wide-angle neutrino is associated with low $K l$ cffective mass. Were this low-mass pair to be directed forward, out of the detector acceptance, we would see an apparent lone lepton.
Figure 6.38 Momentum vs. $\cos \theta$ for $K_{L}$ 's from potential background events. 
The nature of the potential background suggests that the detector must include the following elements in order to make a measurement of $B^{+} \rightarrow \tau^{+} \nu_{\tau}$ possible:

- A hadron calorimeter composed of three interaction lengths of thin steel plates sandwiched with scintillator should be able to detect $K_{L}$ 's with momenta greater than about $500 \mathrm{MeV}$ if they interact. In three interaction lengths $\approx 75 \%$ of $K_{L}$ mesons should interact. For purposes of estimating its utility in rejecting background we assume such a calorimeter covers $95 \%$ of the solid angle. However, it is clear from Figure 6.38 that it is not necessary to go that far forward with the calorimeter, and that loss of some forward solid angle could be made up by increasing the number of interaction lengths.

- A small-angle veto system, covering angles down to $8^{\circ}(|\cos \theta|<0.99)$ to reject charged tracks and photons with momenta greater $500 \mathrm{MeV}$ which miss the detcctor proper.

With these features included in the detector, the background is reduced to 1.3 events in $10^{6}$ perfect tags.

In the case of imperfect tags, some of the tracks from the other $B$ decay may be mistakenly associated with the tag, leading to an apparent lone lepton. To investigate this effect we have looked at the tag channel $B^{-} \rightarrow D^{0} l^{-} \nu, D^{0} \rightarrow K^{-} \pi^{+}$ in detail. The tagging efficiency of this channel (based on Monte Carlo) is $0.15 \%$. This leads to a sample of $3.8 \times 10^{5}$ tagged events in the $300 \mathrm{fb}^{-1}$ data sample. Thus the expected signal is $\approx 2$ lone leptons for the nominal values of $f_{B}$ and $V_{u b}$.

CPU time limitations preclude generating enough unbiased Monte Carlo events to investigate these backgrounds. Based on our experience with the perfect tag case, however, we expect that the background will come largely from doublc semi-leptonic decays. By generating only semi-leptonic decays we can increase the statistical power of our sample by a factor of 25 .

In a sample of $5 \times 10^{6}$ double semi-leptonic decays equivalent to $1.2 \times 10^{8}$ unbiased decays we see no background events 
due to imperfect tags. The background we do see is consistent with our expectations for perfect tags. The clean situation in this channel leads us to believe that other tag channels will also prove usable, thereby producing a substantial increase in sensitivity.

We conclude that a measurement of the branching fraction for the $B \rightarrow \tau \nu$ decay mode is possible. Such a measurement requires the detector to be designed with this objective in mind, and, of course, requires a large total integrated luminosity. This fundamental measurement is clearly worth the considerable effort involved. 


\section{REFERENCES}

1. H. Albrecht et al., Phys. Lett. 192B, 245 (1987); ARGUS reports $r=0.21 \pm 0.08$.

2. M. Artuso et al., Phys. Rev. Lett. 62, 2233 (1989); CLEO reports $r=0.19 \pm 0.06 \pm 0.06$.

3. A. J. Buras, W. Slominski \& H. Steger, Nucl. Phys. B245, 369 (1984); G. Altarelli \& P. J. Franzini, Z. Phys. C37, 271 (1988).

4. J. Lee-Franzini, "Results from CUSB at CESR", presented at Les Rencontres de Physique de la Valle d'Aosta, La Thuile, Italy, February 28 - March 5, 1988; PRINT-88-0457 (SUNY at Stony Brook)

5. See, for example, N. Byers and D. S. Hwang, p. 199, Proceedings of the Workshop on High Sensitivity Beauty Physics at Fermilab, ed. A. J. Slaughter, N. Lockyer and M. P. Schmidt, Fermilab, 1987, for other calculations of rates.

6. "Spectroscopy and Decay of Heavy Quarks," by H. Schröder, Proceedings of the XXIV International Conference on High Energy Physics, pp. 73-88; Munich, Fed. Rep. of Germany, August 4-10, 1988; and references therein.

7. P. Krawczyk, D. London and H. Steger, "Prospects for the Measurement of $B_{s} \bar{B}_{s}$ Mixing", DESY, 1988. PRINT-88-0865

8. M. Bauer, B. Stech and M. Wirbel, Z. Phys. C34 103 (1987).

9. A. J. Buras, J. M. Gerard and R. Rueckl, Nucl. Phys. B268, 16, (1986).

10. B. Yu. Blok and M. A. Shifman, Yad. Fiz. 45, 211, (1987).

11. D. Kreinick, Proceedings of the 1989 International Conference on Lepton and Photon Interaction at High Energies, Stanford (1989).

12. K. Schubert, Proceedings of the 1989 Heavy Quark Symposium, Ithaca (1989). 
13. G. Altarelli et al., Nucl. Phys. B208, 365 (1982).

14. B. Grinstein, M.B. Wise and N. Isgur, Phys. Rev. Lett. 56, 298 (1986).

15. N. Isgur, D Scora, B. Grinstein and M.B. Wise, Phys. Rev. D39, 799 (1989).

16. F.J. Gilman and R.L. Singleton, SLAC-PUB-5065 (1989), submitted to Phys. Rev. D.

17. D. Bortoletto, et al., Phys. Rev. Lett. 62, 2436 (1989).

18. M. Wirbel, B. Stech and M. Bauer, Z. Phys. C29, 637 (1985).

19. J.G. Körner and G.A. Schuler, Z. Phys. C38, 511 (1988).

20. J.M. Cline and W.F. Palmer, DESY 89-029 (1989). 


\section{7. $\Upsilon$ PhYSICS AT AN ASYMMETRIC $B$ FACTORY}

\subsection{INTRODUCTION}

$\mathrm{Q}$

uarkonia, or bound states of quark and antiquark, provide us with an excellent testing ground for quantum chromodynamics (QCD), both perturbative and non-perturbative. Of the known quarkonia systems, bottomonium $(b \bar{b})$ is the heaviest known to date. It is the most amenable to theoretical interpretation because both relativistic effects and higher order perturbative QCD corrections are less important than in the lighter quark systems. The $\Upsilon$ system has a very rich spectrum, as can be seen in Figure 7.1, and although many of the expected states have been observed, there still remain a large number of important spectroscopic measurements that must be made. However, much of this physics is only accessible with large data samples of a few tens of millions of $\Upsilon$ resonance events.

We believe that the rich physics potential of the $\Upsilon$ system will remain largcly untapped after 1990 , until such time as a high luminosity $B$ factory is available, together with a very high quality electromagnetic calorimeter. Thus, in this report, we discuss the unique $\Upsilon$ physics opportunities available at an asymmetric $B$ factory. The physics topics include:

1. the possible discovery of the pseudoscalar and pseudovector states and the measurement of the hyperfine structure of the $b \bar{b}$ system from the measurement of transitions from the $\Upsilon(3 S)$ and $\Upsilon(2 S)$ states to the singlet $S$ states, $\Upsilon\left(n^{1} S_{0}\right)$, otherwise known as the $\eta_{b}$ states, and to the lowest singlet $P$ state, $\Upsilon\left(1^{1} P_{1}\right)$, the $h_{b}$

2. the discovery and measurement of the $\Upsilon(1 D)$ states via radiative transitions,

3. detailed studies of the known triplet $P$ states (the $\chi_{b}$ 's),

4. detailed studies of hadronic transitions between $\Upsilon$ states,

5. high statistics studies of radiative $\Upsilon$ decays, inclüing searches for light neutral Higgs,

6. high precision physics measurements such as a comparison of the $B_{\mu \mu}$ to $B_{\tau \tau}$ rate, which is sensitive to the 
compositeness scale, the $\chi_{b}$ and $\chi_{b}^{\prime}$ fine structure, where tagged events can be used in gluon vs quark fragmentation studies, E1 rates, which can be used for potential model relativistic correction studies, etc.

The richness of $\Upsilon$ physics has not gone unnoticed in other $B$ factory studies. 1,2

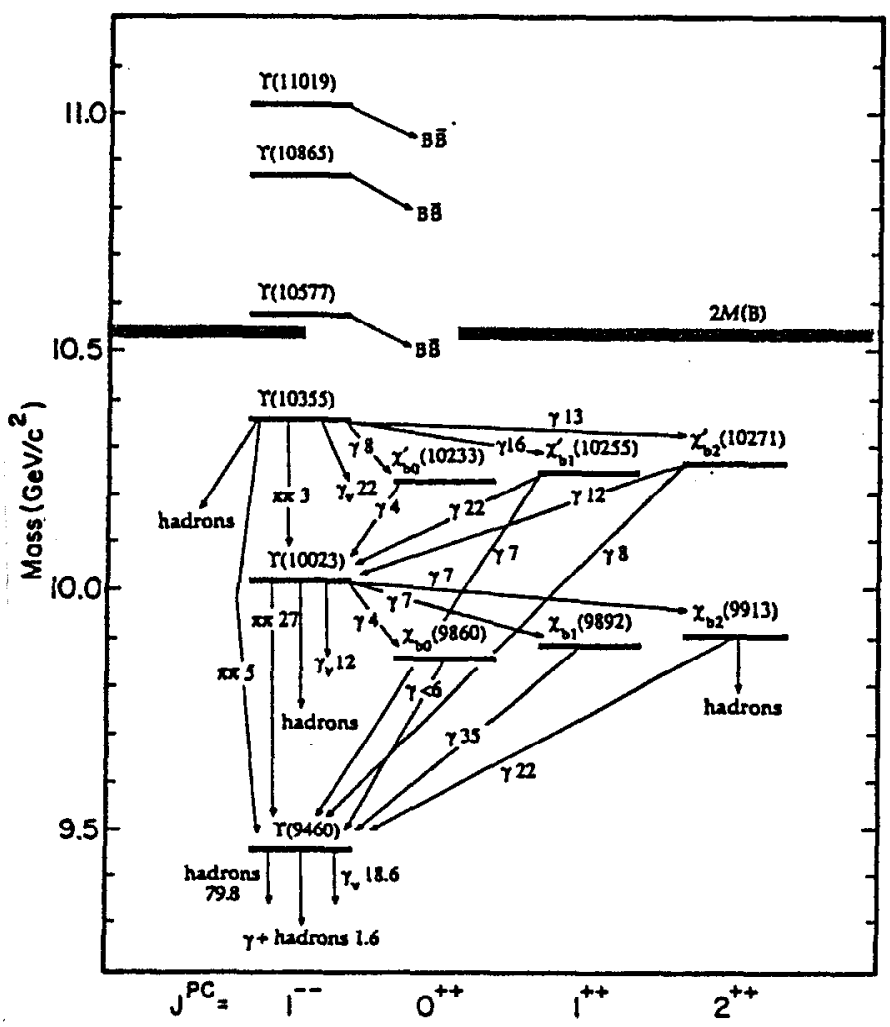

The possibility of performing precision spectroscopic measurements is strongly dependent on electromagnetic calorimetry with superior resolution. We discuss, in the following, the quality of calorimetry needed to carry out $b \bar{b}$ spectroscopy studies. In particular we will focus on the requirements for (1) the angular resolution, (2) the intrinsic energy resolution, and (3) the low energy photon cutoff. We should also note that these high luminosities combined with the relatively large $\Upsilon(1 S)$ hadronic cross section will lead to the necessity of handling data rates as high as $100 \mathrm{~Hz}$.
The only detectors running in the $\Upsilon$ region in the early 1990's will be CLEO and ARGUS. Neither will have the integrated luminosity or detector resolution to carry out these precision spectroscopic studies

Figure 7.1 The $\Upsilon$ spectrum. 


\subsection{A Review of the Present Status}

There are numerous reviews of quarkonia ${ }^{3-6}$ available which summarize the present situation. In this section, we will only discuss some of the topics which are relevant to the $\Upsilon$ physics that will be studied at a $B$ factory.

Data Samples The potentially vast data samples available at a "modest" $B$ factory offer the unique capability of probing branching ratios on the order of $10^{-5}$, which is the level at which much of the remaining physics lies. In Table 7.1, we have tabulated the total (integrated over all the experiments: CUSB, CLEO, ARGUS and Crystal Ball) present data samples available on the $\Upsilon$ resonances, where we have included a large $\left(\approx 1.5 \times 10^{6}\right)$ sample of $\Upsilon(3 S)$ events that will be obtained by CUSB this final running period at CESR. The present samples are compared in the Table with the data samples one could obtain from a $B$ factory running at $\mathcal{L}_{\text {peak }} \approx 10^{33} \mathrm{~cm}^{-2} \mathrm{sec}^{-1}$ with a beam spread $\sigma=5 \mathrm{MeV}$ (to be compared with the CESR width of $\approx 3.5 \mathrm{MeV}$ ). We have assumed that the accelerator is capable of delivering 50 $\mathrm{pb}^{-1} /$ day.

Table 7.1 Comparison of present data samples with 3 month runs at $\mathcal{L}_{\text {peak }} \approx 10^{33} \mathrm{~cm}^{-2} \mathrm{sec}^{-1}$.

\begin{tabular}{|l|c|c|}
\hline & 1990 Sample & 3 months $B$ Factory \\
\hline$\Upsilon(1 S)$ & $1.5 \times 10^{6}$ & $70 \times 10^{6}$ \\
\hline$\Upsilon(2 S)$ & $0.5 \times 10^{6}$ & $25 \times 10^{6}$ \\
\hline$\Upsilon(3 S)$ & $3.0 \times 10^{6}$ & $15 \times 10^{6}$ \\
\hline
\end{tabular}

As Table 7.1 illustrates, there is an opportunity to obtain data samples in a single detector which are 5 to 50 times larger than all accumulated future data samples at CESR and DORIS. The type of $\Upsilon$ physics described below, will remain untouched until these kind of data samples are accumulated. It is also important to note that these samples were calculated using very conservative assumptions; three months is a relatively short run, the luminosity we have assumed is below that which the storage rings will reach at an early stage, and 
the beam energy spread may be smaller than the $5 \mathrm{MeV}$ we assumed.

$\alpha_{s}$ Determinations The $\Upsilon$ system can supply some of the most precise values of $\alpha_{s}$ (subject, however, to varying degrees of theoretical uncertainty). These values are determined from measurements of $B_{\mu \mu}$ and $B_{g g \gamma}$. For example, using the most recent values $^{7-9}$ of $B_{\mu \mu}$ shown in Table 7.2, we presently find the best values of $\alpha_{s}$ and consequently $\Lambda_{\overline{M S}}$ to be those shown in Table 7.3.

Table 7.2 Recent experimental values for $B_{\mu \mu}$.

\begin{tabular}{|c|c|c|c|}
\hline & $B_{\mu \mu}(1 S)(\%)$ & $B_{\mu \mu}(2 S)(\%)$ & $B_{\mu \mu}(3 S)(\%)$ \\
\hline CUSB & $2.61 \pm .09 \pm .1$ & $1.38 \pm .25 \pm .2$ & $1.73 \pm .15 \pm .1$ \\
\hline CLEO & $2.52 \pm .07 \pm .1$ & & $2.02 \pm .19 \pm .3$ \\
\hline ARGUS & $2.30 \pm .25 \pm .1$ & & \\
\hline Average & $2.53 \pm 0.08$ & $1.38 \pm 0.29$ & $1.79 \pm 0.17$ \\
\hline
\end{tabular}

Table 7.3 Computed quantities from $B_{\mu \mu}$.

\begin{tabular}{|c|c|c|c|c|}
\hline & $\Gamma_{\text {tot }}(\mathrm{keV})$ & $\Gamma_{g g g} / \Gamma_{\mu \mu}$ & $\alpha_{s}(4.9 \mathrm{GeV})$ & $\Lambda_{\overline{\mathrm{MS}}}(\mathrm{MeV})$ \\
\hline$\Upsilon(1 S)$ & $53 \pm 2.6$ & $32.1 \pm 1.5$ & $.176 \pm .003$ & $164 \pm 10$ \\
\hline$\Upsilon(2 S)$ & $43 \pm 9.1$ & $32.5 \pm 8.3$ & $.176 \pm .015$ & $167 \pm 54$ \\
\hline$\Upsilon(3 S)$ & $25 \pm 2.8$ & $29.3 \pm 3.6$ & $.171 \pm .007$ & $146 \pm 24$ \\
\hline
\end{tabular}

While the statistical errors are generally small, there still is room for improvement for the $\Upsilon(2 S)$ measurement, which has an error of $\approx 10 \%$. The theoretical uncertainties are significantly larger; it is an area in which we could hope for improvement in the future.

Interpretation of $B_{g g \gamma}$ might be on a more secure theoretical footing, but there is considerable need for improved experimental measurements. 
Figure 7.2 Observed exclusive photon spectrum.

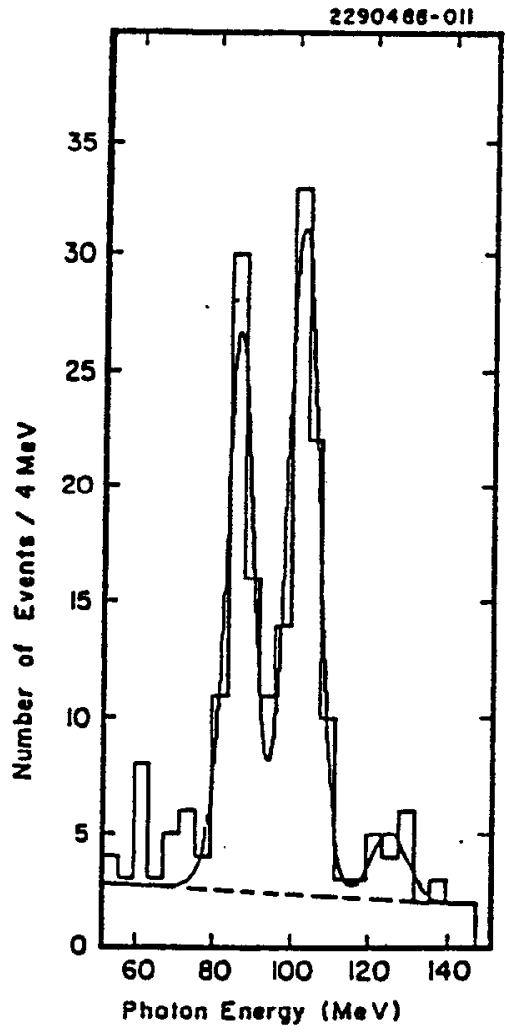

Spectroscopic measurements

The fine structure of the $\Upsilon$ system is presently quite well measured (e.g., Table 7.4 shows the CUSB results from the exclusive channels shown in Figure 7.2), except for some of the smaller branching ratio transitions.

Table 7.4 Measured fine structure for $\Upsilon(2 P)$ from $3 S \rightarrow \gamma 2 P_{J} \rightarrow \gamma \gamma(1 S$ or $2 S) \rightarrow \gamma \gamma \ell^{+} \ell^{-}$.

\begin{tabular}{|c|c|c|}
\hline$\Upsilon\left(2^{3} P_{J}\right)$ & $E_{\gamma}(\mathrm{MeV})$ & Events \\
\hline $\mathrm{J}=2$ & $86.1 \pm 0.5$ & $104.9 \pm 11.7$ \\
\hline $\mathrm{J}=1$ & $101.5 \pm 0.6$ & $122.5 \pm 12.5$ \\
\hline $\mathrm{J}=0$ & $122.4 \pm 2.6$ & $12.3 \pm 5.1$ \\
\hline
\end{tabular}

The measured fine structure has imposed constraints on model builders since the observed fine structure can be compared to the generalized formulation of the spin dependence of the $Q \bar{Q}$ potential. ${ }^{10}$ By inserting potentials of known Lorentz transformation properties in the Bethe-Salpeter equation and making a nonrelativistic reduction to order $v^{2} / c^{2}$, the fine structure of the $\chi_{b}$ states can be related to a vector potential, 
$V$, and a scalar potential, $S$ by:

$$
\begin{aligned}
& M\left({ }^{3} P_{2}\right)=\bar{M}+a-2 b / 5, \\
& M\left({ }^{3} P_{1}\right)=\bar{M}-a+2 b, \\
& \text { and } M\left({ }^{3} P_{0}\right)=\bar{M}-2 a-4 b \\
& \text { where } a=\frac{1}{2 M_{Q}^{2}}\left\langle 3 V^{\prime} / r-S / r\right\rangle \\
& \text { and } b=\frac{1}{12 M_{Q}^{2}}\left\langle V^{\prime} / r-V^{\prime \prime}\right\rangle
\end{aligned}
$$

are the spin-orbit and tensor contributions respectively, and $\bar{M}$ is the center-of-gravity of the triplet $\mathrm{P}$ states. ${ }^{11}$ The principal difference between potential models (e.g., $\mathrm{MR}^{12} \mathrm{MB}^{13}$ and $\left.\mathrm{GRR}^{14}\right)$ is their choice of $V$ and $S$. Agreement of the data with the GRR model is usually taken as support for a long range confining potential that transforms as a scalar. However, the situation may indeed be more complicated (see the discussion in section 7.3).

The electric dipole transition rates for $\Upsilon(3 S) \rightarrow \gamma \chi_{b}^{\prime}$ are quite well measured, however the $\Upsilon(3 S) \rightarrow \gamma \chi_{b} \rightarrow \gamma \gamma \Upsilon(1 S)$ transition has only just been seen by CUSB and is very poorly measured at present $\left(B R\left(\Upsilon(3 S) \rightarrow \chi_{b} \gamma\right) \times B R\left(\chi_{b} \rightarrow \Upsilon(1 S) \gamma \approx\right.\right.$ $\left.(9 \pm 5) \times 10^{-4}\right)$. The interest in this product branching ratio lies in the fact that it is particularly sensitive to relativistic corrections.

\section{Hadronic Transitions}

The $\pi \pi$ hadronic transition rates between $\Upsilon$ states have to date been successfully described in terms of a QCD multipole expansion of the gluon fields. ${ }^{15} \mathrm{CLEO}^{16}$ has now obtained a large sample of $\Upsilon(3 S) \pi \pi$ hadronic decays in which the observed dipion invariant mass spectrum differs from that predicted by ref. 15 (where one uses the soft pion theorems of PCAC), and is not presently well understood theoretically. Higher statistics for the transitions $\Upsilon(3 S) \rightarrow \pi \pi \Upsilon(2 S)$ would provide useful information in order to clarify the situation. 


\section{Radiative $\Upsilon$ Decays and Searches for New Particles}

High statistics studies of radiative $\Upsilon$ decays provide us with an excellent laboratory in which to search for rare processes. At present, only the inclusive rate for $\Upsilon \rightarrow \gamma g g \rightarrow$ $\gamma+$ hadrons has been measured (see Table 7.5).

Table 7.5 Latest $B R(\Upsilon \rightarrow \gamma g g)$ values .

\begin{tabular}{|l|c|c|c|}
\hline & CUSB & CLEO & ARGUS \\
\hline$B R(\Upsilon \rightarrow \gamma g g)(\%)$ & $3 \pm 0.6$ & $2.5 \pm 0.2$ & $3 \pm 0.3$ \\
\hline
\end{tabular}

This same process has also been used to determine $\alpha_{s}$. However the branching ratio values are obtained by integrating over the high end of the direct photon spectrum $\left(E_{\gamma} / E_{\text {beam }}>\right.$ $0.5)$, and thus still subject to uncertainties.

Exclusive radiative processes, $\Upsilon \rightarrow \gamma+$ light mesons, have not yet been seen, but experiment is approaching the level of sensitivity at which one expects to observe them, for instance from simple scaling from the $c \bar{c}$ system, as shown in Table 7.6.

Table 7.6 $90 \%$ CL UL for $\Upsilon \rightarrow \gamma+$ light meson.

Both BR's and the scaled values are shown $\times 10^{4}$.

\begin{tabular}{|c|c|c|}
\hline Final State & $B R_{\text {expt }}$ & Prediction \\
\hline$\gamma \eta(550)$ & $<3.5(\mathrm{CB})$ & 0.2 \\
\hline$\gamma \eta^{\prime}(957)$ & $<13(\mathrm{CB})$ & 1 \\
\hline$\gamma f_{2}(1270)$ & $<8.1(\mathrm{CB})$ & 0.3 \\
\hline$\gamma f_{2}(1270), f_{2} \rightarrow \pi^{ \pm} \pi^{\mp}$ & $<1.2(\mathrm{CLEO})$ & \\
\hline$\gamma f_{2}(1720), f_{2} \rightarrow K_{S}^{0} K_{S}^{0}$ & $<3.6(\mathrm{CLEO})$ & \\
\hline$\gamma f_{2}(1720), f_{2} \rightarrow \eta \eta$ & $<4.3(\mathrm{CB})$ & \\
\hline$\gamma f_{2}(1720), f_{2} \rightarrow K^{ \pm} K^{\mp}$ & $<1.2(\mathrm{CLEO})$ & 0.1 \\
\hline$\gamma \eta(1430), \eta \rightarrow K^{ \pm} \pi^{\mp} K_{S}^{0}$ & $0.82(\mathrm{CLEO})$ & 1 \\
\hline
\end{tabular}

Finally, $\Upsilon$ radiative decays are an excellent laboratory in 
which to search for the elusive Higgs. The present CUSB limits on monochromatic searches in the process $\Upsilon \rightarrow \gamma+X$ are at the level of $10^{-4}$. At this level of sensitivity we are probing the Standard Model predictions (including radiative corrections) for the decay $\Upsilon \rightarrow \gamma+$ Higgs. The present search from CUSB rules out Higgs of masses up to $\approx 5 \mathrm{GeV}$, although it is clear that more theoretical work needs to be done in calculating the radiative corrections.

\section{3. $\Upsilon$ Physics Capabilities at High Luminosities}

Having seen the rich physics potential of the $\Upsilon$ system, let us now turn to look in some detail at those topics which offer unique physics opportunities at a $B$ factory.

Measuring the Hyperfine Structure

One of the open questions in quarkonia is the nature of the spin-spin interaction (responsible for the hyperfine structure) which is very short ranged and presumably mediated predominantly by single gluon exchange. The singlet states of heavy quarkonia have always been difficult to find relative to their triplet cousins. They are not produced directly in $e^{+} e^{-}$annihilations, and thus at $e^{+} e^{-}$colliders they are usually searched for by detecting monochromatic photons resulting from magnetic dipole transitions between higher triplet $\mathrm{S}$ wave states and the lower lying singlet $S$ wave states, $n^{1} S_{0}$ or $\eta_{b}$ 's. The rates involved are considerably suppressed relative to the electric dipole transition rates. In the upsilon system the largest rate is expected for the $\Upsilon(1 S)$ ground state decay to the lowest lying $\eta_{b}$, where

$$
B R\left(\Upsilon\left(n^{3} S_{1}\right) \rightarrow \gamma \Upsilon\left(n^{1} S_{0}\right) \approx 10^{-3}\left(E_{\gamma}(M e V) / 100\right)^{3}\right.
$$

At present, using $\approx 4 \times 10^{5} \Upsilon$ 's, CUSB places a upper limit for the $\operatorname{BR}\left(\Upsilon(1 S) \rightarrow \gamma \eta_{b}\right)$ of $<0.005-0.007$ for $30 \mathrm{MeV}<$ $E_{\gamma}<100 \mathrm{MeV}$ at the $90 \%$ confidence level. ${ }^{17}$ This agrees well with the expected scaling of M1 transitions with the magnetic moment, $\propto\left|e_{Q} / \dot{M}_{Q}\right|^{2} \approx 0.01$ when compared with $\mathrm{BR}\left(\mathrm{J} / \psi \rightarrow \gamma \eta_{\mathrm{c}}\right) \approx 0.013$. With $\approx 7 \times 10^{7} \Upsilon$ events, one can conservatively expect a factor of 10 improvement in the upper limits (we are ignoring improved $\pi^{0}$ reconstruction efficiency 
which will significantly reduce backgrounds), or perhaps one will be able to observe the $\Upsilon\left(1^{1} S_{0}\right)$ if the hyperfine splitting is sufficiently large.

One can also search for monochromatic photons from the hindered M1 transitions, $\Upsilon(3 S) \rightarrow \gamma \eta_{b}^{\prime}$ and $\Upsilon(2 S) \rightarrow \gamma \eta_{b}$. The predictions for these branching ratios are sensitive to relativistic corrections, but they lie $\mathrm{e}^{18}$ in the range of $10^{-4}$ to $10^{-6}$. These will be extremely hard to observe if they exist at the smallest branching ratios, since they must be observed in the inclusive photon spectrum.

Kuang and Yan have suggested a search for the singlet states by studying the decay chain $\Upsilon(3 S) \rightarrow \pi \pi^{1} P_{1} \rightarrow \pi \pi \gamma \eta_{b}$, because the branching ratio of the hadronic transition is expected to be about $1 \%$, and that of the following E1 transition about $50 \%$, yielding a product branching ratio for the wholc chain of about $5 \times 10^{-3}$, the largest branching ratio by far of all channels leading to singlet states. ${ }^{19}$ Unfortunately, the $\eta_{b}$ does not decay leptonically, therefore we cannot use the "exclusive" event method. We can, however, search for a monochromatic photon line of about $480 \mathrm{MeV}$ in the inclusive photon spectrum, the result of a transition from the singlet $P$ wave to singlet $S$ wave, in events with two pions recoiling against a state of mass near the center-of-gravity of the $\chi_{b}$ states. This process has the added bonus that one observes not only the singlet $S$ states but also the singlet $P$ state (which still remains undiscovered in charmonium as well as bottomonium). In addition, the dual requirement of observing both the $\pi \pi$ transition and the $\approx 480 \mathrm{MeV}$ photon will effectively eliminate the possible backgrounds from the competing and more copious E1 transitions via the $\chi_{b}$ states. If the Kuang, Yan and Tuan branching ratio predictions ${ }^{20}$ are correct, the singlet $S$ state may be seen by CUSB in the upcoming CESR run, however, there is the possibility raised by Voloshin, ${ }^{21}$ who uses a different treatment of the soft gluon conversion to hadrons, that this $\pi \pi$ transition is greatly suppressed, with $B R\left(\Upsilon(3 S) \rightarrow \pi \pi h_{b}\right)<10^{-4}$. If this is the case, then this transition will remain undetected until large (few $\times 10^{7}$ events) are available. In even the most optimistic scenarios, the higher singlet $S$ states, $\eta_{b}^{\prime}$ and $\eta_{b}^{\prime \prime}$, will remain 
undetected until a high luminosity $B$ factory is built.

Another promising method to detect the singlet $S$ state is via the decay $\Upsilon \rightarrow \gamma \Upsilon\left(2^{3} P_{1}\right) \rightarrow \gamma \pi \pi \Upsilon\left(1^{1} S_{0}\right)$ where the branching ratio may be as high as $10^{-3}$ but the process will remain undetected until a detector with both excellent photon detection and excellent charged particle tracking is built (present detectors do not manage to optimize both at once).

In summary, the prospects for observing and measuring the singlet $S$ state in the $\Upsilon$ system look very promising.

D-wave State Spectroscopy

Recently, Kwong and Rosner have used the "inverse scattering" method to construct ${ }^{22}$ the $b \bar{b}$ potential and in turn determine additional $b \bar{b}$ states. They find two bound D wave states which lie below the free flavor threshold and have their centers of gravity (cog's) at $\approx 10.16(1 \mathrm{D})$ and $10.44(2 \mathrm{D}) \mathrm{GeV}$. They also find the fine structure splittings to be very small, of the order of 4-6 MeV instead of the $15-20 \mathrm{MeV}$ characteristic of the $\chi_{b}^{\prime}$ fine structure. These predictions are supposed to be largely model independent, not only because the "inverse scattering" method is based on using measured $S$-wave and $P$-wave levels, but, as A. Martin shows, they also follow from general mathematical properties common to most nonrelativistic potentials. ${ }^{23}$ These states, however, are not easy to observe, their leptonic widths are minute, and lying so far below threshold, one does not expect much S-D mixing enhancement. At $e^{+} e^{-}$colliders, therefore, we can only access these states via two sequential $\mathrm{E} 1$ transitions, i.e. from $\Upsilon(3 S) \rightarrow \gamma \chi_{b}^{\prime} \rightarrow \gamma \gamma \Upsilon_{J}(1 D)$, with product BR's of the order of $3.7(3.2) \times 10^{-3}$ for the $J=2(3)$ final states. Recent calculations by Belanger and Moxhay have shown that for $\mathrm{D}$-wave quarkonia, the three-gluon annihilation rate is very small. ${ }^{24}$ Moxhay has shown that the hadronic transition rates are also very small, thus the $\mathrm{D}$ states essentially all decay electromagnetically to the $\chi_{b}$ states.

Since these predictions are firm and essentially modelindependent, the observation (or nonobservation) of such states at the predicted masses constitutes a real test of the validity of the current potential model description of heavy quarkonia. 
Figure 7.3 Photon spectrum from $\Upsilon(3 S) \rightarrow \gamma \gamma \gamma \gamma \ell^{+} \ell^{-}$ decays.
The region of the potential probed by heavy quarkonia spectra lies neither in the perturbative regime nor in the relativistic one. There are valid arguments that gluon exchange within quarkonia systems might be more similar to gluon propagation through the gluon condensate, which means they cannot be described ${ }^{26}$ using non relativistic potentials.

Thus, two ways present themselves for the search of $D$ wave states. The first is to look in the inclusive spectrum for monochromatic photon lines characteristic of $\chi_{b}^{\prime} \rightarrow \Upsilon_{2,3}(1 D) \gamma$ transitions, with $\mathrm{E}_{\gamma} \approx 100(110 \mathrm{MeV})$ respectively, and of $\Upsilon_{3,2}(1 D) \rightarrow \chi_{b} \gamma$ transitions, with $\mathrm{E}_{\gamma} \approx 230(240 \mathrm{MeV})$. Unfortunately, all but the $260 \mathrm{MeV}$ line are going to be masked by the much stronger $\left(\Upsilon(2 S) \rightarrow \chi_{b}^{\prime} \rightarrow \Upsilon(2 S)\right)$ photon lines. Such a measurement would be extremely difficult.

Given the large amount of data available at a $B$ factory, we believe that

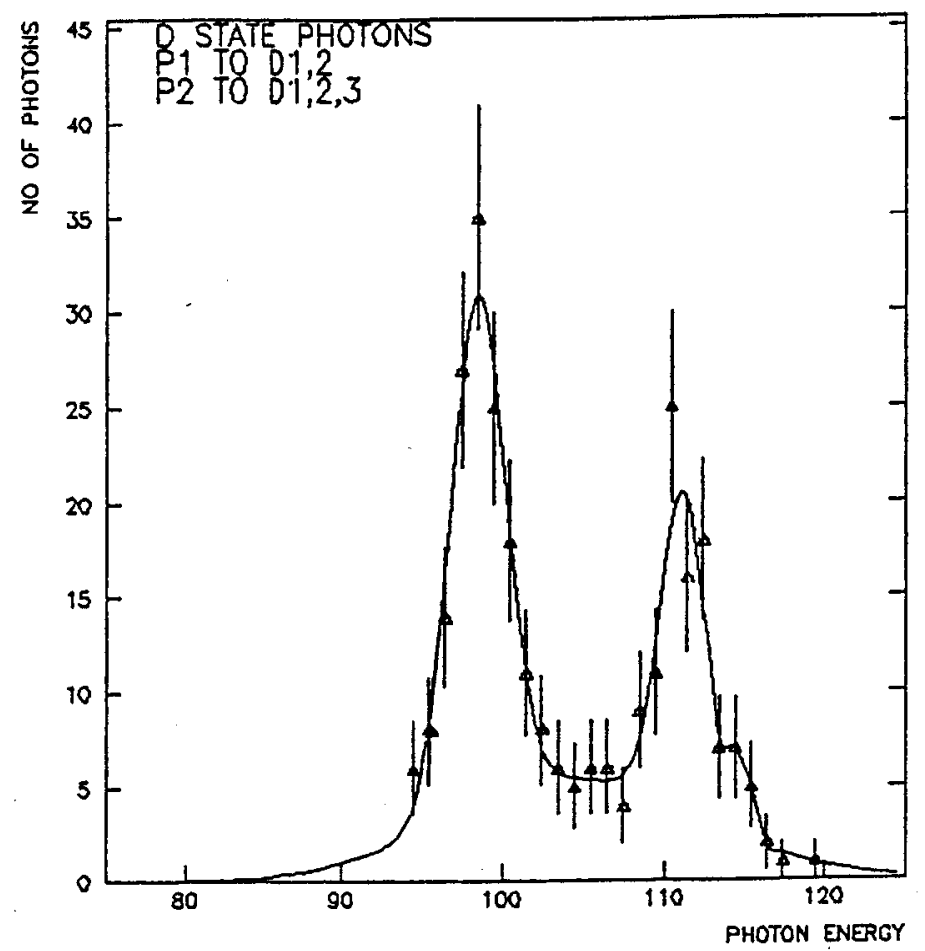

one can afford to give up some on statistics and concentrate on the lower branching ratio but cleaner sample of multiphoton exclusive events. This means searching for the $D$ wave states by capitalizing on the relatively large branching ratio 
for the transition $\chi_{b} \rightarrow \Upsilon(1 S) \gamma$ and that the $\Upsilon(1 S)$ decays leptonically some $3 \%$ of the time. Hence the product BR for $\Upsilon(3 S) \rightarrow \chi_{b}^{\prime} \gamma \rightarrow \Upsilon_{2}(1 D) \gamma \gamma \rightarrow \chi_{b} \gamma \gamma \gamma \rightarrow \Upsilon(1 S) \gamma \gamma \gamma \gamma \rightarrow$ $\mu \mu \gamma \gamma \gamma \gamma$ is $\approx 3 \times 10^{-5}$ and for $\Upsilon(3 S) \rightarrow \chi_{b}^{\prime} \gamma \rightarrow \Upsilon_{3}(1 D) \gamma \gamma \rightarrow$ $\chi_{b} \gamma \gamma \gamma \rightarrow \Upsilon(1 S) \gamma \gamma \gamma \gamma \rightarrow \mu \mu \gamma \gamma \gamma \gamma$ is $\approx 2 \times 10^{-5}$. While these product $\mathrm{BR}$ 's are small, the events would be very, very clean: four photons of the appropriate energies accompanied by two leptons. A small complication does arise from the fact that the level splittings conspire to make the $\Upsilon(3 S) \rightarrow$ $\chi_{b}^{\prime} \gamma \rightarrow \Upsilon_{2}(1 D) \gamma \gamma \rightarrow \chi_{b} \gamma \gamma \gamma \rightarrow \Upsilon(1 S) \gamma \gamma \gamma \gamma$ transition indistinguishable from the $\Upsilon(3 S) \rightarrow \chi_{b}^{\prime} \gamma \rightarrow \Upsilon(2 S) \gamma \gamma \rightarrow \chi_{b} \gamma \gamma \gamma \rightarrow$ $\Upsilon(1 S) \gamma \gamma \gamma \gamma$ transition, although the latter process has the smaller product $\mathrm{BR}$ of $\approx 1 \times 10^{-5}$. However, with the necessary angular resolution, one can perform kinematic fits of the four photons and two leptons, which will effectively eliminate any confusion.

We have carried out a Monte Carlo study for $D$ state exclusive spectroscopy of the type outlined above. The expected observed spectrum from a run corresponding to $1.5 \times$ $10^{7} \Upsilon(3 S)$ decays is shown in Figure 7.3 , where we have assumed an angular resolution for the detector of $10 \mathrm{mr}$, and a photon energy resolution of $\sigma_{E} / E=1 \% / \sqrt[4]{E}$, where $E$ is the photon energy in $\mathrm{GeV}$, and a very modest photon finding efficiency of $50 \%$. The observed spectrum consists of photons from the decays shown in Table 7.7.

Table 7.7 Assumed D state branching ratios. Only dominant ones shown.

\begin{tabular}{|c|c|c|}
\hline Decay & $E_{\gamma}(\mathrm{MeV})$ & $B R$ \\
\hline $3 S \rightarrow 2 P_{1} \rightarrow 1 D_{2} \rightarrow 1 P \rightarrow 1 S$ & 98.6 & $2 \times 10^{-5}$ \\
\hline $3 S \rightarrow 2 P_{1} \rightarrow 1 D_{1} \rightarrow 1 P \rightarrow 1 S$ & 104.6 & $3 \times 10^{-6}$ \\
\hline $3 S \rightarrow 2 P_{2} \rightarrow 1 D_{3} \rightarrow 1 P \rightarrow 1 S$ & 110.6 & $1 \times 10^{-5}$ \\
\hline $3 S \rightarrow 2 P_{2} \rightarrow 1 D_{1} \rightarrow 1 P \rightarrow 1 S$ & 114 & $3 \times 10^{-6}$ \\
\hline
\end{tabular}

Notice that even with the excellent resolution, is still not possible to fully resolve the $\mathrm{D}$ state lines because of their minute expected splittings, but it will still be possible to extract fine structure information by fitting the above observed
$D$ state spectroscopy imposes the strictest requirements on the detector calorimetry, the response can be nothing short of superb. 
Figure 7.4 Photon spectrum from $\Upsilon(3 S) \rightarrow \gamma \gamma \gamma \gamma \ell^{+} \ell^{-}$ decays for $\sigma_{E} / E=2 \% / \sqrt[4]{E}$. spectrum to the sum of four lines coming from $\Upsilon\left(2^{3} P_{1,2}\right)$ decays to the 1D state. In particular, we have found that we are able to measure some D state splittings to a few \%. For comparison, we have also considered what the spectrum would look like if the resolution were $\sigma_{E} / E=2 \% / \sqrt[4]{E}$. The result is shown in Figure 7.4.

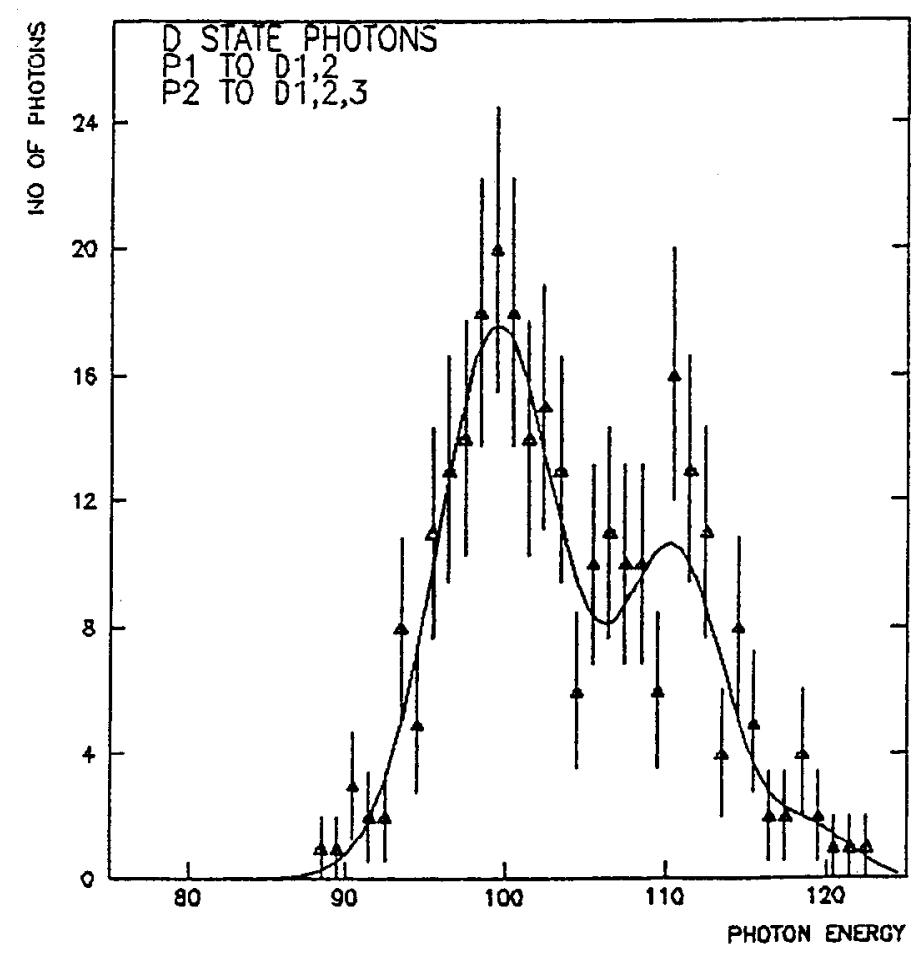

$\underline{\chi_{b} \text { and } \chi_{b}^{\prime} \text { Studies }}$

As indicated in the earlier summary of known $\Upsilon$ physics, the fine structure of the $\chi_{b}$ and $\chi_{b}^{\prime}$ states is quite well known, but there still remains some physics that is only accessible at the highest luminosities.

\section{Relativistic Corrections}

The measurement of the $\Upsilon(3 S) \rightarrow \chi_{b}$ transition is of interest since it bears on the relativistic corrections to be applied in potential model construction. With the b-quark being quite heavy, relativistic effects do not manifest themselves as readily as in the lighter quark systems. However, when they do, it seems to be relatively easy to pinpoint the source, and study them in relative isolation. A good 
measurement of the $\Upsilon(3 S) \rightarrow \gamma \chi_{b}$ rate, in particular, will serve to constrain the varieties of potential modcls on the market which are indistinguishable as far as static properties and predictions are concerned. We also have further interest in knowing this rate because of the possible overlap between this signal and that of the $h_{b} \rightarrow \gamma \eta_{b}$ transition in the inclusive photon spectrum from $\Upsilon(3 S)$ decays. With $\approx 1.5 \times 10^{7} \Upsilon(3 S)$ events, and assuming the CUSB value of $B R\left(\Upsilon(3 S) \rightarrow \gamma \chi_{b} \rightarrow \gamma \gamma \Upsilon(1 S) \rightarrow \gamma \gamma \mu^{+} \mu^{-}\right) \approx 3 \times 10^{-5}$, we should be able to collect a sample of a few hundred such events.

\section{The Nature of the Spin-Dependent Potential}

From the known measurements of the $P$ state fine structure, it is usually inferred that the interquark potential is made up of short range vector contribution and a long range scalar confining contribution. This conclusion is usually reached because of the agreement of the observed fine structure with potential models that assume a long range scalar confining term, such as the Gupta, et al. model. However other models also assume a similar long range scalar confining term but disagree with the data. A closer inspection of the fine structure reveals an apparent fallacy in the simple picture presented with respect to the Lorentz structure of potentials $V$ and $S .^{27}$ Consider $r$ in terms of $a$ and $b$ (defined in an earlier section) for P-wave states, $r=[2 a-(12 / 5) b] /[a+6 b]$. For a Coulombic potential $(S=0, V \propto-1 / R), r=0.8$. Note also that if $S$ is not $0, a$ decreases and $r$ decreases. Thus, since the rms radius of the $\chi_{b}^{\prime}$ is greater than that of the $\chi_{b}$, it should feel the confining "scalar" potential more strongly, hence $r\left(\chi_{b}^{\prime}\right)$ should be less than $r\left(\chi_{b}\right)$. Yet neither the data nor explicit potential model calculations support this simple deduction. Dib, Gilman and Franzini (DGF), with numerical computations, resolve this apparent contradiction. ${ }^{28}$ They conclude that one cannot make a simple assignment of the Lorentz structure (that it is the fourth component of a Lorentz vector or a Lorentz scalar) to pieces of a potential and expect it to hold true over the whole range of the interactions. A. Martin proved and extended the DGF conjectures and in addition, showed that for the standard potentials used to describe 
heavy quarkonium, the overall splitting of the higher $\mathrm{P}$ states is likely to be smaller than the lower $\mathrm{P}$ states. ${ }^{29}$ This last statement is indeed borne out in the $b \bar{b} \mathrm{P}$ states: the overall splitting for $\chi_{b}^{\prime}$ is $34.6 \mathrm{MeV}$ and for the $\chi_{b}$ is $53.5 \mathrm{MeV}$.

Higher precision results on the fine structure of both the $\chi_{b}$ and the $\chi_{b}^{\prime}$ should provide valuable experimental input to this question.

\section{Hadronic Widths of the $\Upsilon\left(n^{3} P_{J}\right)$ States}

Unlike in the charmonium system, the hadronic widths of the $\chi_{b}$ states are too narrow to be measured directly. We recall that CUSB had pioneered the method of obtaining them from the measured branching ratios for ${ }^{3} P_{J} \rightarrow{ }^{3} S_{1}+\gamma$ transitions $\left(B R_{J}\right)$ using the $\mathrm{E} 1$ rates calculated from potential models, see section 2.2. We recapitulate the QCD predictions of the hadronic widths by Kwong and Rosner, ${ }^{22}$ using the lowest order QCD calculations of Barbieri et al., ${ }^{30}$ they are:

$$
\begin{aligned}
& \Gamma_{h a d}\left(\chi_{b 2}^{\prime}\right)=145(153) \mathrm{keV} \\
& \Gamma_{h a d}\left(\chi_{b 1}^{\prime}\right)=51 \mathrm{keV} \\
& \Gamma_{h a d}\left(\chi_{b 0}^{\prime}\right)=542(866) \mathrm{keV} .
\end{aligned}
$$

The widths of the $\mathrm{J}=2$ and $\mathrm{J}=0$ states including the first order corrections are given in parentheses.

The ratio of the widths of the $\mathrm{J}=0$ and $\mathrm{J}=2$ states is equal to $15 / 4 \times\left(1+10.2 \alpha_{s} / \pi\right) /\left(1+1.0 \alpha_{s} / \pi\right)^{22}$ Thus we note that a precise measurement would yield a new test of QCD, i.e. provide an independent way of determining $\alpha_{s}$.

The current experimental the value for this ratio is $5.12 \pm$ 2.45. This accuracy is insufficient to distinguish between leading order, 3.75, and next to leading order, 5.65. This is because of insufficient statistics in the $\chi_{b 0}^{\prime}$ channel. The present CUSB result is based on $(4.1 \pm 2.7)$ events in the $\Upsilon(2 S) \gamma \gamma$ channel and $(8.1 \pm 3.7)$ events in the $\Upsilon(1 S) \gamma \gamma$ channel. From a $1.5 \times 10^{7}$ sample of $\Upsilon(3 S)$ events, one would collect more than 500 exclusive events in the $\chi_{b 0}^{\prime}$ channel, sufficient to reduce the error on the ratio to $\approx 5 \%$ of its value. Similarly, we will be able to measure the ratio for the $\Upsilon(2 S)$ decays, which 
has not and will not be measured until a $B$ factory comes into existence.

\section{Gluon vs Quark Fragmentation}

The combination of excellent photon energy resolution, $\pi^{0}$ reconstruction efficiency, and large data samples would allow us to tag inclusive events in $\Upsilon(3 S)$ decays which have 85 and $122 \mathrm{MeV}$ photons in the them. Depending on the eventual $\pi^{0}$ reconstruction efficiency, one could obtain a relatively pure sample of tagged $\chi_{b 0,2}^{\prime}$ events. These states are expected to decay principally into two gluons, allowing us to compare the fragmentation of two gluon decays with two quark decays (from continuum decays).

\section{Hadronic Transitions}

To date, no hadronic transitions other than $\Upsilon(3 S, 2 S) \rightarrow \pi \pi \Upsilon(2 S, 1 S)$ have been seen, and the present upper limits for transitions via the $\eta$ are at the level of a few $\times 10^{-3}$. Scaling from the $\psi(2 S) \rightarrow \eta J / \psi$, one expects that the branching ratio for $\Upsilon(2 S) \rightarrow \eta \Upsilon(1 S)$ decay will be $\approx 0.1 \%$, which is somewhat less than an order of magnitude smaller than present limits. Similarly, the $\Upsilon(3 S) \rightarrow \eta \Upsilon(1 S)$ decay is expected to have a somewhat smaller branching ratio $(0.02 \%)$. With an increase over the present statistics of between a factor of 10 to 100 , we should be able to observe these decays and check the multipole expansion description of hadronic transitions.

Radiative $\Upsilon$ Decays and Search for New Particles

A $B$ factory is an excellent laboratory in which to search for new particles, rare transitions, and measure $\alpha_{s}$ because of the vast $\left(\approx 10^{8}\right)$ data sample of $\Upsilon(1 S)$ events one could obtain there. In the following sections, we discuss some of the $\Upsilon$ radiative decay physics that can be done.

$B_{\gamma g g}$ and $\alpha_{s}$

These large data samples will be able to unambiguously determine the shape of th $e$ high energy photon spectrum from $\Upsilon \rightarrow \gamma g g$ decays. One can also expect a very significant improvement in the accuracy of the $B R(\Upsilon \rightarrow \gamma g g)$ measurement which is presently known to $10 \%$ of its value. 


\section{Radiative $\Upsilon$ Decays to Light Mesons}

As can be seen in Table 6 , we have only just begun to reach the levels of sensitivity required to see $\Upsilon \rightarrow \gamma+$ light mesons decays. If the branching ratios are indeed as expected, a large number of possible decays will be seen with a few hundred events in each channel, enough to provide stringent tests of the theoretical predictions.

\section{Search for Light Neutral Higgs}

Finding the Higgs is perhaps the central problem in high energy physics today. A light neutral Higgs has not yet been unambiguously ruled out, so one must consider methods to search for them. In fact supersymmetric theories tend to favor $^{32}$ a light Higgs. One of the methods that has been successfully employed is to search for radiative decays, $\Upsilon \rightarrow$ $\gamma+H$. The expected branching ratio has been calculated by Wilczek $^{33}$ and by Weinberg ${ }^{34}$ who find

$$
B R(\Upsilon \rightarrow \gamma+H)=B R_{\mu \mu} \times \frac{G_{F} M_{b}^{2}}{\sqrt{2} \pi \alpha}\left(1-\frac{M_{H}^{2}}{M_{\Upsilon}^{2}}\right) x^{2} f_{r a d}
$$

where $x$ is the ratio of vacuum expectation values for the Higgs fields (or $x=1$ in the minimal Standard Model) and where $f_{\text {rad }} \approx 0.5$ is the radiative correction factor. ${ }^{35}$ Thus one expects $B R(\Upsilon \rightarrow \gamma H) \approx 10^{-4}$. That level of sensitivity has been reached now, but with these large data samples, one should be able to push the upper limits down by at least an order of magnitude. That should finally place the experimental upper limits well beyond the reach of ever changing radiative correction effects, and thus unequivocally rule out a light Standard Model Higgs below the $\approx 7 \mathrm{GeV}$ level or perhaps even find it.

Another interesting issue has been raised by Drees and Hikasa ${ }^{36}$ who point out that if the Higgs should 1 ie near the $\Upsilon$ or $\chi_{b}$, then one should expect considerable mixing effects, which could be searched for in the decays $\Upsilon(3 S) \rightarrow \gamma \chi_{b}^{\prime} \rightarrow$ $\gamma \tau \tau$. In fact for $M_{H} \approx 10 \mathrm{GeV}$ this may be one of the only ways to search for it. 
High .Statistics Studies of $B_{\mu \mu}, B_{\tau \tau}$, E1 rates...

The high statistics obtained at the proposed $B$ factory would also enhance the "bread and butter" physics by providing the statistics to do high precision studies of already relatively well measured quantities. The gains are obvious, and we do not enumerate them here, except for one case that cannot be done without these high statistics.

$B_{\mu \mu}$ and $\alpha_{s}$

$B_{\mu \mu}$ is well measured, and will probably not benefit much from very high statistics until theory catches up with the existing experimental precision. However there are other precision tests such as $\mu-\tau$ universality that can be addressed (see next section).

$\mu-\tau$ Universality and a Compositeness Scale

The question of universality among generations can provide a useful probe for physics beyond the Standard Model. Here we investigate the sensitivity attainable in a comparison of $\Upsilon \rightarrow \tau^{+} \tau^{-}$with $\Upsilon \rightarrow \mu^{+} \mu^{-}$. The immediate theoretical impetus for this study came in the claim by Pati and Stremnitzer ${ }^{37}$ that large differences might be observed in toponium decays due to compositeness, where they stress the possibility that compositeness may show up at a lower scale in the third family than in the first two. It is expected that the effects will be substantially smaller in $\Upsilon$ decays, but the possibility of very high statistics experiments makes the question worth studying. Of course the question of universality is of general interest, but we use the Pati and Stremnitzer model as a guide for where the interesting region lies.

The effect of compositeness on $\Upsilon \rightarrow \tau^{+} \tau^{-}$

In this section, we reapply Pati \& Stremnitzer's calculation to the upsilon system. Without resorting to a specific model, there is no very precise prediction, so we give a range of predictions, as a function of compositeness parameters. This range should not be construed as covering all compositeness ideas, but at least is interesting.

First, we generalize the equations in Ref. 37 to apply to the upsilon system as well as the toponium system. Define
Compositeness may show up at a lower scale in the third family. Little experimental constraint exists here. 
the ratio:

$$
R \equiv \frac{\Gamma\left(V \rightarrow \tau^{+} \tau^{-}\right)}{\Gamma\left(V \rightarrow \mu^{+} \mu^{-}\right)}
$$

where $V$ is either $\Upsilon$ or $\theta$. There are two diagrams which we consider for the $\mu^{+} \mu^{-}$decay, corresponding to quark-antiquark annihilation through a photon or a $Z^{0}$. A third diagram appears in addition for the $\tau^{+} \tau^{-}$decay, as a consequence of shared components in the third generation quarks and leptons according to the compositeness model. The ratio $R$ is conveniently expressed in the form:

$$
R=\frac{\left|\lambda_{\tau}\right|^{2}+\left|\lambda_{\tau}^{\prime}\right|^{2}}{\left|\lambda_{\mu}\right|^{2}+\left|\lambda_{\mu}^{\prime}\right|^{2}}
$$

We generalize Pati and Stremnitzer's expressions for $\lambda$ and $\lambda^{\prime}$ to apply to both toponium and bottomonium decays, as well as include a slight modification for the hypercolor term suggested by $B$. Ward:

$$
\begin{aligned}
& \lambda_{\ell}=-e_{q} \frac{e^{2}}{s}-\frac{G_{F} m_{Z}^{2}}{2 \sqrt{2}} \frac{2\left(g_{R q}+g_{L q}\right)\left(-1+4 \sin ^{2} \theta_{W}\right)}{D_{Z}(s)} \\
& \quad- \begin{cases}0 & \text { if } \ell \neq \tau \\
\frac{\kappa^{2}}{\Lambda^{2}-s} & \text { if } \ell=\tau\end{cases} \\
& \lambda_{\ell}^{\prime}=\frac{G_{F} m_{Z}^{2}}{2 \sqrt{2}} \frac{2\left(g_{R q}+g_{L q}\right)}{D_{Z}(s)},
\end{aligned}
$$

where $D_{Z}(s)=s-m_{Z}^{2}+i m_{Z} \Gamma_{Z}, s=m_{V}^{2}$ is the square of the vector meson mass, and the hypercolor force is assumed to conserve parity. The hypercolor term is arranged so that the sign of the strength parameter, $\kappa^{2}$, is consistent with Stremnitzer and Pati's second paper, ${ }^{38}$ and opposite to the sign in their first paper. ${ }^{37}$ With the sign convention as chosen here, Stremnitzer and Pati suggest that $\kappa^{2}$ is most naturally positive, though this is not required. The quark charge is $e_{q}$, and $-2\left(g_{R q}+g_{L q}\right)=2 I_{3 q}^{W}-4 e_{q} \sin ^{2} \theta_{W}$. It is convenient to also express $R$ in the form $R=1+\delta$ with:

$$
\delta=\frac{\kappa^{2}}{\Lambda^{2}-m_{V}^{2}}\left(\frac{\kappa^{2}}{\Lambda^{2}-m_{V}^{2}}+2 \Re \lambda_{\mu}\right) /\left(\left|\lambda_{\mu}\right|^{2}+\left|\lambda_{\mu}^{\prime}\right|^{2}\right) .
$$

There are two unknown parameters associated with the 
compositeness picture which are needed in an evaluation of $R$ : $(i) \kappa^{2}$ is a strength parameter associated with the compositeness force. By analogy with the ordinary strong force (QCD), it is argued ${ }^{37}$ that one expects $\left|\kappa^{2}\right| \simeq 1$ to 10 . (ii) $\Lambda$ is a compositeness size parameter for the $\tau-b-t$ family and is related to $\Lambda_{H C}$, a scale parameter for the hypercolor gauge force which binds ${ }^{39}$ this family. It is argued ${ }^{37,39}$ that, in a world with three families, this scale cannot be larger than a few $\mathrm{TeV}$ if the compositeness idea is a correct approach for the masses in this family. However, the effective size parameter $\Lambda$ may be different for different members of the family.

To calculate the expectation for $\Upsilon$ decays, we tune the calculation to agree with Pati \& Stremnitzer's results for toponium. The $Z^{0}$ diagram is not a serious contribution at the $\Upsilon$, nor is the mass of the $\Upsilon$ (compared with $\Lambda$ ), though they are included in the calculation. Neglect of these contributions yields the simplified formula:

$$
\begin{aligned}
R_{\Upsilon} & \equiv \frac{\Gamma\left(\Upsilon \rightarrow \tau^{+} \tau^{-}\right)}{\Gamma\left(\Upsilon \rightarrow \mu^{+} \mu^{-}\right)} \\
& =1+\frac{9}{16 \pi^{2}} \frac{\kappa^{2}}{\alpha}\left(\frac{m_{\Upsilon}}{\Lambda}\right)^{2}\left[\frac{\kappa^{2}}{\alpha}\left(\frac{m_{\Upsilon}}{\Lambda}\right)^{2}-\frac{8 \pi}{3}\right]
\end{aligned}
$$

In practice, we need to apply a correction for the non-zero lepton masses, since we need to deal with rather small deviations from $\mu-\tau$ universality, as we shall see. Since the parity violating contribution may be neglected, we may simply multiply the value of $R$ in formula (7.5) by

$$
f_{\tau / \mu}=\frac{v_{\tau}\left(3-v_{\tau}^{2}\right)}{v_{\mu}\left(3-v_{\mu}^{2}\right)}=0.99227_{-0.00007}^{+0.00005}
$$

where velocities appropriate to $\Upsilon$ decays have been used.

We thus obtain a family of curves for $R_{\Upsilon}$ as a function of $\Lambda$ and $\kappa^{2}$, as shown in Figure 7.5. 
Figure 7.5 $R_{\Upsilon}$ as a function of $\Lambda$ for different values of $\kappa^{2}$. The mass of the $\Upsilon$ is taken to be $9.46 \mathrm{GeV}$.

Measurement of $R_{\Upsilon}$ to $\$ 1 \%$ is the goal for interesting physics reach.

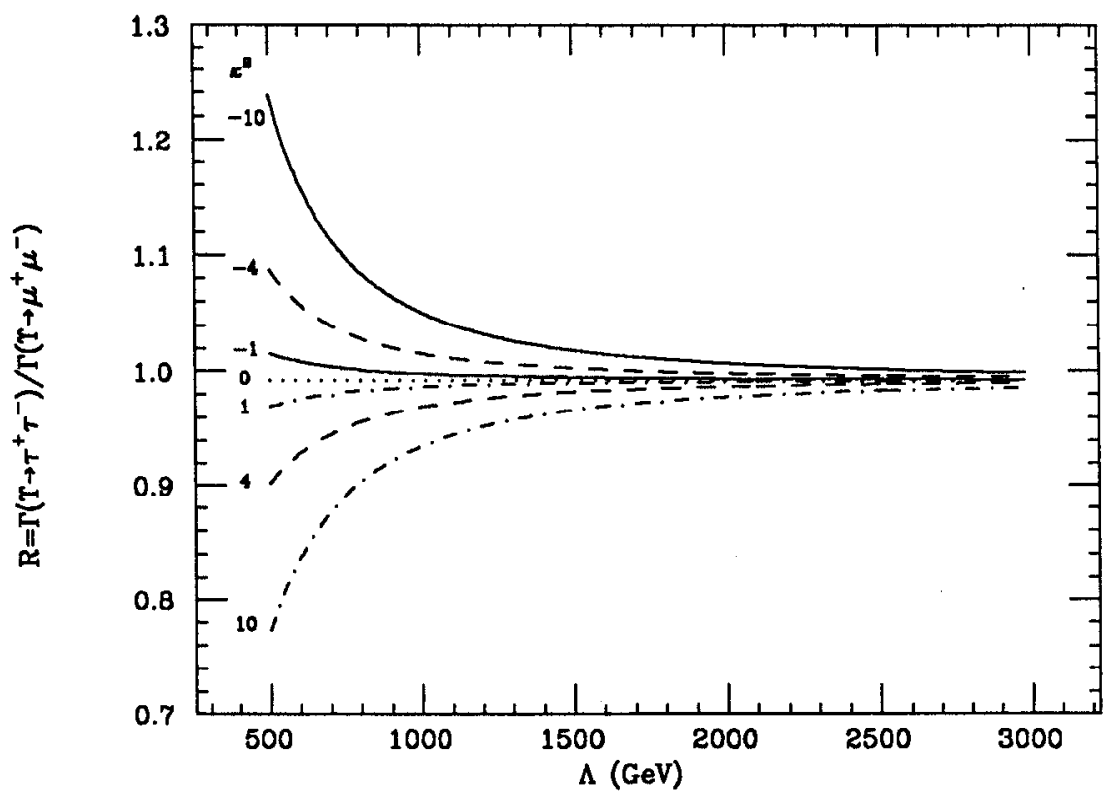

The conclusion from this figure is that an experiment to search for compositeness in $\Upsilon \rightarrow \tau^{+} \tau^{-}$decays with a precision of $\lesssim 1 \%$ in the measurement of $R_{\Upsilon}$ could be very interesting.

Experimental measurement of $R_{\Upsilon}$

In considering the experimental measurement of $R_{\Upsilon}$, let us first look at the current situation. The 1988 world averages are: ${ }^{40}$

$$
\begin{aligned}
& B\left(\Upsilon \rightarrow \mu^{+} \mu^{-}\right)=2.6 \pm 0.2 \% \\
& B\left(\Upsilon \rightarrow \tau^{+} \tau^{-}\right)=3.0 \pm 0.4 \% .
\end{aligned}
$$

Thus, the current world average for the ratio, neglecting possible correlations between these measurements, is $R_{\Upsilon}=1.15 \pm$ 0.18 . This is about one standard deviation above the value expected in the Standard Model (0.992). The preliminary world averages for the 1990 edition of the Review of Particle Properties (as obtained from the PARTICLES database in SPIRES) are $B\left(\Upsilon \rightarrow \mu^{+} \mu^{-}\right)=2.61 \pm 0.11 \%$ and $B(\Upsilon \rightarrow$ $\left.\tau^{+} \tau^{-}\right)=2.97 \pm 0.35 \%$, yiclding $R_{\Upsilon}=1.14 \pm 0.14$, with the 
same conclusion as from the data available in 1988. Unfortunately, the experimental errors are too large to expect to see evidence for compositeness, or to put especially interesting constraints on the model-builders.

However, given the high statistics that may become available for $\Upsilon$ physics, measurements at the level of $\lesssim 1 \%$ might be attainable in the future. A measurement of such precision requires some care. A good approach, in terms of control of systematic effects, is one in which the $\mu^{+} \mu^{-}$and $\tau^{+} \tau^{-}$are measured at the $\Upsilon$ and in the continuum just below the resonance. Simultaneous measurement of $\tau \tau$ and $\mu \mu$ is important so that sensitivity to knowledge of the accumulated luminosity may be suppressed or eliminated.

Let $N_{\tau \tau}$ be the number of $\tau$-pair events and $N_{\mu \mu}$ the number of $\mu$-pair events observed on the $\Upsilon$, and $M_{\tau \tau}, M_{\mu \mu}$ be the corresponding observed numbers in the continuum below the $\Upsilon$. The efficiencies are assumed to be the same on and off the resonance. Also, $r_{\sigma_{\ell}}$ is the ratio of the continuum $\ell^{+} \ell^{-}$cross section on resonance to below resonance $\left(r_{\sigma_{\ell}} \approx 1\right.$ is approximately the ratio of the inverse squares of the energies), and $r_{L}$ is the ratio of the accumulated luminosity on resonance to below resonance.

With these definitions, we may write the measured value of $R_{\Upsilon}$ in this experiment as:

$$
R_{\Upsilon}=f_{\tau / \mu} \frac{N_{\tau \tau} / M_{\tau \tau}-r_{\sigma_{\tau}} r_{L}}{N_{\mu \mu} / M_{\mu \mu}-r_{\sigma_{\mu}} r_{L}}
$$

The $f_{\tau / \mu}$ which appears in this expression is to be evaluated at the continuum point. Propagation of errors yields the estimated error:

$$
\begin{aligned}
\frac{\delta_{R_{\Upsilon}}}{R_{\Upsilon}} & =\left\{\left(\frac{\delta_{f_{\tau / \mu}}}{f_{\tau / \mu}}\right)^{2}+\left(\frac{N_{\tau \tau}}{N_{\tau \tau}-r_{\sigma} r_{L} M_{\tau \tau}}\right)^{2}\left(\frac{1}{N_{\tau \tau}}+\frac{1}{M_{\tau \tau}}\right)\right. \\
& +\left(\frac{N_{\mu \mu}}{N_{\mu \mu}-r_{\sigma} r_{L} M_{\mu \mu}}\right)^{2}\left(\frac{1}{N_{\mu \mu}}+\frac{1}{M_{\mu \mu}}\right) \\
+ & {\left.\left[\frac{\left(N_{\tau \tau} M_{\mu \mu}-N_{\mu \mu} M_{\tau \tau}\right)}{\left(N_{\tau \tau}-r_{\sigma} r_{L} M_{\tau \tau}\right)\left(N_{\mu \mu}-r_{\sigma} r_{L} M_{\mu \mu}\right)}\right]^{2}\left[\left(\frac{\delta_{r_{L}}}{r_{L}}\right)^{2}+\left(\frac{\delta_{r_{\sigma}}}{r_{\sigma}}\right)^{2}\right]\right\}, }
\end{aligned}
$$

where we have used the simplification $r_{\sigma_{\tau}} \approx r_{\sigma_{\mu}}=r_{\sigma}$. We 
We propose effectively a doubleratio measurement of $R_{\Upsilon}$, in which systematics cancel, yielding a statistics-limited experiment. will neglect the uncertainty in $f_{\tau / \mu}$. In the limit $R_{\Upsilon}=1$ (i.e., $\left.N_{\tau \tau} / M_{\tau \tau}=N_{\mu \mu} / M_{\mu \mu}\right)$, the estimated error reduces to:

$$
\frac{\delta_{R_{\Upsilon}}}{R_{\Upsilon}}=\frac{N_{\mu \mu}}{N_{\mu \mu}-N_{\mu \mu}^{c}} \sqrt{\frac{1}{N_{\tau \tau}}+\frac{1}{M_{\tau \tau}}+\frac{1}{N_{\mu \mu}}+\frac{1}{M_{\mu \mu}}}
$$

The important point here is that by using the below resonance measurement, we drastically reduce the contribution from systematic effects - to an excellent approximation, we have a statistics-limited experiment.

We may now make numerical estimates of the amount of integrated luminosity required for a given statistical error in $R_{\Upsilon}$. For the numerical estimates, we assume $B(\Upsilon \rightarrow$ $\left.\mu^{+} \mu^{-}\right)=B\left(\Upsilon \rightarrow \tau^{+} \tau^{-}\right)=0.03$ and $\sigma\left(e^{+} e^{-} \rightarrow \Upsilon\right)=18 \mathrm{nb}$ (observed value at CESR, including beam spread and radiative effects). Also for the sake of numerical estimates, we assume that the continuum $\mu$-pair and $\tau$-pair cross sections are given by the QED single photon cross section, $1 \mathrm{nb}$, at the $\Upsilon$ energy. For simplicity, we will also take $\epsilon_{\mu}=\epsilon_{\tau}$, though this is not required.

The optimum strategy is to divide the running time so as to observe equal numbers of $\mu$-pairs at the two energy points: $N_{\mu \mu}=M_{\mu \mu}$ and (in limit $R_{\Upsilon}=f_{\tau / \mu}$ ) $N_{\tau \tau}=M_{\tau \tau}$. For a desired error in $R_{\Upsilon}$, on the $\Upsilon$ point, we must obtain:

$$
N_{\Upsilon}^{\text {produced }} \approx 4 \frac{1+N_{\mu \mu}^{c} / N_{\mu \mu}^{\Upsilon}}{\epsilon_{\tau} B\left(\Upsilon \rightarrow \mu^{+} \mu^{-}\right)}\left(\frac{R_{\Upsilon}}{\delta_{R_{\Upsilon}}}\right)^{2}
$$

where $N_{\ell \ell}^{c}$ refers to the non-resonant continuum contribution to the observed numbers (likewise $N_{\ell \ell}^{\Upsilon}$ refers to the resonant contribution to the observed numbers), and $\epsilon_{\ell}$ is the efficiency to observe $\ell^{+} \ell^{-}$, including $\ell$ decay modes and detector limitations. Off-resonance, we must run a factor of $\sim$ $1+N_{\mu \mu}^{\Upsilon} / N_{\mu \mu}^{c} \sim 1.5$ times longer (for the cross section assumptions stated earlier). We summarize the required datasets in the following Table: 


\begin{tabular}{|c|c|}
\hline$\delta_{R_{\Upsilon}} / R_{\Upsilon}$ & Required equivalent $N_{\Upsilon}^{\text {produced }}$ \\
\hline 0.01 & $10^{7} / \epsilon_{\tau}$ \\
\hline 0.001 & $10^{9} / \epsilon_{\tau}$ \\
\hline
\end{tabular}

Assuming proper care is taken to insure equivalent running on and off resonance, the experiment should be statisticslimited. A measurement at the level of a $1 \%$ measurement of $R_{\Upsilon}$ requires a relatively small investment in running time for a $B$-factory. Note that the statistics required for a $0.1 \%$ error is also within reach of a $B$ factory of the luminosity under consideration.

\section{Backgrounds}

The design of the experiment is such that backgrounds tend to cancel out. The only potential worry is backgrounds from other decays of the $\Upsilon$ itself, since continuum contributions cancel. We note that no exclusive decays of the $\Upsilon(1 S)$ have been observed other than the lepton pairs. Backgrounds due to cross-over in the lepton decay channels are not a serious problem even if they occur, and even we have only a rough idea how large they are. This is because this source will occur in the same way on the continuum, and so the uncertainty in this source cancels to a good approximation. We need only be concerned about backgrounds from $\Upsilon$ decay processes that are not compensated by identical backgrounds in the continuum.

The limits on exclusive branching ratios of the $\Upsilon(1 S)$, which could contribute backgrounds exist for a few channels, and are typically of the order of $10^{-4}$, already considerably smaller than the branching ratio to lepton pairs. Thus, tails in these low multiplicity decay modes are unlikely to pose much of a problem. Fortunately, with a high luminosity machine, we will be able to study the background sources at the same time as we collect the data for the measurement.

\section{Summary}

The prospect of a precision test of $\mu-\tau$ universality in $\Upsilon$ decays is very promising. Without resorting to a specific compositeness model, a measurement of $R_{\Upsilon}$ at the level of $\lesssim 1 \%$
A $1 \%$ measurement is easy for a $B$-factory, the required statistics for a $0.1 \%$ measurement is also attainable.

The largest background sources will not contribute to the uncertainty in $R_{\Upsilon}$. Remaining backgrounds from $\Upsilon$ decays are expected to be negligible, and can be examined in the same experiment. 
The ability to tag $B^{*}$ events via their $50 \mathrm{MeV}$ photon may prove to be important in $C P$ violation studies probes a new region in the third family. Such a measurement appears to offer the potential of an exciting and fundamental discovery, and at least will provide useful constraints

Spectroscopy Above the b-Flavor Threshold

Although the principal focus of this section has been on the spectroscopy accessible from bound $b \bar{b}$ states, one should address the question of spectroscopy above the $b$ flavor threshold. In particular, with excellent photon energy resolution and low photon energy cutoffs we would accurately measure the $B^{*} \rightarrow B+\gamma$ photon spectrum (where $E_{\gamma} \approx 50 \mathrm{MeV}$ has already been measured by CUSB), and from the shape of the spectrum one can study $B_{s}^{(*)}$ production $v s$ energy. This would allow for a determination of the optimal energy at which to run in order to observe $B_{s}-\bar{B}_{s}$ mixing.

\subsection{Detector Constraints}

The physics that we have discussed in previous sections relies on having an excellent detector capable not only of precision charged track measurements, but also achieving the highest possible energy resolution for photons.

Angular Resolution

We have carried out several studies of the necessary angular resolution required by the detector in order not to compromise the intrinsic electromagnetic energy resolution. Figure 7.6 shows the contours for various contributions to the energy resolution due to angular resolution alone. These studies were done assuming asymmetric collisions of 9 on $3 \mathrm{GeV}$. If one maintains an angular resolution of better than $20 \mathrm{mr}$, its contribution to the energy resolution is negligible. A similar study of the angular resolution contribution to the energy resolution is shown in Figure 7.7. The resolution shown here is only from angular smearing with no contribution from photon energy resolution. The four photon lines shown in Figure 7.7 correspond to the transitions $\Upsilon(3 S) \rightarrow \chi_{b(J=2,1)}^{\prime}$ followed by $\chi_{b(J=2,1)}^{\prime} \rightarrow \Upsilon(2 S)$. The two higher energy lines have some doppler smearing, from recoil of the $\chi_{b}^{\prime}$. All lines are clearly resolved even with an angular resolution of $30 \mathrm{mr}$, it is only at 
the very poorest resolutions $(>50 \mathrm{mr})$ that the "unboosted" energy resolution begins to deteriorate.

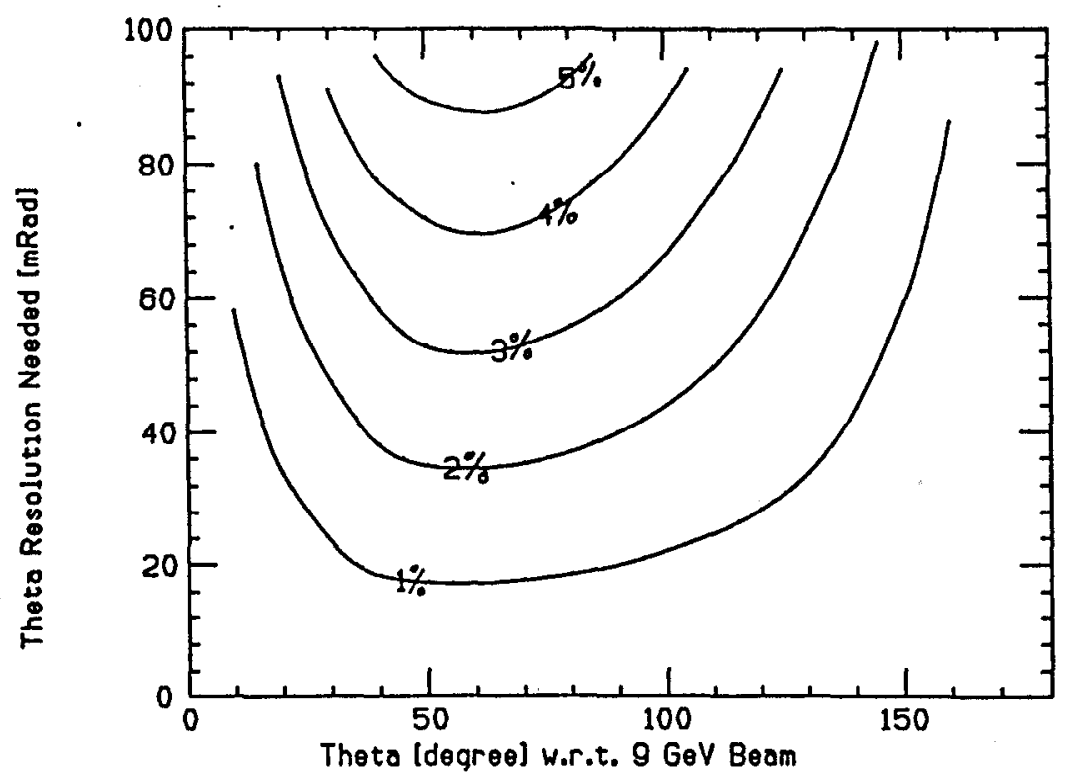

The angular resolution is of course determined by the number of calorimeter elements. We can make a very simple estimate of the number of elements required to achieve 10 and $20 \mathrm{mr}$, assuming that the full solid angle is covered, and that there is no energy sharing between elements (this is the most pessimistic case). Under those conditions, the rms angular resolution is $1 / \sqrt{12}$ times the actual angular opening of the calorimeter towers. The number of detector elements is shown in Table 7.8 .

Table 7.8 Minimum number of calorimeter elements.

\begin{tabular}{|l|c|c|}
\hline & $\delta \theta=20 \mathrm{mr}$ & $\delta \theta=10 \mathrm{mr}$ \\
\hline No. of detector elements & 2,600 & 10,500 \\
\hline
\end{tabular}

Figure 7.6 Energy resolution contours vs angular resolution.

An asymmetric $B$ factory is as good for doing $\Upsilon$ spectroscopy as a symmetric machine 
Figure 7.7 Photon spectrum for $\Upsilon(3 S) \rightarrow \chi_{b(J=2,1)}^{\prime}$ vs angular resolution.

Given the proper detector, $\Upsilon$ physics is as easily done at an asymmetric machine as at a symmetric machine

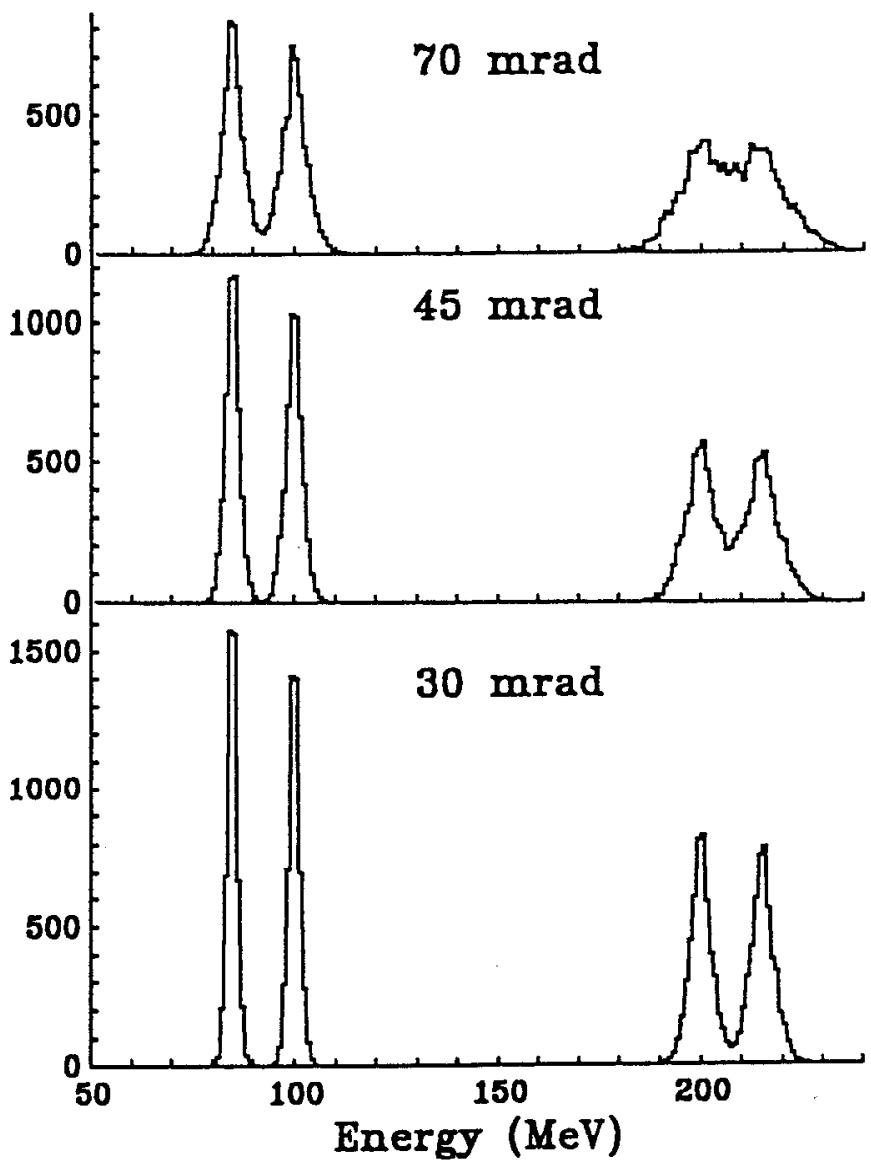

In conclusion, we find that for any reasonable detector being considered, the Doppler smearing produced by an asymmetric $e^{+} e^{-}$machine can be easily and effectively removed. $\Upsilon$ physics at an asymmetric machine is as easily done as at a symmetric machine.

\section{$\underline{\text { Energy Resolution }}$}

The question of energy resolution is more critical to the physics we have considered. Much of the physics demands excellent photon energy resolution. It includes the study of D state spectroscopy, where the splittings are very small $(\approx 6 \mathrm{MeV}), \chi_{b}$ and $\chi_{b}^{\prime}$ tagging studies, and $\pi^{0}$ reconstruction (where one needs to maintain good mass resolution). 


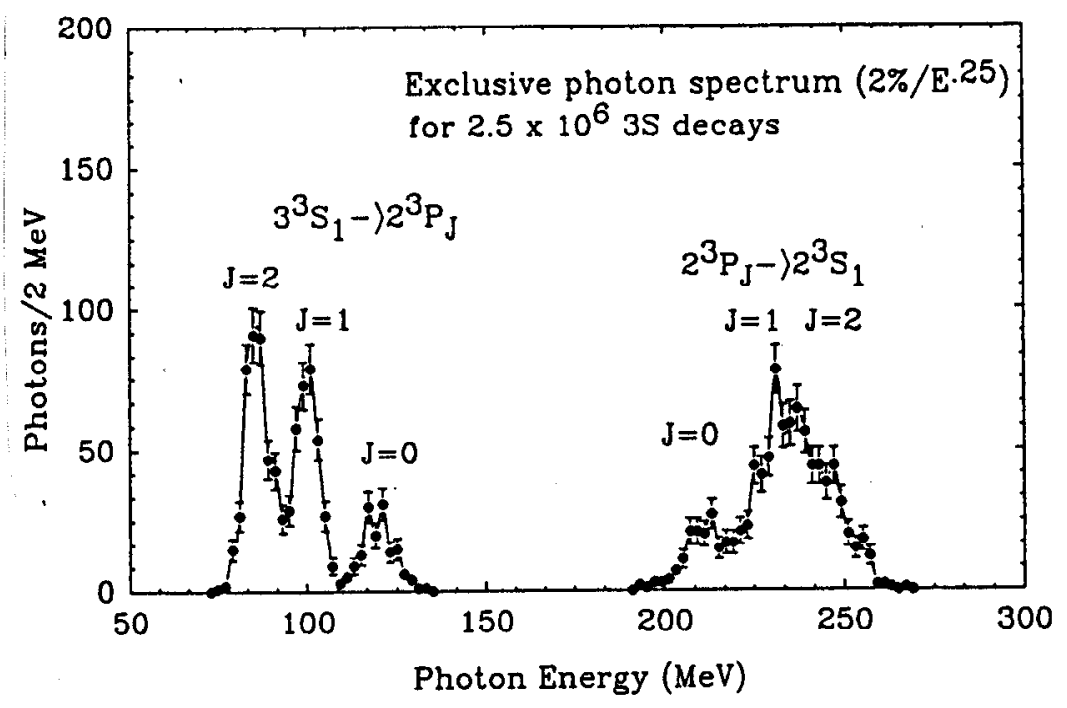

Figure 7.8 Photon spectrum for $\Upsilon(3 S) \rightarrow \chi_{b(J=2,1,0)}^{\prime}$ and $\chi_{b}^{\prime} \rightarrow \Upsilon(2 S)$ decays with $\sigma_{E} / E=2 \% / \sqrt[4]{E} \oplus 1 \%$

As an example of the effect of resolution on $\chi_{b}$ studies, Figure 7.8 shows the photon spectrum assuming a resolution of $\sigma_{E} / E=2 \% / \sqrt[4]{E} \oplus 1 \%$. While this resolution appears quite adequate to measure the $\Upsilon(3 S) \rightarrow \chi_{b}^{\prime}$ lines, the $\chi_{b}^{\prime} \rightarrow \Upsilon(2 S)$ lines are no longer fully resolved. The situation is aggravated for the $D$ state lines, where the splittings are significantly smaller. Improving the resolution to $1 \%$ instead of $2 \%$ leads to the spectrum shown in Figure 7.9. Note that now the $\chi_{b}^{\prime} \rightarrow \Upsilon(2 S)$ lines are fully resolved, and that the $\mathrm{D}$ state lines are partially resolved as was shown in Figure 7.3.

The energy resolution plays a very important role in $\pi^{0}$ reconstruction. Previous $B$ factory workshops have emphasized the need for excellent. electromagnetic calorimetry in order to achieve the very good $\pi^{0}$ resolution that is necessary not only for $\Upsilon$ physics, wherc one needs it to reduce photon backgrounds in inclusive photon spectra, but also in $B$ physics where it is quite as important as excellent charged particle resolution when reconstructing $B$ particles. 
Figure 7.9 Photon spectrum for $\Upsilon(3 S) \rightarrow \chi_{b(J=2,1,0)}^{\prime}$ and $\chi_{b}^{\prime} \rightarrow \Upsilon(2 S)$ decays with $\sigma_{E} / E=1 \% / \sqrt[4]{E}$

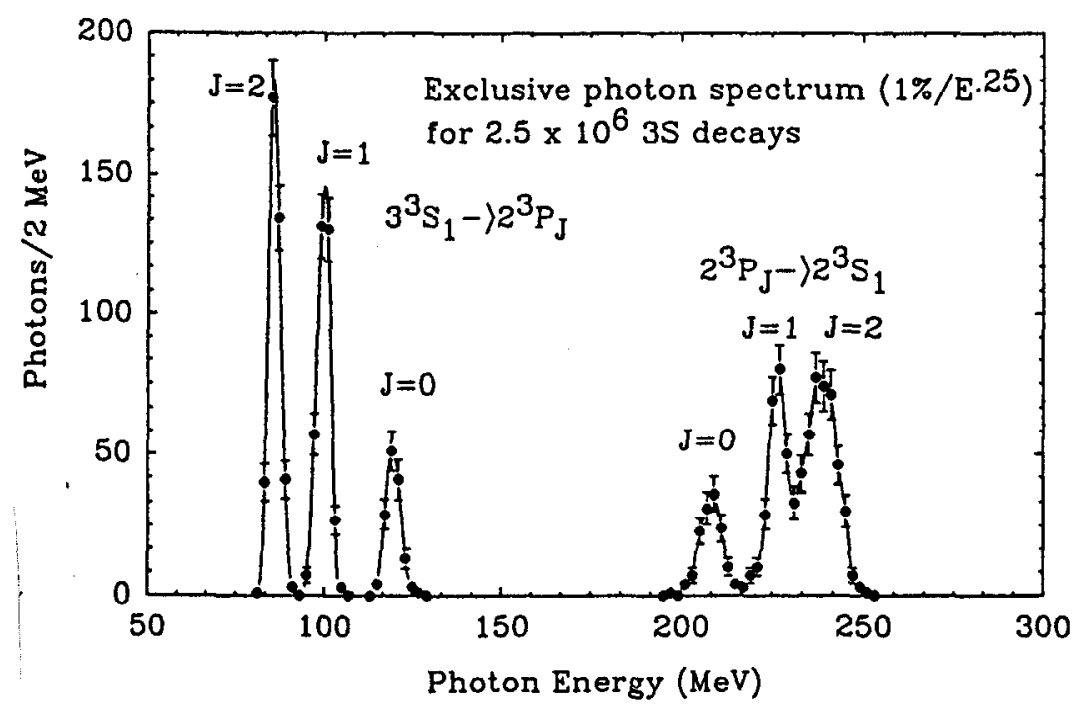

Figure 7.10 shows a comparison ${ }^{41}$ of what a modern CLEOII like detector should be capable of in terms of mass resolution (under ideal assumptions such as no lower $E_{\gamma}$ cutoff, and no noise in the crystal readout), and Figure 7.11 shows what a superb calorimeter ${ }^{41}$ could achieve with an energy resolution of $1 \%$ at $100 \mathrm{MeV}$. The benefits are self-evident. In actual use real detectors have other limitations imposed on them, e.g., the actual $\pi^{0}$ reconstruction achieved with the first run of the CLEO-II endcaps was significantly poorer than that shown in Figure 7.10. 


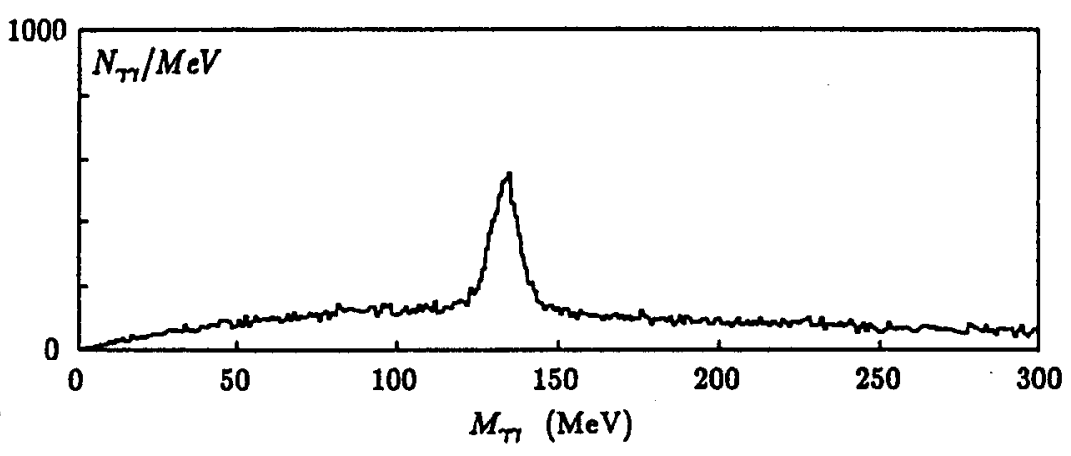

Figure 7.10 Reconstructed $\pi^{0}$ spectrum in a typical crystal calorimeter.

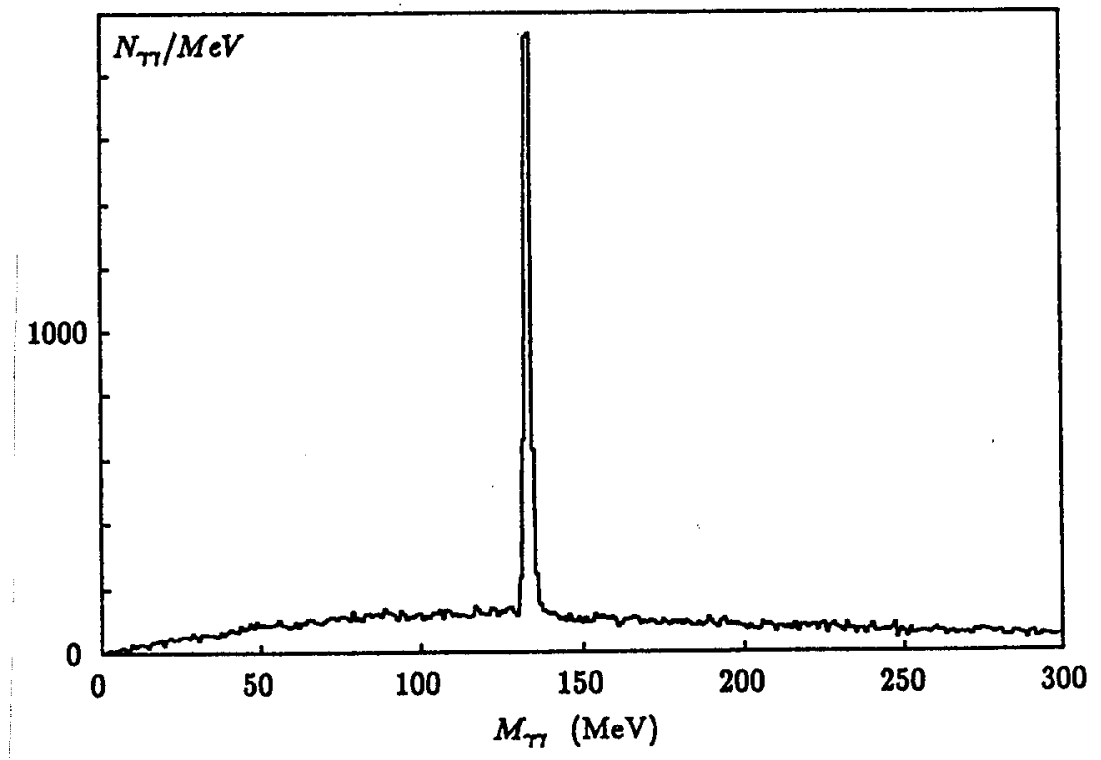

Figure 7.11 Reconstructed $\pi^{0}$ spectrum in an optimal calorimeter. 
Figure 7.12 Reconstruction efficiency for $\pi^{0}$ vs minimum $E_{\gamma}$ cutoff for optimized calorimeter.

Figure 7.13 Fraction of $B$ events in which all $\pi^{0}$ 's are reconstructed.
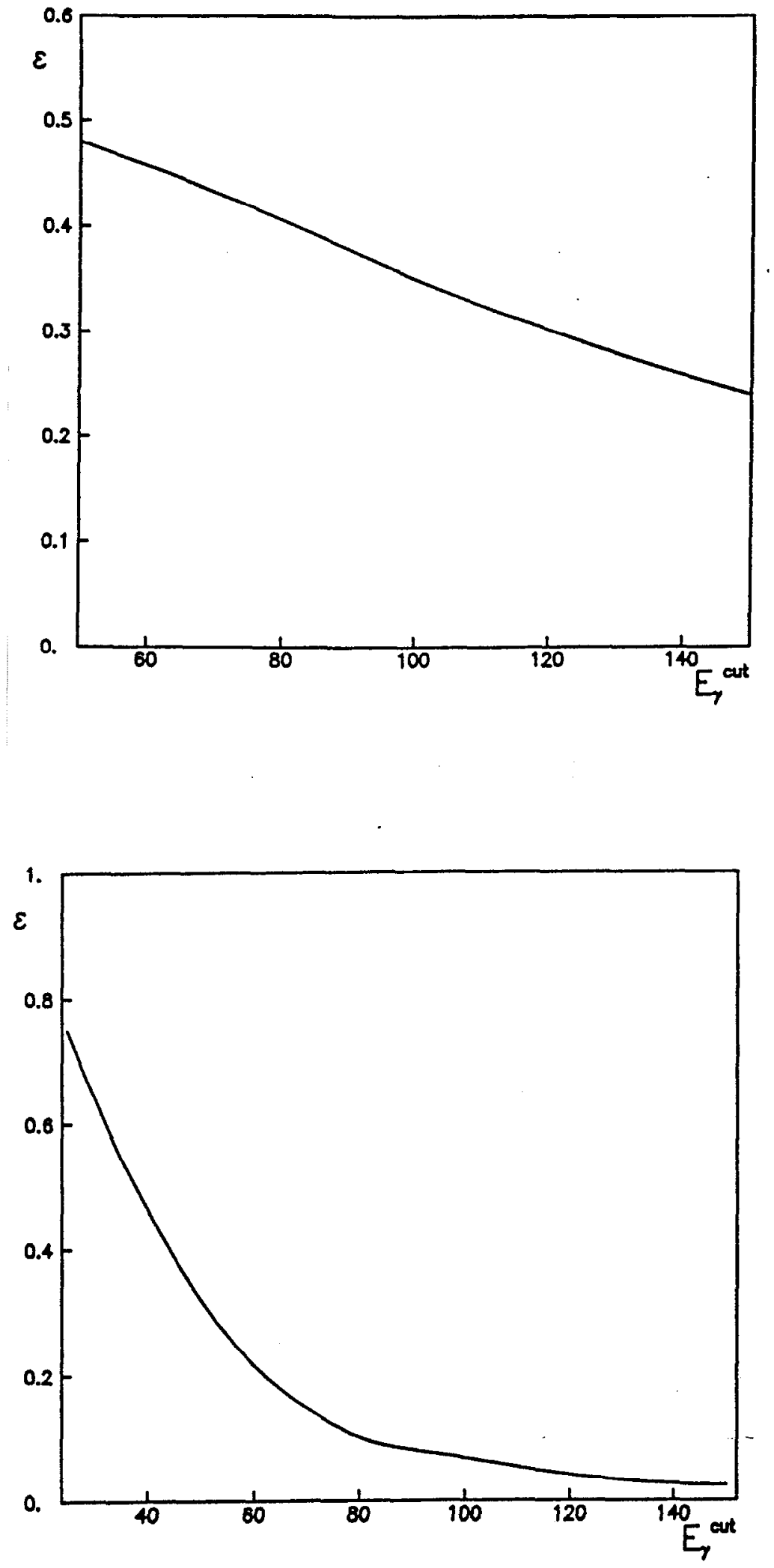


\section{Minimum Photon Energy Cutoff}

One of the constraints imposed on a real calorimeter is that sufficiently low energy photons, below some value $E_{\gamma}^{\text {low }}$, are not observed. Other $B$ factory studies, ${ }^{42}$ assuming the very best possible electromagnetic calorimeters, have shown that one pays dearly in $\pi^{0}$ reconstruction efliciency as a function of $E_{\gamma}^{\text {low }}$. Figures 7.12 and 7.13 from ref. 42 show that one must pay close attention to the low energy photon cutoff. For a cutoff of $50 \mathrm{MeV}$, the efficiency for reconstructing a photon in an average $B$ event is reduced to $50 \%$; the probability of reconstructing all $\pi^{0}$ 's drops to $30 \%$.

\subsection{CONClusions}

We find that if care is paid to the detector design, then the large amount of remaining $\Upsilon$ physics is readily accessible at an asymmetric $B$ factory. The physics we have considered requires sensitivity to branching ratios of the order of $10^{-5}$, and is thus only accessible at a high luminosity $e^{+} e^{-}$machine (independent of whether it is a symmetric or an asymmetric machine). In fact, the majority of this physics will remain untapped until such luminosities are achieved and a detector with superior photon energy resolution is employed. It offers a unique opportunity to carry out fundamental tests of $\mathrm{QCD}$, deepen our understanding of potential models, and conduct searches for new physics. 


\section{REFERENCES}

1. J. Lee-Franzini, D.B. Lichtenberg and E. Predazzi, Proceedings of the Workshop on Heavy-Quark Factory and Nuclear Physics Facility with Superconducting Linacs, ed. by E. De Sanctis, M. Greco, M. Piccolo and S. Tazzari (Editrice Compositori, Bologna, 1988) p.263.

2. U.Volland, PSI $B$ Factory Proposal, PR-88-09 (1988).

3. K. Berkelman, Rep. Prog. Phys. 49, 1 (1986).

4. W. Kwong, J.L. Rosner and C. Quigg, Ann. Rev. Nucl. and Part. Science 37, 325 (1987).

5. J. Lee-Franzini, Proceedings of the 1987 International Symposium on Lepton and Photon Interactions at High Energies, ed. by W. Bartel and R. Ruckl, Nucl. Phys. (Proc. Suppl.) B3, 139 (1988).

6. P.M. Tuts, Proceedings of the 1989 International Symposium on Heavy Quark Physics, ed. by P.S. Drell and D.L. Rubin (AIP, New York, 1989) in press.

7. T. Kaarsberg et al. (CUSB), Phys. Rev. D35, 2265 (1987); T. Kaarsberg et al. (CUSB), Phys. Rev. Lett. 62, 2077 (1989).

8. W.-Y. Chen et al. (CLEO), CLEO preprint CLEO 89-1 (1989).

9. H. Albrecht et al. (ARGUS), Z. Phys, C35, 283 (1987).

10. E. Eichten and F. Feinberg, Phys. Rev. D23, 2724 (1981).

11. J.L. Rosner, in Experimental Meson Spectroscopy-1983, ed. by S.J. Lindenbaum. AIP, New York, (1984) 461.

12. P. Moxhay and J. L. Rosner, Phys. Rev. D28, 1132 (1983).

13. R. McClary and N. Byers, Phys. Rev. D28, 1692 (1983).

14. S.N. Gupta, S.F. Radford and W.W. Repko, Phys. Rev. D30, 2425 (1984).

15. Y.P. Kuang and T.M. Yan, Phys. Rev. D24, 2871 (1981), and references therein.

16. I.C. Brock et al, CLEO preprint, CBX-88-22 (1988). 
17. P. Franzini et al., Phys. Rev. D35, 2883 (1987).

18. See for example the references in P.M. Tuts, Proceedings of the Int. Symp. on Lepton and Photon Interactions at High Energies, ed. D.G. Cassel and D.L. Kreinick (Cornell, 1983) p.284.

19.

20. Y-P. Kuang, S.F. Tuan and T-M. Yan, Phys. Rev. D37, 1210 (1988).

21. M.B. Voloshin, Sov. J. Nucl. Phys. 43, 1011 (1986).

22. W. Kwong and J.L. Rosner, Phys. Rev. D28, 279 (1988).

23. A. Martin, CERN preprint, CERN-TH.4972/88.

24. G. Belanger and P. Moxhay, Phys. Lett. B199, 575 (1987).

25. P. Moxhay, Phys. Rev. D37, 2557 (1988).

26. M.B. Voloshin, Yad. Fiz. 36, 247 (1982) Sov. J. Nucl. Phys. 36, 143 (1982).

27. J. Lee-Franzini, in Proc. 1987 Int. Symp. on Lepton and Photon interactions at high energies, DESY, Hamburg, eds. D. Haidt and R. Ruckl, Nucl. Phys. (Proc. Suppl. ) B3,139 (1988).

28. C.O. Dib, F.J. Gilman, and P.J. Franzini, Phys. Rev. D37,735 (1 988).

29. A. Martin, CERN Preprint, CERN-TH.4972/88.

30. R. Barbieri, R. Gatto and R. Kögler, Phys. Lett. B60, 183 (1976); R. Barbieri, R. Gatto and E. Remiddi, Phys. Lett. B61, 465 (1976).

31. R. Barbieri, M. Caffo, R. Gatto and E. Remiddi, Phys. Lett. B95, 93 (1980).

32. J. Fllis et al., Phys. Lett., B158, 417 (1985).

33. F. Wilczek, Phys. Rev. Lett. 40, 220 (1978).

34. S. Weinberg,Phys. Rev. Lett. 40, 223 (1978).

35. M.I. Vysotsky, Phys. Lett. B97, 159 (1980); J. Ellis et al., ibid, B158, 417 (1985); P. Nason, ibid, B175, 223 (1986). 
36. M. Drees and K. Hikasa, preprint CERN-TH.5393/89 and KEK-TH-224 (1989).

37. J. C. Pati and H. Stremnitzer, Phys. Rev. Lett. 56 (1986), 2152.

38. H. Stremnitzer and J. C. Pati, preprint Maryland Physics \# 87-135, Vienna \# UWPhTh-1986-35.

39. J. C. Pati, Phys. Rev. D30(1984), 1144.

40. Particle Data Group, Phys. Lett. 204B(1988), 1.

41. P. Franzini, Proceedings of the Workshop on HeavyQuark Factory and Nuclear Physics Facility with Superconducting Linacs, ed. by E. De Sanctis, M. Greco, M. Piccolo and S. Tazzari (Editrice Compositori, Bologna, 1988) p.665.

42. B. Borgia, F. Ferroni and E. Longo, Proceedings of the Workshop on Heavy-Quark Factory and Nuclear Physics Facility with Superconducting Linacs, ed. by E. De Sanctis, M. Greco, M. Piccolo and S. Tazzari (Editrice Compositori, Bologna, 1988) p.637. 


\section{Tau Physics at a $B$ Factory}

\subsection{INTRODUCTION}

7 he $\tau$ lepton was discovered in 1975 at the SPEAR $e^{+} e^{-}$ 1 storage ring at SLAC . The initial investigation of its properties at SPEAR and DORIS I established it as a third generation lepton and yielded first measurements of its major decay modes. When the higher energy storage rings PETRA and PEP came into operation, the situation improved considerably because increased samples of $\tau$ 's could be obtained that were relatively background free. Thus it became possible to study the semi-hadronic decays of the $\tau$ in much more detail. In addition, the known decay channels were remeasured with accuracies of a few percent typically. More recently, even larger data samples, taken at CESR and DORIS II, became available, adding to our knowledge about the $\tau$ lepton and its associated neutrino $\nu_{\tau}$. There have been several recent reviews. ${ }^{2}$ Present event samples are about $3.5 \times 10^{4}$ events maximum for each of the PEP/PETRA experiments, and about $3 \times 10^{5}$ events for each of the CESR/DORIS II experiments that operate in the region of the $\Upsilon(4 \mathrm{~S})$.

All existing measurements agree with the $\tau$ being a spin$1 / 2$ pointlike particle of unit charge with a $V-A$ weak interaction and no strong interaction. Except for phenomena related to its higher mass, no significant differences between the $\tau$ and the other charged leptons, electron and muon, have yet been found.

Compared to the precision reached in the study of other fundamental particles, such as the muon, research in $\tau$ physics still has a long way to go. There are many topics of interest to pursue in $\tau$ physics, where very large event numbers collected by a single experiment will be important. Some of these involve the $\tau$ itself, e.g. lifetime, branching ratios, rare decays. Others involve using the $\tau$ as a probe for other physics, e.g. $\nu_{\tau}$ mass, $\tau-\mu-e$ universality, weak charged currents, hadron spectroscopy, physics beyond the Standard Model.

The new generation of $e^{+} e^{-}$experiments will certainly improve matter, but statistics will continue to dominate the precision of many measurements performed over the next few 
The $B$ factory promises to produce by far the largest obtainable $\tau$ sample years, and will also limit the reduction of systematic errors, due to lack of sufficient data to exhaustively study systematic effects. Projected $\tau$ pair yields of various machines are $9 \times 10^{4}$ /year for LEP, $\sim 10^{5} /$ year for BEPC, $1.6 \times 10^{5} /$ year for HiLum PEP, and $1.5 \times 10^{7}$ after five years for CESR ${ }^{*}$. The $30 \mathrm{fb}^{-1}$ initial sample at the $B$ factory yields $3 \times 10^{7} \tau$ pairs, the $300 \mathrm{fb}^{-1}$ sample, $3 \times 10^{8}$. These samples will dominate the field even if the other experiments have been accumulating data for a number of years before the $B$ factory turns on. It should also be borne in mind that several of the features we envision for a new detector, viz. the low-mass drift chamber and ring-imaging Cerenkov counter, provide substantially improved capability for measurements such as $m_{\nu_{\tau}}$ limits and the Cabibbo angle determination.

We present a representative selection of $\tau$ measurements at a $B$ factory. Our discussion is based primarily on a sample of $3 \times 10^{7} \tau$ pairs, although in some cases we quote results for the full data sample. In our calculations we use the following $\tau$ decay branching ratios: $B_{1}=0.866, B_{3}=0.133$, $B_{e}=B_{\mu}=0.18, B_{\pi}=0.108, B_{3 \pi}=0.064, B_{K}=0.007$. Particle identification is done with a (normalized) likelihood that makes use of the information from the CRID, from the electromagnetic calorimeter, and from the muon filter.

\subsection{Limits on the TaU NeUtrino Mass}

The question of non-zero neutrino masses is now of considerable interest because of theoretical ideas such as GUT's, neutrino oscillations, and dark matter. In the Standard Model there is no fundamental principle requiring a vanishing mass for the neutrinos. On the contrary, many extensions of the Standard Model predict non-zero neutrino masses and a mass hierarchy among different neutrino generations, as is the case in the charged lepton sector. Whereas fairly stringent mass limits exist for the electron and muon neutrino ${ }^{3}\left(m_{\nu_{e}}<18 \mathrm{eV}\right.$

\footnotetext{
* In calculating these numbers we assumed: 1 year $=10^{7} \mathrm{sec}, \mathrm{L}_{\mathrm{LEP}}=$ $6 \times 10^{30} \mathrm{~cm}^{-2} \mathrm{sec}^{-1}, \sigma_{\tau \tau}(\sqrt{s}=91.2 \mathrm{GeV})=1.45 \mathrm{nb}, \mathrm{L}_{\mathrm{BEPC}}=$ $10^{31} \mathrm{~cm}^{-2} \mathrm{sec}^{-1}, \sigma_{\tau \tau(\gamma)}(\sqrt{s}=3.568-4.2 \mathrm{GeV})=0-3 \mathrm{nb}, \mathrm{L}_{\mathrm{PEP}}=$ $10^{32} \mathrm{~cm}^{-2} \mathrm{sec}^{-1}, \sigma_{\tau \tau(\gamma)}(\sqrt{s}=27 \mathrm{GeV})=0.156 \mathrm{nb}$, and $\mathrm{L}_{\mathrm{CESR}}=1-$ $5 \times 10^{32} \mathrm{~cm}^{-2} \mathrm{sec}^{-1}, \sigma_{\tau r(\gamma)}(\sqrt{s}=10.58 \mathrm{GeV})=0.91 \mathrm{nb}$.
} 
at $95 \%$ C.L. and $m_{\nu_{\mu}}<250 \mathrm{keV}$ at $90 \%$ C.L.), $m_{\nu_{r}}$ is limited only to $<35 \mathrm{MeV}$ at $95 \%$ C.L. 4

The most sensitive technique to obtain a limit on $m_{\nu_{\tau}}$ is based on studying the endpoint of the hadronic invariant mass distribution, $m_{\text {had }}^{\max }$, in $\tau$ decays into high-mass final states, such as ${ }^{*} \tau^{-} \rightarrow \nu_{\tau} \pi^{-} \pi^{+} \pi^{-} \pi^{+} \pi^{-}$and $\tau^{-} \rightarrow \nu_{\tau} K^{-} K^{ \pm} \pi^{\mp}$. The endpoint of this distribution is related to $m_{\nu_{\tau}}$ by

$$
m_{\nu_{\tau}}=m_{\tau}-m_{\text {had }}^{\max } .
$$

Neglecting for now systematic uncertainties, the $m_{\nu_{r}}$ limit that can be obtained is governed by the detector resolution for $m_{h a d}$ and by the number of events observed in the mass range close to the endpoint, $N_{o b s}$.

The projected $N_{o b s}$ depends crucially on the assumed intermediate resonance structure in the hadronic system. Existing data ${ }^{4}$ seem to support a $\rho \rho \pi$ substructure in the $5 \pi$ final state. For the $K K \pi$ final state we make use of a chiral perturbation model (see References 5 and 6 and references therein) that takes into account all accessible vector and axialvector resonances $\left(e . g . \rho^{\prime}(1700), a_{1}(1270)\right)$ in the intermediate state. The model predicts a branching ratio of $0.4 \%$, in agreement with the present experimental value of $\left(0.22_{-0.11}^{+0.17}\right) \%^{3}$.

Whereas $N_{o b s}$ is quite model-dependent, the shape of the hadronic invariant mass distribution, $\mathrm{d} \Gamma / \mathrm{d} m_{\text {had }}$, is not. It is rather dominated by the so-called weak matrix element $\omega$ and by a kinematical factor $\lambda$ !

$$
\mathrm{d} \Gamma / \mathrm{d} m_{h a d} \propto m_{h a d} \omega\left(m_{\tau}, m_{h a d}, m_{\nu_{\tau}}\right) \lambda^{1 / 2}\left(m_{\tau}, m_{h a d}, m_{\nu_{\tau}}\right),
$$

where

$$
\begin{aligned}
\omega\left(m_{\tau}, m_{\text {had }}, m_{\boldsymbol{\nu}_{\tau}}\right)= & \left(m_{\tau}^{2}-m_{\text {had }}^{2}\right)\left(m_{\tau}^{2}+m_{\text {had }}^{2}\right)- \\
& 2 m_{\boldsymbol{\nu}_{\tau}}^{2}\left(2 m_{\tau}^{2}-m_{\text {had }}^{2}-m_{\boldsymbol{\nu}_{\tau}}^{2}\right)
\end{aligned}
$$

and

$$
\begin{aligned}
\lambda\left(m_{\tau}, m_{h a d}, m_{\nu_{\tau}}\right)= & {\left[m_{\tau}^{2}-\left(m_{h a d}+m_{\nu_{\tau}}\right)^{2}\right] \times } \\
& {\left[m_{\tau}^{2}-\left(m_{h a d}-m_{\nu_{\tau}}\right)^{2}\right] . }
\end{aligned}
$$

* Throughout the text $\tau^{-}$is used for both charge states of the $\tau$.

The limit on $m_{\nu_{\tau}}$ is derived from the endpoint of the hadronic invariant mass distribution 
- Decay channel $\tau \rightarrow \nu_{\tau} 5 \pi$

We have generated events of the type

$$
\begin{gathered}
\tau_{1} \rightarrow \nu_{\tau} \pi^{-} \rho^{\circ} \rho^{\circ}, \rho^{\circ} \rightarrow \pi^{+} \pi^{-} \\
\tau_{2} \rightarrow \nu_{\tau} e \nu_{e}, \nu_{\tau} \mu \nu_{\mu} .
\end{gathered}
$$

Given $3 \times 10^{7} \tau$ pairs and $B_{5 \pi}=5.6 \times 10^{-4}{ }^{3}$, we expect to detect $5200 l-5 \pi$ events $\left(\epsilon_{l 5 \pi}=0.43\right)$, of which 910 are found in the mass region $m_{5 \pi}>1.7 \mathrm{GeV}$. (We find no indication of background from $100000 q \bar{q}$ events and $80000 c \bar{c}$ events.)

- Decay channel $\tau \rightarrow \nu_{\tau} K K \pi$

We have generated events of the type

$$
\begin{gathered}
\tau_{1} \rightarrow \nu_{\tau} K^{-} K^{ \pm} \pi^{\mp} \\
\tau_{2} \rightarrow \nu_{\tau} e \nu_{e}, \nu_{\tau} \mu \nu_{\mu} .
\end{gathered}
$$

Given $3 \times 10^{7} \tau$ pairs and $B_{K K \pi}=0.22 \%$, we expect to detect $23800 l-K K \pi$ events $\left(\epsilon_{l 5 \pi}=0.5\right)$, with 320 events above $m_{K K \pi}=1.7 \mathrm{GeV}$. (Again we find no indication of background from $100000 q \bar{q}$ events and $80000 c \bar{c}$ events. Background from $\tau_{1} \rightarrow \pi \pi \pi$ and $\tau_{1} \rightarrow K \pi \pi$ has been found to contribute zero and two events, respectively. None of them fell into the endpoint region.)

To estimate the $m_{\nu_{\tau}}$ limit that can be obtained from both channels, the following procedure was applied:

- Generate $\tau_{1} \rightarrow \nu_{\tau} \pi \rho \rho, \nu_{\tau} K K \pi$ events with $m_{\nu_{\tau}}=0,1,2,3,4,6,8,10,15,20 \mathrm{MeV}$.

- Fit the invariant mass distributions above $1.7 \mathrm{GeV}$ to get $\mathrm{d} \Gamma / \mathrm{d} m_{\text {had }}$.

- Calculate the likelihood that the distributions from two appropriately sized test samples of $\tau_{1} \rightarrow \nu_{\tau} \pi \rho \rho, \nu_{\tau} K K \pi$ events (generated with $m_{\nu_{\tau}}=0 \mathrm{MeV}$ ) agree with any of the $m_{\nu_{\tau}}$ hypotheses stated above (taking into account the Gaussian mass resolutions $G_{m}$ with $\sigma\left(m_{5 \pi}\right)=$ 
$4.5 \mathrm{MeV}$ and $\left.\sigma\left(m_{K K \pi}\right)=3.5 \mathrm{MeV}\right)$ :

$$
\mathcal{L}=\prod_{\text {channels }} \prod_{\text {events }} \int \mathrm{d} m_{\text {had }} \frac{\mathrm{d} \Gamma / \mathrm{d} m_{\text {had }}}{\Gamma_{\text {tot }}} \times G_{m}
$$

- Plot $\mathcal{L}$ vs $m_{\nu_{\tau}}$ and integrate up to $95 \%$ of its area to obtain a $95 \%$ C.L. upper limit on $m_{\nu_{r}}$.

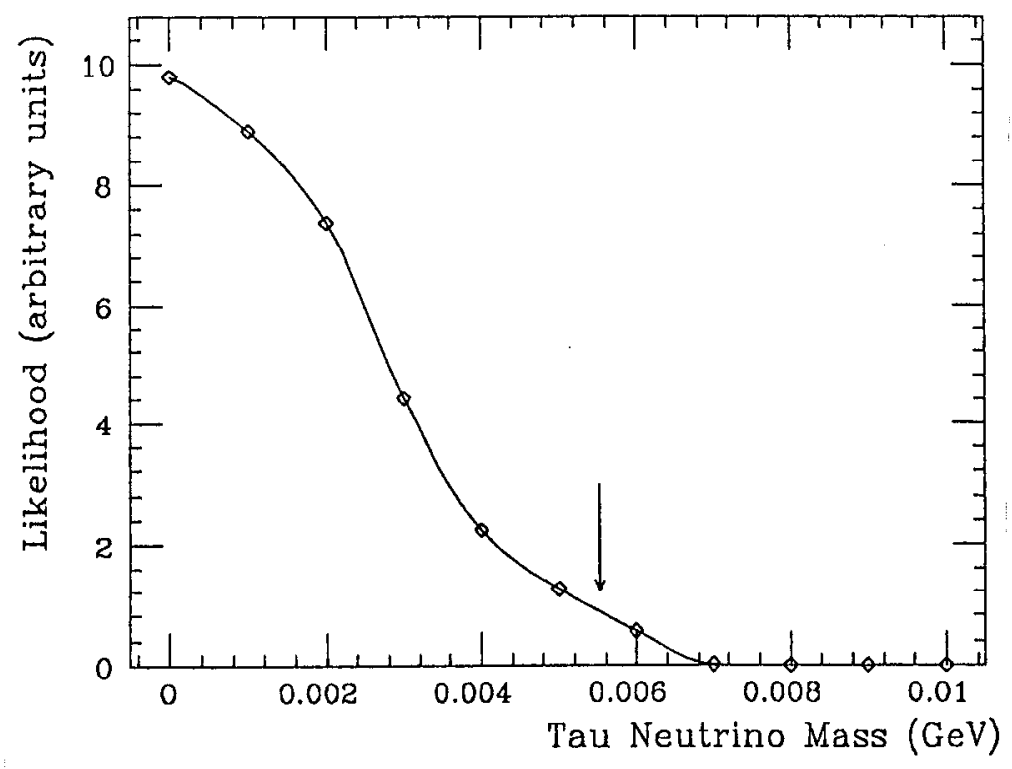

Figure 8.1 shows that, given the assumptions stated above, we can reach a limit of

$$
m_{\nu_{\tau}}<5.5 \mathrm{MeV} \text { at } 95 \% \text { C.L. }
$$

with the initial data sample. (The initial 95\% C.L. limits for each individual channel are $m_{\nu_{\tau}}<7 \mathrm{MeV}$ for $5 \pi$ and $m_{\nu_{\tau}}<$ $9 \mathrm{MeV}$ for $K K \pi$.)

For the full course of the experiment $\left(3 \times 10^{8}\right.$ produced $\tau$ pairs after $\sim$ five years of running), the projected ultimate $m_{\nu_{\tau}}$ limit is

$$
m_{\nu_{\tau}}<1.7 \mathrm{MeV} \text { at } 95 \% \text { C.L. }
$$

Clearly the limit will be somewhat degraded by systematic effects like the uncertainty of $m_{\tau}$ and of the measured $m_{\text {had }}$. We assume that $m_{\tau}$ will be measured by BEPC to
Figure 8.1 Likelihood $\mathcal{L}$ vs $m_{\nu_{r}}$. The arrow indicates $95 \%$ of the total integral.
A limit of $m_{\nu_{\tau}}<1.7 \mathrm{MeV}$ at 95\% C.L. can be obtained with the $300 \mathrm{fb}^{-1}$ sample 
$\tau$ decay allows a very clean way to measure the Cabibbo angle

within $1 \mathrm{MeV}$, and that the hadronic mass scale in the experiment is found to $1 \mathrm{MeV}$ by studying, e.g., inclusive $K_{S}^{\circ}$. - These systematic errors will worsen the one-year upper limit on $m_{\boldsymbol{\nu}_{\tau}}$ by $0.7 \mathrm{MeV}$ and the five-year upper limit by $1.5 \mathrm{MeV}$.

\subsection{Measurement of the Cabibbo Angle}

The Cabibbo angle, as defined originally by N. Cabibbo, is given by the ratio of the muonic decay widths of $K$ and $\pi$ :

$$
\frac{\Gamma\left(K \rightarrow \nu_{\mu} \mu\right)}{\Gamma\left(\pi \rightarrow \nu_{\mu} \mu\right)}=\tan ^{2} \theta_{C} \frac{m_{K}\left(1-m_{\mu}^{2} / m_{K}^{2}\right)^{2}}{m_{\pi}\left(1-m_{\mu}^{2} / m_{\pi}^{2}\right)^{2}} .
$$

The Cabibbo angle can also be measured in $\tau$ decay, the only difference (apart from radiative corrections) being that the $\nu_{\mu}-W-\mu$ vertex is replaced by the $\nu_{\tau}-W-\tau$ vertex:

$$
\frac{\Gamma\left(\tau \rightarrow \nu_{\tau} K\right)}{\Gamma\left(\tau \rightarrow \nu_{\tau} \pi\right)}=\tan ^{2} \theta_{C} \frac{\left(1-m_{K}^{2} / m_{\tau}^{2}\right)^{2}}{\left(1-m_{\pi}^{2} / m_{\tau}^{2}\right)^{2}} .
$$

The ratio of the partial widths equals the ratio of the $\tau$ branching ratios into $K$ and $\pi, B_{K} / B_{\pi}$. At present, this ratio is known only to a precision of about $25 \%^{3}$. A dedicated measurement at the $B$ factory can improve the precision by a factor of 40 by measuring the number of events where

$$
\begin{gathered}
\tau_{1} \rightarrow \nu_{\tau} K \text { or } \nu_{\tau} \pi \\
\tau_{2} \rightarrow \nu_{\tau} e \nu_{e}, \nu_{\tau} \mu \nu_{\mu} .
\end{gathered}
$$

Then $B_{K} / B_{\pi}$ is given by

$$
\frac{B_{K}}{B_{\pi}}=\frac{N_{l K} / \epsilon_{l K}}{N_{l \pi} / \epsilon l \pi}=\frac{2 B_{l} B_{K} N_{\tau \tau}}{2 B_{l} B_{\pi} N_{\tau \tau}},
$$

where $B_{l}$ and $N_{\tau r}$ cancel.

Our studies show that we can detect $1.5 \times 10^{5} \times\left(\epsilon_{l K}=\right.$ $0.34)=5.2 \times 10^{4} l-K$ events and $2.3 \times 10^{6} \times\left(\epsilon_{l \pi}=0.42\right)=$ $9.7 \times 10^{5} l-\pi$ events from $3 \times 10^{7}$ produced $\tau$ pairs, yielding a statistical error on $B_{K} / B_{\pi}$ of $0.45 \%$. (We have assumed equal efficiency for $l=e$ and $l=\mu$.) 
To estimate the background, which is mainly due to particle misidentification, we checked other $\tau$ decay channels as well as $q \bar{q}$ events (see Table 8.1 ).

Assuming that the Monte Carlo correction for this background is accurate to within $10 \%$ yields a systematic error of $0.3 \%$. The difference in efficiency, which is mainly due to a momentum cut to keep particle identification efficient, contributes another $0.3 \%$. Thus we expect to measure $B_{K} / B_{\pi}$ to a precision of $0.6 \%$, where statistical and systematic errors have been combined in quadrature. Assuming that the $\tau$ mass is measured by then to $1 \mathrm{MeV}$, this yields a $0.6 \%$ measurement of the Cabibbo angle in $\tau$ decay.

Table 8.1 Background contributions to $e-\pi$ and $e-K$.

\begin{tabular}{|c|c|c|}
\hline \multicolumn{3}{|c|}{ Background contribution (\%) } \\
\hline From & To $e-\pi$ & To $e-K$ \\
\hline$e-\mu$ & 2.6 & 0.18 \\
\hline$e-\pi$ & - & 0.42 \\
\hline$e-K$ & 0.013 & - \\
\hline$e-e$ & 0.023 & - \\
\hline$q \bar{q}$ & 0.7 & 0.14 \\
\hline
\end{tabular}

\subsection{Second Class Currents}

The weak charged current can be classified into first and second-class currents according to its transformation under G parity (see Reference 8 and references therein) as shown in Table 8.2.
The $B$ factory can make a $0.6 \%$ measurement of the Cabibbo angle in $\tau$ decay with the 30 $\mathrm{fb}^{-1}$ sample 
The expected branching fraction for the second-class current decay $\tau^{-} \rightarrow \nu_{\tau} \pi^{-} \eta$ is $1.5 \times 10^{-5}$

We expect 170 signal events in $90 \mathrm{fb}^{-1}$
Table 8.2 Classifications of the weak charged current.

\begin{tabular}{|c|c|c|}
\hline & vector current & axial current \\
\hline first class & $\mathrm{G}=+1$ & $\mathrm{G}=-1$ \\
\hline second class & $\mathrm{G}=-1$ & $\mathrm{G}=+1$ \\
\hline
\end{tabular}

An example of a $\tau$ decay that proceeds through a secondclass current is $\tau^{-} \rightarrow \nu_{\tau} \pi^{-} \eta$. Since the hadronic final state consists of two pseudoscalars, the decay must proceed through the vector current, which is characterized by $\mathrm{G}=+1$. However, the $\pi \eta$ system has $\mathrm{G}=-1$. Thus this process involves a second-class current.

Second-class currents are strongly suppressed in the Standard Model since they involve some symmetry breaking ${ }^{8}$ at the $W q \bar{q}$ vertex (e.g., due to the $\mathrm{u}-\mathrm{d}$ mass difference or radiative corrections), or symmetry breaking in the hadronization process, or another non-standard decay mechanism (e.g., due to a scalar particle like the charged Higgs boson).

From isospin-violating effects alone, which are suppressed by the square of the $\mathrm{u}-\mathrm{d}$ mass difference, the predicted branching ratio ${ }^{9}$ for $\tau^{-} \rightarrow \nu_{\tau} \pi^{-} \eta$ is $1.5 \times 10^{-5}$. Measuring this branching ratio provides an interesting test of the Standard Model and offers the potential for finding new physics beyond it.

We have studied the possibility of measuring the decay channel $\tau^{-} \rightarrow \nu_{\tau} \pi^{-} \eta$, where the $\eta$ is observed via its decay into two photons $(B(\eta \rightarrow \gamma \gamma)=0.389)^{3}$. (The channel $\tau^{-} \rightarrow$ $\nu_{\tau} \pi^{-} \eta, \eta \rightarrow \pi^{+} \pi^{-} \pi^{\circ}$ suffers from a huge background from the unsuppressed decay $\tau^{-} \rightarrow \nu_{\tau} \pi^{-} \pi^{+} \pi^{-} \pi^{\circ}$, which has a branching ratio of $\left.4.4 \times 10^{-2}\right){ }^{3}$. We select events with

$$
\begin{gathered}
\tau_{1} \rightarrow \nu_{\tau} \pi^{-} \eta, \eta \rightarrow \gamma \gamma \\
\tau_{2} \rightarrow \nu_{\tau} e \nu_{e}, \nu_{\tau} \mu \nu_{\mu}, \nu_{\tau} \pi, \nu_{\tau} K .
\end{gathered}
$$

With $9 \times 10^{7}$ produced $\tau$ pairs and the assumed branching ratio, we expect to detect 170 signal events $\left(\epsilon_{\pi \eta}=0.34\right)$ over a large background from the following processes: 
- $\tau_{1} \rightarrow \nu_{\tau} \rho^{-} \rightarrow \nu_{\tau} \pi^{-} \pi^{\circ}, \pi^{\circ} \rightarrow \gamma \gamma\left(B\left(\tau_{1}\right)=2.23 \times\right.$ $\left.10^{-1}\right)^{3}$, where one of the photons is lost, but an initial state radiation photon is detected instead. (This process contributes about $1 / 3$ of the background.)

- $\tau_{1} \rightarrow \nu_{\tau} \pi^{-} \pi^{\circ} \pi^{\circ}, \pi^{\circ} \rightarrow \gamma \gamma\left(B\left(\tau_{1}\right)=8.5 \times 10^{-2}\right),{ }^{10}$ where two photons are lost. (This process contributes about $2 / 3$ of the background.)

Background from $\tau_{1} \rightarrow \nu_{\tau} \pi^{-} \pi^{\circ} \pi^{\circ} \pi^{\circ}\left(B\left(\tau_{1}\right)=1.0 \times 10^{-2}\right),{ }^{11}$ $\tau_{1} \rightarrow \nu_{\tau} \pi^{-} \pi^{\circ} \eta\left(B\left(\tau_{1}\right)=1.5 \times 10^{-3}\right),{ }^{12}$ and $q \bar{q}$ were found to be small.

Figure 8.2(a) shows the $\gamma \gamma$ invariant mass spectrum expected with $90 \mathrm{fb}^{-1}$. The signal at the $\eta$ mass has a $4 \sigma$ significance. Were the $\tau \rightarrow \nu_{\tau} \pi^{-} \eta$ branching fraction to be $3 \times 10^{-5}$, we would find a $5 \sigma$ signal with only $30 \mathrm{fb}^{-1}$ (see Figure 8.2(b)).

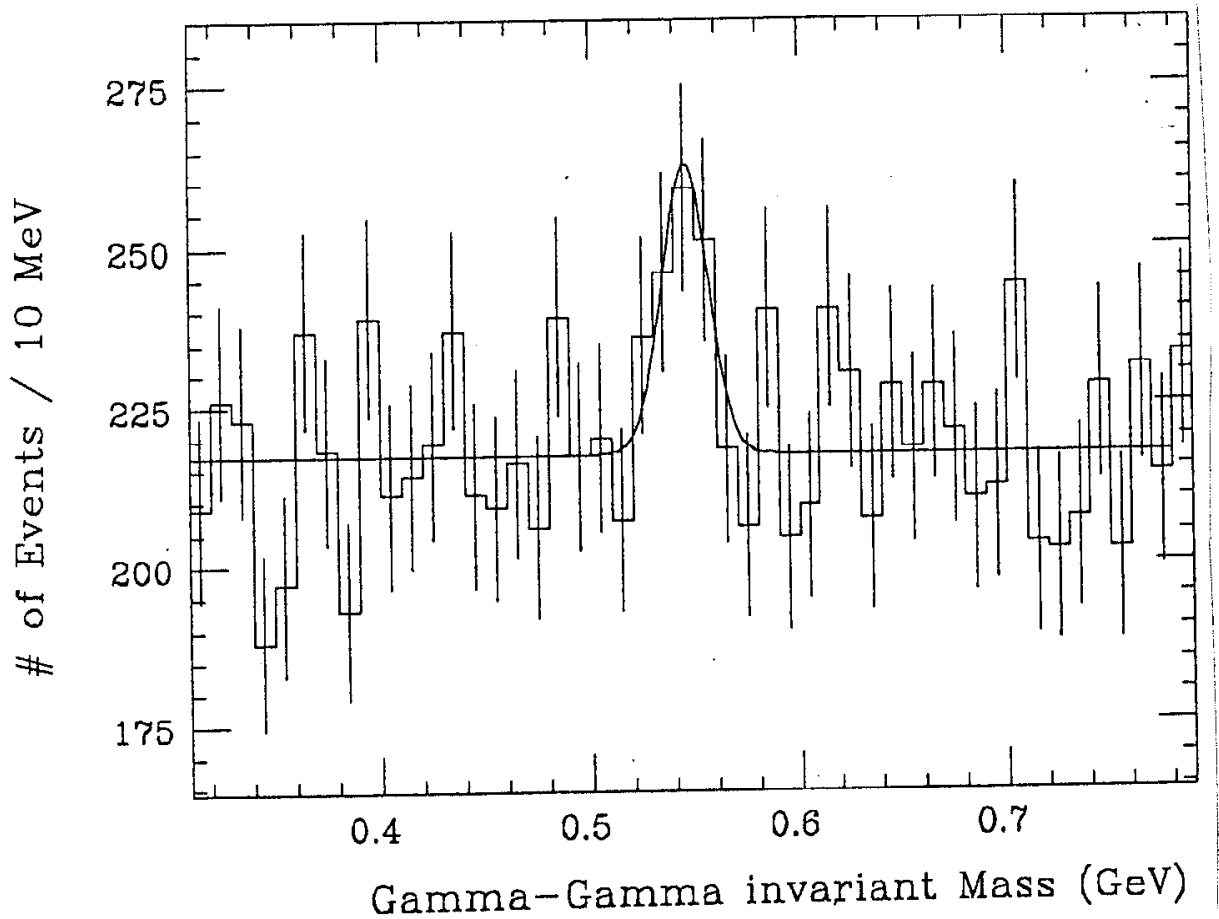

With $90 \mathrm{fb}^{-1}$, a limit of $<3 \times 10^{-5}$ can be obtained
Figure 8.2(a) $\gamma \gamma$ invariant mass spectrum for $B_{\pi \eta}=1.5 \times$ $10^{-5}$ in the $90 \mathrm{fb}^{-1}$ sample 
Figure 8.2(b) $\gamma \gamma$ invariant mass spectrum for $B_{\pi \eta}=3 \times$ $10^{-5}$ in the $30 \mathrm{fb}^{-1}$ sample
The high mass of the $\tau$ enhances sensitivity to new particles

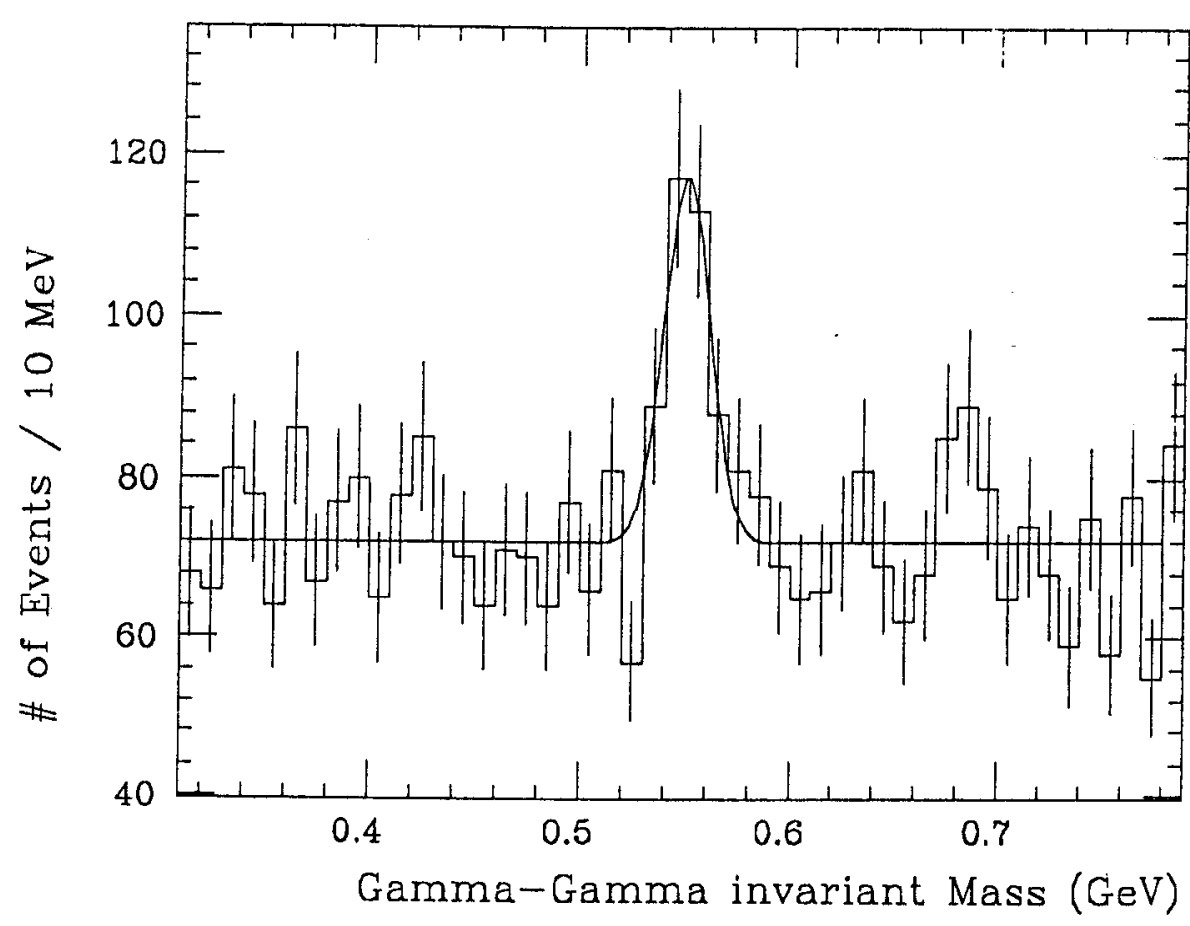

\subsection{RARE DECAYS}

The large sample of $\tau$ pairs produced at a $B$ factory offers a unique possibility to look for $\tau$ decays forbidden in the Standard Model. As an example, we have investigated the possibility for detecting the neutrinoless decay $\tau \rightarrow \mu \mu \mu$, which violates lepton flavor and could be mediated by, e.g., a charged Higgs boson.

All existing data are consistent with the conservation of lepton flavor and lepton number. However, there is no obvious theoretical reason for this within the Standard Model. In fact, many extensions predict such violations at some level and experimental searches for them probe these models up to mass scales of a few $\mathrm{TeV}$. The most stringent limits to date come from studies of $\mu, \pi$ and $K$ decays. (For example ${ }^{3}$ $B(\mu \rightarrow e e e)<1.0 \times 10^{-13}$ whereas $B(\tau \rightarrow \mu \mu \mu)<2.9 \times 10^{-5}$, both limits at $90 \%$ C.L.) However, very different violation patterns can emerge, due to the mass differences between the generations. Generally the effects of new particles are enhanced for heavier fermions, resulting in an increased competitiveness of $\tau$ decay experiments. For example, for a neutrinoless leptonic decay, mediated by a charged Higgs boson, 
one estimates $^{13}$

$$
\frac{B(\tau \rightarrow \mu \mu \mu)}{B(\mu \rightarrow e e e)} \simeq\left(\frac{m_{\tau}}{m_{e}}\right)^{2} \simeq 10^{7} .
$$

Thus a $\tau$ decay limit below $10^{-6}$ has a higher sensitivity than the present $\mu$ decay limit.

We have studied the sensitivity that can be reached in a search for the decay $\tau \rightarrow \mu \mu \mu$. For a decay branching ratio of $10^{-7}$ and a $\tau$ pair sample of $9 \times 10^{7}$, we expect to detect four events with

$$
\begin{gathered}
\tau_{1} \rightarrow \mu \mu \mu \\
\tau_{2} \rightarrow \nu_{\tau} e \nu_{e}, \nu_{\tau} \mu \nu_{\mu}, \nu_{\tau} \pi, \nu_{\tau} K .
\end{gathered}
$$

Since the searched for decay is neutrinoless, the total invariant mass of the three muons will be clustered around the $\tau$ mass, giving the events a unique signature.

Several sources of background have been investigated:

- $\tau_{1}^{-} \rightarrow \nu_{\tau} \pi^{-} \pi^{+} \pi^{-}\left(B\left(\tau_{1}\right)=6.4 \times 10^{-2}\right)$, where all pions get misidentified as muons. We find a contribution of ten such events; however, at most one would fall into the $\tau$ mass region from $1.75 \mathrm{GeV}$ to $1.82 \mathrm{GeV}$.

- $\tau_{1}^{-} \rightarrow \nu_{\tau} \pi^{-} \rho^{0}, \rho^{0} \rightarrow \mu \mu\left(B\left(\tau_{1}\right)=6.4 \times 10^{-2} \times 6.7 \times\right.$ $\left.10^{-5}\right)^{3}$, where one pion gets misidentified as a muon. This process contributes six events. Again we expect at most one event in the $\tau$ mass region.

- $e^{+} e^{-} \rightarrow q \bar{q}$. We find that $3.6 \times 10^{4}$ events pass all cuts except the one on particle identification. Assuming conservatively that only two out of three tracks (assumed to be pions) have to be misidentified as muons $\left(\epsilon_{\text {misid }}=0.0152\right)$ results in an cxpected six background events; at most one of these is close to the $\tau$ mass.

Thus we expect to observe four signal events and three background events. Using Poisson statistics, this can be converted into an upper limit

$$
B(\tau \rightarrow \mu \mu \mu)<3 \times 10^{-7} \text { at } 95 \% \text { C.L. }
$$

Similar limits are achievable for most of the neutrinoless 
A limit of $B_{\mu \mu \mu}<3 \times 10^{-7}$ at $95 \%$ C.L. has a sensitivity comparable to muon decay limits $\tau$ decays predicted in models (see Reference 13 and references therein) involving leptoquarks $\left(\tau \rightarrow \mu \pi^{\circ}, \mu \rho^{\circ}\right)$, supersymmetry $(\tau \rightarrow \mu \gamma)$, and compositeness $(\tau \rightarrow \mu \phi)$.

\subsection{Structure of the $\tau-W-\nu_{\tau}$ Vertex}

The exact form of the $\tau$ coupling to the weak charged current is far from being sufficiently constrained by existing data. The most general 4-fermion interaction Hamiltonian ${ }^{14}$ contains ten complex coupling constants, with one phase leading to 19 real parameters to be determined experimentally. It has been shown ${ }^{15}$ that the structure of the weak charged current can be determined completely by measuring the following observables:

(a) Lifetime $\tau$

(b) Decay asymmetry $\xi$ and $\delta$ of the daughter lepton ( $e$ or $\mu$ ) relative to the spin of the mother lepton

(c) Polarization $\xi^{\prime}$ of the daughter lepton

(d) Total cross scction $S$ for the inverse decay $\nu_{\tau} e \rightarrow \tau \nu_{e}$ with $\nu_{\tau}$ of known helicity.

All corresponding quantities have been measured in $\mu$ decay, leading to a lower limit for the $V-A$ interaction and upper limits for all other interactions. 15

Such a precision test of the structure of the $\tau-W-\nu_{\tau}$ vertex has hardly begun. Besides the $\tau$ lifetime, $\tau_{\tau}=(3.04 \pm 0.09) \times$ $10^{-13} \mathrm{sec}{ }^{3}$, the only decay parameter measured to date is the Michel parameter ${ }^{16} \rho: \rho=0.70 \pm 0.05^{17}$ which is consistent with the $V-A$ expectation of 0.75 . (Note that measuring the $V-A$ value for $\rho$ does not exclude phenomena like righthanded vector bosons or charged Higgs bosons, which are common to many extensions of the Standard Model.) The values of $\rho$ and of the 'low energy spectrum parameter' $\eta$ are in principle determined by the above five measurements. However, since measurement of $\xi^{\prime}$ and $S$ is hardly conceivable at present, a direct measurement of $\rho$ and $\eta$ from the lepton momentum spectrum in $\tau \rightarrow \nu_{\tau} l \nu_{l}$ adds valuable information. The determination of the strength of the interaction from the 
$\tau$ lifetime actually requires knowledge of $\eta$, since $^{14}$

$$
G_{F}^{2}=\frac{1}{\tau_{\tau}} \frac{192 \pi^{3}}{m_{\tau}^{5}} \frac{1}{1+4 \eta\left(m_{l} / m_{\tau}\right)-8\left(m_{l} / m_{\tau}\right)^{2}}
$$

The determination of the decay asymmetry parameters $\xi$ and $\delta$ requires knowledge of the $\tau$ polarization. This is possible (without polarized beams) since the spins of two $\tau$ 's produced in $e^{+} e^{-}$annihilation are strongly correlated. (The helicities of the $\tau$ 's tend to be opposite to each other as their $\beta$ 's increase.$^{18}$ )

The prospects for measuring $\rho, \eta, \xi$ and $\delta$ have been extensively studied in References 5 and 19. The authors conclude that a $B$ factory can measure the above parameters with statistical errors between a few tenths of a percent (for $\rho$ and $\delta$ ) and a few percent (for $\xi$ and $\delta$ ). (So far no estimates for systematic errors for these measurements exist.)

A determination of these parameters will probe in a model independent way the weak charged current contributions from right-handed vector bosons and from scalar and tensor bosons. This is an important step to fully establish the $V-A$ type interaction of the $\tau$.

\subsection{Measurement of $B_{e} / B_{\mu}$}

The leptonic $\tau$ decays $\tau \rightarrow \nu_{\tau} e \nu_{e}$ and $\tau \rightarrow \nu_{\tau} \mu \nu_{\mu}$ provide an ideal testing ground for our understanding of the $\tau$ lepton and its weak interaction, for two reasons: (1) The decays can be calculated rigorously, and (2) their experimental signature allows precise measurements. The decay width for $\tau \rightarrow \nu_{\tau} l \nu_{l}$ is given by ${ }^{18}$

$$
\Gamma_{l}=\frac{G_{F}^{2} m_{\tau}^{5}}{192 \pi^{3}}\left(1-8 y-8 y^{3}-y^{4}-y^{2} \ln y\right)
$$

assuming $y=m_{l} / m_{\tau}, m_{\nu_{r}}=m_{\nu_{l}}=0$ and $m_{W}=\infty$. The correction for the finite $W$ mass is $0.03 \%$ and the first order electroweak radiative corrections amount to $0.43 \%{ }^{20}$ The phase space correction due to the finite $l$ mass is $2.7 \%$. Note that according to lepton universality the same strength of
It is possible to measure $\rho, \eta$ to $\mathcal{O}(0.1 \%)$ and $\xi, \delta$ to $\mathcal{O}(1 \%)$

These measurements are important to firmly establish the $V-A$ coupling of the $\tau$

Leptonic $\tau$ decays provide an ideal testing ground for our understanding of the $\tau$ 
the interaction, $G_{F}$, was assumed for $e$ and $\mu$. (Different strengths would be caused by new particles with mass or generation dependent couplings, e.g., the charged Higgs boson.) Measuring $\Gamma_{e} / \Gamma_{\mu}=B_{e} / B_{\mu}$ to a precision of a few tenths of a percent provides a sensitive test of lepton universality and of the electroweak radiative corrections. The precision of today's measurements ${ }^{3} B_{e}=(17.8 \pm 0.4) \%$ and $B_{\mu}=(17.5 \pm 0.4) \%$, does not allow stringent enough tests.

We have studied the possibility of measuring $B_{e} / B_{\mu}$ using events of the type

$$
\begin{gathered}
\tau_{1} \rightarrow \nu_{\tau} e \nu_{e} \text { or } \nu_{\tau} \mu \nu_{\mu} \\
\tau_{2} \rightarrow \nu_{\tau} \rho .
\end{gathered}
$$

Then $B_{e} / B_{\mu}$ is given by

$$
\frac{B_{e}}{B_{\mu}}=\frac{N_{e \rho} / \epsilon_{e \rho}}{N_{\mu \rho} / \epsilon_{\mu \rho}}=\frac{2 B_{e} B_{\rho} N_{\tau \tau}}{2 B_{\mu} B_{\rho} N_{\tau \tau}},
$$

where $B_{\rho}$ and $N_{\tau \tau}$ cancel. We expect to detect $2.41 \times 10^{6} \times$ $\left(\epsilon_{e \rho}=0.316\right)=7.60 \times 10^{5} e-\rho$ events and $2.41 \times 10^{6} \times\left(\epsilon_{\mu \rho}=\right.$ $0.310)=7.47 \times 10^{5} \mu-\rho$ events, yielding a statistical error on $B_{e} / B_{\mu}$ of $0.2 \%$. Note that the efficiencies for both channels are nearly identical, the difference of $1.7 \%$ all being due to the particle identification requirements. Table 8.3 shows the estimated contaminations from other $\tau$ decays and from $q \bar{q}$ events. 
Table 8.3 Background contributions to $e-\rho$ and $\mu-\rho$.

\begin{tabular}{|c|c|c|}
\hline \multicolumn{3}{|c|}{ Background contribution (\%) } \\
\hline From & To $e-\rho$ & To $\mu-\rho$ \\
\hline$e-\rho$ & & 0.16 \\
\hline$\mu-\rho$ & 0.09 & \\
\hline$e-\rho \pi^{\circ}$ & 1.47 & 0.01 \\
\hline$\mu-\rho \pi^{\circ}$ & 0.01 & 1.39 \\
\hline$\pi-\rho$ & 0.17 & 0.45 \\
\hline$q \bar{q}$ & 0.5 & 0.5 \\
\hline
\end{tabular}

This measurement allows a

Assuming that the Monte Carlo is accurate to within $10 \%$ in correcting for this background and for the difference in the detection efficiencies, we estimate a total systematic error of $0.4 \%$. Combining statistical and systematic error in quadrature results in a total error on $B_{e} / B_{\mu}$ of $0.5 \%$. Such a precision provides a stringent test of lepton universality and is high enough to see any significant deviation from the expected electroweak radiative corrections in $\tau$ decay.

\subsection{Measurement of the Tau lifetime}

For the topology where one tau decays to $e \nu \nu$ and the other to $3 \pi^{ \pm} \nu$, there will be $4.1 \times 10^{5}$ events passing our $59 \%$ acceptance cuts. This large number of taus, combined with a silicon pixel type vertex detector and a small beam pipe, will yield an extremely precise measurement of the tau lifetime. 
Figure 8.3 Decay length distribution (laboratory frame) of $\tau \rightarrow 3 \pi^{ \pm} \nu$ events for (a) a $\tau$ lifetime of $c \tau=0$, and $(b)$ a $\tau$ lifetime of $c \tau=0.0858 \mathrm{~mm}$.
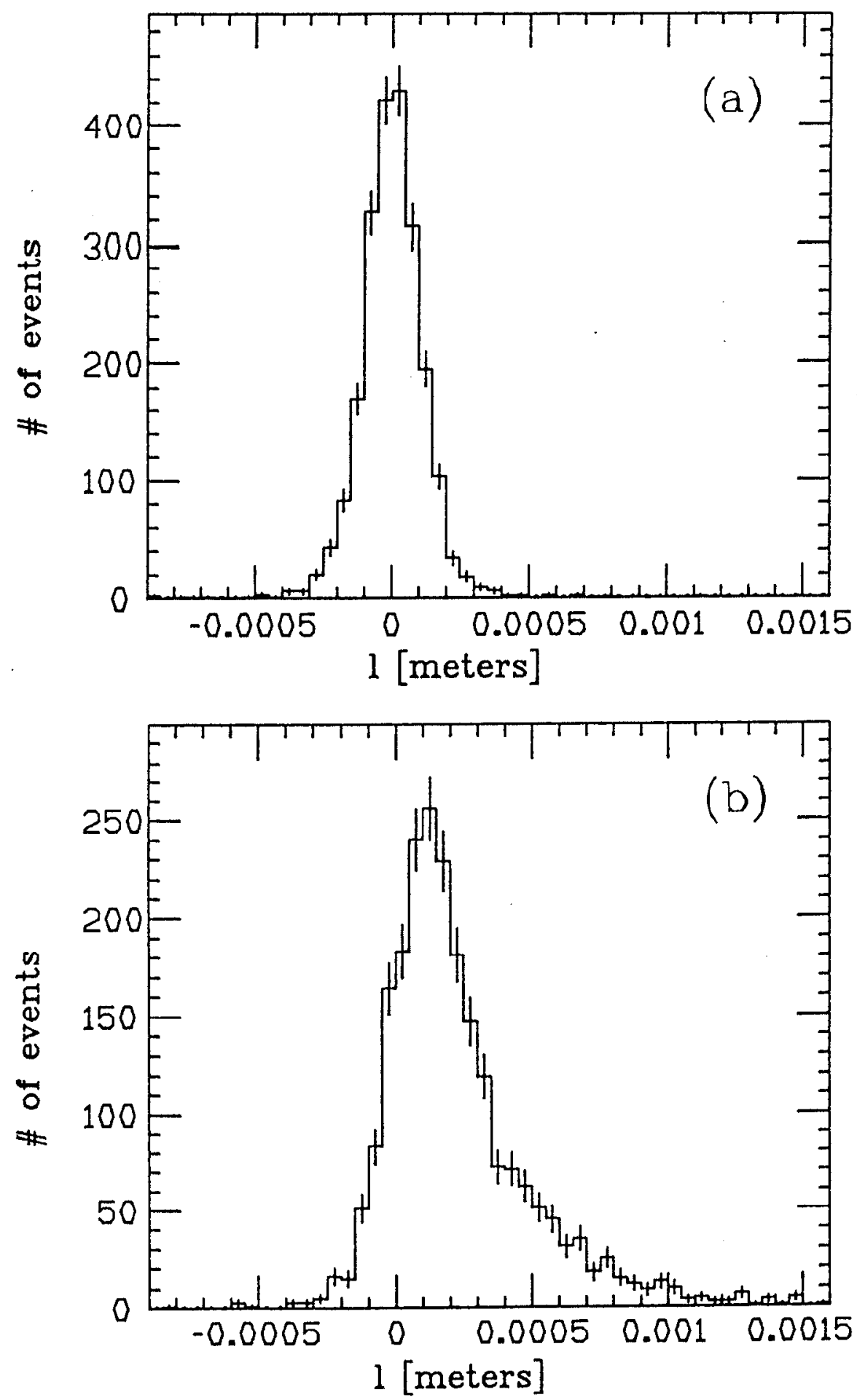

For a tau lifetime of zero and using the Mark II secondary vertex finder subroutine, Figure 8.3(a) shows the decay length distribution $(l=\beta \gamma c \tau)$ for a pure Monte Carlo sample of $\tau \rightarrow$ $3 \pi^{ \pm} \nu$ after passing through the detector simulation and vertex finding. For a tau lifetime of $2.86 \times 10^{-13} \mathrm{sec}$, Figure $8.3(\mathrm{~b})$ 
shows the same decay length distribution. The distribution is clearly asymmetric.

Using Monte Carlo events, a plot can be made of the reconstructed average decay length, $\langle l\rangle$, vs the generated tau lifetime. Assuming that this curve (very nearly a straight line) is simulated to arbitrary statistical accuracy, it can be used to read off the tau lifetime given an $\langle l\rangle \pm \Delta\langle l\rangle$ measured for the actual data. The fractional error in the tau lifetime is then just equal to the fractional error in $\langle l\rangle$. For 2207 Monte Carlo events $\tau \rightarrow 3 \pi^{ \pm} \nu$, the fractional error is $\frac{\Delta<l>}{\langle l}=2.6 \%$. Scaling this by the square root of the number of expected events (now including $\mu-3 \pi^{ \pm}$events) yields a statistical error on the tau lifetime of $\frac{\Delta\langle l\rangle}{\langle l\rangle}=0.14 \%$ for $30 \mathrm{fb}^{-1}$ of data.

For this large data sample the dominant error will be systematic. If the cuts allow too much background into the decay length distribution, the systematic error on $\langle l\rangle$ will be based on how well the $\mathrm{MC}$ models this pollution. The selection we developed has an acceptance of $59 \%$ for $e-3 \pi^{ \pm}$while letting through $<1 / 30000$ of the $q \bar{q}$ events. This translates into $1 \%$ of the events in Figure 8.3(b) being due to $q \bar{q}$. Since particle identification of the $3 \pi^{ \pm}$eliminates charged kaons and hence suppresses charm and bottom decays, we assume all the pollution events have $\langle l\rangle=0$. Then the $\langle l\rangle$ of the plot will shift by $0.5 \%$. The Monte Carlo will correct for this, but we estimate the Monte Carlo will have a $\pm 10 \%$ systematic uncertainty. Thus, we estimate a $0.05 \%$ overall systematic error on the $\tau$ lifetime from $q \bar{q}$ backgrounds. Because we are measuring $\langle l\rangle$, many potential sources of systematic error disappear to first order. For example, if the collision point of the beams moves, some decay lengths get longer and some get shorter, but $\langle l\rangle$ remains unchanged. One source of error which does not cancel out is an error in the average radius of the vertex detector. This is the main determinant of the scale of measured lengths, of what a meter of decay length means. The impact parameter of a track of a given angle is proportional to the radius of the vertex detector. Thus we estimate $\frac{\Delta\langle l\rangle}{\langle l\rangle}=\frac{\Delta R}{R}=\frac{50 \mu}{2 c m}=0.25 \%$.
A $\tau$ lifetime measurement with $0.14 \%$ statistical and $0.3 \%$ systematic error is possible with the $30 \mathrm{fb}^{-1}$ sample 


\subsection{TAU BRANChing Ratios}

Our excellent tau lifetime must be multiplied by measured branching ratios in order to get partial decay widths to compare with theoretical calculations, for example to test lepton universality at the $l-W-\nu_{l}$ vertex. To make full use of our small error on the lifetime, we want a measurement error of $\frac{\Delta B r}{B r} \simeq 0.1 \%$. This precludes the use of the (typically $2 \%$ accurate) luminosity monitor to determine the number of parent taus. Instead, one tau of the pair can be tagged, with a minimum of restrictions placed on the opposite tau so as not to enhance one branching fraction over another.

The tagged $\tau$ will be identified by its decay to $3 \pi^{ \pm} \nu$. Since $>3$ prong decays have branching ratios $<0.2 \%$, we require that the event have 4 or 6 charged prongs. Next, we boost all charged and neutral tracks to the beam-beam center of mass and divide the tracks into two hemispheres based on the thrust axis. We keep the event if in at least one hemisphere there are exactly 3 charged tracks summing to \pm 1 total charge, the tracks are identified as pions by the particle ID, and the hemisphere has no photons with $E_{\gamma}>100 \mathrm{MeV}$. Next we require that $m_{3 \pi}<m_{\tau}$, and that $\left|\cos \theta_{\tau}\right|<0.8$. The angle $\theta_{\tau}$ is between the approximate tau direction (given by the sum of the 3 pion momenta) in the lab frame and the beam axis. Finally, we require the separation of the $3 \pi$ vertex from the origin to satisfy $250 \mu<l<1500 \mu$. The remaining events are the tagged $\tau \bar{\tau}$ sample. The numbers of various tau decays in the opposite hemisphere can now be counted. Table 8.4 lists the various cuts and their effects on both $\tau \bar{\tau}$ and $q \bar{q}$ Monte Carlo events.

For $30 \mathrm{fb}^{-1}$ of data there will be approximately $6.7 \times 10^{5}$ tagged $\tau \bar{\tau}$ events. For example, we will be able to measure $B_{e}$ with a statistical error of $\frac{\Delta B r(\tau \rightarrow e \nu \nu)}{B r}=0.3 \%$. The dominant error will be systematic, one source being from $q \bar{q}$ pollution of the tagged $\tau \bar{\tau}$ sample. There will be 4 times more $q \bar{q}$ than $\tau \bar{\tau}$ because of $\mathrm{R}$ and the $\Upsilon(4 S)$ resonance, $1 / 0.124$ more because of $\operatorname{Br}\left(\tau \rightarrow 3 \pi^{ \pm} \nu\right)=0.064$ for either tau, and $\frac{0.00012}{0.18}$ times less because of the Monte Carlo-calculated acceptance of the cuts. Thus $2 \%$ of the tagged $\tau \bar{\tau}$ events are really $q \bar{q}$. If the Monte Carlo is believed to $\pm 10 \%$, then there will be a $0.2 \%$ 
systematic error on the number of $\tau$ decays seen in any particular channel due to the pollution from $q \bar{q}$ events. The Monte Carlo study is being continued to determine the additional acceptance penalties to be paid for identifying particular decay channels of the untagged tau. New sources of systematic error will come from one tau decay channel polluting another one, and uncertainties on detector acceptances, but not from $q \bar{q}$ events that we have already estimated.

Table 8.4 Sequential acceptance of $\tau$ Tagging Cuts

\begin{tabular}{|c|c|c|}
\hline Cut Description & $\tau \bar{\tau} \rightarrow 3 \pi-e$ & $q \bar{q}$ \\
\hline 4 or 6 charged tracks in event & 0.70 & 0.31 \\
Sum of charges in event $=0$ & 0.98 & 0.89 \\
Hemis. has exactly $3 \pi$ and $0 \gamma$ & 0.88 & 0.022 \\
$m_{3 \pi}<m_{\tau}$ & 1.00 & 0.79 \\
$\cos \theta_{\tau-l a b}<.8$ & 0.81 & 0.66 \\
$250 \mu<l<1500 \mu$ & 0.36 & 0.04 \\
\hline Total Acceptance & 0.18 & 0.00012 \\
\hline
\end{tabular}




\section{REFERENCES}

1. M. Perl et al., Phys. Rev. Lett. 35, 1489 (1975).

2. B.C. Barish and R. Stroynowski, Phys. Rep. 157, 1 (1988);

D.G. Hitlin, Nucl. Phys. (Proc. Suppl) B3, 179 (1988); C. Kiesling, in High Energy Electron Positron Physics, eds. A. Ali and P. Söding, World Scientific, Singapore, 1988, p. 716.

3. Particle Data Group, G. P. Yost et al., Phys. Lett. 204B, 1 (1988).

4. H. Albrecht et al., Phys. Lett. 202B, 1494 (1988).

5. J. J. Gomez-Cadenas, in Proc. Tau Charm Factory Workshop, SLAC-343 (1989).

6. J. J. Gomez-Cadenas, SLAC-PUB-5019, SCIPP 88/32, submitted to Phys. Rev. D.

7. N. Cabibbo, Phys. Rev. Lett. 10, 531 (1963).

8. F. I. Berger and H. J. Lipkin, Phys. Rev. Lett. 59, 1394 (1987).

9. A. Pich, Phys. Lett. 196B, 561 (1987).

10. H. Marsiske, in Proc. Tau Charm Factory Workshop, SLAC-343 (1989).

11. F. J. Gilman and S. H. Rhie, Phys. Rev. D31, 1066 (1985).

12. F. J. Gilman, Phys. Rev. D35, 3541 (1987).

13. D. Wyler, in Proposal for an Electron Positron Collider for Heavy Flavour Particle Physics and Synchrotron Radiation, PSI PR-88-09 (1988).

14. F. Scheck, Phys. Rep. 44, 187 (1978).

15. W. Fetscher, H.-J. Gerber and K. F. Johnson, Phys. Lett. 173B, 102 (1986).

16. L. Michel, Proc. Phys. Soc. A63, 514 (1950).

17. H. Janssen et al., DESY 89-054, SLAC-PUB-4958, submitted to Phys. Lett. B.

18. Y. S. Tsai, Phys. Rev. D4, 2821 (1971). 
19. W. Fetscher, in Proposal for an Electron Positron Collider for Heavy Flavour Particle Physics and Synchrotron Radiation, PSI PR-88-09 (1988);

W. Fetscher, in Proc. Tau Charm Factory Workshop, SLAC-343 (1989).

20. W. J. Marciano and A. Sirlin, Phys. Rev. Lett. 61, 1815 (1988). 


\section{Charm Physics}

\subsection{INTRODUCTION}

There are a number of important questions in charm physics that cannot be addressed with present data samples. The asymmetric $B$ factory is an ideal machine to explore most of these questions
$\mathrm{T}$ he decays and spectroscopy of charmed particles have been studied intensively and with great success in recent years. The charmed quark is sufficiently massive that some aspects of perturbative QCD are applicable, although the corrections are larger than in bottom physics. A remarkably detailed picture of the charm decay mechanism exists, based on a large number of measured branching ratios, and lifetimes with precision of a few percent. ${ }^{1}$ Accurate measurements of the dominant semileptonic decays have been made, and much theoretical work is being done on their description. The lowest lying mesons and baryons have been observed, and higher resonances are beginning to show up.

It is relatively easy to identify a number of topics in charm physics which remain very interesting, but which are beyond the reach of present experiment:

1) $D^{0}-\overline{D^{0}}$ Mixing, and Doubly Cabibbo-Suppressed Decays;

2) CP violation;

3) Semileptonic decays;

4) $D_{s}$ and $\Lambda_{c}$ decays;

5) the pseudoscalar decay constant, $f_{D}$;

6) rare and forbidden leptonic decays;

7) meson spectroscopy; and

8) baryon spectroscopy and decays.

Of these eight topics of current interest, all but $f_{D}$ can be studied with great sensitivity at an asymmetric $B$ factory. This omission is amply compensated for by the $B$ factory's ability to study the related and perhaps more interesting quantity, $f_{B}$.

Our present experimental knowledge comes from three different types of experiments, each with its own strengths. ${ }^{1}$ Electron-positron colliders at charm threshold have been a major source of charm physics since the initial discovery of 
charmed particles in 1976. Most recently, Mark III has been the only experiment operating in this region, producing a great amount of information on the weak decays. Mark III has made some of the best measurements of hadronic and semileptonic decays $D$ decays and hadronic $D_{s}$ decays, and has produced the best limit on $\mathrm{f}_{D}$. The Beijing Electron Positron Collider should collect a charm sample about 10 times the size of Mark III's in 1991.

Fixed target experiments contributed initially only to lifetime measurements, but are now competitive in most areas of decays and spectroscopy. The Fermilab photoproduction experiment E691 has made some of the most sensitive measurements of mixing, semileptonic decays, $D_{s}$ and $\Lambda_{c}$ decays, rare leptonic decays, and excited charmed mesons. Experiments such as Fermilab E687 and E791 will be able to study charm samples an order of magnitude larger than those from the 1990 running cycle, and should therefore make major contributions to charm physics. The proposed upgrades to the Tevatron would increase hadronically-produced charm samples by another order of magnitude.

The third type of experiment to contribute to charm studies is at $e^{+} e^{-}$colliders above threshold. In the last year there has been a number of impressive results from CLEO and ARGUS operating in the upsilon region. ${ }^{2}$ They have contributed a great deal in the areas of $D_{s}$ and $\Lambda_{c}$ decays, and completely dominate meson and baryon spectroscopy. The number of events in most modes are comparable to those seen in E691. With the increased luminosity of CESR and an improved detector, the CLEO II experiment will be one of the dominant sources of charm physics in the next few years.

Rather surprisingly, no one of these three types of experiments dominates the experimental study of charm. New experiments of each kind will improve the situation over the next few years. To go well beyond these experiments will require new accelerators with much higher intensity.

An asymmetric $B$ factory of high luminosity has many advantages as a laboratory for high statistics studies of charm. With one year of running at a luminosity of $3 \times 10^{33} \mathrm{~cm}^{-2} \mathrm{~s}^{-1}$, the samples of reconstructed charm are about 200 times that
Experiments at threshold, in the $\Upsilon$ region and at fixed target machines are all roughly comparable in their contributions to our present state of knowledge in the charm sector 
The charm samples from an early run at the proposed $B$ factory are a factor of 200 larger than the largest now available. The combination of boosted charmed particles and a vertex detector adds the capability to measure lifetimes and to reduce backgrounds by using the vertex information of E691 and CLEO, and even more relative to Mark III. In addition, the combination of data is relatively rich in charm and the use of precise vertex detection should make it possible to reduce backgrounds and be sensitive to modes with very small branching ratios. The vertex detectors also add the capability of precise lifetime measurements and very sensitive searches for mixing and Doubly Cabibbo-Suppressed Decays (DCSD).

In the following we will give a few examples of the charm physics possible with the proposed machine. In many cases we are simply scaling up the rates from existing signals observed in CLEO and ARGUS, and using the fact that lifetime information is available. The general result is that the level of charm physics accessible with the $B$ factory is at least an order of magnitude beyond the leading experiments of the period 1990-92, and competitive with any machine or experiment proposed for 1994.

\section{2. $D^{0}-\overline{D^{0}}$ MIXING}

Although $D^{0}$ mixing is small in the Standard Model, it is quite sensitive to new physics, such as extended Higgs models. ${ }^{3}$ For the case of small mixing, with no $\mathrm{CP}$ violation, the rate for $K^{+} \pi^{-}$decays of a beam initially prepared as $D^{0}$ 's is

$$
\mathrm{I}\left(\mathrm{D}^{0} \rightarrow \mathrm{K}^{+} \pi^{-}\right)=\mathrm{e}^{-\Gamma \mathrm{t}}\left\{\frac{\mathrm{t}^{2}}{4}\left[\Delta \mathrm{M}^{2}+\left(\frac{\Delta \Gamma}{2}\right)^{2}\right]+\rho^{2}+\rho \mathrm{t} \frac{\Delta \Gamma}{2}\right\},
$$

where $\rho$ is the ratio of the amplitude for Doubly CabibboSuppressed Decays (DCSD) to that for allowed decays, $\Delta M=$ $M_{\text {odd }}-M_{\text {even }}$, and $\Delta \Gamma=\Gamma_{\text {odd }}-\Gamma_{\text {even }}$ (odd and even refer to the CP eigenstates). Note that the mixed events have the characteristic time dependence $t^{2} e^{-\Gamma t}$, which makes it possible to separate mixing from DCSD. Typically $\rho^{2}=\tan ^{4} \theta_{c}$ is the level of DCSD expected, although this may vary by factors of 2 or more in particular modes.

The mixing parameter $\mathrm{r}_{D}$, which is the ratio of mixed to unmixed, is equal to $\frac{1}{2}\left[\left(\frac{\Delta M}{\Gamma}\right)^{2}+\left(\frac{\Delta \Gamma}{2 \Gamma}\right)^{2}\right]$ in the limit of small 
mixing. The standard perturbative calculation, based on the diagrams shown in Figure 9.1, gives a prediction of $\mathrm{r}_{\mathrm{D}}=10^{-7}$. In contrast to the case of $B$ mixing, however, there are longdistance contributions $\mathrm{w}$ ith intermediate meson states such as $\mathrm{KK}$ and $\pi \pi$ which should dominate. ${ }^{4}$ These contributions cannot be calculated accurately, but probably give $\mathrm{r}_{D}$ in the range $10^{-4}$ or so. It is important to push the limit on $D^{0}-\overline{D^{0}}$ mixing into this range, since it is a sensitive probe of new physics. At or below this level, it would be useful to measure the mixing, although it would probably be a determination of the size of the long-distance contributions.
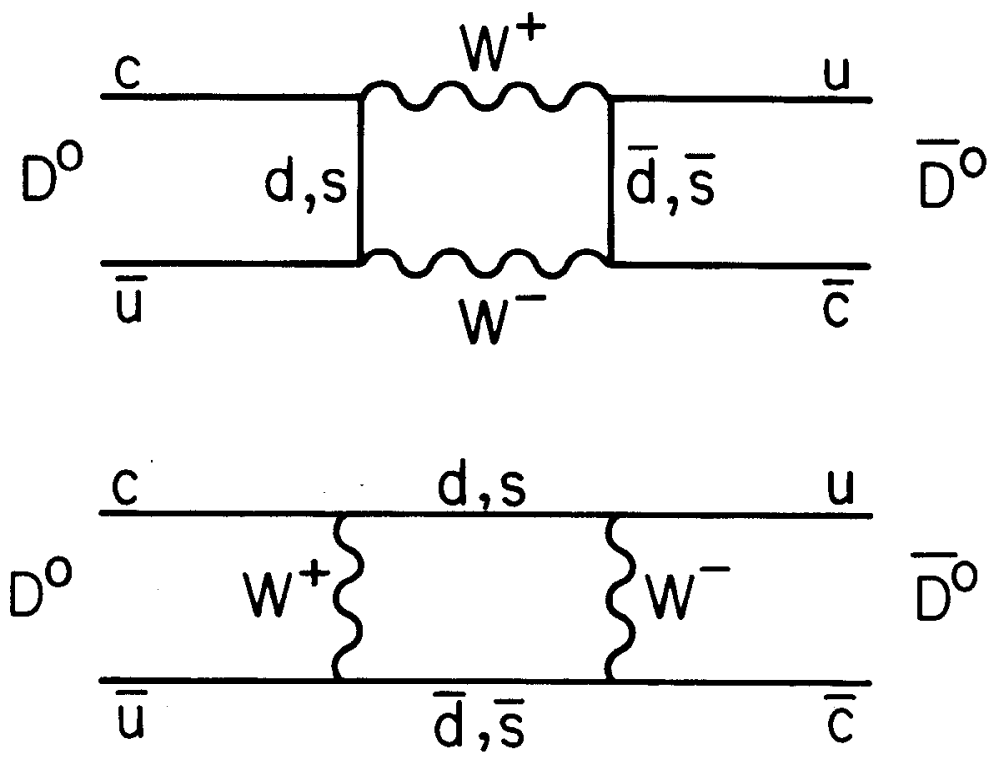

There are two techniques for tagging the charm of the $D^{0}$ meson at production. One is to use doubly tagged events, in the way Mark III has done in the $D^{0}$ system, and CLEO and ARGUS have done in the $B^{0}$ system. The other way is to use the decay process $D^{*+} \rightarrow \pi^{+} D^{0}$, which E691 used to set the best existing limits on $D^{0}$ mixing. ${ }^{5}$ Not only does it give a tag of the charm of the $D$ at production without reconstructing the other $D$ meson, but it reduces the background by a large factor. In addition, the measurement of the $D^{0}$ decay time separates mixing from DCSD.

The expected rate for observing the decay $D^{*+} \rightarrow \pi^{+} D^{0}$ is straightforward to estimate. A sample of $30 \mathrm{fb}^{-1}$ yields $3 \times 10^{7} B \bar{B}$ events and $3.5 \times 10^{7} \mathrm{c} \bar{c}$ events, corresponding to
$D^{0}-\overline{D^{0}}$ mixing above the level of $\mathrm{r}_{D}=10^{-4}$ could be an indication of new physics. It is important that measurements be pushed to this level of sensitivity

Figure 9.1 Box diagram short distance contributions to $D^{0}-\overline{D^{0}}$ mixing. 
Figure 9.2 (a) The scatter plot of $Q$ vs. $M(K \pi)$ for $\left(K^{-} \pi^{+}\right) \pi^{+}$events of Reference 5 , with the requirement $t>0.88$ ps. (b) The plot for $\left(K^{-} \pi^{+}\right) \pi^{-}$events with $t>0.88$ ps. a total of $1.8 \times 10^{7} D^{*+} \rightarrow \pi^{+} D^{0}$ decays (including charge conjugate).

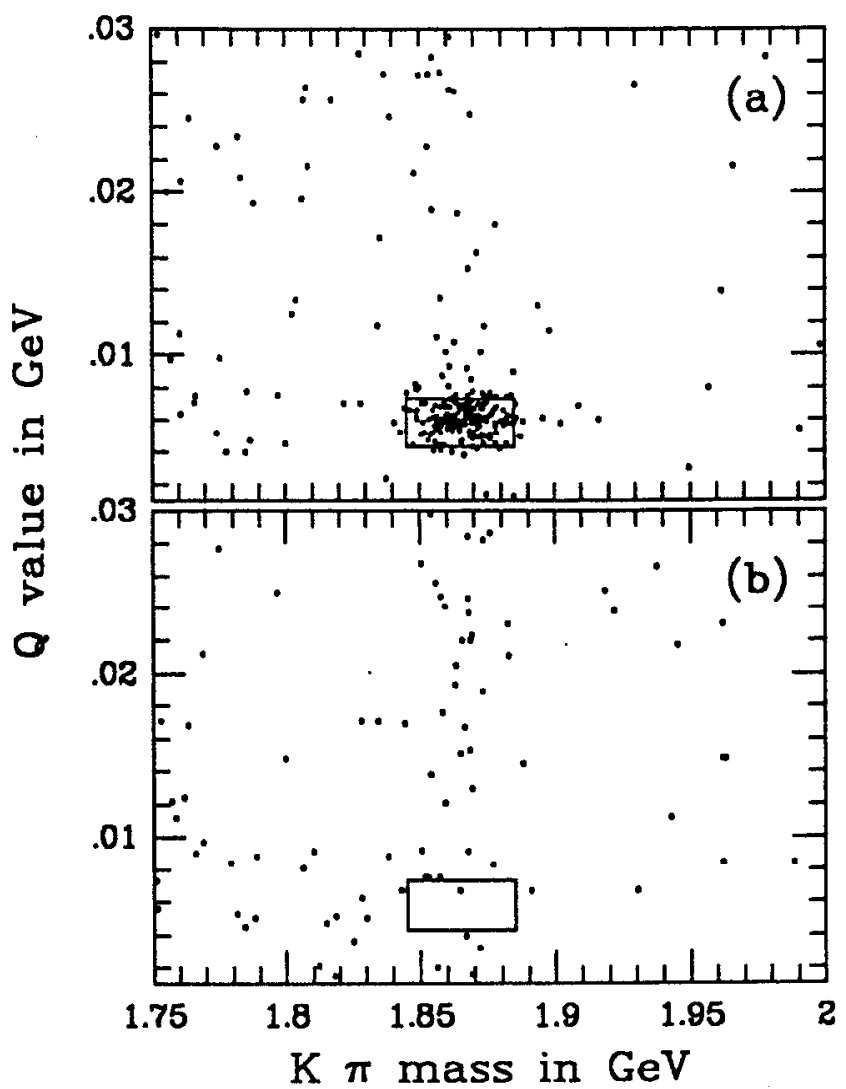

Including a total branching ratio for the $D^{0}$ into $K^{-} \pi^{+}$ or $K^{-} \pi^{+} \pi^{-} \pi^{+}$of $13 \%$, and an efficiency of $45 \%$ for $K \pi$ and $18 \%$ for $K \pi \pi \pi$, the total number of observed $D^{*+}$ events is about $6 \times 10^{5}$. These efficiencies are an average over the $c \overline{\mathrm{c}}$ events, in which the charm is produced at high momentum, and $B \bar{B}$ events, in which the efficiencies are lower. This is the denominator for the mixing parameter $\mathrm{r}_{D}$, which is the ratio of wrong sign to right sign decays. For comparison, the number of $D^{*+}$ decays observed in the E691 mixing experiment was about 3000 .

The enormous advantage in studying $D^{0}$ mixing with measurement of the decay time is that one can cut at very long decay time and still have good efficiency for the mixed $D$ 's, because of the $t^{2}$ term in the time dependence. In fact, one 
gets the best sensitivity with a cut on minimum decay time of $2.7 \tau$, although one can do better than this by fitting the full spectrum. For the present study, we will use a cut of $2.5 \tau$, or 1 picosecond. This cut also makes it easier to separate the $D^{0}$ decay products from the other tracks in the event, especially in the $D \rightarrow K \pi \pi \pi$ decay. Figure 9.2 shows the E691 scatter plot of $\mathrm{M}(K \pi)$ vs. $\mathrm{Q}[\mathrm{Q}=\mathrm{M}(\mathrm{K} \pi \pi)-\mathrm{M}(\mathrm{K} \pi)-\mathrm{M}(\pi)=5.9$ $\mathrm{MeV}$ for $D^{*+} \rightarrow \pi^{+} D^{0}$ ] for events with proper decay time greater than 2 lifetimes. The largest background is from real $D^{0}$ decays with a random pion, as shown by the band at the $D^{0}$ mass in both plots.

The efficiencies and backgrounds for the process decay sequence $D^{*+} \rightarrow \pi^{+} D^{0} \rightarrow K^{-} \pi^{+}$have been estimated with the parameterized $\mathrm{M}$ onte Carlo simulation and by scaling from existing experiments. Figure 9.3(c) shows the signal from ARGUS, for events in the region $x>0.5$. This, and similar plots from CLEO, can be used to estimate background levels. Since the $D^{0}$ sample is mostly due to the signal, the background is from random pions combined with real $D^{0}$ 's. The figure of merit for background is the ratio of random $\pi^{+} D^{0}$ combinations within the resolution to the real $D^{*+}$ combinations. This ratio is $\frac{\mathrm{B}}{\mathrm{S}}=\frac{1}{\mathrm{~N}_{\mathrm{s}}} \frac{\mathrm{dN}_{\mathrm{B}}}{\mathrm{dQ}} \Delta \mathrm{Q}$, where $\Delta \mathrm{Q}$ is the accepted range, $N_{s}$ is the number of real $D^{*+}$ decays, and $\frac{d N_{B}}{d Q}$ is the number of accidental combinations per unit of $\mathrm{Q}$. The plot from ARGUS suggests $\frac{1}{N_{s}} \frac{d N_{B}}{d Q} \simeq 0.008$ background event per $\mathrm{MeV}$ per real $D^{*}$. In a study of mixing they reduce their background further with tighter cuts, to a level of $0.002 .{ }^{6}$ The mass plot for identified $\mathrm{K}^{-} \pi^{+}$combinations from the Monte Carlo is shown in Figure 9.4. There are events from $c \bar{c}$ production with $\mathrm{x}>0.3$, and the $D^{0}$ does dominate the signal region. The background from light quark events is comparable to that shown, but is reduced further by cuts on the vertex topology of the event to a smaller level. When these $\mathrm{K}^{-} \pi^{+}$ combinations are attached to all other $\pi^{+}$tracks, the $\mathrm{Q}$-value distribution shown in Figure 9.5 is obtained. The background level is $\frac{1}{N_{s}} \frac{d N_{B}}{d Q}=0.004$, consistent with CLEO and ARGUS. 
Figure 9.3 Plots of $D^{0} \pi^{+}$ mass from ARGUS for
(a) $D^{0} \rightarrow K^{+} \pi^{-}$,
(b) $D^{0} \rightarrow K^{+} K^{-}$, and
(c) $D^{0} \rightarrow K^{-} \pi^{+}$.
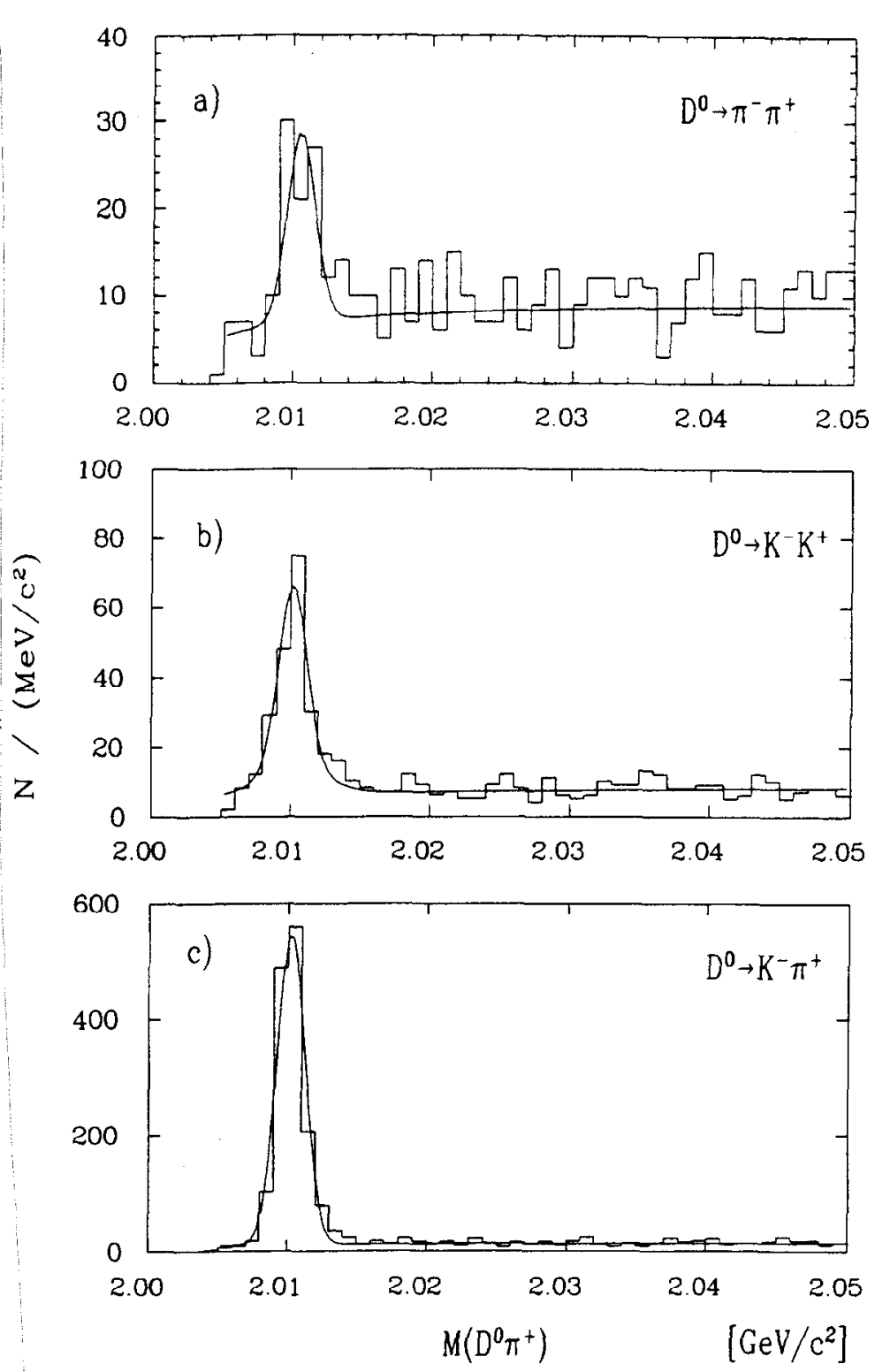

The resolution, however, is somewhat better; $\sigma(\mathrm{Q})=0.3$ $\mathrm{MeV}$ rather than 0.8 . This is due to a precise measurement of the angle of the very soft pion from the $D^{*}$ decay in the silicon detectors. By using the $D^{0}$ to measure the source of the pion with good accuracy, the angle of the pion can be measured before the large multiple scattering in the beam pipe and silicon, which would further improve this resolution. The acceptance for the soft pion is limited at low pr by the ability to reconstruct the track after it loses a significant fraction of 
its energy in the material. This is not too bad for the high momentum $D^{*}$ s, but becomes important at low $\mathrm{x}$.

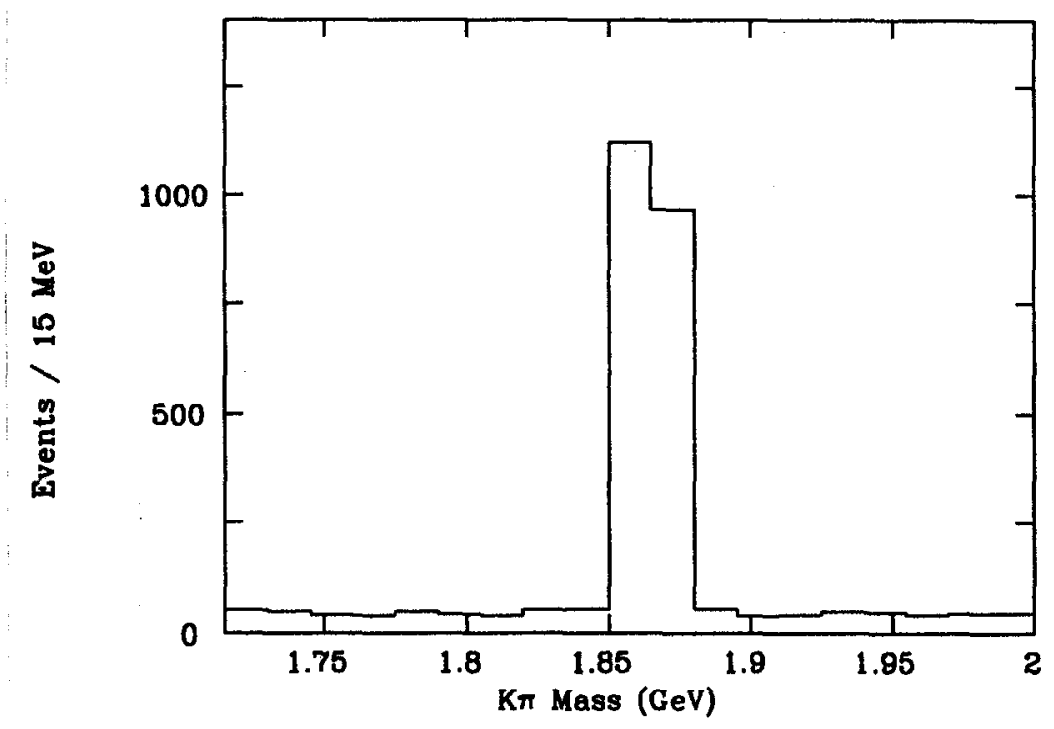

Based on these numbers one can estimate the expected sensitivity. Of the $6 \times 10^{5} D^{*+}$ decays seen, the $D^{0}$ survives a cut at 2.5 lifetimes in only $5 \times 10^{4}$ cases. The resolution in decay time is about $0.3 \tau$, which is better than needed for this analysis. Thus the expected wrong sign background within a region of $0.8 \mathrm{MeV}$ in $\mathrm{Q}$ should be about 160 events. The fluctuations in this smooth spectrum set the expected mixing limit at $90 \%$ confidence level to be about 20 events. Since this cut keeps $54 \%$ of the mixed decays, the mixing limit is

$$
\mathrm{r}_{D}<37 /\left(6 \times 10^{5}\right)=6 \times 10^{-5}
$$

This is a full factor of 80 beyond the present E691 limit and is well within the range expected from conventional sources. The only difficult parts of this estimate are the detection efficiency, and the number of pions within $1.5 \times$ the resolution in $Q$ value. The limit only depends on the square root of these two numbers, so the estimate should be reasonably robust.
Figure 9.4 The Monte Carlo $K^{-} \pi^{+}$mass spectrum for $50,000 c \bar{c}$ events, with $x>0.4$.

The resulting limit, based on Monte Carlo results which agree with ARGUS experience, would be $\mathrm{r}_{D}<6 \times 10^{-5}$, which is within the range expected from the Standard Model 
Figure 9.5 The Q-value spectra obtained by combining $D^{0}$ candidates from Figure 9.4 with pions, to see the decay $D^{*+} \rightarrow \pi^{+} D^{0}:$ (a) $\pi^{+} D^{0}$ and (b) $\pi^{+} \bar{D}^{0}$.
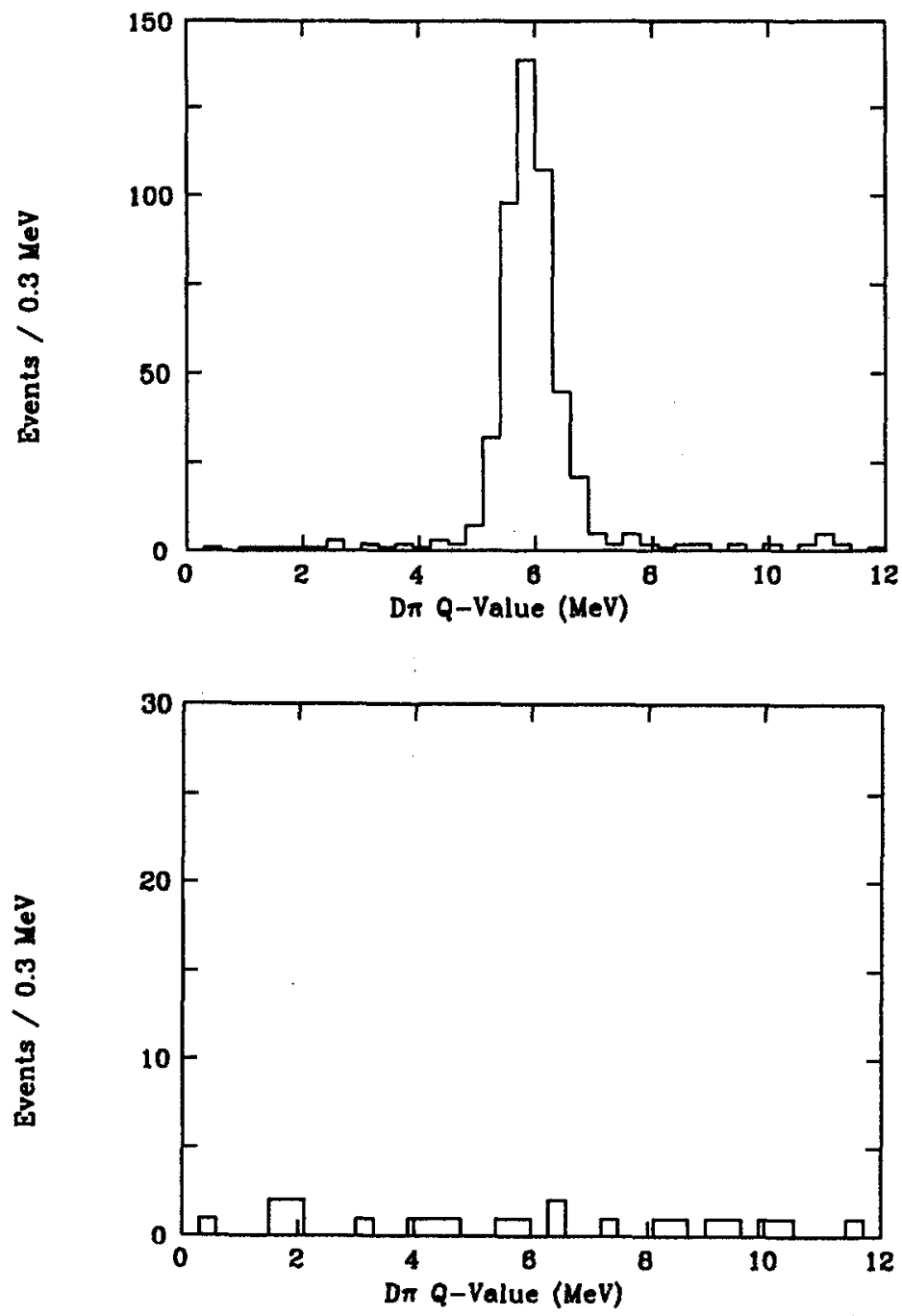

The other background in observing mixing is itself an interesting physics process, the previously unseen Doubly Cabibbo-Suppressed Decays. Assuming $\rho=2.5 \times 10^{-3}$, there would be about 1,500 such decays seen, over a wrong sign background of about 4,000 events, which would be a spectacular signal. Despite this relatively large source of wrong sign decays, there would not be a large reduction in sensitivity to mixing. Only 120 of these DCSD events have decay times beyond $2.5 \tau$, which is less than the 160 events from random pion contamination. The number of DCSD events is well measured for each mode at short decay times, so the extrapolation to $t>2.5 \tau$ can be done with small additional error. If large mixing exists due to new physics, it would be 
due to large $\Delta \mathrm{M}$, with $\Delta \Gamma<<2 \Delta \mathrm{M}$, because the intermediate states are not energetically allowed decay modes. ${ }^{3}$ In that case the interference term in Equation (1) is negligible, and the mixing parameter $\mathrm{r}_{D}$ is extracted as shown.

At the level of $10^{-4}$ expected in the Standard Model from intermediate meson states, one expects the interference term to contribute, and it will be included in the fit of the decay time spectrum. The limit will be given by a contour in $\Delta \Gamma$, $\Delta \mathrm{M}$ space, and will typically be more sensitive if $\Delta \Gamma \neq 0$. If mixing is seen, such information about the interference will be useful in sorting out the source. Experimental information on the branching ratios of $D^{0}$ into $\mathrm{CP}$ eigenstates which will be available by the time of the $B$ factory will allow reasonably good calculations of $\Delta \Gamma{ }^{3}$ Mixing can also be studied in modes free of any DCSD decays, $D^{0} \rightarrow \mathrm{K}^{-} \ell^{+} \nu_{\ell},(\ell=e, \mu)$, each of which has a branching ratio equal to that of $D^{0} \rightarrow K^{-} \pi^{+}$. The efficiencies should be close to that of $D^{0} \rightarrow K^{-} \pi^{+}$, and the background is probably low enough at long decay times that real $D^{0}$ 's combined with random pions dominate the wrong-sign $D^{*+}$ background, as they do for $D^{0} \rightarrow K^{-} \pi^{+}$.

The separation of DCSD from the "background" of Cabibbofavored and SCSD decays is more difficult than for mixing, because the time dependence is not useful. At rest, a $D^{0}$ decaying into $K^{-} \pi^{+}$has the same mass when identified as $K^{+} \pi^{-}$. On the other hand, for moving $D$ 's at a $B$ factory, there is a mass shift for this misidentification of $\Delta \mathrm{M}=$ $65 \mathrm{MeV} \cdot\left[4 \beta \cos \theta /\left(1-\beta^{2} \cos ^{2} \theta\right)\right]$, which for a typical $\beta \simeq 0.8$ gives a broad band about $300 \mathrm{MeV}$ wide. It is possible to cut out all events consistent in mass with $D^{0} \rightarrow K^{-} \pi^{+}$while only slightly reducing the efficiency for the properly identified DCSD decays, or distorting the smooth background beneath them. This cut, together with particle identification cuts, provides the necessary suppression of the Cabibbo-favored mode, and the nearby satellite peak from the $K^{-} \pi^{+} \pi^{0}$ final state, to the level that they simply make a small contribution to the smooth background underneath the $\mathrm{K}^{+} \pi^{-}$peak. 


\subsection{CP VIOLATION}

There is also some interest in looking for anomalously large $\mathrm{CP}$ violation in $D^{0}$ decays. Golden and Grinstein, ${ }^{7}$ and Bigi, ${ }^{3}$ have separately pointed out that for a variety of new physics one can obtain $\mathrm{CP}$ asymmetries of as large as $10 \%$ for decays of $D^{0} \rightarrow K^{+} K^{-}$or $\pi^{+} \pi^{-}$. The experimental observation that $\mathrm{B}\left(D^{0} \rightarrow K^{+} K^{-}\right) / \mathrm{B}\left(D^{0} \rightarrow \pi^{+} \pi^{-}\right) \simeq 3$ leads to the hope that penguin diagrams are interfering with the usual spectator diagrams. Such an interference would permit the $\mathrm{CP}$ violation to manifest itself, even if mixing is small.

We can search for an anomalously large effect in $D^{o} \rightarrow K^{+} K^{-}$ or $\pi^{+} \pi^{-}$at the $1 \%$ level
Again the decay $D^{*+} \rightarrow \pi^{+} D^{0}$ provides a self-tagging method of identifying $D^{0}$ 's. ARGUS has obtained clean signals of $57 \pm 9 D^{0} \rightarrow \pi^{+} \pi^{-}$and $131 \pm 20 D^{0} \rightarrow K^{+} K^{-}$in their $200 \mathrm{pb}^{-1}$ sample of data from the $\Upsilon$ region (see Figure 9.3). With the initial sample of $30 \mathrm{fb}^{-1}$, it would be possible to obtain about $10,000 D^{0} \rightarrow \pi^{+} \pi^{-}$decays and 20,000 $D^{0} \rightarrow K^{+} K^{-}$decays, tagged as coming from a $D^{*+}$ or $D^{*-}$. With this sample, it is possible to measure the $\mathrm{CP}$ violation asymmetry $\mathrm{B}\left(D^{0} \rightarrow K^{+} K^{-}\right) / \mathrm{B}\left(\bar{D}^{0} \rightarrow K^{+} K^{-}\right)$to $1 \%$.

While the capabilities of the charmed meson data sample obtained by a $B$ factory for observing CP violation in the charm sector are quite comparable to those for a data sample taken near threshold, it must be emphasized that the physics interest of this search does not compare with the search for $\mathrm{CP}$ violation in $B$ meson decay. First, the Standard Model prediction for the size of CP-violating effects in the charm sector is almost certainly unobservably small. While finding a positive effect would thus be of uncommon interest, it is most unlikely. Second, the great virtue of the program to search for CP violation in $B$ decay to CP eigenstates, namely that the results are not clouded by hadronic corrections, and may therefore be used with confidence to test the consistency of Kobayashi-Maskawa parameters, does not obtain in the charm sector, where hadronic corrections are dominant. 


\subsection{Semileptonic Decays of Charm}

As in the case of the bottom quark, semileptonic decays of the charmed quark are particularly interesting, because they are relatively easy to interpret theoretically. Measurements of exclusive semileptonic decays provide the best determination of $V_{c s}$ and $V_{c d}$, the Kobayashi-Maskawa matrix elements. In addition, the form factors measured in these decays give important information on the wave function of mesons including one heavy quark. There has been a great amount of theoretical work in the last year, using both analytical and lattice gauge techniques, on models of the form factors in semileptonic decays of $B$ and $D$ mesons.

The decay diagrams for semileptonic charm decays are shown in Figure 9.6 (a) for Cabibbo-favored, (b) for Cabibbosuppressed decays. The form factors describe the amplitude for the sq state to become a $K, K^{*}$, or $K^{* *}$ in the final state. One of the crucial issues is to determine the dependence of the form factors on the momentum transfer $\mathrm{q}^{2}=\mathrm{M}_{\mathrm{e} \nu}^{2}$. The present models usually assume that the form factors are described by a single pole of the form $f\left(q^{2}\right)=\frac{f_{0}\left(q^{2}\right)}{1-q^{2} / M_{0}^{2}}$ where $M_{0}$ is the mass of the lowest lying meson with the appropriate spin-parity. Although this assumption is not very important for decays such as $B \rightarrow D e \nu$, it becomes the dominant uncertainty in the case of $B \rightarrow \pi e \nu$, where the $\mathrm{q}^{2}$ dependence changes the rate by a factor of 20 from $q^{2}=0$ to $q_{\max }^{2}$, if the single $B^{*}$ pole dominates. It is, therefore, impossible to measure $\mathrm{V}_{\mathrm{ub}}$ without understanding the form factor dependence on $\mathrm{q}^{2} .{ }^{8}$ The charm decays $D \rightarrow \pi e \nu$ and $D \rightarrow K e \nu$ are considered to be similar to $B \rightarrow \pi e \nu$, because of the light mass of the final state quark.

The rates for such standard decays as $D \rightarrow K^{*} e \nu$ are quit e striking. The best measurement that exists for this mode is from $\mathrm{E} 691$, in the decay mode $D^{+} \rightarrow \bar{K}^{* 0} e^{+} \nu$, with a sample of $200 \mathrm{ev}$ ents. At the asymmetric $B$ factory, with an integrated luminosity of $30 \mathrm{fb}^{-1}$, there are about $9 \times 10^{5}$ decays $D^{+} \rightarrow \bar{K}^{* 0} e^{+} \nu$, bar $K^{* 0} \rightarrow K^{-} \pi^{+}$. The efficiency for this mode should be good even with tight vertex cuts, because of the long lifetime of the $D^{+}$. Scaling from other charm modes leads to a total of about $10^{5}$ decays, with complete 
Figure $9.6 \quad$ Semileptonic decay diagrams for the non-strange $D$ 's:

(a) Cabibbo-favored spectator, (b) Cabibbo-suppressed spectator.

Definitive measurements of Cabibbo-allowed and -suppressed semileptonic decays are possible coverage of the kinematic range, which would make it possible to determine the three form factors with great accuracy. The backgrounds for this mode are only about $5 \%$ in the case of E691, and should be comparably small at the $B$ factory.

D

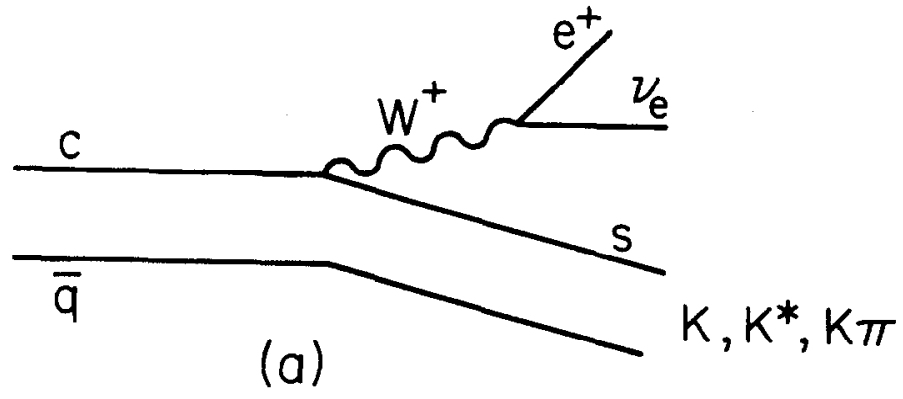

D

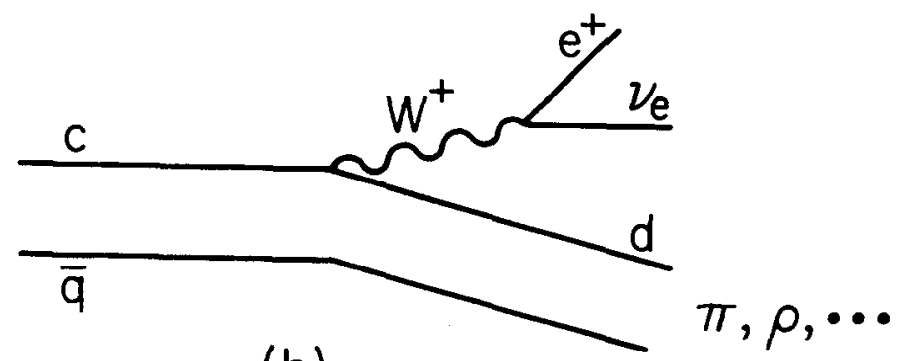

(b)

A more challenging experiment is the decay $D^{0} \rightarrow \pi^{-} e^{+} \nu_{e}$, which is used to measure $V_{\text {cd }}$. It also presents a chance to study the $q^{2}$ dependence very near the dominant pole, since $q_{\max }^{2} \simeq 3.0 \mathrm{GeV}^{2}$ and the lowest vector meson pole has $\mathrm{M}^{2}\left(D^{*}\right)=4.0 \mathrm{GeV}^{2}$. The technique will be to use $D^{*+} \rightarrow$ $\pi^{+} D^{0}, D^{0} \rightarrow \pi^{-} e^{+} \nu_{e}$ decays, of which there are $7 \times 10^{4}$ in the standard run. The only background of importance is the decay $D^{0} \rightarrow K^{-} e^{+} \nu_{e}$, wh ich has a rate ten times larger. Thus the requirement on $K \rightarrow \pi$ misidentification is not very demanding; especially since one can restrict the $\pi$ to have momentum less than $1.2 \mathrm{GeV} / \mathrm{c}$ without too much loss of acceptance. Because of the wide momentum range for the $D^{0}$ in the laboratory frame at a $B$ factory, the entire $q^{2}$ range is covered even with such cuts on the laboratory momentum. The lessons learned about the form factors in this case should improve our understanding of the $B^{0} \rightarrow \pi e \nu$ case needed to measure $\mathrm{V}_{\mathrm{ub}}$. 


\subsection{MESON SPECTROSCOPY}

Two new areas of interest in charm physics are the spectroscopy of the $\mathrm{L}=1 \mathrm{D}^{* *}$ meson resonance and charmed baryons. The interest is due to the fact that experimental data is becoming available, in particular from CLEO and ARGUS. These are areas that cannot be explored at $e^{+} e^{-}$ machines operating near threshold, and are difficult in fixed target experiments.

The expected $D^{* *}$ spectroscopy is composed of 12 states. There are 4 states each from $c \bar{u}, c \bar{d}$ and $c \bar{s}$ : one $\mathrm{J}^{\mathrm{P}}=0^{+}$, two $\mathrm{J}^{\mathrm{P}}=1^{+}$, and one $\mathrm{J}^{\mathrm{P}}=2^{+}$states. Parity and angular momentum conservation require that the $0^{+}$states decay only to $D \pi(D K)$ and $1^{+}$states only to $D^{*} \pi\left(D^{*} K\right)$, while $2^{+}$ states can decay into either mode. For the strange case $D_{s}^{+} \pi$ decays are forbidden by conservation of isospin.

As pointed out by Rosner ${ }^{9}$ and others ${ }^{10,11}$ the cō system is of great interest; with the single light quark, it is analogous to the hydrogen atom. The system, especially in the P states, is sensitive to the long range confinement (scalar) part of the effective potential. Since the wave function at $r=0$ is negligible, the splitting between states is due to spin-orbit and tensor forces, rather than spin-spin forces. The total quark spin is not expected to be a good quantum number; the two $1^{+}$states are expected to mix with the physical states identified as $1_{1 / 2}$ and $1_{3 / 2}$ where the subscript is the total angular momentum of the light quark. It is important to measure the mass, width and spin of all of the states, to extract the level ordering and splittings. The two $1^{+}$states can be identified by the ratio of decays to $D \rho$ and $D^{*} \pi$ (only the lower tail of the $\rho$ is energetically possible).

The vertex topology of $D^{* *}$ production at an asymmetric $B$ factory is illustrated in Figure 9.7. In Figure 7a is shown an event in $c \bar{c}$ continuum production. Here a $D^{*+}$ is formed from a $\pi^{+}$and $D^{0} \rightarrow K^{-} \pi^{+} \pi^{+} \pi^{-}$, and a $D^{* * 0}$ is formed by combining this $D^{*+}$ with negative pions in the event. CLEO has shown that a clean $D^{*+}$ sample can be observed without using precision vertex information. The background to the
The spectroscopy of excited $D$ mesons yields information on th spin structure of the $q \bar{q}$ potential at large distances 
Figure 9.7 Event topologies for $D^{* *}$ 's produced via (a) $c \bar{c}$ events and in (b) $B$ hadronic and (c) semileptonic decays. (a)

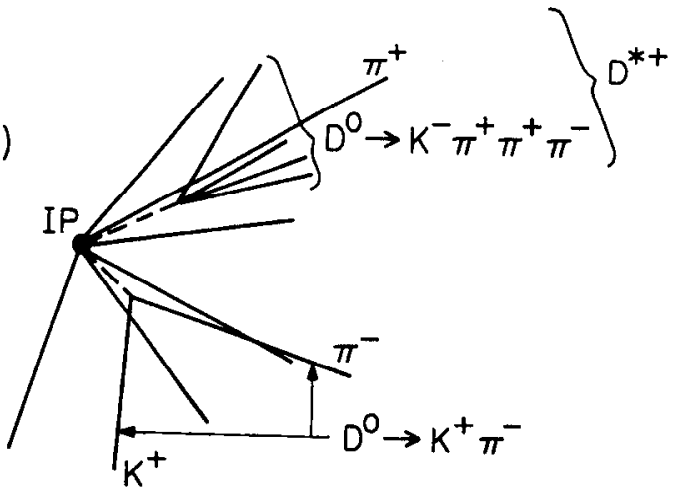

(b)

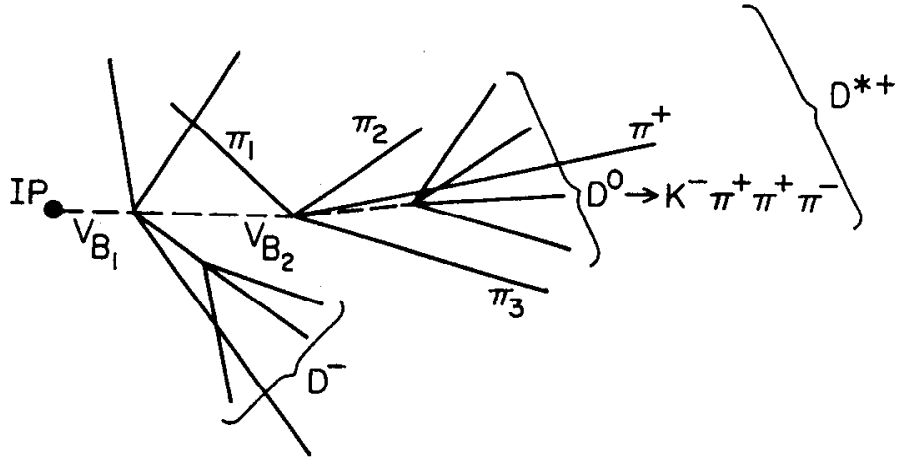

(c)

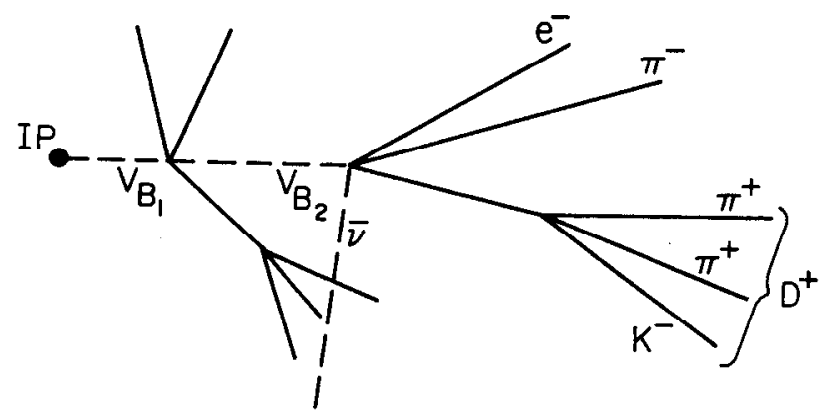

$D^{* *}$ spectrum is due to the combinations with the negative pions which emerge from the IP. As opposed to the case of CLEO and ARGUS, this could in principle be improved by not using negative pions from the other $D$ decay, as defined by the vertex detector. For the $D^{*+}$ case the vertex detector could be used to reduce the kinematic cuts on the $D^{0}$ signal and to include modes such as $D^{0} \rightarrow K^{-} \pi^{+} \pi^{0}$. For the $D^{+} \rightarrow$ $K^{-} \pi^{+} \pi^{+}$casc the vertex detector will allow a big reduction in the large background under the $D^{+}$as observed by CLEO and ARGUS.

Figure 9.7(b) shows the vertex topology of $D^{* *}$ 's from $B$ 
decays. If The two $B$ vertices are well-separated and if the $\mathrm{p}_{\mathrm{T}}$ of the $D^{* *}$ decay pion is high enough for adequate resolution in impact parameter, only the pions from the proper $B$ vertex are used to form $D^{* *}$ candidates. This will reduce the combinatorial background by a factor of 2-3.

The cleanest sample of $D^{* *}$ 's comes from semileptonic decays of B's as shown in Figure 9.7(c). For well separated $B$ vertices the only negative pion to be combined with the $D^{+}$ is the one from the vertex containing the lepton and the projection of the $D^{+}$. As for the case of $K^{*}$ 's in $D^{+} \rightarrow \bar{K}^{* 0} e^{+} \nu$, we expect the $D^{* *}$ spectrum in semileptonic $B$ decays to be extremely clean. These events should be very useful in spin parity analyses since the helicity of the $D^{* *}$ can be controlled by the lepton kinematics.

Recently ARGUS, E691, and CLEO have observed $D^{*} \pi$ states at a mass of about $2420 \mathrm{MeV} / \mathrm{c}^{2}$, with the best observation in the neutral state $D^{* * 0}(2420) \rightarrow D^{*+} \pi^{-}$. The data of Figure 9.8 are from CLEO ${ }^{12}$ A second state at a mass of 2459 has been observed best in the neutral state $D^{* * 0}(2459) \rightarrow D^{+} \pi^{-}$. Here the signal to background is significantly worse due to the large background under the $D^{+}$ peak. The CLEO peak in $D^{*+} K_{s}$ is shown in Figure 9.9. The peak in $D^{*+} \pi^{-}$is thought to be predominantly a $1^{+}$state, but with some contribution from the $2^{+}$. There are indications that the decay distribution in the $D^{+} \pi$ spectra are not isotropic, indicating that the $D^{* * 0}(2459)$ is $2^{+}$. From the observed signals it is clear that approximately $10 \%$ of the $D^{*+}$ 's or $D^{+}$'s observed in charm events originate as decay products of single $D^{* *}$ states. The most important goals are to observe the $0^{+}$and the other $1^{+}$state, which have not yet been seen, and to determine their spin and parity. 
Figure 9.8 The $D^{* * 0}(2420)$ signal of CLEO. The angular cuts indicate that the peak is probably a superposition of the $D^{* * 0}(2459) 2^{+}$state and a $1^{+}$ state of lower mass.

The CLEO and ARGUS results show the best signals for $D^{* *}$ and $D_{s}^{* *}$ mesons

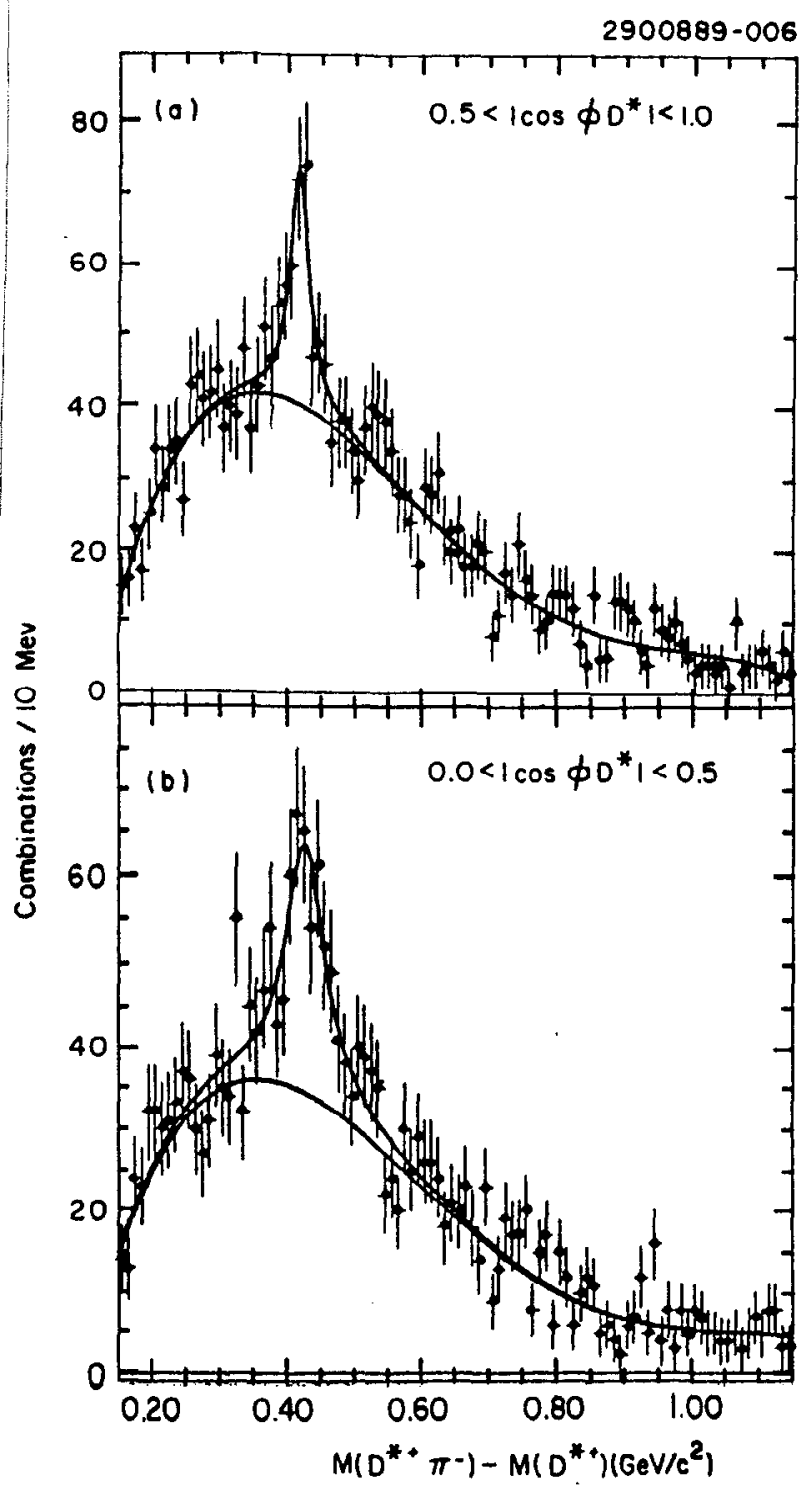

In Table 9.1 we make conservative projections of the number of $D^{* *}$ 's observable from the cec continuum. with an integrated luminosity of $30 \mathrm{fb}^{-1}$. These projections are made by scaling from CLEO results obtained with an integrated luminosity of $430 \mathrm{pb}^{-1}$. We note that all CLEO results are obtained with cuts on $\mathrm{x}$ for the $\mathrm{D} / D^{*}$ and $D^{* *}$ of 0.6 , so that the entire signal comes from the $c \bar{c}$ continuum with no contribution from $B$ decays. The $c \bar{c}$ projections for the cases with $D^{*+}$ 's assume no use of high precision vertexing, and are therefore very conservative. Vertexing will allow other 
$D^{0}$ modes (other than $K^{-} \pi^{+}$and $K^{-} \pi^{+} \pi^{+} \pi^{-}$) to be used and reduced kinematic cuts, leading to a larger sample.

The combinatorial background will be reduced somewhat by eliminating combinations with pions from the other charmed particle. For the case of $D^{+} \pi^{-}$vertexing is important and also efficient since the $D^{+}$has a long lifetime. We assume that the $D^{+}$signal is cleaned up with a signal-to-background ratio of 2 with an efficiency reduction of 0.5. Again the assumptions are conservative in that we assume only $D^{+} \rightarrow K^{-} \pi^{+} \pi^{+}$is used and that the combinations are not reduced by eliminating pions from the other charmed particles. Even with conservative assumptions it is clear that there will be an enormous signal of $D^{* *}$ 's in c $\bar{c}$ events. For the case of $D^{* *}$ 's in $B$ decays the spectra will be significantly cleaner, and the number of events smaller, depending upon the vertex resolution and cuts used.

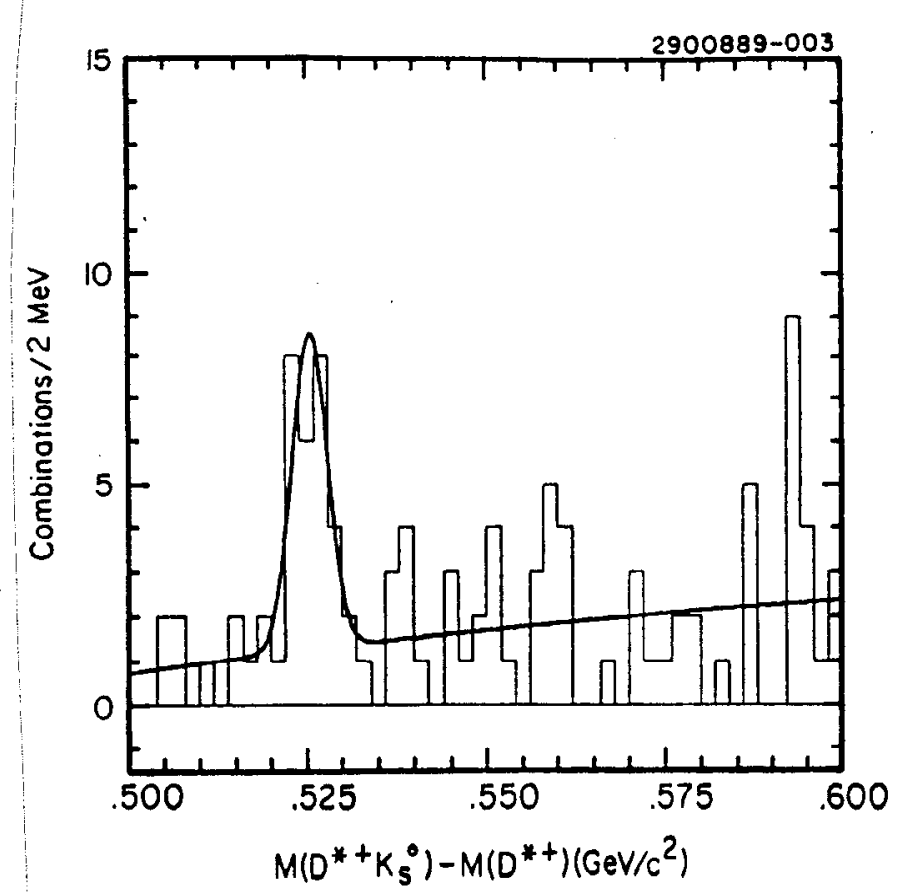

We try to estimate the difficulty of seeing the so-far unobserved states, and take the neutral $0^{+}$as an example. We assume that the reason it has not yet been observed is that it is broad and is less copiously produced. Predictions for the width vary over a wide range; we take $150 \mathrm{MeV}$ as an example. Because of spin multiplicity, we estimate that it is produced at $1 / 3$ the rate of the $D^{* *}(2420)$ which is thought to
Figure 9.9 The CLEO $D_{s}^{* *}(2535)$ peak seen in $D^{*+} K^{*}$. 
The entire spectroscopy of $l=1 \mathrm{D}$ mesons can be studied at the $B$ factory be $1^{+}$. The signal-to-background ratio in the $D^{+} \pi^{-}$spectrum would then be reduced by $\frac{1 / 3}{150 / 20}=0.04$ as compared with the estimates for the $D^{* *}(2459)$. This would give a $\mathrm{S} / \mathrm{B} \sim 0.02$ in the $c \bar{c}$ events and $S / \sqrt{B}=10$. For the unseen $1^{+}$state we assume a width of $150 \mathrm{MeV}$ and a production rate the same as the $D^{* * 0}(2420)$. This would correspond to a $\mathrm{S} / \sqrt{\mathrm{B}}$ for CLEO of about 4 . This is $1 / 2$ of the $S / \sqrt{B}$ for the CLEO signal for the $D^{* *}(2459)$ and would explain why this state has not yet been seen.

Table 9.1

\begin{tabular}{|c|c|c|c|c|}
\hline & & $\begin{array}{c}\text { CLEO }^{*} \\
430 \mathrm{pb}^{-1}\end{array}$ & $\begin{array}{r}B \mathrm{Fac} \\
30 \mathrm{fb}\end{array}$ & \\
\hline$J^{P}$ & Decay Mode & S S/B & S & $\mathrm{S} / \mathrm{B}$ \\
\hline $2^{+}$ & $D^{* * 0}(2459) \rightarrow D^{+} \pi^{-}$ & $440 \quad .15$ & 15,000 & .6 \\
\hline $2^{+}$ & $D^{* *+}(2471) \rightarrow D^{0} \pi^{+}$ & $\S$ & 7,000 & 3 \\
\hline $1^{+}$ & $D^{* * 0}(2420) \rightarrow D^{*+} \pi^{-}$ & $300 \quad .4$ & 21,000 & .4 \\
\hline $1^{+}$ & $D^{* *+}(2420) \rightarrow D^{* 0} \pi^{+\dagger}$ & & & \\
\hline $1^{+}$ & neutral $\rightarrow \mathrm{D}^{*+} \pi^{-}$ & & 21,000 & .06 \\
\hline $0^{+}$ & neutral $\rightarrow \mathrm{D}^{+} \pi^{-}$ & & 5,000 & .02 \\
\hline $2^{+}$ & $D^{0} K^{+}, D^{+} K_{s}$ & & 22,000 & 5 \\
\hline $1^{+}$ & $D_{s}^{* *+}(2535) \rightarrow D^{*+} K_{s}$ & 30 & 2,100 & 5 \\
\hline $1^{+}$ & $D^{*+} K_{s}$ & & 22,000 & .5 \\
\hline $0^{+}$ & $D^{0} K^{+}$ & & 670 & .17 \\
\hline
\end{tabular}

* Blanks indicate that the state has not been seen by CLEO $\S$ Seen by ARGUS

$\dagger$ Seen in $D^{0} \pi^{+}$by reflection $D^{* 0+} \rightarrow \pi^{+} D^{* 0}, D^{0} \rightarrow D^{0}\left(\pi^{0}, \gamma\right)$

With the $B$ factory the $1^{+}$and $0^{+}$states should both be

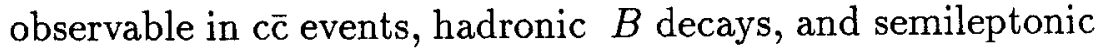
$B$ decays, if the width is less than $150 \mathrm{MeV}$. If the $0^{+}$is much wider than $150 \mathrm{MeV}$ it will be more difficult but still possible to find, and it would be invisible for all other experiments. 


\subsection{BARYON SPECTROSCOPY AND DECAYS}

Charmed baryons, like $D^{* *}$ 's, are becoming an important area of charm physics as good data becomes available. In addition to the spectroscopy of the baryons, the decays of baryons add a new dimension to the study of weak decays. The helicity suppression of $\mathrm{W}^{+}$exchanges and annihilation is not present for the spin $1 / 2$ baryons and so these mechanisms are expected to contribute with strength comparable to the spectator decay. The fact that the $\Lambda_{c}^{+}$lifetimes is about half that of the shortest lived $D$ 's is a strong indication that this picture is right, although detailed study of decay modes is just starting. CLEO and ARGUS have demonstrated that 10 $\mathrm{GeV} / \mathrm{c} e^{+} e^{-}$machines are ideal for charmed baryon physics; many decays of the $\Lambda_{c}^{+}$have now been observed. The expected singly charmed $1 / 2^{+}$baryons are shown in Figure 9.10. Nine states are expected, of which four decay weakly with lifetimes that need to be measured. The CLEO signal ${ }^{13}$ for $\Xi_{c}^{0} \rightarrow \Xi^{-} \pi^{+}$shown in Figure 9.11 represents the clearest indication that this environment is without equal for the study of charmed baryons.

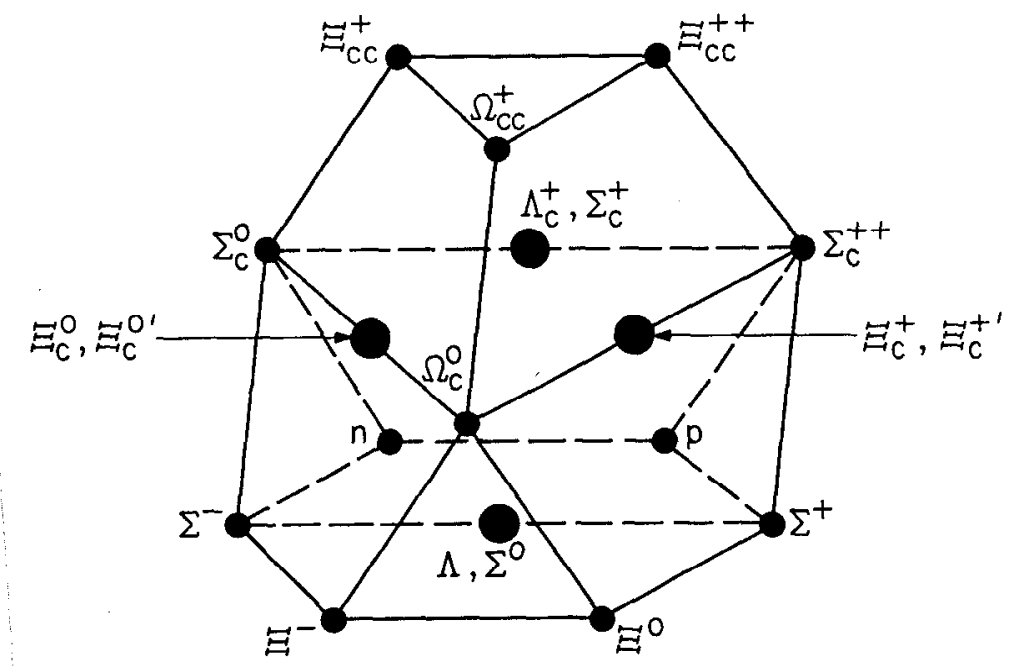

In Table 9.2 we show the characteristics of the CLEO signals and the conservative estimated numbers for a $B$ factory with $30 \mathrm{fb}^{-1}$ integrated luminosity. Precision vertexing is used only to clean up the $\Lambda_{c}^{+} \rightarrow p K \pi$ signal. The $\Lambda_{c}^{+}, \Xi_{c}^{0}, \Xi_{c}^{+}$ and $\Omega_{c}^{0}$ decays are weak; the other states decay hadronically
Studies of charmed baryons are already dominated by CLEO and ARGUS. More states will be seen at the asymmetric $B$ factory. Lifetimes for all those states which decay weakly can be measured
Figure 9.10 The $1 / 2^{+}$baryon 20 plot. There should be 9 states which have a single $c$ valence quark. Four of these states decay weakly. 
or electromagnetically depending upon the $\mathrm{Q}$ values available. The $\Xi_{c}^{\prime 0}$ and $\Xi_{c}^{+\prime}$ may not be massive enough for strong decays.

Figure 9.11 The CLEO signal for $\Xi_{c}^{0} \rightarrow \Xi^{-} \pi^{+}$.

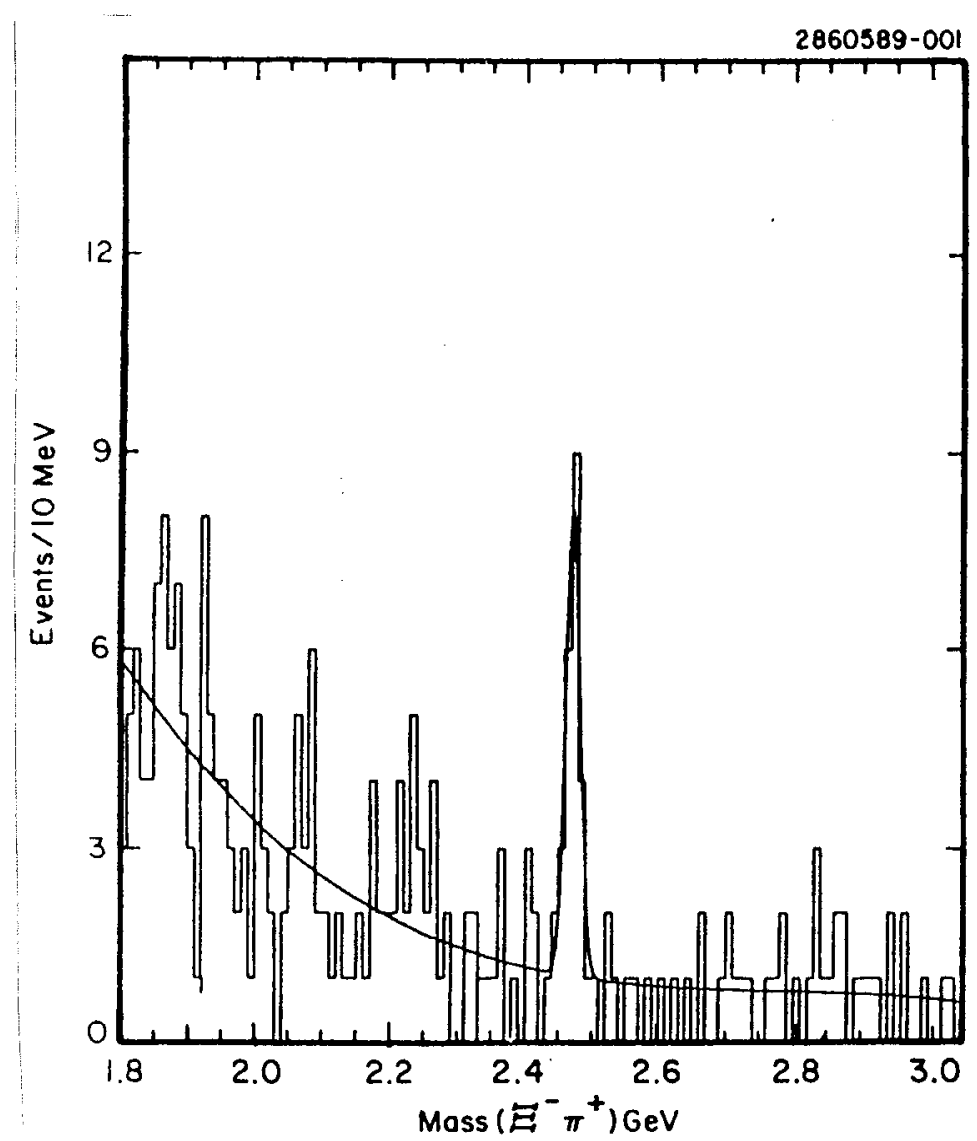

NA32 has demonstrated, with their $\Xi_{c}^{+} \rightarrow \Xi^{-} \pi^{+} \pi^{+}$signal, the advantage of observing the charged hyperons in the silicon detector before its decay. A similar situation would exist at the $B$ factory for $\Xi^{-}$and $\Omega^{-}$, but with very high statistics. There should thus be no problem observing and studying the $\Omega_{c}^{0}$. 
Table 9.2

$1 / 2^{+}$Charmed Baryons

\begin{tabular}{|c|cc|cc|}
\hline & \multicolumn{2}{|c|}{ CLEO } & \multicolumn{2}{c|}{$B$ Factory } \\
& $430 \mathrm{pb}^{-1}$ & \multicolumn{2}{|c|}{$30 \mathrm{fb}^{-1}$} \\
\hline Decay Mode & $\mathrm{S}$ & $\mathrm{S} / \mathrm{B}$ & $\mathrm{S}$ & $\mathrm{S} / \mathrm{B}$ \\
\hline$\Lambda_{c}^{+} \rightarrow p K^{-} \pi^{+}$ & 680 & 0.1 & $\underline{15,000}$ & 1 \\
$\Lambda_{c}^{+} \rightarrow p K_{s}$ & 122 & 0.5 & 9,000 & .5 \\
$\Lambda_{c}^{+} \rightarrow \Lambda \pi$ & 90 & 0.7 & 6,000 & .7 \\
$\Lambda_{c}^{+} \rightarrow \Xi^{-} K^{+} \pi^{+}$ & 33 & 3 & 2,000 & 3 \\
\hline$\Sigma_{c}^{++} \rightarrow \Lambda_{c}^{+} \pi^{+}$ & 54 & 0.6 & 1,500 & $\sim 2$ \\
$\Sigma_{c}^{0} \rightarrow \Lambda_{c}^{+} \pi^{-}$ & 48 & 0.4 & 1,200 & $\sim 2$ \\
$\Sigma_{c}^{+} \rightarrow \Lambda_{c}^{+} \pi^{0}$ & & & & \\
\hline$\Xi_{c}^{0} \rightarrow \Xi^{-} \pi^{+}$ & 18 & 4 & 1,300 & 4 \\
$\Xi_{c}^{+} \rightarrow \Xi^{-} \pi^{+} \pi^{+}$ & 24 & 1.2 & 1,700 & 1.2 \\
$\Xi_{c}^{\prime 0} \rightarrow \Xi_{c}^{+} \pi^{-}, \Xi_{c}^{0} \gamma$ & & & & \\
$\Xi_{c}^{\prime+} \rightarrow \Xi_{c}^{0} \pi^{+}, \Xi_{c}^{+} \gamma$ & & & & \\
\hline$\Omega_{c}^{0} \rightarrow \Omega^{-} \pi^{+}$ & & & 260 & \\
$\Omega_{c}^{0} \rightarrow \Xi^{-} \bar{K}^{0} \pi^{+}$ & & & 260 & \\
\hline
\end{tabular}

\subsection{Summary of Charm Physics Capabilities}

There are a number of interesting topics in Charm Physics which require larger samples of detected charm. Although the chances for a major discovery that would fundamentally change our picture of the Standard Model are not as high as in bottom physics, there are still many compelling questions to answer. There will be larger charm samples in the next few years, at fixed target machines, CLEO II and BEPC. At the asymmetric $B$ factory, it will be possible to study charm physics with a sensitivity of one order of magnitude beyond these experiments, or two orders of magnitude beyond present experiments.

The recent success of CLEO and ARGUS in charm physics demonstrates the advantages of the $\Upsilon(4 \mathrm{~s})$ as a source of charm. The ability of E691 to study such topics as $D^{0}-\overline{D^{0}}$ mixing and 
semileptonic decays with unprecedented sensitivity is due to the power of precise vertex information. The combination of these advantages at a $B$ factory, along with charm samples of $6 \times 10^{7}$ events with a run at $\mathcal{L}=3 \times 10^{33} \mathrm{~cm}^{-2} \mathrm{sec}^{-1}$, will make it possible to study charm physics as well as at any facility available in the mid-1990's.

Bigi has given a theorist's view of the requirements for studying the most interesting questions in charm physics ${ }^{3}$ "From these goals one derives benchmark figures for New Initiatives in charm decay: one has to be able to

- probe $D^{0}-\overline{D^{0}}$ mixing down to a level of $\sim 10^{-4}$ in $\mathbf{r}_{D}$, and

- search for CP asymmetries down to the $1 \%$ level."

We have shown that one can reach both of these goals with a machine of luminosity $3 \times 10^{33} \mathrm{~cm}^{-2} \mathrm{sec}^{-1}$. These projections are based on Monte Carlo studies, but also agree with straightforward extrapolation of the existing results from CLEO, ARGUS, and E691.

The advantage of the $B$ factory for other charm decay studies is equally dramatic. Typically the observed rates are a factor of 200 or so greater than the best current samples, and the backgrounds are smaller. In addition the full spectroscopy of charmed mesons and baryons will be explored at this machine to a degree that cannot be matched at any other machine. 


\section{REFERENCES}

1. For a review of the status of charm physics see: R.J. Morrison and M.S. Witherell, Ann. Rev. of Nucl. Sci. 39, 183 (1989); D.G. Hitlin, Proceedings of the 1988 Banff Summer Institute, 607 (1989).

2. G. Gladding, review talk at the Third International Symposium on the Decays and Spectroscopy of Heavy Flavors (Ithaca, New York, 1989); and P. Karchin, review talk at the International Symposium on Lepton and Photon Interactions at High Energies (Stanford, California, 1989).

3. I.I. Bigi, UND-IIEP-89-BIG01 (1989).

4. L. Wolfenstein, Phys. Lett. 164 B, 170 (1985); and J.F. Donoghue et al., Phys. Rev. D33, 179 (1986).

5. J.C. Anjos et al., Phys. Rev. Lett. 56, 1239 (1988).

6. H. Albrecht et al., Phys. Lett. B199, 449 (1987).

7. M. Golden and B. Grinstein, preprint FERMILABPUB-89/48-T (1989).

8. F.J. Gilman and R.L. Singleton, SLAC-PUB-5065 (1989).

9. J.L. Rosner, comments Nucl. Part. Phys., 109 (1986).

10. F.J. Gilman, Charm Physics, ed. by Ming-han Ye and Tao Huang (Gordon and Breach, London, 1988), page 37.

11. A. De Rújula, H. Georgi, and S.L. Glashow, Phys. Rev. D12, 147 (1975).

12. T. Bowcock et al. (CLEO Collaboration), submitted to the International Symposium on Lepton and Photon Interactions at High Energies (Stanford, California, 1989).

13. M.S. Alam et al. (CLEO Collaboration), CLNS 89/921 (1989). 
While making the first measurement of $C P$ violation in the $B$ meson system is the primary objective, the program we envision is far broader, encompassing $b, c$ and $\tau$ physics as well

\section{Summary and Conclusions}

7 his report illustrates the richness of the physics program 1 of a high-luminosity, asymmetric energy storage ring facility running at the $\Upsilon(4 \mathrm{~S})$ and the other $\Upsilon$ resonances. The combination of this novel machine, having a peak luminosity in excess of $10^{33} \mathrm{~cm}^{-2} \mathrm{sec}^{-1}$, and a new high quality detector will provide a wealth of data in the area of bottom, charm and $\tau$ physics. Much of this physics will not bc acccssible at any other facility currently available or planned for the future. We propose constructing the facility at SLAC, allowing us to capitalize on the existing PEP complex, thereby reducing the construction time and the cost. We envision a physics program commencing in late 1995, providing a large, international community of particle physicists with at least ten years of exciting and important physics opportunities.

The primary objective of the physics program is the first measurement of $\mathrm{CP}$ violation in $B$ meson decay and, over time, the detailed study of the mechanism of $\mathrm{CP}$ violation. This in and of itself would justify the program; however the production yields of $b \bar{b}, c \bar{c}$ and $\tau^{+} \tau^{-}$are so large, and the kinematic conditions so favorable, that a first-quality physics program in the areas will also develop. These experiments, taken as a whole, provide a set of probes of the Standard Model, some of them of a "make-or-break" nature, which is unparalled in its depth and breadth.

This report has demonstrated that the high-luminosity asymmetric $B$ factory will yield:

1. Searches and studies of rare $b$ and $c$ decays at a level of sensitivity which far exceeds that available at present facilities

2. significant measurements of both $B \bar{B}$ and $D \bar{D}$ mixing

3. A wide range of measurements pertaining to the CabibboKobayashi- Maskawa matrix elements, in particular several measurements of $V_{u b}$

4. Very large samples of reconstructed $B$ and charm decays, permitting detailed studies of hadron dynamics, meson and baryon spectroscopy and, most importantly, a measurement of $f_{B}$. 
5. A new level of understanding of $\Upsilon$ spectroscopy including access to states hitherto unseen, much improved data for understanding the QCD potential and precise measurements of $\alpha_{s}$

6. A rich menu of $\tau$ physics including a measurement of $M_{\nu_{\tau}}$ at the $2 \mathrm{MeV}$ level, the Cabibbo angle to $\leq 0.5 \%$, studies of the structure of the $\tau-W-\nu_{\tau}$ vertex and $\tau$ lifetime and branching fractions.

Several of these physics topics are unique to an asymmetric $\Upsilon(4 \mathrm{~S})$ collider, while others derive a very large statistical advantage from the asymmetric configuration. For example, $\mathrm{CP}$ violation studies involving $\mathrm{CP}$ eigenstate decays of the $B$ system are not possible at a symmetric $\Upsilon(4 S)$ collider because the CP asymmetries integrate to zero. Improved suppression of backgrounds in mcasurements of decays such as $B^{\circ} \rightarrow J / \psi K_{s}^{\circ}, B^{\circ} \rightarrow \pi^{+} \pi^{-}$or $B^{\circ} \rightarrow \rho^{\circ} \rho^{\circ}$ allow a very substantial reduction in the required luminosity for the asymmetric versus the symmetric configuration. We believe that the asymmetric configuration is an essential ingredient for the design of a high luminosity, $\Upsilon(4 \mathrm{~S}) B$ factory; the motivation has been exhaustively spelled out in this Report. A companion document, A Feasibility Study for a Asymmetric $B$ Factory Based on PEP, provides the basis for a credible design of such a machine. What remains is to move forward with this exciting project.
The ability to make directly interpretable measurements of $C P$-violating asymmetries, i.e., those involving decays to $C P$ eigenstates, is unique to an asymmetric configuration 


\title{
APPENDIX
}

Future Developments: The Consequences of Better

\section{Spatial Resolution in Vertex Reconstruction}

\author{
R. Erbacher and W. Vernon
}

$\mathrm{W}_{\mathrm{t}=\mathrm{at}}^{\mathrm{h}}$

hile conventional vertex detectors are certainly adequate

for the measurement of $\mathrm{CP}$-violating processes, further progress in $B$ physics will entail searches for even rarer decay processes, more exotic effects and further exploration of other consequences of CP violation. This increased sensitivity will require many more events than the first generation of CP violation studies. Some of this increase could come as a consequence of improving our ability to completely reconstruct events. We have therefore undertaken a brief study to establish the scale of sensitivity of event reconstruction success rate to possible future improvements which may occur in vertex detector resolution.

One of the more frustrating parts of $B$ physics is the difficulty in reconstructing events so that the decay chain can be accurately determined. A major part of that problem comes from having to link tracks to decaying particles, often $D$ or $D^{*}$ mesons: the combinatorics can lead to large numbers of potential solutions. Neutral particles such as $\gamma^{\prime}$ 's and $\pi^{\circ}$ 's can cause confusion in interpreting the origin of charged tracks. However, if enough "reliable vertices" are found, then the search for correct links to the neutrals has a much higher chance of success. When the vertices are located with sufficient precision and the original production vertex (beam crossing spot size) is of comparable size, there is an additional constraint in the three-momenta match-ups which should help to confirm the particle/vertex assignments. The key here is finding "reliable vertices."

A large portion of the difficulty in finding the tracks which came from a particular vertex stems from a track from another vertex mistakenly appearing to come from the one being constructed. This can happen when a track appears to be closer to the second vertex than to its own (due to measurement and multiple scattering errors) or when the original vertex 
only has one detected track coming from it (due to either track detection inefficiency or a charged decay into a single charge plus neutrals). We have taken a look at the magnitude of this problem by finding how close charged tracks come to the other vertices in an event, before any measuring errors have been imposed. The vertices which are considered here are constrained to come from the decay of a particle which has traveled between 100 and 1000 microns from its parent vertex. About half of the vertices remain after this cut, and these are the ones with some likelihood of being sufficiently isolated so that they can be linked with their proper tracks. Asymmetric collisions of 8 on $3.5 \mathrm{GeV}$ were used to produce these events.

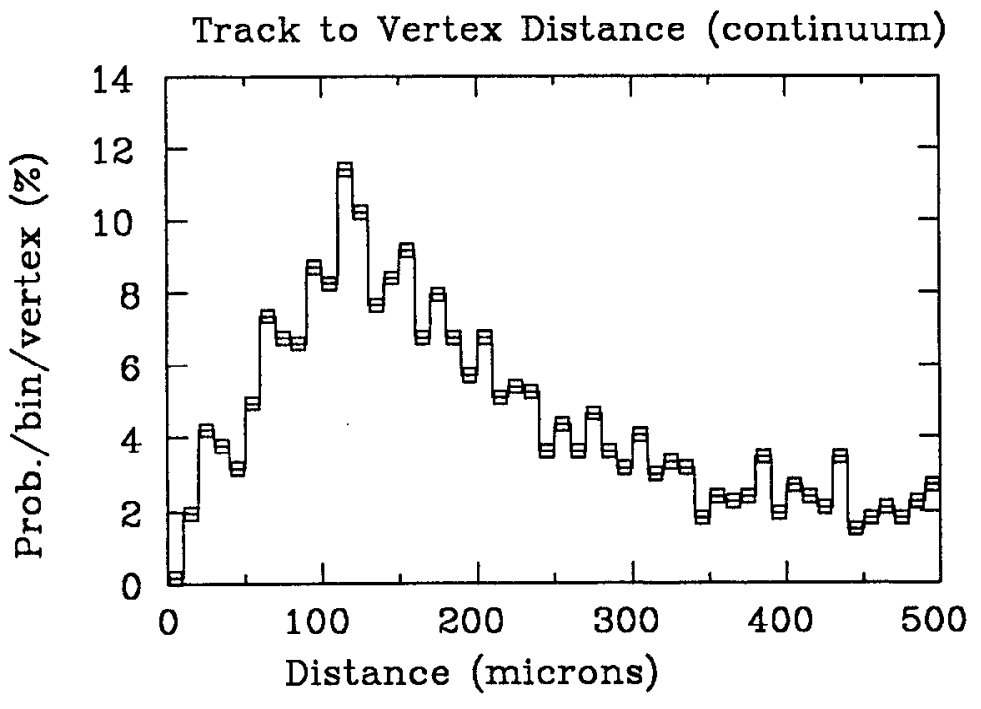

The distribution of distances of closest approach between all of the long-lived charged tracks in an event and the selected vertices is plotted in Figure 1 for the continuum (no $\Upsilon(4 S$ ) ), where most of the closer vertices are $D$ and $D^{*}$ decays. The probability that a track will be within 50 microns of an incorrect vertex is about $10 \%$ per vertex. This is a reasonably clean situation, which indicates that it is possible to do a good job of finding and reconstructing many of the non-Beauty continuum events. Here the smaller number of vertices is the main reason for the low overlap rates, although the large production angle of the $D$ and $D^{*}$ also helps. Yet when the distances for the $\bar{B} B$ events at the $\Upsilon(4 S)$ are plotted for the same vertex selection criterion, the results in Figure 2 show
A crude distance parameter will help to set the scale of the problem

Figure 1. Distribution of distances between all charged tracks and selected vertices for the continuum (no $\Upsilon(4 S)$ ). 
The probability density for tiny distances does not seem to go to zero in the $\bar{B} B$ case

Figure 2. Distribution of distances between all charged tracks and selected vertices for $\bar{B} B$ decays ( $\Upsilon(4 S)$ only). that the overlap rate is now much higher. A vertex resolution of 20 microns would mean a $50 \%$ chance for an incorrect track to link up to a vertex. Or preferably, if one could get down to 10 microns, then the corresponding $25 \%$ probability of incorrect overlap would mean that one might even be able to find one clean vertex per event $90 \%$ of the time, as there are an average of about two of these selected vertices per event.

Track to Vertex Distance (B-4S)

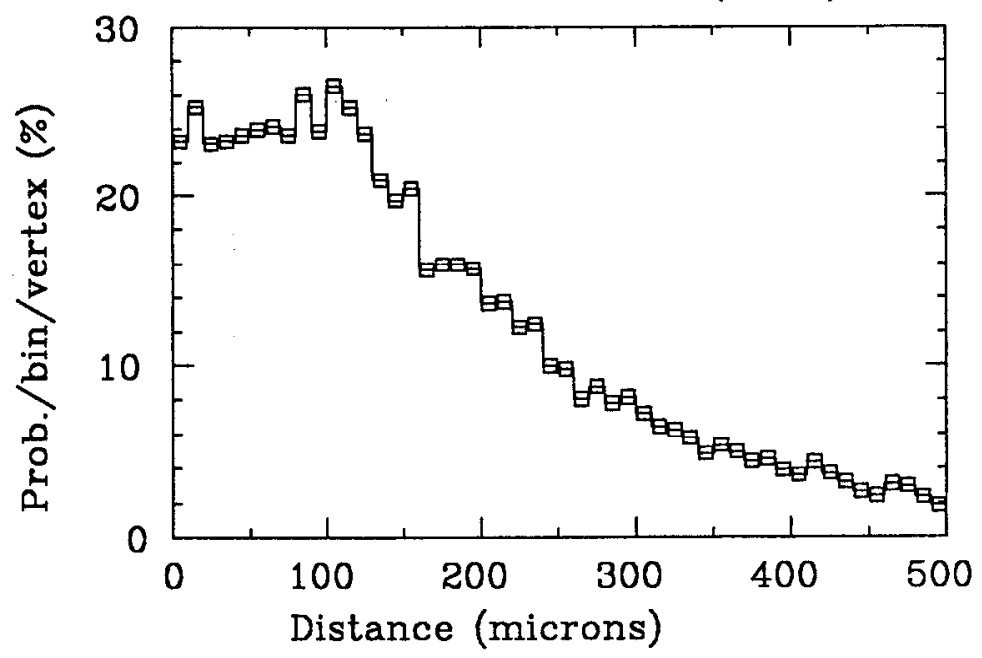

Suppose that we could achieve this mythical 10 micron spatial resolution: what other consequences might follow? Since the $B$ is usually moving along the beam line, typically with an angle of $100 \mathrm{mrad}$, the requirement that the vertex be over 100 microns away and with 10 micron resolution might allow us to measure the $B$ direction with an angular accuracy similar to its production angle. If the original production vertex position were known with comparable precision, at least in the directions transverse to the beam, then the individual $B$-meson directions could be determined with enough accuracy to separate $B$ from $D$ vertices. This will be possible because the $D$ is usually produced with a fairly large angle with respect to the beam line. Also, constrained fitting of the event would improve with this additional spatial information. In Table I we list the spot sizes (radial rms values) which are characteristic of the collision points for the three kinds of machine being proposed for a $\bar{B} B$-Factory. The linac-linac case has a collision size which is similar to that of the SLC, and 
the circular-linac collider operates with a much smaller beam than the circular-circular case, except that the circular-linear machine has never yet been built.

\section{TABLE I}

\begin{tabular}{|c|c|c|}
\hline \multicolumn{2}{|c|}{ SPOT SIZES FOR VARIOUS COLLIDER TYPES } \\
\hline Machine Type & $\sigma_{x}(\mu)$ & $\sigma_{z}(\mathrm{~mm})$ \\
\hline Linear-Linear & 2 & 1 \\
\hline Linear-Circular & 5 & 10 \\
\hline Circular-Circular & 50 & 10 \\
\hline
\end{tabular}

Perhaps it is more relevant to keep track of the other properties of these collider types, especially those features which will have a major impact on the size and composition of the first layer of detector/beam-pipe, so as to insure that the best possible secondary vertex location resolution is achieved. The effectiveness of masking out synchrotron radiation and high energy beam loss particles is related to the dimensions of the beams on their approach to the interaction region; such considerations have a major influence on how near the detector can be to the interaction point. Beam currents, both peak and average, help determine how much electric field shielding and heat removal material will be required in the first layer of the detector and beam pipe wall. We note that in both the linear-linear and circular-linear cases the beam dimensions and the average beam currents are much smaller than in the circular-circular case.

In an optimum environment it ought to be possible to build the first layer of detector out of $0.5 \mu$ gold, $1 \mu$ silver, $1.5 \mu$ copper, $5 \mu$ of aluminum, $0.5 \mathrm{~mm}$ of low density ceramic and $20 \mu$ of silicon pixel detector. The use of a gold, silver and copper multilayer was suggested by Mike Sullivan as part of an $\mathrm{x}$-ray protection scheme which tries to cover a wide range of wavelengths. We would make the ceramic substrate into a low density structure by incorporating gas cooling passages into the layer; the Joule expansion of high pressure helium escaping through small holes cools the layer. Such fabrication
See P. Grosse-Wiesmann, Nucl. Instr. and Meth. A274(1989)21, for the circular-linear story

Smaller beams on the approach to the collision may allow for better suppression of $x$-rays and other junk 
Better vertex resolution leads to better event reconstruction rates which means higher effective luminosity techniques would result in the first layer having an equivalent thickness of $10^{-3}$ radiation lengths and ought to allow vertices to be reconstructed with $10 \mu$ accuracy if the layer is within $1.5 \mathrm{~cm}$ of the origin and the spatial resolution of the pixel is of order $5 \mu$.

What is the physics to be gained from this effort? In each of the major topics discussed in the body of the report there are significant rate limitations associated with inability to reconstruct the event. If the reconstruction efficiency can increase from $1 \%$ to $10 \%$ then it is the same as having increased the luminosity by a factor of ten. Perhaps more significantly, some processes are background dominated and benefit even more from the use of complete reconstruction to suppress background. In a situation where the background could be reduced by a factor of ten, there is an improvement in statistical significance which is the equivalent of having increased luminosity by two orders of magnitude. There are also some processes which benefit directly from the improved vertex resolution; lifetime measurements and mixing studies in the case of $B_{s} \bar{B}_{s}$, for example, are critically dependent on the vertex resolution.

Although the correct study of effects of vertex resolution on reconstruction efficiency will require another year of diligent effort, we feel that the crude estimates used in this section already show that there is considerable sensitivity to the final level of measurement resolution achieved, at least in the case of going from 30 to 10 microns. It is also fairly obvious that the properties of the collider play a major role in this effort to get down to small distances. Finally, it seems very likely that a B-Factory which can achieve high luminosity with modest vertex resolution capability will then be upgraded to an even more productive environment; new beam-pipe/detector technology combined with machine improvements may lead to an order of magnitude improvement in rates for some interesting processes. 